\title{
Atomic data for the Gaia-ESO Survey ${ }^{\star}$
}

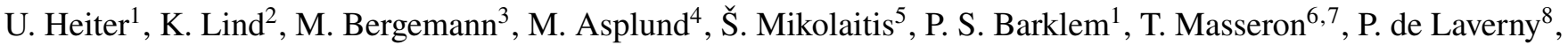 \\ L. Magrini ${ }^{9}$, B. Edvardsson ${ }^{1}$, H. Jönsson ${ }^{10,11}$, J. C. Pickering ${ }^{12}$, N. Ryde ${ }^{11}$, A. Bayo Arán ${ }^{13,14}$, T. Bensby ${ }^{11}$, \\ A. R. Casey ${ }^{15,16,17}$, S. Feltzing ${ }^{11}$, P. Jofré ${ }^{18}$, A. J. Korn ${ }^{1}$, E. Pancino ${ }^{9,19}$, F. Damiani ${ }^{20}$, A. Lanzafame ${ }^{21}$, C. Lardo ${ }^{22}$, \\ L. Monaco ${ }^{23}$, L. Morbidelli ${ }^{9}$, R. Smiljanic ${ }^{24}$, C. Worley ${ }^{15}$, S. Zaggia ${ }^{25}$, S. Randich ${ }^{9}$, and G. F. Gilmore ${ }^{15}$ \\ (Affiliations can be found after the references)
}

Received 11 July 2019 / Accepted 14 October 2020

\begin{abstract}
Context. We describe the atomic and molecular data that were used for the abundance analyses of FGK-type stars carried out within the Gaia-ESO Public Spectroscopic Survey in the years 2012 to 2019. The Gaia-ESO Survey is one among several current and future stellar spectroscopic surveys producing abundances for Milky-Way stars on an industrial scale.

Aims. We present an unprecedented effort to create a homogeneous common line list, which was used by several abundance analysis groups using different radiative transfer codes to calculate synthetic spectra and equivalent widths. The atomic data are accompanied by quality indicators and detailed references to the sources. The atomic and molecular data are made publicly available at the CDS. Methods. In general, experimental transition probabilities were preferred but theoretical values were also used. Astrophysical $g f$-values were avoided due to the model-dependence of such a procedure. For elements whose lines are significantly affected by a hyperfine structure or isotopic splitting, a concerted effort has been made to collate the necessary data for the individual line components. Synthetic stellar spectra calculated for the Sun and Arcturus were used to assess the blending properties of the lines. We also performed a detailed investigation of available data for line broadening due to collisions with neutral hydrogen atoms.

Results. Among a subset of over 1300 lines of 35 elements in the wavelength ranges from 475 to $685 \mathrm{~nm}$ and from 850 to $895 \mathrm{~nm}$, we identified about 200 lines of 24 species which have accurate $g f$-values and are free of blends in the spectra of the Sun and Arcturus. For the broadening due to collisions with neutral hydrogen, we recommend data based on Anstee-Barklem-O'Mara theory, where possible. We recommend avoiding lines of neutral species for which these are not available. Theoretical broadening data by R.L. Kurucz should be used for Sc II, Ti II, and Y II lines; additionally, for ionised rare-earth species, the Unsöld approximation with an enhancement factor of 1.5 for the line width can be used.

Conclusions. The line list has proven to be a useful tool for abundance determinations based on the spectra obtained within the Gaia-ESO Survey, as well as other spectroscopic projects. Accuracies below 0.2 dex are regularly achieved, where part of the uncertainties are due to differences in the employed analysis methods. Desirable improvements in atomic data were identified for a number of species, most importantly Al I, S I, and Cr II, but also Na I, Si I, Ca II, and Ni I.
\end{abstract}

Key words. atomic data - stars: abundances - stars: late-type - surveys

\section{Introduction}

The Gaia-ESO Public Spectroscopic Survey (GES, Gilmore et al. 2012; Randich et al. 2013) started in 2011 and was completed in 2018. High quality spectra were obtained for about $10^{5}$ stars in the Milky Way, predominantly of F-, G-, and K-type. Spectra were obtained with the FLAMES multi fibre facility using the UVES and GIRAFFE spectrographs at the Very Large Telescope at the Paranal Observatory, Chile. Several wavelength regions were covered, mostly 480-680 and 850-900 $\mathrm{nm}$ at different resolutions $(R=\lambda / \Delta \lambda=47000$ and $\sim 20000)$. As part of the GES, these spectra are analysed to determine radial velocities and the following stellar parameters: effective temperatures, surface gravities, and elemental abundances. To perform this analysis, corresponding atomic and molecular data are needed for the observed spectral regions, as well as to determine which lines are suitable for analysis across the range of spectral types. Within the GES, the spectra are analysed independently by different groups, which has the advantage of providing checks on

\footnotetext{
* The atomic and molecular data are only available at the CDS via an anonymous ftp to cdsarc.u-strasbg. fr (130.79.128.5) or via http://cdsarc.u-strasbg.fr/viz-bin/cat/J/A+A/645/A106
}

the analysis. However, in order to limit the potential sources of differences between the analyses, it was decided to use standard input data as far as possible, in particular a common list of lines with corresponding atomic and molecular data (see Pancino et al. 2017). A common line list is also desirable from the point of view that accurate atomic and molecular data are needed to ensure the best possible results from the survey. The critical compilation of such data is a significant and time-consuming task often requiring specialised knowledge and tracking of recent advances in the field (e.g. Barklem 2016).

The purpose of this paper is to describe the common line list and the process of building it for the GES. The line list is also expected to be useful for other surveys with overlapping spectral regions, and for the analysis of F-, G-, and K-type stars in general. The paper is organised as follows. Section 2 describes the general procedure for critical selection and the assessment of the lines for analysis, and their corresponding data. In Sect. 3, the molecular data are described. In Sect. 4, the line list is discussed in general terms, future data needs are identified, and access to the data is described (Sect. 4.3).

Specific aspects of the line data are described in more detail in Appendices A-H. In Appendices B-D, the sources for the 
fundamental properties of the atomic lines (energies, wavelengths, oscillator strengths, etc.) and the quality assessment for the lines in stellar spectra are discussed element by element. In Appendices E-H, the methods for calculation of data for collisional broadening by neutral hydrogen, which are of particular relevance for these spectral types, are described and discussed.

\section{Data selection and assessment}

\subsection{Preselected line list}

The task of defining a standard line list for stellar parameter determination and abundance analysis was started in May 2012. We asked all of the groups participating in the Gaia-ESO analysis of FGK-type stars to provide us with their 'favourite' line lists. In this way we collected lists of lines contained within the standard UVES-580 and GIRAFFE HR21 settings that the groups found particularly appropriate for spectral analysis of the FGK-type stars among Gaia-ESO targets. We note that the UVES-580 setting covers the wavelength ranges of the GIRAFFE HR10 and HR15N settings that were also employed in the GES for cool stars. This resulted in a unique set of 1341 lines for 35 elements (44 species comprising neutral and singly-ionised atoms), which we refer to as the 'preselected line list'. The total number of lines included for each species can be seen in Table 1. These numbers do not include hyperfinestructure or isotopic components (cf. Sect. 2.5). When those are included the total number of transitions is 2631 . The lines cover the wavelength ranges from 475 to $685 \mathrm{~nm}$ and from 850 to $895 \mathrm{~nm}$. The Ca II NIR triplet line at $849.8 \mathrm{~nm}$ is included as well. The first range is somewhat larger than the nominal wavelength region of the UVES-580 setting (480 to $680 \mathrm{~nm}$ ) in order to account for objects with large radial velocities. The second range corresponds to the GIRAFFE HR21 setting.

There are a few lines with fine structure components (levels with different values of the total angular momentum quantum number $J$ ) that have wavelengths within $\sim 0.01 \AA$ from each other. The individual transitions are thus not resolved in stellar spectra. To simplify for equivalent-width based analysis methods, we merged these transitions and added their $g f$-values. The lines in question are listed in Table A.1.

Our objective was to select the best available atomic data for the preselected lines and to provide critical assessments of the atomic data quality and the blending properties of these lines in selected benchmark stars. The intended primary use of this information was the line selection for a homogeneous abundance analysis within the GES, with the best possible accuracy, but it should be useful for other spectroscopic projects as well. We developed an easy-to-use flagging system in order to summarise and communicate the information about the quality of the transition probabilities and the blending properties. For each of these two aspects each line was assigned one of three possible flags for recommended use: $Y$, for "yes, we recommend to use this line", $N$, for "not recommended", or $U$, for "undecided". The general approach for data compilation and assessment is described in Sects. 2.2 and 2.7, while an in-depth description on an element-by-element basis can be found in Appendix B.

In summary, we highly recommend lines flagged with $Y / Y$ for $g f$-value quality and blending property, while we strongly advise against using lines flagged with $N / N$. Lines with other combinations need to be examined and decided upon on a caseby-case basis. We note that we have been more restrictive with $Y$ flags for elements with more spectral lines, such as Fe, compared to other elements with very few lines, such as $\mathrm{O}$.

\subsection{Data compilation and quality assessment for transition probabilities}

We set out to assemble the best possible transition probabilities for all lines, using all literature at hand. In brief, we generally gave highest priority to laboratory measurements, followed by advanced quantum mechanical calculations such as those provided by the Opacity Project ${ }^{1}$ and the MCHF project ${ }^{2}$. When data were not found in such sources we used the semi-empirical calculations by R.L. Kurucz ${ }^{3}$. Measurements of astrophysical nature have not been derived or included at this point. Transition probabilities derived by fitting synthetic to observed stellar spectra are inherently associated with the specific reference object(s) and models used, and would not be applicable to all targets and analysis groups. An exception was made for a few atomic lines located in the vicinity of the Li I $670.8 \mathrm{~nm}$ line, for which astrophysical $g f$-values were derived (see Appendix B.2).

Transition probabilities are given in the form of $\log g f$, and the flags for their quality (hereafter $g f f l a g$ ) were assigned according to the following general scheme: $Y$ indicates data which are considered highly accurate, or which were the most accurate ones available for the element under consideration at the time of compilation; $U$ indicates data for which the quality is not decided; and $N$ indicates data which are considered to have low accuracy. The assignment of the flags for different elements is described in the respective sub-sections of Appendix B.

The starting point for the list of references was the literature sources used in the series of articles on the elemental composition of the Sun by Scott et al. (2015a,b) and Grevesse et al. (2015). This was complemented by further sources as needed. For the quality assessment we were guided by the uncertainties given by atomic data producers for the life-times, branching fractions, etc. measured in the laboratory. Our intention was to make a homogeneous selection of sources for each element. To this effect, lines with data from one and the same source were assigned the same $g f_{-} f l a g$, with the exception of a few Fe lines (see discussion in Appendix B.16). The number of lines to which the different $g f$ flags were assigned for each species is indicated in Table 1. We emphasise that the only purpose of the flags was to provide a qualitative guideline for usage within the Gaia-ESO Survey. They were used to help decide on the usage of a line in case of doubt. However, for future applications the flags should be carefully re-evaluated and replaced by the user's personal assessment of data quality.

\subsection{Reference spectra for illustration of spectral lines}

In this section and in Appendix B we use both calculated and observed spectra of benchmark stars to illustrate the behaviour of spectral lines associated with atomic properties. Here we give some information on these spectra, including stellar parameters, other assumptions, and references to spectroscopic data.

With the optimised set of transition probabilities we computed synthetic spectra of all preselected lines for the parameters of the Sun and Arcturus at a spectral resolution of $R=\lambda / \Delta \lambda=47000$, which is roughly the resolution of the UVES spectra obtained in the GES. For the Sun we used $\left(T_{\text {eff }}[\mathrm{K}]\right.$, $\left.\log g\left[\mathrm{~cm} \mathrm{~s}^{-2}\right]\right)=(5777,4.44)$, similar to the recommended values of $(5771,4.4380)$ given in Heiter et al. (2015), see also Prša et al. (2016), microturbulence $=1 \mathrm{~km} \mathrm{~s}^{-1}$, rotational broadening

\footnotetext{
http://cdsweb.u-strasbg.fr/topbase/topbase.html

2 Multiconfiguration Hartree-Fock database, http://nlte.nist. gov/MCHF /

3 http: //kurucz.harvard.edu
} 
Table 1. Species included in the preselected line list.

\begin{tabular}{|c|c|c|c|c|c|c|c|c|c|c|c|c|c|c|c|c|}
\hline$Z$ & Species & $N_{\text {tot }}$ & $\mathrm{ABO}_{\text {tot }}$ & $Y / Y$ & $\mathrm{ABO}$ & $Y / U$ & $\mathrm{ABO}$ & $U / Y$ & $\mathrm{ABO}$ & $U / U$ & $\mathrm{ABO}$ & $N / Y$ & $N / U$ & $Y / N$ & $U / N$ & $N / N$ \\
\hline 3 & $\mathrm{Li} I$ & 2 & 2 & 0 & 0 & 2 & 2 & 0 & 0 & 0 & 0 & 0 & 0 & 0 & 0 & 0 \\
\hline 6 & $\mathrm{C}_{\mathrm{I}}$ & 4 & 2 & 1 & 1 & 3 & 1 & 0 & 0 & 0 & 0 & 0 & 0 & 0 & 0 & 0 \\
\hline 8 & O I & 4 & 1 & 0 & 0 & 3 & 1 & 0 & 0 & 0 & 0 & 0 & 0 & 1 & 0 & 0 \\
\hline 11 & $\mathrm{Na} \mathrm{I}$ & 11 & 4 & 2 & 2 & 0 & 0 & 3 & 1 & 1 & 0 & 0 & 0 & 0 & 5 & 0 \\
\hline 12 & $\mathrm{Mg}_{\mathrm{I}}$ & 12 & 5 & 3 & 3 & 1 & 1 & 6 & 1 & 2 & 0 & 0 & 0 & 0 & 0 & 0 \\
\hline 13 & Al I & 5 & 2 & 0 & 0 & 0 & 0 & 1 & 0 & 4 & 2 & 0 & 0 & 0 & 0 & 0 \\
\hline 14 & Si I & 45 & 3 & 3 & 2 & 7 & 1 & 2 & 0 & 2 & 0 & 10 & 11 & 2 & 2 & 6 \\
\hline 14 & Si II & 2 & 0 & 0 & 0 & 2 & 0 & 0 & 0 & 0 & 0 & 0 & 0 & 0 & 0 & 0 \\
\hline 16 & $\mathrm{~S}_{\mathrm{I}}$ & 8 & 1 & 0 & 0 & 0 & 0 & 0 & 0 & 8 & 1 & 0 & 0 & 0 & 0 & 0 \\
\hline 20 & $\mathrm{Ca} I$ & 31 & 26 & 12 & 11 & 8 & 5 & 1 & 1 & 0 & 0 & 0 & 0 & 8 & 2 & 0 \\
\hline 20 & Ca II & 8 & 3 & 2 & 2 & 1 & 1 & 0 & 0 & 4 & 0 & 0 & 0 & 0 & 1 & 0 \\
\hline 21 & Sc I & 7 & 3 & 2 & 2 & 3 & 1 & 0 & 0 & 0 & 0 & 0 & 0 & 2 & 0 & 0 \\
\hline 21 & Sc II & 17 & 0 & 2 & 0 & 4 & 0 & 0 & 0 & 0 & 0 & 4 & 1 & 6 & 0 & 0 \\
\hline 22 & Ti I & 105 & 84 & 23 & 22 & 47 & 40 & 0 & 0 & 0 & 0 & 3 & 3 & 24 & 0 & 5 \\
\hline 22 & Ti II & 23 & 0 & 1 & 0 & 10 & 0 & 0 & 0 & 0 & 0 & 1 & 3 & 7 & 0 & 1 \\
\hline 23 & V I & 49 & 46 & 15 & 15 & 16 & 16 & 0 & 0 & 0 & 0 & 3 & 6 & 5 & 0 & 4 \\
\hline 23 & V II & 3 & 0 & 0 & 0 & 0 & 0 & 0 & 0 & 0 & 0 & 0 & 1 & 0 & 0 & 2 \\
\hline 24 & Cr I & 64 & 52 & 9 & 9 & 15 & 15 & 0 & 0 & 0 & 0 & 9 & 11 & 11 & 0 & 9 \\
\hline 24 & CrII & 24 & 24 & 0 & 0 & 1 & 1 & 0 & 0 & 0 & 0 & 1 & 7 & 5 & 0 & 10 \\
\hline 25 & Mn I & 27 & 12 & 3 & 2 & 15 & 9 & 0 & 0 & 0 & 0 & 3 & 4 & 1 & 0 & 1 \\
\hline 26 & $\mathrm{Fe} I$ & 545 & 442 & 83 & 79 & 120 & 104 & 44 & 43 & 84 & 79 & 33 & 75 & 45 & 34 & 27 \\
\hline 26 & $\mathrm{Fe}$ II & 42 & 42 & 6 & 6 & 7 & 7 & 6 & 6 & 7 & 7 & 0 & 0 & 6 & 10 & 0 \\
\hline 27 & Co I & 34 & 22 & 8 & 8 & 14 & 12 & 0 & 0 & 0 & 0 & 1 & 8 & 2 & 0 & 1 \\
\hline 28 & $\mathrm{Ni} I$ & 99 & 86 & 16 & 14 & 14 & 11 & 1 & 1 & 2 & 2 & 19 & 31 & 3 & 1 & 12 \\
\hline 29 & $\mathrm{Cu}$ I & 6 & 0 & 1 & 0 & 4 & 0 & 0 & 0 & 0 & 0 & 0 & 0 & 1 & 0 & 0 \\
\hline 30 & $\mathrm{Zn} \mathrm{I}$ & 2 & 1 & 0 & 0 & 2 & 1 & 0 & 0 & 0 & 0 & 0 & 0 & 0 & 0 & 0 \\
\hline 38 & Sr I & 10 & 0 & 0 & 0 & 1 & 0 & 0 & 0 & 1 & 0 & 0 & 0 & 4 & 3 & 1 \\
\hline 39 & Y I & 6 & 0 & 0 & 0 & 0 & 0 & 0 & 0 & 0 & 0 & 0 & 2 & 0 & 0 & 4 \\
\hline 39 & Y II & 19 & 0 & 4 & 0 & 7 & 0 & 0 & 0 & 0 & 0 & 0 & 0 & 8 & 0 & 0 \\
\hline 40 & $\mathrm{ZrI}$ & 13 & 3 & 5 & 3 & 8 & 0 & 0 & 0 & 0 & 0 & 0 & 0 & 0 & 0 & 0 \\
\hline 40 & Zr II & 3 & 0 & 0 & 0 & 1 & 0 & 0 & 0 & 0 & 0 & 0 & 0 & 1 & 1 & 0 \\
\hline 41 & $\mathrm{Nb}$ I & 8 & 0 & 0 & 0 & 0 & 0 & 0 & 0 & 0 & 0 & 0 & 0 & 8 & 0 & 0 \\
\hline 42 & Mo I & 6 & 1 & 2 & 1 & 3 & 0 & 0 & 0 & 0 & 0 & 0 & 0 & 1 & 0 & 0 \\
\hline 44 & Ru I & 1 & 0 & 0 & 0 & 1 & 0 & 0 & 0 & 0 & 0 & 0 & 0 & 0 & 0 & 0 \\
\hline 56 & Ba II & 4 & 4 & 1 & 1 & 2 & 2 & 0 & 0 & 0 & 0 & 0 & 0 & 1 & 0 & 0 \\
\hline 57 & La II & 6 & 0 & 0 & 0 & 4 & 0 & 1 & 0 & 0 & 0 & 0 & 0 & 1 & 0 & 0 \\
\hline 58 & Ce II & 12 & 0 & 2 & 0 & 4 & 0 & 0 & 0 & 3 & 0 & 0 & 0 & 2 & 1 & 0 \\
\hline 59 & Pr II & 7 & 0 & 1 & 0 & 1 & 0 & 0 & 0 & 0 & 0 & 0 & 0 & 5 & 0 & 0 \\
\hline 60 & Nd II & 53 & 0 & 0 & 0 & 18 & 0 & 2 & 0 & 8 & 0 & 0 & 0 & 17 & 8 & 0 \\
\hline 62 & Sm II & 5 & 0 & 0 & 0 & 4 & 0 & 0 & 0 & 0 & 0 & 0 & 0 & 1 & 0 & 0 \\
\hline 63 & Eu II & 5 & 0 & 0 & 0 & 4 & 0 & 0 & 0 & 0 & 0 & 0 & 0 & 1 & 0 & 0 \\
\hline 64 & Gd II & 1 & 0 & 0 & 0 & 1 & 0 & 0 & 0 & 0 & 0 & 0 & 0 & 0 & 0 & 0 \\
\hline 66 & Dy II & 1 & 0 & 0 & 0 & 1 & 0 & 0 & 0 & 0 & 0 & 0 & 0 & 0 & 0 & 0 \\
\hline
\end{tabular}

Notes. The two preselected hydrogen lines, $\mathrm{H} \alpha$ and $\mathrm{H} \beta$, were omitted from the table. $N_{\text {tot }}$ is the total number of transitions (hyperfine-structure or isotopic components are not included in the count). $\mathrm{ABO}_{\text {tot }}$ is the total number of transitions with line broadening data from the Anstee-BarklemO'Mara theory (ABO data, see Appendices E-H). The remaining columns give the number of lines with the respective combinations of quality flags ( $g$ _flag/synflag, see Sects. 2.2 and 2.7) and the corresponding subset of lines with ABO data.

with $v \sin i=2 \mathrm{~km} \mathrm{~s}^{-1}$, macroturbulence $=2 \mathrm{~km} \mathrm{~s}^{-1}$, and abundances from Grevesse et al. $(2007)^{4}$. For Arcturus we used $\left(T_{\text {eff }}\right.$ $\left.[\mathrm{K}], \log g\left[\mathrm{~cm} \mathrm{~s}^{-2}\right],[\mathrm{Fe} / \mathrm{H}],[\alpha / \mathrm{Fe}]\right)=(4286,1.6,-0.52 \mathrm{dex}$, +0.24 dex), where $\alpha$-elements are those with even atomic numbers from 8 to 22, from Heiter et al. (2015) and Jofré et al.

4 These are the reference abundances that are used throughout the GES analyses. They are also the abundances which are used in the MARCS model atmosphere grid used by GES. Therefore they are not the most recent set of abundances published for the Sun.
(2014, 2015), microturbulence $=1.71 \mathrm{~km} \mathrm{~s}^{-1}, v \sin i=1 \mathrm{~km} \mathrm{~s}^{-1}$, and macroturbulence $=4.5 \mathrm{~km} \mathrm{~s}^{-1}$.

The synthesis was performed with the radiative transfer code SME (Valenti \& Piskunov 1996; Piskunov \& Valenti 2017) based on interpolated MARCS atmospheric models (Gustafsson et al. 2008), which are the same as those employed in the Gaia-ESO analysis. The observational data for the Sun and Arcturus are the Kitt Peak Fourier Transform Spectrometer (FTS) solar and Arcturus flux atlases (Kurucz et al. 1984; Hinkle et al. 2000), degraded to a spectral resolution of 47000 . 
Table 2. Stellar parameters for selected Gaia FGK benchmark stars from Heiter et al. (2015) and Jofré et al. (2014).

\begin{tabular}{rrrl}
\hline \hline$T_{\text {eff }}(\mathrm{K})$ & $\log g\left(\mathrm{~cm} \mathrm{~s}^{-2}\right)$ & {$[\mathrm{Fe} / \mathrm{H}](\mathrm{dex})$} & Name \\
\hline 4374 & 4.63 & -0.33 & 61 Cyg A \\
4474 & 2.51 & 0.25 & $\mu$ Leo \\
4496 & 2.09 & -0.33 & HD 107328 \\
4587 & 1.61 & -2.64 & HD 122563 \\
4858 & 2.90 & 0.13 & $\beta$ Gem \\
4954 & 3.76 & 0.06 & $\delta$ Eri \\
4983 & 2.77 & 0.15 & $\epsilon$ Vir \\
5076 & 4.61 & -0.09 & $\epsilon$ Eri \\
5810 & 4.44 & 0.03 & 18 Sco \\
5868 & 4.27 & -0.86 & HD 22879 \\
6083 & 4.10 & 0.24 & $\beta$ Vir \\
6356 & 4.06 & -2.03 & HD 84937 \\
6554 & 4.00 & 0.01 & Procyon \\
6635 & 4.20 & -0.41 & HD 49933 \\
\hline
\end{tabular}

Figure 1 shows typical example line profiles for four largely unblended $\mathrm{Fe}$ I lines with different $g f_{-}$flag assignments. See Appendix B.16 for details on the Fe I data. The figure illustrates the indicative and statistical nature of the flags. The observed and synthetic spectra agree for both stars in the case of the line with the $Y$ flag (top row in Fig. 1). For $g f_{-} f l a g=U$ or $N$ the synthetic profiles often deviate from the observed ones to different degrees. For the unblended $\mathrm{Fe}$ I lines this is the case for about $40 \%$ of the $g f_{-}$flag $=U$ (e.g. second row in Fig. 1) and $60 \%$ of the $g f_{-}$flag $=N$ lines (e.g. bottom row in Fig. 1), while the remaining lines provide a good fit between observed and synthetic spectra (e.g. third row in Fig. 1). Examples for some of the other elements are given in Appendix B. We point out that the $g f_{-}$flag assignments were based purely on the type of the $g f$-value sources. The performance of the lines in syntheses of the Sun and Arcturus were not taken into account. These are described here for illustrative purposes only.

In addition we use observations of a subset of the Gaia FGK benchmark stars (Heiter et al. 2015; Jofré et al. 2014), including the solar twin 18 Sco and the Arcturus-like star HD 107328. Their spectra were taken from the library of Blanco-Cuaresma et al. $(2014)^{5}$. They were normalised to the continuum and convolved to $R=47000$. The stellar parameters are given in Table 2.

To illustrate the effect on abundance determination when using the quality flags for line selection we computed line abundances for the Sun and three other benchmark stars (Arcturus, the metal-poor dwarf star HD 22879, and 61 Cyg A). This was done for four elements which have a sufficient number of lines for a statistical analysis. Equivalent widths were measured with DAOSPEC (Stetson \& Pancino 2008, 2010) from the spectra used for calibration within the GES, at a spectral resolution of $R=47000$. Abundances were determined from these using the MOOG code (Sneden 1973). The results for the species Si I, CrI, Fe I, and Ni I are presented and discussed in the respective subsections in Appendix B. The observed spreads in line abundances generally support the quality assessment for $g f$-values, although the statistical significance is low for most of these elements.

\footnotetext{
5 http://www.blancocuaresma.com/s/benchmarkstars/, version 2016-05-29.
}

\subsection{Background line list}

Even though the work on the Gaia-ESO line list is focused on the preselected lines, these data are not sufficient for a thorough analysis. We need complete information, as far as possible, on all transitions visible in the observed wavelength ranges in the stars of interest. These data allow us to identify blends for the preselected lines, to include those blends in synthetic spectrum calculations, and to evaluate the quality of spectrum processing (e.g. continuum normalisation). Therefore, the preselected lines were complemented with data for additional atomic lines extracted from the VALD database ${ }^{6}$ (Piskunov et al. 1995; Ryabchikova et al. 2015), as well as data for 27 molecular species (see Sect. 3).

The VALD extraction was done on 2 Sep 2014 using version 820 of the VALD3 database and software, and the default configuration, slightly modified to exclude molecular data and to use line lists without isotopic splitting. The number of lines was limited to those relevant for the GES by using the "Extract Stellar" mode for stellar parameters encompassing those of the target stars. We used a metallicity of $+0.5 \mathrm{dex}$, a microturbulence of $2 \mathrm{~km} \mathrm{~s}^{-1}$, and two combinations of $T_{\mathrm{eff}}$ and $\log g: 6500 \mathrm{~K}$ and 4.0, and $4000 \mathrm{~K}$ and 1.0, respectively. Filtering by a minimum estimated central line depth of 0.001 (without applying any instrumental or rotational broadening) and removing duplicates between the two $T_{\text {eff }}-\log g$ extractions resulted in a total number of about 71000 and 8000 atomic lines contained in the UVES580 and GIRAFFE HR21 wavelength ranges, respectively. The atomic part of the background line list corresponding to these wavelength ranges ${ }^{7}$ is provided together with the preselected line list at the CDS (see Sect. 4.3).

Figures 2 and 3 show the observed and calculated spectra for the Sun and Arcturus for an interval of $8 \mathrm{~nm}$ in the optical region, using the bulk line list (i.e. including preselected and background lines) and the parameters and method described in Sect. 2.3. Most of the observed features are reproduced by the calculations, but we caution that deviations occur in several places. Calculated lines may be too weak or completely missing (e.g. at $534.35 \mathrm{~nm}$ ) or may be too strong compared to the observed lines (e.g. at $538.25 \mathrm{~nm}$ ). This indicates incorrect or lacking atomic data. We would like to point out that we do not provide quality flags for the lines in the background line list (except for some of the Fe I lines, see Appendix B.16). However, the VALD extraction followed the quality ranking of the sources in the database recommended by the VALD team.

\subsection{Hyperfine structure components and isotopic splitting}

In the case of species with non-zero nuclear spin $I^{8}$ the interaction between the nucleus and the electrons may cause a splitting of the fine structure levels into several hyperfine levels. The corresponding hyperfine transitions can be seen in high-resolution spectra as several hyperfine structure (HFS) components for individual atomic lines. Even when the HFS components are not resolved, as is the case for the Gaia-ESO spectra, they must be taken into account in the abundance analysis. In this case, HFS can be regarded as an additional broadening mechanism, altering both the shape of the line profile and the total line intensity.

\footnotetext{
6 http://vald.astro.uu.se

7 From 4750 to $6850 \AA$ and from 8488 to $8950 \AA$.

8 That is, all isotopes with odd baryon number, or with even baryon number and odd proton number, ${ }^{6} \mathrm{Li}$ being the only one representing the latter case among the stable isotopes with non-negligible Solar System abundances.
} 

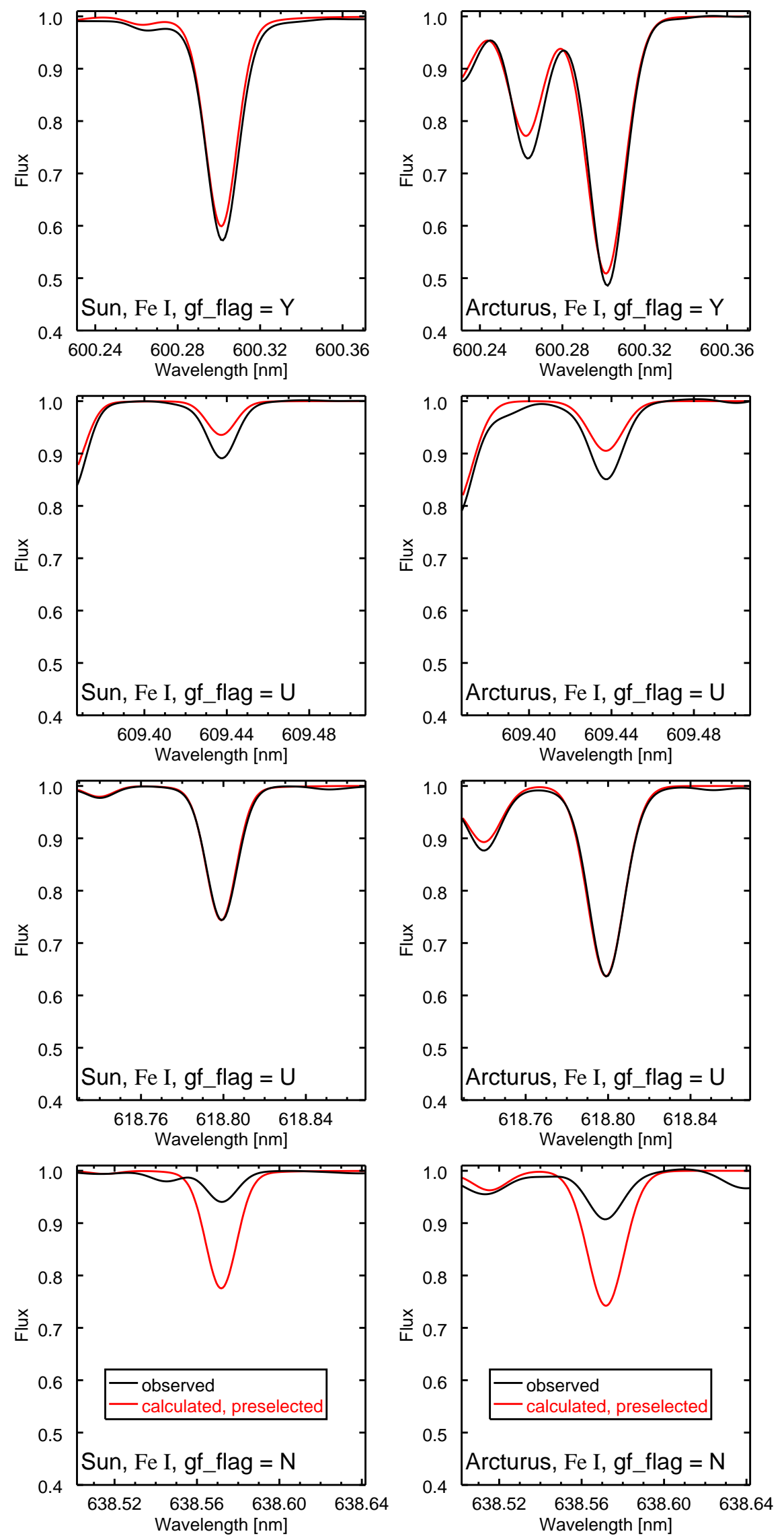

Fig. 1. Comparison of observed and calculated line profiles around four of the preselected Fe I lines with different $g f \_$flag assignments for the Sun (left) and Arcturus (right). Black lines: observations, red lines: calculations including preselected spectral lines only. All of these lines are flagged with $Y$ with respect to their blending properties. We would like to point out that the $g f$ flag assignments were based purely on the type of the $g f$-value sources. The performance of the lines in syntheses of the Sun and Arcturus were not taken into account. These are shown here for illustrative purposes only. 

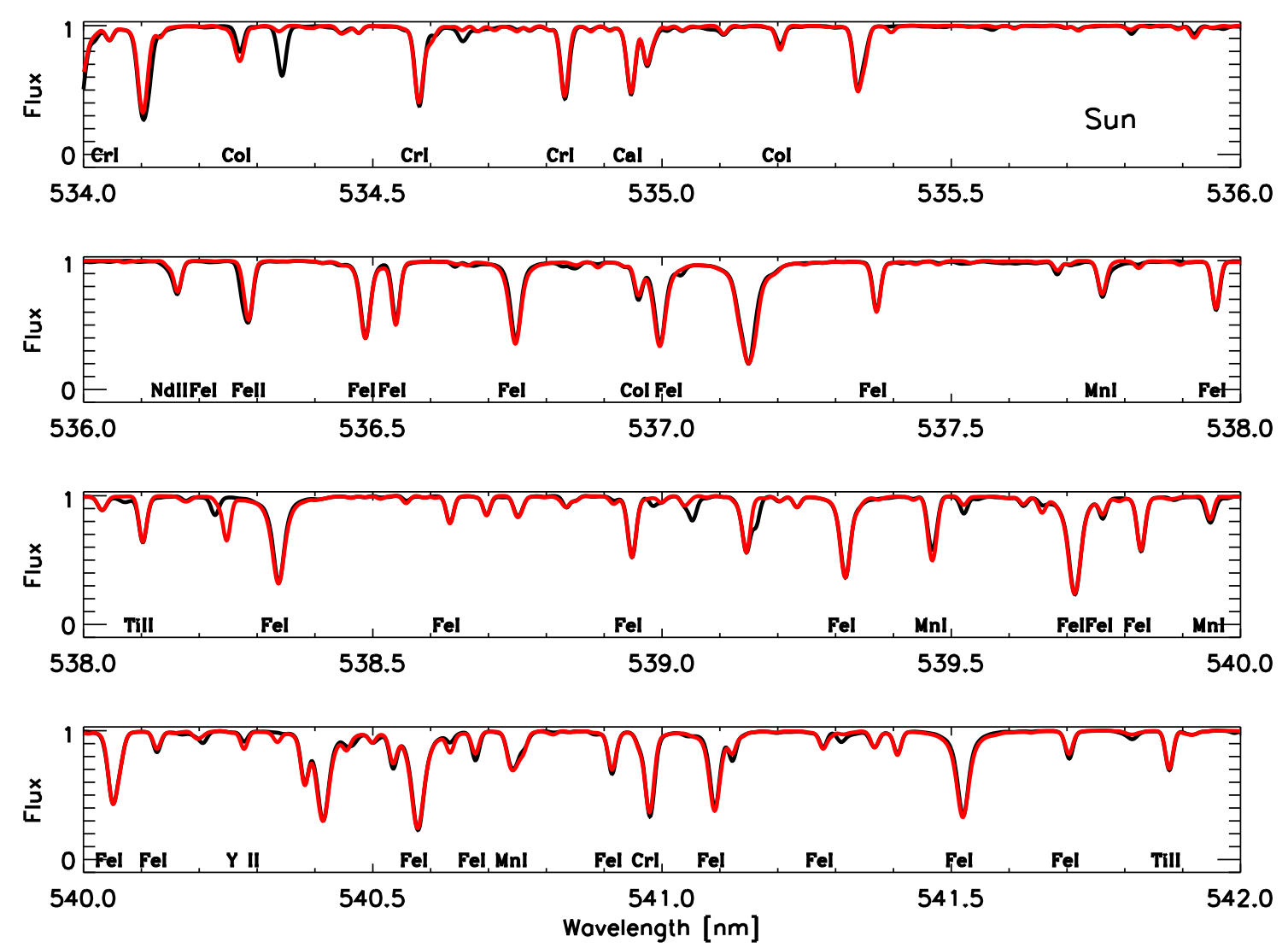

Fig. 2. Observed (black) and calculated (red) spectra for the Sun for an $8 \mathrm{~nm}$-wide interval in the optical region. The Gaia-ESO bulk line list was used as input for the calculations, which includes preselected and background lines. Some of the strongest preselected lines are labelled by their species.
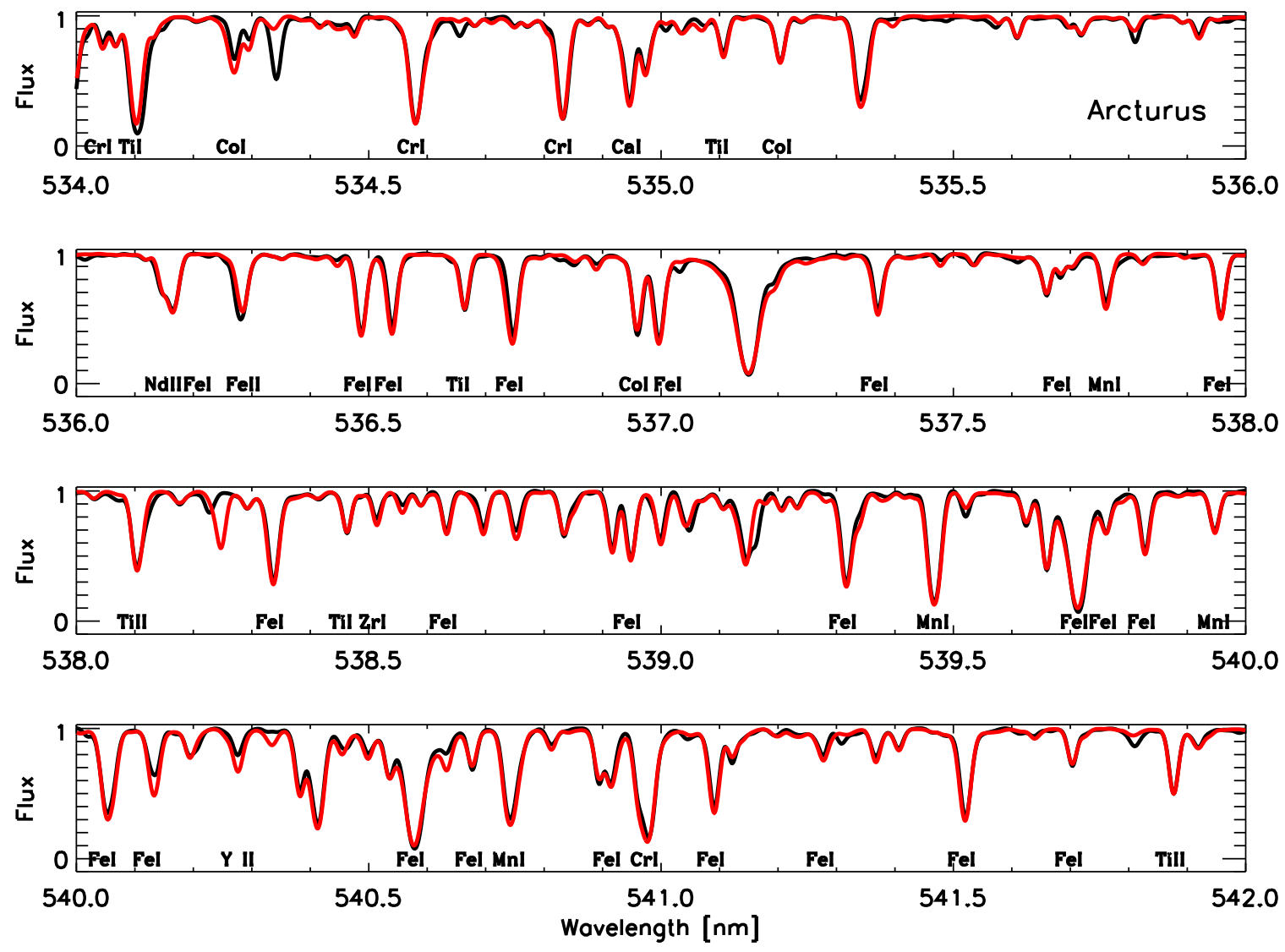

Fig. 3. Same as Fig. 2, but for Arcturus.

A106, page 6 of 63 
Table 3. Isotope information for the elements included in the preselected line list.

\begin{tabular}{|c|c|c|c|c|c|c|c|}
\hline $\begin{array}{r}Z \\
(1) \\
\end{array}$ & $\begin{array}{l}\text { El. } \\
(2)\end{array}$ & $\begin{array}{c}\mathrm{I} \\
(3) \\
\end{array}$ & $\begin{array}{l}\mathrm{H} \\
(4) \\
\end{array}$ & $\begin{array}{l}\text { Baryon numbers } \\
(5)\end{array}$ & $\begin{array}{l}\text { Rel. abund. (\%) } \\
(6)\end{array}$ & $\begin{array}{l}\text { Reference } \\
(7)\end{array}$ & $\begin{array}{l}\text { Nucl. spin } \\
(8)\end{array}$ \\
\hline 3 & $\mathrm{Li}$ & $\mathrm{y}$ & $\mathrm{n}$ & $6: 7$ & 7.6:92.4 & Qi et al. (1997) & $1,1.5$ \\
\hline 6 & $\mathrm{C}$ & $\mathrm{n}$ & $\mathrm{n}$ & $12: 13$ & 99:1 & Chang \& Li (1990) & 0.5 \\
\hline 8 & $\mathrm{O}$ & & & 16 & 99.8 & Baertschi (1976); Li et al. (1988) & \\
\hline 11 & $\mathrm{Na}$ & & $\mathrm{n}$ & 23 & 100 & White et al. (1956) & 1.5 \\
\hline 12 & $\mathrm{Mg}$ & $\mathrm{n}$ & $\mathrm{n}$ & $24: 25: 26$ & $79: 10: 11$ & Bizzarro et al. (2011) & 2.5 \\
\hline 13 & $\mathrm{Al}$ & & $\mathrm{n}$ & 27 & 100 & White et al. (1956) & 2.5 \\
\hline 14 & $\mathrm{Si}$ & $\mathrm{n}$ & $\mathrm{n}$ & $28: 29: 30$ & $92: 5: 3$ & Gonfiantini et al. (1997) & 0.5 \\
\hline 16 & $\mathrm{~S}$ & $\mathrm{n}$ & $\mathrm{n}$ & $32: 33: 34$ & $95: 1: 4$ & Ding et al. (2001) & 1.5 \\
\hline 20 & $\mathrm{Ca}$ & $\mathrm{n}$ & & $40: 42: 44$ & $97: 1: 2$ & Moore \& Machlan (1972) & \\
\hline 21 & $\mathrm{Sc}$ & & $\mathrm{y}$ & 45 & 100 & Leland (1950) & 3.5 \\
\hline 22 & $\mathrm{Ti}$ & $\mathrm{n}$ & $\mathrm{n}$ & $46: 47: 48: 49: 50$ & $8: 7: 74: 5: 5$ & Shima \& Torigoye (1993) & $2.5,3.5$ \\
\hline 23 & V & & $\mathrm{y}$ & 51 & 99.8 & Flesch et al. (1966) & 3.5 \\
\hline 24 & $\mathrm{Cr}$ & $\mathrm{n}$ & $\mathrm{n}$ & $50: 52: 53: 54$ & $4: 84: 10: 2$ & Shields et al. (1966) & 1.5 \\
\hline 25 & $\mathrm{Mn}$ & & $\mathrm{y}$ & 55 & 100 & Leipziger (1963) & 2.5 \\
\hline 26 & $\mathrm{Fe}$ & $\mathrm{n}$ & $\mathrm{n}$ & $54: 56: 57$ & 6:92:2 & Taylor et al. (1992) & 0.5 \\
\hline 27 & Co & & $\mathrm{y}$ & 59 & 100 & Leipziger (1963) & 3.5 \\
\hline 28 & $\mathrm{Ni}$ & $\mathrm{n}$ & $\mathrm{n}$ & $58: 60: 61: 62: 64$ & $68: 26: 1: 4: 1$ & Gramlich et al. (1989) & 1.5 \\
\hline 29 & $\mathrm{Cu}$ & $\mathrm{y}$ & $\mathrm{y}$ & $63: 65$ & $69: 31$ & Shields et al. (1965) & $1.5,1.5$ \\
\hline 30 & $\mathrm{Zn}$ & $\mathrm{n}$ & $\mathrm{n}$ & $64: 66: 67: 68: 70$ & $49: 28: 4: 18: 1$ & Ponzevera et al. (2006) & 2.5 \\
\hline 38 & $\mathrm{Sr}$ & $\mathrm{n}$ & $\mathrm{n}$ & $86: 87: 88$ & $10: 7: 83$ & Moore et al. (1982) & 4.5 \\
\hline 39 & $\mathrm{Y}$ & & $\mathrm{n}$ & 89 & 100 & Collins et al. (1957) & 0.5 \\
\hline 40 & $\mathrm{Zr}$ & $\mathrm{n}$ & $\mathrm{n}$ & 90:91:92:94:96 & $51: 11: 17: 17: 3$ & Nomura et al. (1983) & 2.5 \\
\hline 41 & $\mathrm{Nb}$ & & $\mathrm{n}$ & 93 & 100 & White et al. (1956) & 4.5 \\
\hline 42 & Mo & $\mathrm{n}$ & $\mathrm{n}$ & 92:94:95:96:97:98:100 & 15:9:16:17:10:24:10 & Mayer \& Wieser (2014) & $2.5,2.5$ \\
\hline 44 & $\mathrm{Ru}$ & $\mathrm{n}$ & $\mathrm{n}$ & 96:98:99:100:101:102:104 & $5: 2: 13: 13: 17: 31: 19$ & Huang \& Masuda (1997) & $2.5,2.5$ \\
\hline 56 & $\mathrm{Ba}$ & $\mathrm{y}$ & $\mathrm{y}$ & $134: 135: 136: 137: 138$ & $2: 7: 8: 11: 72$ & Eugster et al. (1969) & $1.5,1.5$ \\
\hline 57 & $\mathrm{La}$ & & $\mathrm{y}$ & 139 & 100 & de Laeter \& Bukilic (2005) & 3.5 \\
\hline 58 & $\mathrm{Ce}$ & $\mathrm{n}$ & & 140:142 & $88: 11$ & Chang et al. (1995) & \\
\hline 59 & $\operatorname{Pr}$ & & $\mathrm{y}$ & 141 & 100 & Collins et al. (1957) & 2.5 \\
\hline 60 & $\mathrm{Nd}$ & $\mathrm{y}$ & $\mathrm{y}$ & $142: 143: 144: 145: 146: 148: 150$ & $27: 12: 24: 8: 17: 6: 6$ & Zhao et al. (2005) & $3.5,3.5$ \\
\hline 62 & $\mathrm{Sm}$ & $\mathrm{n}$ & $\mathrm{y}$ & $144: 147: 148: 149: 150: 152: 154$ & $3: 15: 11: 14: 7: 27: 23$ & Chang et al. (2002) & $3.5,3.5$ \\
\hline 63 & $\mathrm{Eu}$ & $\mathrm{n}$ & $\mathrm{y}$ & $151: 153$ & $48: 52$ & Chang et al. (1994) & $2.5,2.5$ \\
\hline 64 & $\mathrm{Gd}$ & $\mathrm{n}$ & $\mathrm{n}$ & $154: 155: 156: 157: 158: 160$ & $2: 15: 20: 16: 25: 22$ & Eugster et al. (1970) & $1.5,1.5$ \\
\hline 66 & Dy & $\mathrm{n}$ & $\mathrm{n}$ & $160: 161: 162: 163: 164$ & $2: 19: 25: 25: 28$ & Chang et al. (2001) & $2.5,2.5$ \\
\hline
\end{tabular}

Notes. Columns 3 and 4 indicate whether isotopic and/or HFS components are included in the line list (y) or not (n). These fields are empty when the effect does not apply. Column 5 gives the baryon numbers of stable isotopes contributing at least $1 \%$ to the abundance in the Solar System. Column 6 gives the corresponding relative isotopic abundances in per cent, with reference in Col. 7. Column 8 gives the nuclear spins of the isotopes with odd baryon numbers (except for $\mathrm{Li}$ ).

Table 3 lists isotope information for the elements included in the preselected line list.

The HFS part of the Gaia-ESO line list was constructed in the following way. The difference in energy of the hyperfine levels from the fine structure level with a given total electronic angular momentum quantum number $J$ was calculated with the Casimir equation (Casimir 1936, cf. Kopfermann 1958 and Eq. (1) in Pickering 1996). The energy difference depends only on the quantum numbers $J, I$, and $F$, where the latter is associated with the total angular momentum of the atom. The equation consists of two terms corresponding to the magnetic dipole and the electric quadrupole interactions between electron and nucleus. The respective contributions of these interactions are parametrised by the HFS constants $A$ and $B$, which can be empirically determined for any given fine structure level. The number of components for a particular species and transition are governed by selection rules for the $F$ values of the levels involved. The relative intensities of the HFS components were calculated from the line strength formulae derived in the 1920 s for fine-structure multiplets in the Russel-Saunders (LS) coupling scheme (e.g. Eq. (2) in Chapter IX.2 in Condon \& Shortley 1935). To use these formulae for HFS the electron spin quantum number $S$ is replaced by $I$, the orbital angular momentum quantum number $L$ by $J$, and $J$ by $F^{9}$.

For the current work HFS splittings were taken into account for the lower and upper levels of the transitions included in the preselected line list for those elements for which an impact on abundance analysis is expected, whenever laboratory data for the $A$ and $B$ constants were available. In the cases where the available HFS data were incomplete ( $A$ and $B$ constants available only for one of the two levels), the missing $A$ and $B$ constants were set to zero in the computation of the HFS components (12 V I lines, three $\mathrm{Cu}$ I lines, $30 \mathrm{Nd}$ II lines, one $\mathrm{Sm}$ II line). For each transition the HFS components were co-added within bins of $0.01 \AA$.

9 The resulting equations are given for example in Appendix B in Prochaska et al. (2000), with an obvious typo in their Eq. (B9), where $L$ should be replaced by $F$. 
Detailed comments on the selection of $A$ and $B$ values for Sc I, V I, Mn I, Co I, Cu I, Ba II, La II, Pr II, Nd II, Sm II, and Eu II, as well as data tables can be found in Appendices B and C.

Isotopic splitting. For species with several stable isotopes of non-negligible abundances we provide transition data for each isotope separately, where available. For a given electronic state the different atomic masses of the isotopes result in different energy levels. Thus, a given transition can be regarded as split into several lines with different wavelengths for different isotopes. We would like to point out that the transition probabilities given for each isotopic component are the same. Accordingly, line list users need to scale the $g f$-values for isotopes by their relative abundances in the Solar System (Meija et al. 2016) for 'normal' stars, or as applicable for other isotopic compositions. The data used to calculate the isotopic splitting (IS) for the transitions under investigation are described and tabulated in Appendices B and C.

\subsection{Other atomic data}

So far we have discussed the atomic data needed to model the strengths of radiative transitions of the neutral and singly ionised atoms dominating the photospheres of FGK stars. However, to solve the radiative transfer problem and produce a synthetic spectrum a wealth of additional data are needed. These include data to describe the intrinsic widths and shapes of spectral lines (damping profile parameters), ionisation energies and partition functions to determine level populations under the assumption of local thermodynamic equilibrium, and continuous opacities. Most of these are provided as part of the Gaia-ESO line list together with the transition probabilities, and for others we refer to recent publications.

Natural or radiative broadening is caused by the limited lifetimes of the atomic states involved in the transitions. The width of the resulting damping profile is given by the sum of all transition rates for spontaneous deexcitations of both the upper level and the lower level. The radiative damping widths provided by R.L. Kurucz as part of his atomic structure calculations ${ }^{10}$ were included in the Gaia-ESO line list (via the VALD database). These data are available for many of the preselected lines. The exceptions are all of the Al I, Zn I, and Sr I lines, some of the $\mathrm{Na}$ I, Mg I, and Y II lines, and all lines for elements with $Z>40$. For the lines without calculated radiative damping widths one can resort to using the classical description of a spectral line as a damped harmonic oscillator in a two-level atom ${ }^{11}$.

Further broadening of spectral lines is caused by elastic collisions between particles in the stellar atmosphere. Collisional broadening of hydrogen lines is addressed in Appendix B.1. Broadening of metal lines via the quadratic Stark effect due to impacting electrons and ions results in a damping profile which is parametrised by the Stark damping parameter. This effect is in general not very important in the atmospheres of FGKtype stars, as they contain few charged particles compared to the number of neutral particles (for exceptions see discussion in Barklem 2016, Sect. 4.1.2). For completeness, Stark broadening data were extracted from the VALD database and included in the Gaia-ESO line list, where available. In the same way as

10 http://kurucz . harvard . edu/atoms . html

11 Radiative damping width $\gamma_{\mathrm{rad}}=8 \pi^{2} e^{2} 10^{-7} c /\left(3 m_{\mathrm{e}} \lambda^{2}\right)$, at wavelength $\lambda$, where all quantities are in SI units. It is not recommended to use this approximation for lines where calculated $\gamma_{\mathrm{rad}}$ values are available. The approximate value can be up to 1 dex smaller or larger than the calculated value. For example, for the Ca II NIR triplet lines, $\log \gamma_{\text {rad }}($ calculated $)=8.2$, while $\log \gamma_{\text {rad }}($ approximate $)=7.5$. for radiative broadening, they come as a by-product of the calculations by R.L. Kurucz who computed them from sums over all possible transitions to a given level as described in Kurucz (1981, p. 75).

Collisional broadening by neutral hydrogen atoms is important for many metal lines, and is discussed in detail separately in Appendices E-H. In summary, collisional line widths for neutral and ionised Fe lines computed with the Anstee-Barklem-O'Mara (ABO) theory (Anstee \& O'Mara 1991 and successive expansions by P.S. Barklem and collaborators) were compared to those computed by R.L. Kurucz and with the Unsöld recipe, which are based on Lindholm-Foley theory. The ratios between line widths from different theoretical approaches show a large spread, in particular for high values of the excitation energy. As the ABO theory is considered the most reliable theory (Barklem 2016), new broadening data were calculated according to the ABO theory for 41 lines of Fe I and eight other neutral species. These were included in the Gaia-ESO line list, together with previously available data for all other lines based on the ABO theory or provided by Kurucz, which had been extracted from the VALD database. Based on the analysis in Appendices E-H, we recommend avoiding lines of neutral species for which ABO data are not available (cf. Table 1). For lines of ionised species without $\mathrm{ABO}$ data that have low excitation energies, data by Kurucz should be used where available (Sc II, Ti II, and Y II lines), otherwise the Unsöld approximation with an enhancement factor of 1.5 for the line width can be used (lines of rare-earth species).

For ionisation energies for atoms we refer to the NIST Atomic Spectra Database ${ }^{12}$ (Kramida et al. 2018), or Table 4 in Barklem \& Collet (2016). Barklem \& Collet (2016) calculated partition functions for all elements from $\mathrm{H}$ to $\mathrm{U}$ and the first three ionisation stages, for temperatures up to $10000 \mathrm{~K}$ (their Table 8), based on excitation energies from the NIST ASD. Their data agree very well with those of Irwin (1987) for temperatures in common (i.e. above $1000 \mathrm{~K}$ ) for most species. However, for some of the rare-earth elements, differences of up to $50 \%$ are seen (their Figs. 5 and 6). For La II the new partition functions are lower than Irwin (1987) at low temperatures, for Sm II and Eu II they are higher at low temperatures, and for Pr II and Dy II they are higher at high temperatures.

Finally, calculations of continuous fluxes are needed to be able to compare synthetic spectra to observations normalised to the continuum. This requires a large amount of input data for describing processes that are responsible for continuous opacities. These are bound-free and free-free transitions as well as scattering processes for numerous species which are abundant in cool stellar atmospheres. We did not define a standard set of data to be used for this aspect within GES. Instead, we refer to the data commonly used by the codes employed for Gaia-ESO data analysis. For example, the SME package and the MOOG code compute continuous opacities using adapted versions of the subroutines embedded in the ATLAS9 code by R.L. Kurucz ${ }^{13}$ (Kurucz 1970, p. 73). Obviously, the same routines are used in the SYNTHE code by Kurucz. For radiative transfer codes associated with the MARCS model atmosphere package (e.g. Turbospectrum) references for continuous opacity data are given in Table 1 of Gustafsson et al. (2008) and are discussed in their Sect. 4. For other codes see the references given in Smiljanic et al. (2014), who describe most of the Gaia-ESO analysis methods.

${ }^{12}$ NIST ASD: http://physics.nist.gov/PhysRefData/ASD/ ionEnergy.html

${ }^{13}$ http://kurucz . harvard. edu/programs/atlas9/atlas9. for 


\subsection{Blending properties for the sun and arcturus}

In order to assess the blending properties of the preselected lines, two spectra each were calculated for parameters of the Sun and Arcturus (as described in Sect. 2.3). For one of the spectra we used only the preselected lines as input, and for the other one all the blending atomic and molecular lines from the background line list were included (Sects. 2.4 and 3).

The flags for blending properties (hereafter synflag) were assigned after visual inspection of the three overplotted line profiles (the two synthetic ones and the observed one) according to the following general scheme: $Y$ indicates that the line is unblended or only blended with a line of the same species in both stars; $U$ indicates that the line may be inappropriate in at least one of the stars; $N$ indicates that the line is strongly blended with line(s) of different species in both stars. The number of lines to which the different synflags were assigned for each species is indicated in Table 1.

Figure 4 shows typical example line profiles for four Fe I lines with accurate $g f$-values $\left(g f \_l a g=Y\right)$ and with different synflag assignments. The top row shows the same line at $600.3 \mathrm{~nm}$ as the top row of Fig. 1. It is assessed to be unblended ( ynflag $=Y$ ), because all three spectra lie on top of each other. The second and third rows of Fig. 4 show examples for lines with undecided blending properties (synflag $=U$ ). The line at $522.5 \mathrm{~nm}$ has a weak blend at the red side in both stars and a possible additional unidentified blend as seen from the comparison with the observed Arcturus spectrum. The line at $540.1 \mathrm{~nm}$ is almost blend-free in the Sun but strongly blended in Arcturus. In addition, the data from the background line list around this line are incorrect, as is obvious from the comparison with the observed Arcturus spectrum. The bottom row of Fig. 4 shows an example for a line which is clearly blended in both stars $($ synflag $=N$ ).

We did not consider whether the strength of a line is appropriate for analysis in a specific star (i.e. not too weak or too strong), since this will vary much between survey targets. The blending assessment of a line should thus be valid only in comparison to other lines of the same species and line strength. This means for example that synflag $=Y$ has been assigned to unblended lines in Arcturus even when they were not detectable in the Sun.

Observed line profiles for other benchmark stars with a wider range of stellar parameters are shown for the same four Fe I lines in Fig. 5 (see Sect. 2.3 for information on the spectra). For two stars of similar temperature and gravity (e.g. dark brown dotted lines representing cool giants) the variation in line strength is due to the difference in metallicity. The synflag $=Y$ line seems blendfree in all stars (except the coolest dwarf star $61 \mathrm{Cyg}$ A). The synflag $=U$ and $N$ lines are unblended in the warmer dwarfs and the metal-poor giant HD 122563, but they are blended in giant stars and cooler dwarfs. Further examples of Fe I lines with synflag $=N$ are those at 516.6 and $517.2 \mathrm{~nm}$, which lie on the wings of the $\mathrm{Mg} \mathrm{I}$ b 516.7 and $517.3 \mathrm{~nm}$ lines. The line at $559.5 \mathrm{~nm}$ is a blend with a preselected Ca I line. Several further Fe I lines have weak blends in the Sun but strong blends in Arcturus (e.g. 547.3, $623.1 \mathrm{~nm}$ ), and the reverse case is also encountered (e.g. 625.4, $635.9 \mathrm{~nm}$ ). Examples for other species are given in Appendix B.

\section{Molecular data}

In addition to atomic data, we also include molecular data. In fact, the molecular transitions are much more numerous than the atomic transitions. Thus, it is crucial to include extensive molecular line lists for determining stellar parameters and identifying and fitting atomic line blends, although they also may be used for abundance determination and to derive isotopic ratios. Priority was given to molecules which contribute significantly to the absorption in the spectra of $\mathrm{G}$ or K-type stars. This includes $\mathrm{CH}$, $\mathrm{NH}, \mathrm{OH}, \mathrm{C}_{2}, \mathrm{CN}, \mathrm{MgH}, \mathrm{SiH}, \mathrm{CaH}, \mathrm{FeH}, \mathrm{TiO}, \mathrm{VO}$, and $\mathrm{ZrO}$ (and their isotopologues). Although the best line lists available in the literature were used (Table 4) ${ }^{14}$, the quality of the molecular data varies from one molecule to the other.

For $\mathrm{CH}, \mathrm{NH}, \mathrm{OH}$, and $\mathrm{MgH}$, improved line lists were computed using high-quality laboratory line positions (better than $10^{-3} \AA$ ) combined with an accurate computation of transition moments (used to derive $\log g f$ for molecules). The laboratory line positions were used as input in the programme for simulating molecular spectra PGopher ${ }^{15}$ (Western 2017) in parallel with the programmes RKR1 and LEVEL ${ }^{16}$ (Le Roy 2017a,b) for derivation of the line intensities. Radiative broadening parameters were also computed whenever the information of the full electronic structure of the molecule was available. An illustration of the procedure for the case of $\mathrm{CH}$ can be found in Masseron et al. (2014). The line lists for the remaining molecules from the literature in general make use of the Born-Oppenheimer approximation to determine the positions and assume identical oscillator strengths for all isotopologues. In particular, the line lists for $\mathrm{SiH}$, $\mathrm{CaH}, \mathrm{FeH}, \mathrm{TiO}, \mathrm{VO}$, and $\mathrm{ZrO}$ suffer from larger uncertainties, which for positions can sometimes reach several $\AA$.

The molecular data are available at the CDS for the same wavelength ranges as the atomic data. The content of the CDS table is described in Sect. 4.3.

In addition to line positions and oscillator strengths, dissociation energies (Table 4) and partition functions are required to compute the molecular equilibrium. The partition functions used in this work are given in Table A.2.

Recently, Barklem \& Collet (2016) reviewed partition functions and dissociation energies for a large number of diatomic molecules. Regarding dissociation energies, our selected values agree within $1 \%$ with the selection of Barklem \& Collet (2016), except for $\mathrm{CaH}$. However, because this molecule only appears in the coolest stars, this discrepancy should only have a marginal impact on the Gaia-ESO analysis. Concerning partition functions, an agreement better than $10 \%$ for temperatures lower than $5000 \mathrm{~K}$ is found between our adopted values and the compilation of Barklem \& Collet (2016), except for FeH. We note that this latter molecule contributes only in the HR21 setting of the GIRAFFE instrument and only for very cool stars.

\section{Discussion and outlook}

\subsection{Selected examples for the application of the line list}

The Gaia-ESO line list has been used within the consortium for the determination of atmospheric parameters and abundances of calibration stars, stars across Galactic populations, and stars in several clusters. The results have been presented in over 50 refereed articles. Other spectroscopic surveys have also started to use the Gaia-ESO line list as a basis for their analyses. Examples for these are the EMBLA (Howes et al. 2016), GALAH (De Silva et al. 2015), and OCCASO (Casamiquela et al. 2016) surveys. Here, we briefly mention some of these works.

The Gaia FGK benchmark stars consist of about 30 well-known stars and were the main calibrators in the GES

14 For further and more recent references for data for these molecules see, e.g., Tennyson et al. (2020).

15 http://pgopher.chm.bris.ac.uk

16 http://leroy.uwaterloo.ca/programs.html 

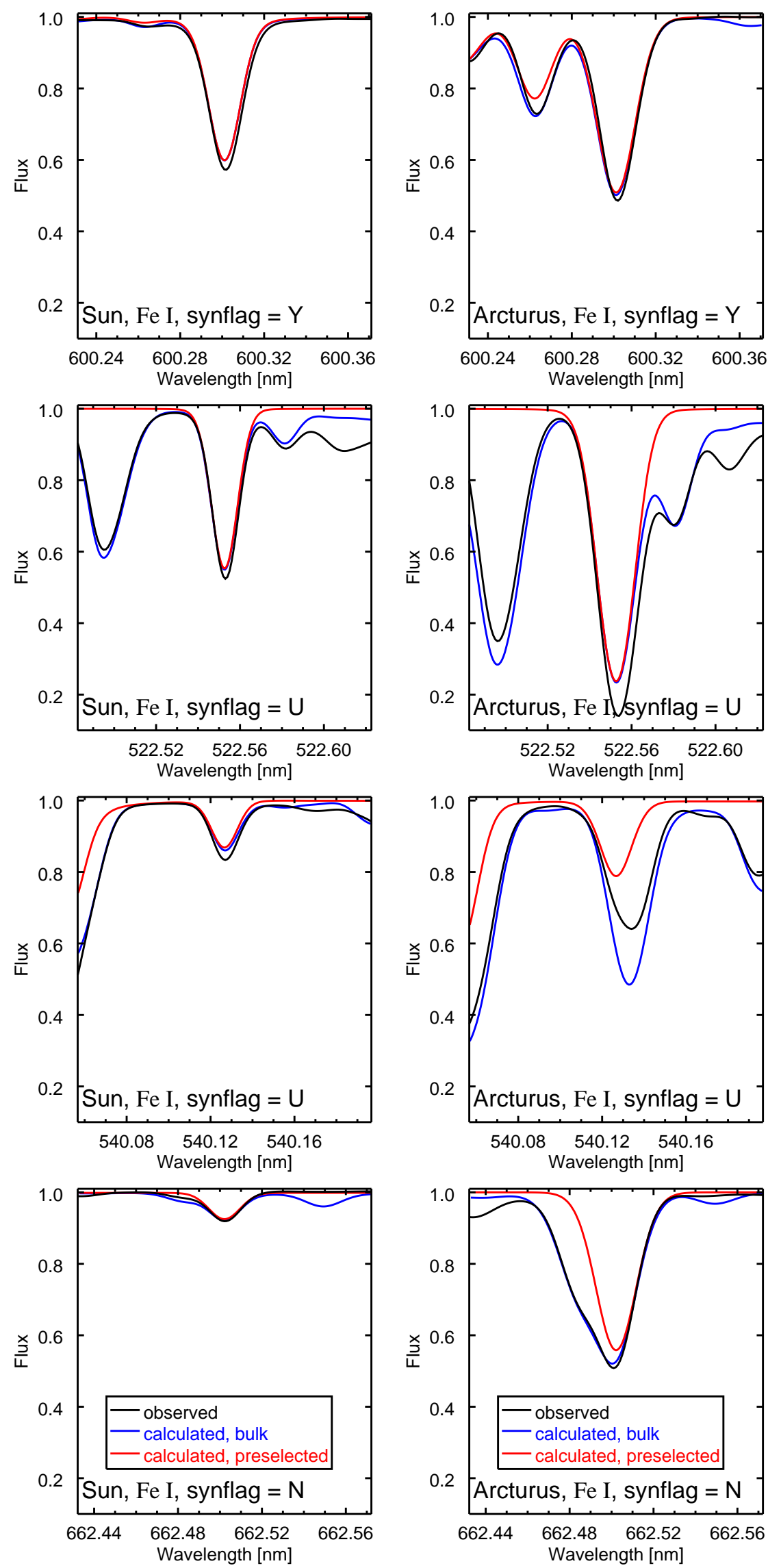

Fig. 4. Comparison of observed and calculated line profiles around four of the preselected Fe I lines with different synflag assignments for the Sun (left) and Arcturus (right). Black lines: observations, red lines: calculations including preselected spectral lines only, blue lines: calculations using the Gaia-ESO bulk line list, including preselected and background lines. All of these lines have $g f_{\text {flag }}=Y$. 

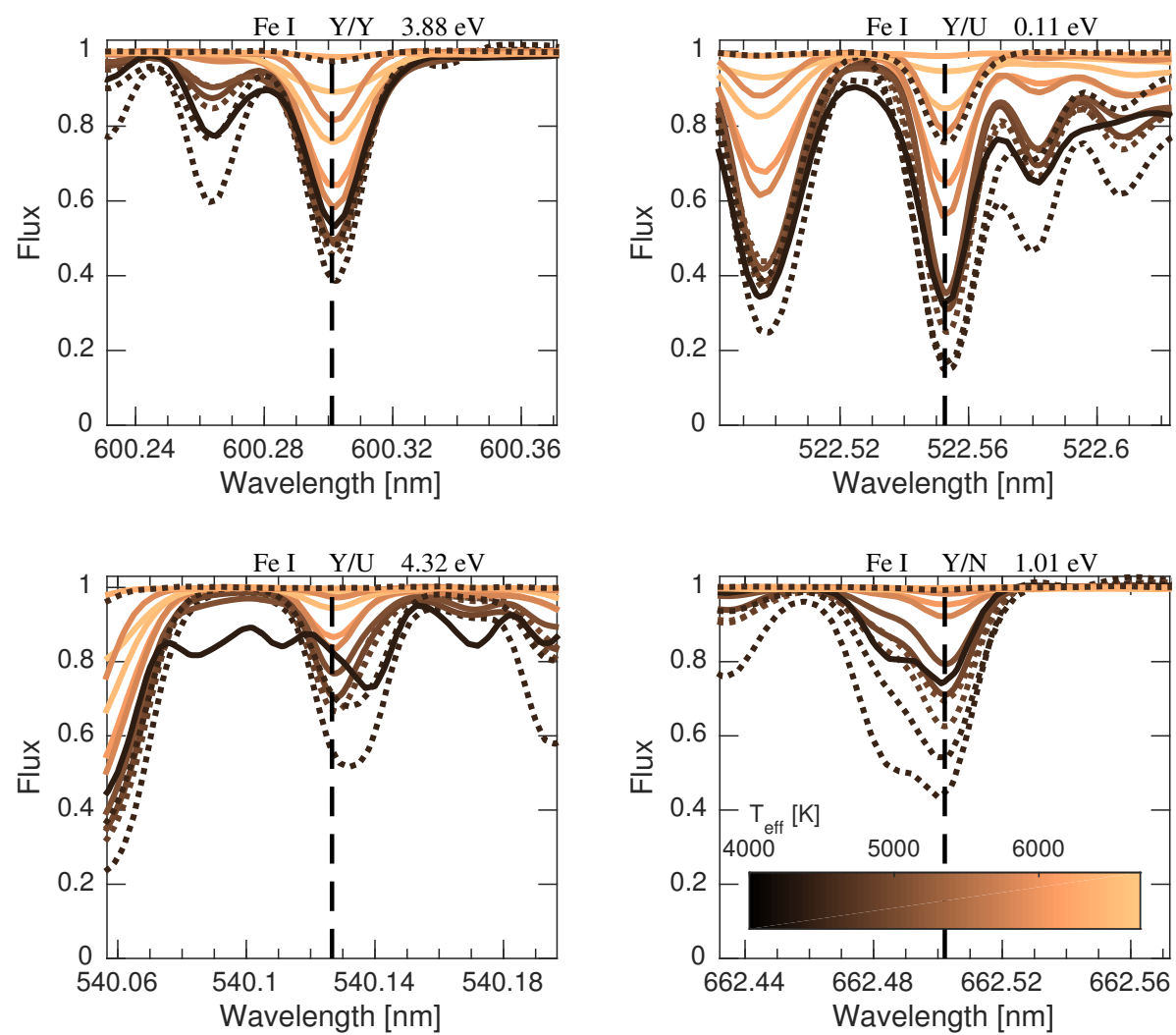

Fig. 5. Line profiles for four preselected Fe I lines generated from observed spectra of selected Gaia FGK benchmark stars (see Sect. 2.3). Quality flags and lower level energy are given at the top of each panel. The vertical dashed line indicates central wavelength. We note that $T_{\text {eff }}$ is coded by colour, solid lines are dwarfs, and dotted lines are giants.

Table 4. Molecular species, recommended dissociation energies $D_{00}$, and references for molecular transitions.

\begin{tabular}{|c|c|c|c|c|}
\hline & Isotopologues & $\begin{array}{l}D_{00} \\
{[\mathrm{eV}]}\end{array}$ & $D_{00}$ references & Line list references \\
\hline $\mathrm{CH}$ & ${ }^{12} \mathrm{CH},{ }^{13} \mathrm{CH}$ & 3.466 & Kumar et al. (1998) & Masseron et al. (2014) \\
\hline NH & & 3.420 & Tarroni et al. (1997) & T. Masseron (priv. comm.) \\
\hline $\mathrm{OH}$ & & 4.392 & Huber \& Herzberg (1979) & T. Masseron (priv. comm.) \\
\hline \multirow[t]{3}{*}{$\mathrm{C}_{2}$} & ${ }^{12} \mathrm{C}^{12} \mathrm{C}$ & 6.371 & Luo (2007) & Brooke et al. (2013, Swan) \\
\hline & ${ }^{12} \mathrm{C}^{13} \mathrm{C}$ & & & Ram et al. (2014, Swan) \\
\hline & ${ }^{13} \mathrm{C}^{13} \mathrm{C}$ & & & F. Quercy (priv. comm., Swan) \\
\hline \multirow[t]{2}{*}{$\mathrm{CN}$} & ${ }^{12} \mathrm{C}^{14} \mathrm{~N}$ & 7.738 & Huang et al. (1992) & Brooke et al. (2014) \\
\hline & ${ }^{12} \mathrm{C}^{15} \mathrm{~N},{ }^{13} \mathrm{C}^{14} \mathrm{~N}$ & & & Sneden et al. (2014) \\
\hline $\mathrm{MgH}$ & ${ }^{24} \mathrm{MgH},{ }^{25} \mathrm{MgH},{ }^{26} \mathrm{MgH}$ & 1.285 & Shayesteh et al. (2007) & $\begin{array}{l}\text { Hinkle et al. (2013, A-X), } \\
\text { T. Masseron (priv. comm., B-X) }\end{array}$ \\
\hline $\mathrm{SiH}$ & & 3.060 & Huber \& Herzberg (1979) & Kurucz (2010a) \\
\hline $\mathrm{CaH}$ & & 1.700 & Huber \& Herzberg (1979) & B. Plez (priv. comm.) \\
\hline $\mathrm{FeH}$ & & 1.598 & Dulick et al. (2003) & Dulick et al. (2003) \\
\hline $\mathrm{TiO}$ & ${ }^{46} \mathrm{TiO},{ }^{47} \mathrm{TiO},{ }^{48} \mathrm{TiO},{ }^{49} \mathrm{TiO},{ }^{50} \mathrm{TiO}$ & 6.87 & Naulin et al. (1997) & B. Plez (priv. comm.) \\
\hline VO & & 6.437 & B. Plez (priv. comm.) & B. Plez (priv. comm.) \\
\hline $\mathrm{ZrO}$ & ${ }^{90} \mathrm{ZrO},{ }^{91} \mathrm{ZrO},{ }^{92} \mathrm{ZrO},{ }^{94} \mathrm{ZrO},{ }^{96} \mathrm{ZrO}$ & 7.890 & B. Plez (priv. comm.) & B. Plez (priv. comm.) \\
\hline
\end{tabular}

Notes. ${ }^{(\dagger)}$ Excitation potentials were normalised to $0.0256 \mathrm{eV}$.

(Pancino et al. 2017). Reference values for metallicity (Jofré et al. 2014; Hawkins et al. 2016) and abundances of ten $\alpha$ - and iron-peak elements (Jofré et al. 2015) were determined from an analysis of high-resolution spectra. These works used a subset of the GES line list, where line selection was based on the quality flags ${ }^{17}$ and on low method-to-method dispersion. The standard

$\overline{17 \text { All lines with } g f_{-} \text {flag }=N}$ and Fe lines with synflag $=N$ were discarded. deviations of the abundances of the Fe I lines derived by six different methods for each star were between 0.01 and 0.03 dex. The abundances of the remaining elements were derived by eight different methods differentially (line-by-line) to different reference stars after grouping the stars by atmospheric parameters. The decrease in dispersion for the differential line abundances compared to the absolute abundances confirmed, among other things, the importance of hyperfine structure effects. For each element, lines commonly used for stars within groups of similar spectral 
types were identified and were referred to as 'golden lines'. In the case of Fe lines these were lines used for all stars within a group, and for the other elements a line was defined to be a golden line when it was analysed in at least $50 \%$ of the stars in the group. These include of the order of $100 \mathrm{Fe}$ I lines, as well as the Si I line at $568.448 \mathrm{~nm}$, which was used for all stars except two, to give some examples. For $\mathrm{V}$ and Co no golden lines were identified in metal-poor stars. A detailed discussion of golden lines can be found in Jofré et al. (2014, Sect. 6.3, Tables 4 and 5) and Jofré et al. (2015, Sect. 4.5 and online table ${ }^{18}$ ).

For the analysis of the UVES and GIRAFFE spectra observed within GES the different groups either used their own radiative transfer codes or a pre-computed grid of synthetic spectra. In both cases the Gaia-ESO line list was adopted. The grid contains 13784 high-resolution synthetic spectra for FGKMtype stars over the spectral ranges 420-690 and 845-895 nm. A wide range of metallicities (from $[\mathrm{M} / \mathrm{H}]=-5.0$ to $+1.0 \mathrm{dex}$ ) and $[\alpha / \mathrm{Fe}]$ enrichments (five values for each metallicity) is covered. For details see de Laverny et al. (2012).

An overview of the analysis procedure of UVES spectra observed within the GES is given in Smiljanic et al. (2014) for the case of FGK stars in the field and in older open clusters, and by Lanzafame et al. (2015) for the case of F- to M-type stars in the fields of young open clusters with ages of less than 100 Myr. Atmospheric parameters (mainly based on Fe lines) and abundances of up to 24 or 26 elements were derived by up to 13 and four different groups, respectively. Their results were subsequently combined into a homogeneous set of recommended values. The groups made their individual choices of line sub-sets to be used with their methods. For an illustration of the variation in line selection for Ca I see Fig. 4 in Jofré et al. (2019). In the first case, spectral lines measured by at least three groups were included in the combined abundances, and lines affected by blends as indicated by synflag were removed for species with 20 lines or more. In the second case, one of the groups based their selection on synflag for elements with $Z>28$, while the homogenisation was done without any further line selection. The precision of the abundances as indicated by the method-to-method dispersions was found to be similar in both cases, ranging from below 0.15 to 0.35 dex depending on the element. Stellar parameters and abundances of up to 11 chemical species were derived from GIRAFFE spectra observed within the GES by five different groups (A. Recio-Blanco, priv. comm., see also Worley et al. 2020). In this case the variation in line selection between groups is expected to be small because the number of lines in the relevant spectral range is low. Typical method-to-method dispersions in the combined and homogenised abundances were 0.04 dex.

Examples for studies of the structure and evolution of the Galactic bulge and disc based on the Gaia-ESO recommended metallicities and abundances are given in Williams et al. (2016), Bergemann et al. (2014), Recio-Blanco et al. (2014), and Mikolaitis et al. (2014). Typical mean uncertainties in abundances reported in these works are around $0.1 \mathrm{dex}$, which reflect the adoption of the Gaia-ESO line list, among other things. The abundance data allowed the authors to clearly distinguish between different sub-components in terms of metallicity and $\alpha$-element abundances, and to derive trends of abundances with other stellar properties such as age or galactocentric radius. Lind et al. (2015) identified one star among a few hundred halo stars that has most likely been ejected from a globular cluster,

18 https://cdsarc.unistra.fr/ftp/J/A+A/582/A81/

infoline.dat.gz based on a difference in $[\mathrm{Mg} / \mathrm{Fe}]$ abundance of 0.8 dex at a $4 \sigma$ significance compared to typical halo stars.

An example for advances in the area of open clusters made possible by GES spectra and the Gaia-ESO line list is given by three inner-disc clusters with ages of 0.3-1.5 Gyr. C, N, and $\mathrm{O}$ abundances with a typical precision of 0.05 dex were determined by Tautvaišiené et al. (2015), and abundances of 11 elements with average uncertainties of about 0.1 dex were determined by Magrini et al. (2015). In these works predictions made by models for stellar evolution and for Galactic chemical evolution were confirmed, and one cluster was found to be locally enriched by the Type II supernova explosion of a single star.

The EMBLA survey - an investigation of metal-poor stars in the Galactic bulge - is based on spectra obtained with a different instrument ${ }^{19}$, with similar resolution as the GES but covering a larger wavelength region (Howes et al. 2016). For the abundance analysis, the Gaia-ESO preselected line list was used as a starting point and was complemented by lines and data from other sources. There was a large overlap in line data between the two surveys for Fe II (mostly lines with $g f$ flag $=Y$ ), resulting in an average standard error of $\mathrm{Fe}$ line abundances of 0.06 dex. Other species with lines in common or with the same source for the $g f$-values were $\mathrm{Ca}$ I, Sc II, and Zn I, with mean uncertainties in abundances of $\sim 0.1 \mathrm{dex}$, and $\mathrm{Cr}$ I and Ba II with $\sim 0.2$ dex. Carbon abundances were determined from $\mathrm{CH}$ band heads using the same molecular data as the GES, with uncertainties of $\sim 0.2$ dex. These data allowed the authors to conclude that the fraction of carbon-enhanced stars might be lower in the bulge compared to the halo, and that some of the other elements behaved differently in the bulge stars than in halo stars.

\subsection{Data needs and recent developments}

This article describes version 6 of the Gaia-ESO line list, which was the last version produced within the GES consortium (mainly in 2014, with minor changes in 2016), and this is the version used for the final release of GES data products. The list is complete in the sense that it contains all atomic and molecular data available at the time of compilation for those transitions widely used for abundance analysis of FGK-type stars in the wavelength region of interest at the resolution of the FLAMESUVES spectrograph. This includes hyperfine structure and isotope splitting data, as well as references to the original sources for the data. The list contains about 200 lines (not counting HFS or IS components) of 24 species which have accurate $g f$-values and are free of blends in the spectra of the Sun and Arcturus, that is, both $g f$ flag and synflag $=Y$ (see column "Y/Y" in Table 1).

Prospective users of the line list are advised to update the $g f$-values with new data that may have become available since the compilation was done. Also, HFS and IS data are available for more species than considered relevant in the context of the GES, and users should add these according to the needs of their specific application. We note that for several species more recent calculations by R.L. Kurucz are available, in particular for C I, Si II, Ca I, Ti I, Ti II, V I, V II, Cr I, Cr II, Fe I (see discussion in Appendix B.16 for the latter). The Kurucz website provides HFS and IS components for many species.

Based on the presentation of available data in Appendix B we comment on those atomic species and lines for which future improvements of transition probability data should have the highest priority. We focus on the preselected lines with

19 The MIKE high-resolution spectrograph on Magellan's $6.5 \mathrm{~m}$ Clay telescope. 
synflag $=Y$ and $U$. A number of species have less than five such lines (see Table 1). Most of these have high-quality $g f$-values $(g f$ flag $=Y)$, including the light species Li I, C I, O I, and Si II, and the heavy species Zn I, Zr II, Ru I, Ba II, Pr II, Sm II, Eu II, Gd II, and Dy II. The exceptions are V II, Sr I, and Y I which have one or two lines with theoretical $g f$-values. However, these lines are extremely weak and probably blended in most stellar spectra and thus of low priority for abundance analysis.

Among the species with at least five lines with synflag $=Y$ or $U$ several have high-quality $g f$-values for all of those lines (Sc I, Cu I, Y II, Zr I, Mo I) or for the vast majority (>90\%, Ca I and Ti I). Another group of species has low-quality $g f$-values for more than $10 \%$, but less than $50 \%$ of the synflag $=Y$ or $U$ lines (Sc II, Ti II, V I, Cr I, Mn I, Co I, La II, Ce II, Nd II). These should be considered for laboratory measurements in the long term, but they are of lower immediate priority. We note that for the five Sc II lines with $g f \_l a g=N$ new theoretical $g f$-values based on branching fractions calculated using the relativistic HartreeFock method and life-times measured by Marsden et al. (1988) are available in Pehlivan Rhodin et al. (2017a). Finally, there are a number of species with low-quality $g f$-values for $50 \%$ or more of the synflag $=Y$ or $U$ lines, which should be given highest priority in current and future laboratory experiments.

The percentage is about 50 for both Fe I and Fe II. Specifically, 236 of $439 \mathrm{Fe}$ I lines would need improved $g f$-values. These are roughly evenly distributed over the whole wavelength range considered here and comprise a wide variety of transitions. They originate from lower levels which are preferentially odd (70\%) and belong to 36 different terms, of which the most frequent are $y^{5} F^{o}$ and $y^{3} F^{o}$ with energies of $\sim 4.25$ and $\sim 4.60 \mathrm{eV}$, respectively. The upper levels are more diverse with 75 different terms, of which the most frequent are $g^{5} F$ and $f^{5} G$ with energies around $6.6 \mathrm{eV}$. One third of these lines have synflag $=Y$. Almost half of them have theoretical $g f$-values by Kurucz (2007, which should be replaced by more recent calculations available at the Kurucz website), and most of the remaining ones have $g f$-values measured by May et al. (1974). In the case of Fe II 13 of 26 lines have purely theoretical $g f$-values. These are listed in Table A.3 and half of them have synflag $=Y$.

About 60 to $70 \%$ of the synflag $=Y$ or $U$ lines need improved $g f$-values for the species Na I, Mg I, Si I, Ca II, and Ni I. These lines are also listed in Table A.3 except for Mg I and Ni I. For Mg I new experimental oscillator strengths were recently published by Pehlivan Rhodin et al. (2017b), combining branching fractions measured from an FTS spectrum of a hollow cathode discharge lamp with radiative life-times from the literature and from their own calculations. They also published new theoretical oscillator strengths based on the multiconfiguration HartreeFock method. All but one of the eight $\mathrm{Mg}$ I lines in the Gaia-ESO line list needing improvement are included in this work, as well as the four lines which already had high-quality $g f$-values. Table A.4 lists both the data in the GES line list and the new data for all of these lines.

Numerous Ni I lines found over the whole wavelength range considered here are candidates for new experimental transition probabilities ( 53 of 83 with synflag $=Y$ or $U$ ). These lines are listed in Table A.5. Almost all of them currently have theoretical $g f$-values by Kurucz (2008). Nearly all of them originate from odd lower levels, which belong to 16 different terms, the most frequent one being $y^{3} F^{o}$ with energies of $\sim 4.2 \mathrm{eV}$. The upper levels belong to 14 different terms, the most frequent one being $3 \mathrm{~d}^{9}\left({ }^{2} D_{5 / 2}\right) 4 \mathrm{~d}^{2}\left[\frac{7}{2}\right]$ with energies around $6.1 \mathrm{eV}$. About $40 \%$ of these lines have synflag $=Y$.
Table 5. Lines of Al I, S I, and CrII with synflag $=Y$ or $U$, and with gfflag $=U$ or $N$

\begin{tabular}{lcll}
\hline \hline Species & Transition & $\begin{array}{l}\text { Wavelength } \\
(\AA)\end{array}$ & $\begin{array}{l}\text { Flags } \\
g f / \text { syn }\end{array}$ \\
\hline Al I & $a^{2} S_{0.5}-w^{2} P_{1.5}^{o}$ & 5557.063 & $U / U$ \\
Al I & $a^{2} S_{0.5}-x^{2} P_{1.5}^{o}$ & 6696.023 & $U / U$ \\
Al I & $a^{2} S_{0.5}-x^{2} P_{0.5}^{o}$ & 6698.673 & $U / Y$ \\
Al I & $a^{2} D_{1.5}-y^{2} F_{2.5}^{o}$ & 8772.865 & $U / U$ \\
Al I & $a^{2} D_{2.5}-y^{2} F_{6.5}^{o}$ & 8773.896 & $U / U$ \\
S I & $a^{5} P_{1}-x^{5} D_{0}^{o}$ & 6743.483 & $U / U$ \\
S I & $a^{5} P_{1}-x^{5} D_{2}^{o}$ & 6743.540 & $U / U$ \\
S I & $a^{5} P_{1}-x^{5} D_{1}^{o}$ & 6743.580 & $U / U$ \\
S I & $a^{5} P_{2}-x^{5} D_{1}^{o}$ & 6748.570 & $U / U$ \\
S I & $a^{5} P_{2}-x^{5} D_{2}^{o}$ & 6748.580 & $U / U$ \\
S I & $a^{5} P_{2}-x^{5} D_{3}^{o}$ & 6748.790 & $U / U$ \\
S I & $a^{5} P_{3}-x^{5} D_{4}^{o}$ & 6757.150 & $U / U$ \\
S I & $a^{5} P_{3}-y^{5} D_{4}^{o}$ & 8694.710 & $U / U$ \\
Cr II & $a^{4} F_{3.5}-z^{4} F_{3.5}^{o}$ & 4848.235 & $N / U$ \\
Cr II & $a^{4} F_{2.5}-z^{4} F_{1.5}^{o}$ & 4884.607 & $N / U$ \\
Cr II & $b^{4} F_{4.5}-z^{4} F_{4.5}^{o}$ & 5237.328 & $N / U$ \\
Cr II & $b^{4} F_{4.5}-z^{4} F_{3.5}^{o}$ & 5279.876 & $N / U$ \\
Cr II & $b^{4} P_{2.5}-z^{4} P_{2.5}^{o}$ & 5305.853 & $N / Y$ \\
Cr II & $b^{4} F_{1.5}-z^{4} F_{2.5}^{o}$ & 5310.686 & $N / U$ \\
Cr II & $b^{4} F_{2.5}-z^{4} F_{2.5}^{o}$ & 5313.563 & $N / U$ \\
Cr II & $b^{4} G_{4.5}-z^{4} F_{3.5}^{o}$ & 5502.067 & $N / U$ \\
\hline & & &
\end{tabular}

For Al I, S I, and Cr II none of the preselected lines with synflag $=Y$ or $U$ have high-quality $g f$-values (except for one $\mathrm{Cr}$ II line at $524.68 \mathrm{~nm}$ ). Experimental work on these species is highly needed, and the 21 lines concerned are listed in Table 5.

\subsection{Access to data}

The data comprising the Gaia-ESO line list in the wavelength ranges from 4750 to $6850 \AA$ and from 8488 to $8950 \AA$ are made available at the CDS. The atomic data are stored in a single table with one record for each transition. Hyperfine structure components and different isotopes are included as separate transitions, where applicable (see Sect. 2.5). HFS components belonging to the same fine structure transition can be identified by having the exact same label and $J$ value for both the lower and the upper levels. Both the preselected lines and the background line list are included. Preselected lines can be identified by having both non-empty $g f$ flag and synflag entries. Here we describe the contents of the data fields included for each transition.

Element Element symbol (e.g. Fe).

Ion Ionisation stage $(1=$ neutral, $2=$ singly ionised, $3=$ doubly ionised).

Isotope Isotope information for Element: 0 if only one isotope is present in the list, otherwise the baryon number is given.

lambda Wavelength of the transition in air, in units of $\AA$.

r_lambda Reference code for lambda.

loggf Logarithm (base 10) of the product of the oscillator strength of the transition and the statistical weight of the lower level. 
Table 6. Examples for atomic data.

\begin{tabular}{|c|c|c|c|c|c|c|c|c|c|}
\hline$\underline{E}$ & Ion & $\mathrm{I}$ & lambda & r_lambda & $\operatorname{logg} f$ & e_loggf & r_loggf & gf & syn \\
\hline $\mathrm{Li}$ & 1 & 7 & 6707.7635 & LN & -0.002 & 0.000 & 1998PhRvA. . 57.1652Y & $\mathrm{Y}$ & $\mathrm{U}$ \\
\hline $\mathrm{C}$ & 1 & $\theta$ & 6587.6100 & NIST10 & -1.021 & 0.000 & 1993AAS . . .99 . 179H & $\mathrm{Y}$ & $\mathrm{Y}$ \\
\hline 0 & 1 & $\theta$ & 6158.1858 & NIST10 & -0.296 & 0.000 & 1991JPhB . . 24.3943H & $\mathrm{Y}$ & $\mathrm{U}$ \\
\hline $\mathrm{Na}$ & 1 & $\theta$ & 5889.9509 & NIST 10 & 0.108 & 0.001 & 1996PhRvL . . 76.2862V & $\mathrm{Y}$ & $\mathrm{Y}$ \\
\hline $\mathrm{Mg}$ & 1 & $\theta$ & 5172.6843 & NIST 10 & -0.450 & 0.040 & ATJL & $\mathrm{Y}$ & $\mathrm{Y}$ \\
\hline Al & 1 & $\theta$ & 6698.6730 & WSM & -1.870 & 0.000 & $1995 J P h B \ldots 28.3485 M$ & $\mathrm{U}$ & $\mathrm{Y}$ \\
\hline Si & 1 & $\theta$ & 5690.4250 & GARZ & -1.773 & 0.000 & GARZ |BL & $\mathrm{Y}$ & $\mathrm{Y}$ \\
\hline Si & 2 & $\theta$ & 6347.1087 & $\mathrm{~K} 12$ & 0.169 & 0.000 & $\mathrm{~S}-\mathrm{G}+\mathrm{BBC}+\mathrm{MER}$ & $\mathrm{Y}$ & $\mathrm{U}$ \\
\hline $\mathrm{S}$ & 1 & $\theta$ & 6743.4832 & KQ4 & -1.310 & 0.000 & $2006 \mathrm{JPhB} \ldots 39.2861 \mathrm{Z}+\mathrm{GESMCHF}$ & $\mathrm{U}$ & $\mathrm{U}$ \\
\hline $\mathrm{Ca}$ & 1 & $\theta$ & 5260.3870 & $\mathrm{SR}+\mathrm{Sm}$ & -1.719 & 0.011 & SR & $\mathrm{Y}$ & $\mathrm{Y}$ \\
\hline $\mathrm{Ca}$ & 2 & 0 & 8542.0910 & $\mathrm{~T}$ & -0.463 & 0.000 & $\mathrm{~T}$ & $\mathrm{Y}$ & $\mathrm{Y}$ \\
\hline Sc & 1 & $\theta$ & 5356.0868 & LD & -0.189 & 0.000 & LD & $\mathrm{Y}$ & $\mathrm{Y}$ \\
\hline Sc & 2 & 0 & 5657.8960 & LD & -0.603 & 0.000 & LD & $\mathrm{Y}$ & $\mathrm{Y}$ \\
\hline $\mathrm{Ti}$ & 1 & $\theta$ & 4758.1178 & LGWSC & 0.510 & 0.000 & $2013 \mathrm{ApJS} .205 \ldots 11 \mathrm{~L}$ & $\mathrm{Y}$ & $\mathrm{Y}$ \\
\hline $\mathrm{Ti}$ & 2 & 0 & 5418.7678 & WLSC & -2.130 & 0.000 & $2013 \mathrm{ApJS} . .208 \ldots 27 \mathrm{~W}$ & $\mathrm{Y}$ & $\mathrm{Y}$ \\
\hline V & 1 & (1) & 5604.9012 & K09 & -1.644 & 0.000 & 1985AA .. 153 . 109W & $\mathrm{Y}$ & $\mathrm{Y}$ \\
\hline $\mathrm{V}$ & 2 & $\theta$ & 6028.2680 & $\mathrm{~K} 10$ & -2.122 & 0.000 & $\mathrm{~K} 10$ & $\mathrm{~N}$ & $\mathrm{U}$ \\
\hline $\mathrm{Cr}$ & 1 & $\theta$ & 4936.3350 & WLHK & -0.250 & 0.000 & SLS & $\mathrm{Y}$ & $\mathrm{Y}$ \\
\hline $\mathrm{Cr}$ & 2 & $\theta$ & 5246.7680 & PGBH & -2.466 & 0.000 & PGBH & $\mathrm{Y}$ & $\mathrm{U}$ \\
\hline Mn & 1 & 0 & 5394.6191 & $\mathrm{~K} \otimes 7$ & -4.070 & 0.000 & 1984MNRAS. $208 \ldots$ 147B & $\mathrm{Y}$ & $\mathrm{Y}$ \\
\hline $\mathrm{Fe}$ & 1 & $\theta$ & 4802.8797 & BWL & -1.514 & 0.051 & BWL & $\mathrm{Y}$ & $\mathrm{Y}$ \\
\hline $\mathrm{Fe}$ & 2 & 0 & 4923.9212 & K13 & -1.260 & 0.000 & 2009AA . .497 .611M & $\mathrm{Y}$ & $\mathrm{Y}$ \\
\hline Co & 1 & $\theta$ & 5331.4121 & K08 & -2.461 & 0.000 & 1999ApJS. . 122 . 557N & $\mathrm{Y}$ & $\mathrm{Y}$ \\
\hline $\mathrm{Ni}$ & 1 & $\theta$ & 5424.6450 & K08 & -2.770 & 0.000 & 1985JQSRT . . 33 . .307D & $\mathrm{Y}$ & $\mathrm{Y}$ \\
\hline $\mathrm{Cu}$ & 1 & 65 & 5782.0385 & $\mathrm{~K} 12$ & -2.817 & 0.000 & KR|1989ZPhyD . . 11 . 287C & $\mathrm{Y}$ & $\mathrm{Y}$ \\
\hline $\mathrm{Zn}$ & 1 & $\theta$ & 4810.5280 & Wa & -0.160 & 0.000 & 1980AA . . .84 . 361B|1980ZPhyA.298 . 249K & $\mathrm{Y}$ & $\mathrm{U}$ \\
\hline $\mathrm{Sr}$ & 1 & $\theta$ & 6791.0160 & GC & -0.730 & 0.000 & GC & $\mathrm{Y}$ & $\mathrm{U}$ \\
\hline $\mathrm{Y}$ & 1 & $\theta$ & 6222.5775 & K06 & -1.452 & 0.000 & K06 & $\mathrm{N}$ & $\mathrm{U}$ \\
\hline $\mathrm{Y}$ & 2 & 0 & 4883.6821 & $\mathrm{~K} 11$ & 0.190 & 0.000 & BBEHL & $\mathrm{Y}$ & $\mathrm{Y}$ \\
\hline $\mathrm{Zr}$ & 1 & $\theta$ & 6127.4400 & BGHL & -1.060 & 0.000 & BGHL & $\mathrm{Y}$ & $\mathrm{Y}$ \\
\hline $\mathrm{Zr}$ & 2 & 0 & 5112.2700 & LNAJ & -0.850 & 0.000 & LNAJ & $\mathrm{Y}$ & $\mathrm{U}$ \\
\hline $\mathrm{Nb}$ & 1 & $\theta$ & 5095.2930 & DLa & -1.048 & 0.000 & 1986JQSRT . . 35 . .281D & $\mathrm{Y}$ & $\mathrm{N}$ \\
\hline Mo & 1 & $\theta$ & 5751.4080 & $\mathrm{WBb}$ & -1.014 & 0.000 & $\mathrm{WBb}$ & $\mathrm{Y}$ & $\mathrm{Y}$ \\
\hline $\mathrm{Ru}$ & 1 & $\theta$ & 4869.1530 & WSL & -0.830 & 0.000 & WSL & $\mathrm{Y}$ & $\mathrm{U}$ \\
\hline $\mathrm{Ba}$ & 2 & 135 & 5853.6663 & MW & -0.907 & 0.000 & 1992AA ..255 . 457D & $\mathrm{Y}$ & $\mathrm{Y}$ \\
\hline $\mathrm{La}$ & 2 & 139 & 4804.0020 & LBS & -2.092 & 0.000 & LBS & $\mathrm{Y}$ & $\mathrm{U}$ \\
\hline $\mathrm{Ce}$ & 2 & $\theta$ & 5274.2290 & LSCI & 0.130 & 0.000 & LSCI & $\mathrm{Y}$ & $\mathrm{Y}$ \\
\hline $\operatorname{Pr}$ & 2 & 141 & 5322.6729 & ILW & -2.870 & 0.000 & 2007Phys...76. 577L & $\mathrm{Y}$ & $\mathrm{Y}$ \\
\hline $\mathrm{Nd}$ & 2 & 143 & 4914.3624 & HLSC & -1.226 & 0.000 & HLSC & $\mathrm{Y}$ & $\mathrm{U}$ \\
\hline Sm & 2 & 149 & 4836.6422 & LD-HS & -2.758 & 0.000 & LD-HS & $\mathrm{Y}$ & $\mathrm{U}$ \\
\hline $\mathrm{Eu}$ & 2 & 153 & 5818.7119 & LWHS & -2.572 & 0.000 & LWHS & $\mathrm{Y}$ & $\mathrm{U}$ \\
\hline $\mathrm{Gd}$ & 2 & $\theta$ & 4865.0410 & DLSC & -0.870 & 0.000 & DLSC & $\mathrm{Y}$ & $\mathrm{U}$ \\
\hline Dy & 2 & $\theta$ & 5169.6900 & WLN & -1.950 & 0.000 & WLN & $\mathrm{Y}$ & $\mathrm{U}$ \\
\hline
\end{tabular}

Notes. Full table available at CDS. Column headers give field names, where E, I, gf, syn, R, S, and V are Element, Isotope, gf_flag, synflag, Rad_damp, Sta_damp, and Vdw_damp, respectively. See Sect. 4.3 and ReadMe file at CDS for description of fields.

e_loggf Uncertainty in loggf for experimental $g f$-values if available.

r_loggf Reference code for loggf. This field may contain several labels combined with + or $I$. When the labels are combined with + then logg $f$ is the average from more than one source, while I means that relative $g f$-values from the first source were re-normalised to an absolute scale using accurate life-time measurements from the second source (see the respective subsection on $\mathrm{Si}, \mathrm{Ti}, \mathrm{Fe}, \mathrm{Cu}$, and $\mathrm{Zn}$ in Appendix B).

$g f$ flag Flag indicating the relative quality for loggf (usage recommendation, values $Y / U / N$, see Sect. 2.2), for preselected lines only. synflag Flag indicating the blending quality of the spectral line for synthesis (usage recommendation based on spectra of the Sun and Arcturus, values $Y / U / N$, see Sect. 2.7), for preselected lines only.

Label_low A string of characters specifying the electronic configuration and the term designation for the lower energy level. Taken from the VALD database, which follows the notation adopted by the NIST Atomic Spectra Database ${ }^{20}$.

J_low Total angular momentum quantum number $J$ for the lower level.

E_low Lower level energy in units of $\mathrm{eV}$.

${ }^{20}$ See http://www . astro.uu.se/valdwiki/AtomicLevel 
Table 6. continued.

\begin{tabular}{|c|c|c|c|c|c|c|c|c|c|c|c|c|c|}
\hline $\mathrm{E}$ & Ion & J_low & E_low & r_E_low & J_up & E_up & r_E_up & $\mathrm{R}$ & r_Rad_damp & S & r_Sta_damp & V & r_Vdw_damp \\
\hline $\mathrm{Li}$ & 1 & 0.5 & 0.000 & LN & 1.5 & 1.848 & LN & 7.56 & CDROM18 & -5.78 & CDROM18 & 346.236 & $\mathrm{BA}-\mathrm{J} ; \mathrm{BPM}$ \\
\hline C & 1 & 1.0 & 8.537 & NIST10 & 1.0 & 10.419 & NIST 10 & 8.10 & $\mathrm{~K} 10$ & -3.44 & $\mathrm{~K} 10$ & 1953.319 & $\mathrm{BA}-\mathrm{J} ; \mathrm{BPM}$ \\
\hline 0 & 1 & 3.0 & 10.741 & NIST10 & 10.0 & 12.754 & NIST 10 & 7.62 & CDROM18 & -3.96 & CDROM18 & 1915.322 & $\mathrm{BA}-\mathrm{J} ; \mathrm{BPM}$ \\
\hline $\mathrm{Na}$ & 1 & 0.5 & 0.000 & NIST 10 & 1.5 & 2.104 & NIST 10 & 7.80 & CDROM18 & -5.64 & CDROM18 & 407.273 & $\mathrm{BA}-\mathrm{J} ; \mathrm{BPM}$ \\
\hline $\mathrm{Mg}$ & 1 & 1.0 & 2.712 & NIST10 & 1.0 & 5.108 & NIST 10 & 7.99 & CDROM18 & -5.47 & CDROM18 & 729.238 & $\mathrm{BA}-\mathrm{J} ; \mathrm{BPM}$ \\
\hline $\mathrm{Al}$ & 1 & 0.5 & 3.143 & WSM & 0.5 & 4.993 & WSM & 0.00 & & 0.00 & & 0.000 & \\
\hline Si & 1 & 1.0 & 4.930 & GARZ & 1.0 & 7.108 & GARZ & 8.54 & K07 & -4.57 & K07 & 1770.220 & $\mathrm{BA}-\mathrm{J} ; \mathrm{BPM}$ \\
\hline Si & 2 & 0.5 & 8.121 & $\mathrm{~K} 12$ & 1.5 & 10.074 & $\mathrm{~K} 12$ & 9.08 & $\mathrm{~K} 12$ & -5.68 & $\mathrm{~K} 12$ & -7.690 & $\mathrm{~K} 12$ \\
\hline$S$ & 1 & 1.0 & 7.866 & K04 & 0.0 & 9.704 & KQ4 & 7.60 & KO4 & -4.54 & KO4 & 0.000 & \\
\hline $\mathrm{Ca}$ & 1 & 1.0 & 2.521 & $\mathrm{SR}+\mathrm{Sm}$ & 2.0 & 4.878 & $\mathrm{SR}+\mathrm{Sm}$ & 7.90 & K07 & -5.76 & K07 & 421.260 & $\mathrm{BA}-\mathrm{J} ; \mathrm{BPM}$ \\
\hline $\mathrm{Ca}$ & 2 & 2.5 & 1.700 & $\mathrm{~T}$ & 1.5 & 3.151 & $\mathrm{~T}$ & 8.21 & $\mathrm{~K} 10$ & -5.70 & $\mathrm{~K} 10$ & 291.275 & $\mathrm{BA}-\mathrm{J} ; \mathrm{BPM}$ \\
\hline Sc & 1 & 3.5 & 1.865 & LD & 2.5 & 4.179 & LD & 8.19 & KO9 & -5.82 & KO9 & 412.271 & $\mathrm{BA}-\mathrm{J} ; \mathrm{BPM}$ \\
\hline Sc & 2 & 2.0 & 1.507 & LD & 2.0 & 3.698 & LD & 8.18 & K09 & -6.55 & KO9 & -7.860 & K09 \\
\hline $\mathrm{Ti}$ & 1 & 5.0 & 2.249 & LGWSC & 5.0 & 4.854 & LGWSC & 8.08 & $\mathrm{~K} 10$ & -6.04 & $\mathrm{~K} 10$ & 326.246 & BA-J ; BPM \\
\hline $\mathrm{Ti}$ & 2 & 2.5 & 1.582 & WLSC & 2.5 & 3.869 & WLSC & 8.15 & K10 & -6.59 & $\mathrm{~K} 10$ & -7.850 & K10 \\
\hline $\mathrm{V}$ & 1 & 0.5 & 1.043 & K09 & 1.5 & 3.255 & K०9 & 8.02 & K०9 & -5.96 & K09 & 343.237 & $\mathrm{BA}-\mathrm{J} ; \mathrm{BPM}$ \\
\hline V & 2 & 1.0 & 2.491 & $\mathrm{~K} 10$ & 2.0 & 4.547 & $\mathrm{~K} 10$ & 8.33 & K10 & -6.45 & $\mathrm{~K} 10$ & -7.870 & K10 \\
\hline $\mathrm{Cr}$ & 1 & 3.0 & 3.113 & WLHK & 4.0 & 5.624 & WLHK & 7.91 & $\mathrm{~K} 10$ & -5.97 & $\mathrm{~K} 10$ & 342.245 & $\mathrm{BA}-\mathrm{J} ; \mathrm{BPM}$ \\
\hline $\mathrm{Cr}$ & 2 & 0.5 & 3.714 & PGBH & 1.5 & 6.076 & PGBH & 8.32 & $\mathrm{~K} 10$ & -6.49 & $\mathrm{~K} 10$ & 186.227 & $\mathrm{BA}-\mathrm{J} ; \mathrm{BPM}$ \\
\hline Mn & 1 & 2.5 & 0.000 & K07 & 3.5 & 2.298 & K07 & 4.29 & K07 & -6.27 & K07 & 219.252 & $\mathrm{BA}-\mathrm{J} ; \mathrm{BPM}$ \\
\hline $\mathrm{Fe}$ & 1 & 3.0 & 3.642 & $\mathrm{~K} 07$ & 2.0 & 6.222 & K07 & 7.96 & $\mathrm{~K} 07$ & -6.03 & $\mathrm{~K} 07$ & 356.244 & $\mathrm{BA}-\mathrm{J} ; \mathrm{BPM}$ \\
\hline $\mathrm{Fe}$ & 2 & 2.5 & 2.891 & $\mathrm{~K} 13$ & 1.5 & 5.408 & $\mathrm{~K} 13$ & 8.49 & $\mathrm{~K} 13$ & -6.53 & $\mathrm{~K} 13$ & 175.202 & $\mathrm{BA}-\mathrm{J} ; \mathrm{BPM}$ \\
\hline Co & 1 & 0.5 & 1.785 & K08 & 1.5 & 4.110 & K08 & 8.12 & K08 & -6.15 & $\mathrm{~K} 08$ & 303.260 & $\mathrm{BA}-\mathrm{J} ; \mathrm{BPM}$ \\
\hline $\mathrm{Ni}$ & 1 & 1.0 & 1.951 & K08 & 2.0 & 4.236 & K08 & 8.07 & K08 & -6.16 & K08 & 232.270 & $\mathrm{BA}-\mathrm{J} ; \mathrm{BPM}$ \\
\hline $\mathrm{Cu}$ & 1 & 1.5 & 1.642 & $\mathrm{~K} 12$ & 0.5 & 3.786 & $\mathrm{~K} 12$ & 8.18 & $\mathrm{~K} 12$ & -6.07 & $\mathrm{~K} 12$ & -7.790 & K12 \\
\hline $\mathrm{Zn}$ & 1 & 2.0 & 4.078 & Wa & 1.0 & 6.655 & Wa & 0.00 & & 0.00 & & 676.238 & $\mathrm{BA}-\mathrm{J} ; \mathrm{BPM}$ \\
\hline $\mathrm{Sr}$ & 1 & 0.0 & 1.775 & GC & 1.0 & 3.600 & GC & 0.00 & & 0.00 & & 0.000 & \\
\hline $\mathrm{Y}$ & 1 & 1.5 & 0.000 & K06 & 2.5 & 1.992 & K06 & 6.96 & K06 & -5.97 & K06 & -7.740 & K06 \\
\hline $\mathrm{Y}$ & 2 & 4.0 & 1.084 & $\mathrm{~K} 11$ & 3.0 & 3.622 & $\mathrm{~K} 11$ & 8.47 & $\mathrm{~K} 11$ & -6.40 & K11 & -7.760 & $\mathrm{~K} 11$ \\
\hline $\mathrm{Zr}$ & 1 & 4.0 & 0.154 & BGHL & 4.0 & 2.177 & BGHL & 0.00 & & 0.00 & & 260.244 & $\mathrm{BA}-\mathrm{J} ; \mathrm{BPM}$ \\
\hline $\mathrm{Zr}$ & 2 & 1.5 & 1.665 & LNAJ & 1.5 & 4.090 & LNA] & 0.00 & & 0.00 & & 0.000 & \\
\hline $\mathrm{Nb}$ & 1 & 3.5 & 0.086 & DLa & 3.5 & 2.519 & DLa & 0.00 & & 0.00 & & 0.000 & \\
\hline Mo & 1 & 2.0 & 1.420 & $\mathrm{WBb}$ & 2.0 & 3.575 & $\mathrm{WBb}$ & 0.00 & & 0.00 & & 0.000 & \\
\hline $\mathrm{Ru}$ & 1 & 4.0 & 0.928 & WSL & 4.0 & 3.473 & WSL & 0.00 & & 0.00 & & 0.000 & \\
\hline $\mathrm{Ba}$ & 2 & 1.5 & 0.604 & MW & 1.5 & 2.722 & MW & 0.00 & & 0.00 & & 365.264 & $\mathrm{BA}-\mathrm{J} ; \mathrm{BPM}$ \\
\hline $\mathrm{La}$ & 2 & 1.0 & 0.235 & LBS & 1.0 & 2.815 & LBS & 0.00 & & 0.00 & & 0.000 & \\
\hline $\mathrm{Ce}$ & 2 & 6.5 & 1.044 & LSCI & 5.5 & 3.395 & LSCI & 0.00 & & 0.00 & & 0.000 & \\
\hline $\operatorname{Pr}$ & 2 & 6.0 & 0.483 & ILW & 5.0 & 2.811 & ILW & 0.00 & & 0.00 & & 0.000 & \\
\hline $\mathrm{Nd}$ & 2 & 5.5 & 0.380 & HLSC & 4.5 & 2.902 & HLSC & 0.00 & & 0.00 & & 0.000 & \\
\hline $\mathrm{Sm}$ & 2 & 2.5 & 0.104 & LD-HS & 1.5 & 2.667 & LD-HS & 0.00 & & 0.00 & & 0.000 & \\
\hline $\mathrm{Eu}$ & 2 & 2.0 & 1.230 & LWHS & 3.0 & 3.361 & LWHS & 0.00 & & 0.00 & & 0.000 & \\
\hline $\mathrm{Gd}$ & 2 & 2.5 & 1.157 & DLSC & 1.5 & 3.704 & DLSC & 0.00 & & 0.00 & & 0.000 & \\
\hline Dy & 2 & 7.5 & 0.103 & WLN & 7.5 & 2.500 & WLN & 0.00 & & 0.00 & & 0.000 & \\
\hline
\end{tabular}

r_E_low Reference code for E_low.

Label_up Configuration and term label for the upper energy level.

J_up Total angular momentum quantum number $J$ for the upper level.

E_up Upper level energy in units of eV.

r_E_up Reference code for E_up.

Rad_damp Logarithm of the radiative damping width in units of $\mathrm{rad} \mathrm{s}^{-1}$ (see Sect. 2.6).

r_Rad_damp Reference code for Rad_damp.

Sta_damp Logarithm of the Stark broadening width per unit perturber number density at $10000 \mathrm{~K}$, in units of $\mathrm{rad} \mathrm{s}^{-1} \mathrm{~cm}^{3}$ (see Sect. 2.6).
r_Sta_damp Reference code for Sta_damp.

Vdw_damp Van der Waals broadening parameter (see Sect. 2.6 and Appendices E-H). Values greater than zero were obtained from $\mathrm{ABO}$ theory and are expressed in a packed notation where the integer component is the broadening cross-section, $\sigma$, in atomic units, and the decimal component is the dimensionless velocity parameter, $\alpha$. Values less than zero are the logarithm of the broadening width per unit perturber number density at $10000 \mathrm{~K}$ in units of $\mathrm{rad} \mathrm{s}^{-1} \mathrm{~cm}^{3}$.

r_Vdw_damp Reference code for Vdw_damp.

The atomic data are available in their entirety in a machinereadable form at the CDS. Table 6 lists excerpted data fields from the CDS data table containing all the atomic line parameters, for guidance regarding the content of the CDS table. When 
A\&A 645, A106 (2021)

Table 7. Examples for molecular data.

\begin{tabular}{|c|c|c|c|c|c|c|c|c|c|c|c|c|c|c|}
\hline E1 & E2 & I1 & $\mathrm{I} 2$ & lambda & $\operatorname{logg} f$ & E_low & E_up & $\mathrm{R}$ & Sl & $\mathrm{Su}$ & v_low & v_up & Branch & r_mol \\
\hline C & $\mathrm{H}$ & 12 & 1 & 750.010 & 3.113 & 1.208 & .818 & 6.522 & $\mathrm{X}$ & A & 3 & 2 & $\mathrm{R} 2 \mathrm{f}(11.5)$ & 014AA . . 571A . 47M \\
\hline C & $\mathrm{H}$ & 12 & 1 & 4750.088 & -3.117 & 1.208 & 3.818 & 6.522 & $\mathrm{X}$ & A & 3 & 2 & $\mathrm{R} 1 \mathrm{e}(12.5)$ & $2014 \mathrm{AA} \ldots 571 \mathrm{~A} \ldots 47 \mathrm{M}$ \\
\hline C & $\mathrm{H}$ & 12 & 1 & 4750.050 & -5.854 & 1.198 & 3.808 & 14.283 & $\mathrm{X}$ & B & 2 & 1 & $\mathrm{R} 12(17.5)$ & $2014 \mathrm{AA} \ldots 571 \mathrm{~A} \ldots 47 \mathrm{M}$ \\
\hline$c$ & $\mathrm{H}$ & 13 & 1 & 4750.085 & -3.482 & 2.095 & 4.705 & 6.290 & $\mathrm{X}$ & A & 4 & 3 & $\mathrm{R} 1 \mathrm{e}(24.5)$ & $2014 \mathrm{AA} \ldots 571 \mathrm{~A} \ldots 47 \mathrm{M}$ \\
\hline $\mathrm{N}$ & $\mathrm{H}$ & 14 & 1 & 4750.087 & -6.597 & 1.136 & 3.746 & 6.391 & $\mathrm{X}$ & $\mathrm{A}$ & 3 & 0 & $\operatorname{R} 1 \mathrm{e}(5.0)$ & T.Masseron.priv.comm \\
\hline $\mathrm{N}$ & $\mathrm{H}$ & 14 & 1 & 4750.587 & -5.503 & 1.815 & 4.424 & 6.336 & $\mathrm{X}$ & A & 3 & $\theta$ & $\operatorname{P3e}(19.0)$ & T.Masseron.priv.comm \\
\hline $\mathrm{N}$ & $\mathrm{H}$ & 14 & 1 & 4750.777 & -7.782 & 1.815 & .424 & 6.330 & $\mathrm{X}$ & $\mathrm{A}$ & 3 & $\theta$ & Q23e (19.0) & T.Masseron.priv.comm \\
\hline $\mathrm{N}$ & $\mathrm{H}$ & 14 & 1 & 4750.806 & -5.460 & 1.815 & 4.424 & 6.330 & $\mathrm{X}$ & A & 3 & $\theta$ & $\operatorname{P} 2 f(20 . \theta)$ & T.Masseron.priv.comm \\
\hline 0 & $\mathrm{H}$ & 16 & 1 & 4751.112 & -6.767 & 2.617 & 5.226 & 8.960 & $\mathrm{X}$ & $\mathrm{A}$ & 5 & 2 & $\mathrm{P} 12 \mathrm{e}(17.5)$ & T.Masseron.priv.comm \\
\hline 0 & $\mathrm{H}$ & 16 & 1 & 4751.586 & -6.010 & 3.566 & 6.175 & 9.294 & $\mathrm{X}$ & $\mathrm{A}$ & 4 & $\theta$ & $\mathrm{R} 1 \mathrm{e}(33.5)$ & T.Masseron.priv.comm \\
\hline 0 & $\mathrm{H}$ & 16 & 1 & 4751.861 & -7.328 & 2.498 & 5.107 & 7.083 & $\mathrm{X}$ & $\mathrm{A}$ & 4 & 1 & $\mathrm{P} 12 \mathrm{e}($ & iv. comm \\
\hline 0 & $\mathrm{H}$ & 16 & 1 & 4752.954 & -8.063 & 3.566 & 6.174 & 9.149 & $\mathrm{X}$ & $\mathrm{A}$ & 4 & $\theta$ & $\mathrm{Q} 21 \mathrm{e}(33.5)$ & on.priv.comm \\
\hline C & $\mathrm{C}$ & 12 & 12 & 4750.079 & -3.539 & 1.858 & 4.468 & 0.000 & $\mathrm{a}$ & $\mathrm{d}$ & 9 & 10 & 9) & $124 \ldots 11 \mathrm{~B}$ \\
\hline C & $\mathrm{C}$ & 12 & 12 & 4750.130 & -6.061 & 1.608 & 4.218 & 0.000 & $\mathrm{a}$ & $\mathrm{d}$ & 8 & 9 & $\mathrm{pR} 13(20)$ & 2013JQSRT.124 ...11B \\
\hline C & $\mathrm{C}$ & 12 & 13 & 4750.040 & -6.833 & 0.012 & 2.622 & 0.000 & $\mathrm{a}$ & $\mathrm{d}$ & $\theta$ & 1 & $\mathrm{oQ13}(06)$ & 2014 ApJS. .211...5R \\
\hline C & $\mathrm{C}$ & 13 & 13 & 4750.000 & -1.086 & 0.193 & 2.803 & 0.000 & $\mathrm{a}$ & $\mathrm{d}$ & $\theta$ & 1 & $\mathrm{P} 2(6)$ & Quercy.priv.comm \\
\hline C & $\mathrm{N}$ & 12 & 14 & 4750.026 & -4.015 & 0.886 & 3.496 & 0.000 & $\mathrm{X}$ & A & 11 & 3 & Qc (235) & 2014 ApJS $.210 \ldots 23 B$ \\
\hline C & $\mathrm{N}$ & 12 & 14 & 4750.043 & -5.373 & 0.007 & 2.617 & 0.000 & $\mathrm{X}$ & $\mathrm{A}$ & 7 & $\theta$ & Qc $(045)$ & 2014ApJS . 210..23B \\
\hline C & $\mathrm{N}$ & 12 & 15 & 4879.879 & -3.393 & 1.537 & 4.077 & 0.000 & $\mathrm{X}$ & B & $\theta$ & 3 & $\mathrm{Rb}(595)$ & 2014 ApJS $.214 \ldots 26 \mathrm{~S}$ \\
\hline C & $\mathrm{N}$ & 13 & 14 & 4750.006 & -3.602 & 2.119 & 4.729 & 0.000 & $\mathrm{X}$ & A & 19 & 9 & $\operatorname{Rc}(015)$ & 2014ApJS. .214...26S \\
\hline $\mathrm{Mg}$ & $\mathrm{H}$ & 24 & 1 & 4750.116 & -0.939 & 0.467 & 3.077 & 0.000 & $\mathrm{x}$ & A & 3 & 2 & $R 2 f(13.5)$ & $2013 \mathrm{ApJS} .207 \ldots 26 \mathrm{H}$ \\
\hline $\mathrm{Mg}$ & $\mathrm{H}$ & 24 & 1 & 4750.144 & -1.713 & 1.086 & 3.696 & 0.000 & $\mathrm{X}$ & B & 5 & 9 & $\operatorname{Re}(22.5)$ & eron.priv.comm \\
\hline $\mathrm{Mg}$ & $\mathrm{H}$ & 25 & 1 & 5007.964 & 0.412 & 0.662 & 3.137 & 0.000 & $\mathrm{X}$ & $\mathrm{A}$ & $\theta$ & $\theta$ & 1.5) & JS . .207 . .26H \\
\hline $\mathrm{Mg}$ & $\mathrm{H}$ & 26 & 1 & 5008.187 & 0.412 & 0.662 & 3.137 & 0.000 & $\mathrm{X}$ & 12 & $\theta$ & 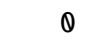 & $\mathrm{R} 2 \mathrm{e}$ & JS. . 207 . . 26H \\
\hline Si & $\mathrm{H}$ & 28 & 1 & 4750.010 & -2.852 & 1.375 & 3.985 & 0.000 & $\mathrm{X}$ & A & 4 & 4 & $\mathrm{Q} 2 \mathrm{e}(22.5)$ & Kurt \\
\hline $\mathrm{Si}$ & $\mathrm{H}$ & 28 & 1 & 4750.026 & -3.517 & 1.362 & 3.971 & 0.000 & $\mathrm{X}$ & $\hat{n}$ & b & 8 & 5) & Ku \\
\hline $\mathrm{Si}$ & $\mathrm{H}$ & 28 & 1 & 4750.041 & -3.517 & 1.362 & 3.971 & 0.000 & $\mathrm{X}$ & $\mathrm{A}$ & 6 & 8 & $\mathrm{R} 2 \mathrm{e}$ & Kur \\
\hline $\mathrm{Si}$ & $\mathrm{H}$ & 28 & 1 & 4750.119 & -1.745 & 1.405 & 4.015 & 0.000 & $\mathrm{X}$ & A & 3 & 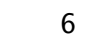 & Q1f(1 & Kur \\
\hline $\mathrm{Ca}$ & $\mathrm{H}$ & 40 & 1 & 4840.955 & -8.362 & 0.141 & 2.701 & 0.000 & $\mathrm{X}$ & $\mathrm{A}$ & $\theta$ & 5 & SR21e (Q16) & B.Plez.pri \\
\hline $\mathrm{Ca}$ & $\mathrm{H}$ & 40 & 1 & 4841.032 & -8.416 & 0.158 & 2.718 & 0.000 & $\mathrm{X}$ & A & $\theta$ & 5 & SR21e(017) & B.Pl \\
\hline $\mathrm{Ca}$ & $\mathrm{H}$ & 40 & 1 & 4748.793 & -6.341 & 0.411 & 3.021 & 0.000 & $\mathrm{X}$ & B & $\theta$ & 5 & 27) & B.Plez.priv \\
\hline $\mathrm{Ca}$ & $\mathrm{H}$ & 40 & 1 & 4753.848 & -9.479 & 0.411 & 3.018 & 0.000 & $\mathrm{X}$ & B & 0 & 3 & $P Q 1$ & B.Pl \\
\hline $\mathrm{Fe}$ & $\mathrm{H}$ & 56 & 1 & 4748.793 & -6.341 & 0.411 & 3.021 & 0.000 & $\mathrm{X}$ & $\mathrm{F}$ & $\theta$ & 5 & $\mathrm{P} 2 \mathrm{e}(027)$ & B.Plez.priv.comm \\
\hline $\mathrm{Fe}$ & $\mathrm{H}$ & 56 & 1 & 848 & -9.4 & 0.411 & 3.018 & 0.000 & $\mathrm{X}$ & $\mathrm{F}$ & 0 & 3 & PQ12 & z.priv.comm \\
\hline $\mathrm{Fe}$ & $\mathrm{H}$ & 56 & 1 & 4754.121 & -6.333 & 0.411 & 3.018 & 0.000 & $\mathrm{X}$ & $\mathrm{F}$ & 0 & 5 & $\mathrm{P} 1 \mathrm{e}(028)$ & B.Plez.priv.comm \\
\hline $\mathrm{Fe}$ & $\mathrm{H}$ & 56 & 1 & 475 & & 77 & 3.4 & 00 & $\mathrm{X}$ & $\mathrm{F}$ & $\theta$ & & 2) & comm \\
\hline $\mathrm{Ti}$ & 0 & 46 & 16 & 4750.674 & 0.046 & 4.105 & 4.694 & 6.521 & $d$ & $\mathrm{~b}$ & 11 & 8 & $Q(184.0)$ & B.Plez.priv.comm \\
\hline $\mathrm{Ti}$ & 0 & 47 & 16 & 4750.672 & -8.717 & 3.193 & 3.782 & 6.831 & $\mathrm{X}$ & A & 13 & 3 & $8.0)$ & B.Plez.priv.comm \\
\hline $\mathrm{Ti}$ & 0 & 48 & 16 & 4750.672 & -8.992 & 3.104 & 3.693 & 7.084 & $\mathrm{E}$ & B & 3 & 4 & Q1(146.0) & B.Plez.priv.comm \\
\hline $\mathrm{Ti}$ & 0 & 49 & 16 & 4750.675 & -4.167 & 4.584 & 5.173 & 7.643 & $\mathrm{E}$ & B & 9 & 11 & P3 (192.0) & B.Plez.priv.comm \\
\hline $\mathrm{Ti}$ & 0 & 50 & 16 & 4750.673 & -5.596 & 2.472 & 3.061 & 7.210 & $\mathrm{E}$ & B & 6 & 7 & $\mathrm{QR} 12(74.0)$ & B.Plez.priv.comm \\
\hline V & 0 & 51 & 16 & 5025.989 & -6.632 & 0.291 & 2.757 & 0.000 & $\mathrm{X}$ & A & 2 & 14 & Q4e (023) & B.Plez.priv.comm \\
\hline$v$ & 0 & 51 & 16 & 5027.160 & -6.601 & 0.294 & 2.760 & 0.000 & $\mathrm{X}$ & A & 2 & 14 & Q4e (024) & B.Plez.priv.comm \\
\hline V & 0 & 51 & 16 & 5054.819 & -5.435 & 0.478 & 2.930 & 0.000 & $\mathrm{X}$ & B & 3 & 12 & Q4e (038) & B.Plez.priv.comm \\
\hline V & 0 & 51 & 16 & 4750.684 & -3.953 & 0.526 & 3.135 & 0.000 & $\mathrm{X}$ & C & 1 & 6 & RQ32e (077) & B.Plez.priv.comm \\
\hline $\mathrm{Zr}$ & 0 & 90 & 16 & 4750.673 & 0.260 & 1.518 & 4.127 & 0.000 & $\mathrm{X}$ & $\mathrm{H}$ & 10 & 11 & R2e (068) & B.Plez.priv.comm \\
\hline $\mathrm{Zr}$ & 0 & 91 & 16 & 4750.672 & -4.525 & 0.174 & 2.783 & 0.000 & $\mathrm{X}$ & G & $\theta$ & 4 & $\mathrm{P} 2 \mathrm{e}(009)$ & B.Plez.priv.comm \\
\hline $\mathrm{Zr}$ & 0 & 92 & 16 & 4750.675 & 0.012 & 1.834 & 4.443 & 0.000 & $\mathrm{X}$ & $\mathrm{H}$ & 7 & 8 & $\mathrm{R} 1 \mathrm{e}(136)$ & B.Plez.priv.comm \\
\hline $\mathrm{Zr}$ & 0 & 94 & 16 & 4750.673 & -2.682 & 0.390 & 2.999 & 0.000 & $\mathrm{X}$ & $\mathrm{H}$ & 1 & 1 & OP12e(Q45) & B.Plez.priv.comm \\
\hline $\mathrm{Zr}$ & 0 & 96 & 16 & 4750.672 & -2.568 & 1.284 & 3.893 & 0.000 & $\mathrm{X}$ & G & 4 & 9 & $\mathrm{R} 2 \mathrm{e}(115)$ & B.Plez.priv.comm \\
\hline
\end{tabular}

Notes. Full table available at CDS. Column headers give field names, where E1, E2, I1, I2, R, S1, and Su are Element_1, Element_2, Isotope_1, Isotope_2, Rad_damp, State_low, and State_up, respectively. See Sect. 4.3 and ReadMe file at CDS for description of fields. 
the values for the fields e_loggf, Rad_damp, Sta_damp, and Vdw_damp are equal to zero, this means that they are not available for the respective transition. Field names starting with $r_{-}$ contain reference codes, that is, labels to be used with the provided BibTeX file (see below). Table 6 does not contain the fields Label_low and Label_up, which are however present for each line in the CDS table. As a single example, the strings given in fields Label_low and Label_up for the CI line listed in Table 6 are 'LS 2s2.2p.3p 1P' and 'LS 2s2.2p.4d 1P*', respectively (where multiple white spaces were collapsed into one).

The molecular data are stored in similar form, with two data fields for both element symbol and isotope information (Element_1, Element_2, Isotope_1, Isotope_2), a subset of the remaining data fields (lambda, loggf, E_low, E_up, Rad_damp), and the following additional data fields:

State_low Lower level electronic state symbol.

State_up Upper level electronic state symbol.

v_low Lower level vibrational quantum number.

v_up Upper level vibrational quantum number.

Branch Label indicating branch - a string of characters in most cases consisting of the branch designation (e.g. P, Q, R),

the spin components $(1,2, \ldots)$, the rotationless parity (e or f) whenever lambda doubling has been computed, and in parentheses the total angular momentum quantum number $(J)$ for the lower level. For further explanations we refer to the references for the sources of molecular data (see Table 4). r_mol Reference code.

We would like to point out that the quantum numbers were not used for the calculation of synthetic spectra within the GES. The data are available in their entirety in a machine-readable form at the CDS. An excerpt is shown in Table 7 for guidance regarding the content of the CDS table.

We strongly encourage users of the Gaia-ESO line list to cite, in addition to this overview article, the individual sources for the atomic and molecular data used in a particular work. It is important that providers of atomic data receive credit for their work by citing the original publications. This is also a prerequisite for the continued funding of this type of research. To facilitate citations of original sources we provide, together with the data tables, a BibTeX file with the relevant entries.

Acknowledgements. We are thankful for the contributions of Enrico Maiorca Matthew P. Ruffoni, and Jennifer Sobeck to the line list work. We thank Robert L. Kurucz for information on his calculations. U.H. and A.J.K. acknowledge support from the Swedish National Space Agency (SNSA/Rymdstyrelsen) K.L. acknowledges funds from the European Research Council (ERC) under the European Union's Horizon 2020 research and innovation programme (Grant agreement No. 852977). Š.M. acknowledges support from the Research Council of Lithuania (LMT) through grant LAT-08/2016. T.M. acknowledges support from the State Research Agency (AEI) of the Spanish Ministry of Science, Innovation and Universities (MCIU) and the European Regional Development Fund (FEDER) under grant AYA2017-88254-P. J.C.P. acknowledges support from the STFC of the UK. T.B. and U.H. were supported by the project grant "The New Milky Way" from the Knut and Alice Wallenberg Foundation. A.R.C. is supported in part by the Australian Research Council through a Discovery Early Career Researcher Award (DE190100656). Parts of this research were supported by the Australian Research Council Centre of Excellence for All Sky Astrophysics in 3 Dimensions (ASTRO 3D), through project number CE170100013. P.J. acknowledges funds from FONDECYT Iniciación Grant number 11170174. R.S. acknowledges support from NCN through grant 2014/15/B/ST9/03981 and from the Polish Ministry of Science and Higher Education. This work has made use of the VALD database, operated at Uppsala University, the Institute of Astronomy RAS in Moscow, and the University of Vienna. Based on data products from observations made with ESO Telescopes at the La Silla Paranal Observatory under programme ID 188.B-3002. These data products have been processed by the Cambridge Astronomy Survey Unit (CASU) at the Institute of Astronomy, University of Cambridge, and by the
FLAMES/UVES reduction team at INAF/Osservatorio Astrofisico di Arcetri. These data have been obtained from the Gaia-ESO Survey Data Archive, prepared and hosted by the Wide Field Astronomy Unit, Institute for Astronomy, University of Edinburgh, which is funded by the UK Science and Technology Facilities Council. This work was partly supported by the European Union FP7 programme through ERC grant number 320360 and by the Leverhulme Trust through grant RPG-2012-541. We acknowledge the support from INAF and Ministero dell' Istruzione, dell' Università' e della Ricerca (MIUR) in the form of the grant "Premiale VLT 2012". The results presented here benefit from discussions held during the Gaia-ESO workshops and conferences supported by the ESF (European Science Foundation) through the GREAT Research Network Programme.

\section{References}

Ahmad, S. A., \& Saksena, G. D. 1981, Spectrochim. Acta, 36, 943

Aldenius, M., Tanner, J. D., Johansson, S., Lundberg, H., \& Ryan, S. G. 2007, A\&A, 461, 767

Aldenius, M., Lundberg, H., \& Blackwell-Whitehead, R. 2009, A\&A, 502, 989

Allard, N. F., Kielkopf, J. F., Cayrel, R., \& van't Veer-Menneret C. 2008, A\&A, 480,581

Anstee, S. D., \& O'Mara, B. J. 1991, MNRAS, 253, 549

Anstee, S. D., \& O'Mara, B. J. 1995, MNRAS, 276, 859

Arnesen, A., Bengtsson, A., Hallin, R., et al. 1977, Phys. Scr., 16, 31, aBH

Asplund, M., Grevesse, N., Sauval, A. J., \& Scott, P. 2009, ARA\&A, 47, 481

Baertschi, P. 1976, Earth Planet. Sci. Lett., 31, 341

Baluja, K. L., \& Zeippen, C. J. 1988, J. Phys. B At. Mol. Phys., 21, 1455

Bard, A., \& Kock, M. 1994, A\&A, 282, 1014

Bard, A., Kock, A., \& Kock, M. 1991, A\&A, 248, 315

Barklem, P. S. 2016, A\&ARv, 24, 9

Barklem, P. S., \& Aspelund-Johansson, J. 2005, A\&A, 435, 373, (BA-J)

Barklem, P. S., \& Collet, R. 2016, A\&A, 588, A96

Barklem, P. S., \& O'Mara, B. J. 1997, MNRAS, 290, 102

Barklem, P. S., \& O'Mara, B. J. 1998, MNRAS, 300, 863

Barklem, P. S., \& O'Mara, B. J. 2000, MNRAS, 311, 535

Barklem, P. S., \& O’Mara, B. J. 2001, J. Phys. B At. Mol. Opt. Phys., 34, 4785

Barklem, P. S., O’Mara, B. J., \& Ross, J. E. 1998, MNRAS, 296, 1057

Barklem, P. S., Piskunov, N., \& O’Mara, B. J. 2000a, A\&A, 363, 1091

Barklem, P. S., Piskunov, N., \& O'Mara, B. J. 2000b, A\&AS, 142, 467

Barklem, P. S., Anstee, S. D., \& O'Mara, B. J. 2015, Astrophysics Source Code Library [record ascl: 1507.007]

Başar, G., Başar, G., Acar, G., Öztürk, I. K., \& Kröger, S. 2003, Phys. Scr., 67, 476

Başar, G., Başar, F. G., Öztürk, İ. K., \& Kröger, S. 2004, Phys. Scr., 69, 189

Bates, D. R., \& Damgaard, A. 1949, Phil. Trans. R. Soc. A Math., Phys. Eng. Sci., 242, 101

Becker, W., Blatt, R., \& Werth, G. 1981, in Precision Measurement and Fundamental Constants, eds. B. N. Taylor \& W. D. Phillips, 99

Bergemann, M., Ruchti, G. R., Serenelli, A., et al. 2014, A\&A, 565, A89

Bergeson, S. D., \& Lawler, J. E. 1993, J. Opt. Soc. Am. B Opt. Phys., 10, 794

Bergström, H., Peng, W. X., \& Persson, A. 1989, Z. Phys. D At. Mol. Clusters, 13, 203

Biémont, E. 1973, Bull. Soc. R. Sci. Liege, 42, 206

Biemont, E., \& Godefroid, M. 1980, A\&A, 84, 361

Biemont, E., Grevesse, N., Hannaford, P., \& Lowe, R. M. 1981, ApJ, 248, 867

Biemont, E., Grevesse, N., Kwiatkovski, M., \& Zimmermann, P. 1984, A\&A, 131,364

Biemont, E., Quinet, P., \& Zeippen, C. J. 1993, A\&AS, 102, 435

Biémont, E., Dutrieux, J., Martin, I., \& Quinet, P. 1998, J. Phys. B At. Mol. Phys., 31, 3321

Biémont, E., Lefèbvre, P., Quinet, P., Svanberg, S., \& Xu, H. L. 2003, Eur. Phys. J. D, 27, 33

Biémont, É., Blagoev, K., Engström, L., et al. 2011, MNRAS, 414, 3350

Bizzarri, A., Huber, M. C. E., Noels, A., et al. 1993, A\&A, 273, 707

Bizzarro, M., Paton, C., Larsen, K., et al. 2011, J. Anal. At. Spectrom., 26, 565

Blackwell, D. E., Ibbetson, P. A., Petford, A. D., \& Shallis, M. J. 1979a, MNRAS, 186,633

Blackwell, D. E., Petford, A. D., \& Shallis, M. J. 1979b, MNRAS, 186, 657

Blackwell, D. E., Petford, A. D., Shallis, M. J., \& Leggett, S. 1982a, MNRAS, 199, 21

Blackwell, D. E., Petford, A. D., Shallis, M. J., \& Simmons, G. J. 1982b, MNRAS, 199, 43

Blackwell, D. E., Petford, A. D., \& Simmons, G. J. 1982c, MNRAS, 201, 595

Blackwell, D. E., Menon, S. L. R., \& Petford, A. D. 1983, MNRAS, 204, 883

Blackwell, D. E., Menon, S. L. R., \& Petford, A. D. 1984, MNRAS, 207, 533 
Blackwell, D. E., Booth, A. J., Haddock, D. J., Petford, A. D., \& Leggett, S. K 1986, MNRAS, 220, 549

Blackwell-Whitehead, R. J., Xu, H. L., Pickering, J. C., Nave, G., \& Lundberg, H. 2005a, MNRAS, 361, 1281

Blackwell-Whitehead, R. J., Pickering, J. C., Pearse, O., \& Nave, G. 2005b, ApJS, 157, 402

Blackwell-Whitehead, R., \& Bergemann, M. 2007, A\&A, 472, L43

Blaise, J., Wyart, J.-F., Tahar Djerad, M., \& Ben Ahmed Z. 1984, Phys. Scr., 29, 119

Blanco, F., Botho, B., \& Campos, J. 1995, Phys. Scr., 52, 628

Blanco-Cuaresma, S., Soubiran, C., Jofré, P., \& Heiter, U. 2014, A\&A, 566, A98

Booth, A. J., Blackwell, D. E., Petford, A. D., \& Shallis, M. J. 1984, MNRAS, 208,147

Bord, D. J., Barisciano, Jr. L. P., \& Cowley, C. R. 1996, MNRAS, 278, 997

Bourauel, C., Rupprecht, W., \& Büttgenbach, S. 1987, Z. Phys. D At. Mol Clusters, 7, 129

Bridges, J. M., \& Kornblith, R. L. 1974, ApJ, 192, 793

Brodzinski, T., Kronfeldt, H.-D., Kropp, J.-R., \& Winkler, R. 1987, Z. Phys. D Atoms Mol. Clusters, 7, 161

Brooke, J. S. A., Bernath, P. F., Schmidt, T. W., \& Bacskay, G. B. 2013, J. Quant. Spectr. Rad. Transf., 124, 11

Brooke, J. S. A., Ram, R. S., Western, C. M., et al. 2014, ApJS, 210, 23

Brown, J. A., Sneden, C., Lambert, D. L., \& Dutchover, Jr. E. 1989, ApJS, 71 , 293

Brueckner, K. A. 1971, ApJ, 169, 621

Butler, K., \& Zeippen, C. J. 1991, J. Phys. IV France, 01, C1

Butler, K., Mendoza, C., \& Zeippen, C. J. 1993, J. Phys. B At. Mol. Phys., 26, 4409

Cardon, B. L., Smith, P. L., Scalo, J. M., Testerman, L., \& Whaling, W. 1982 ApJ, 260, 395

Carlsson, J., Sturesson, L., \& Svanberg, S. 1989, Z. Phys. D Atoms Mol. Clusters, 11,287

Casamiquela, L., Carrera, R., Jordi, C., et al. 2016, MNRAS, 458, 3150

Casimir, H. 1936, On the Interaction Between Atomic Nuclei and Electrons, Archives du musée Teyler (Dordrecht: Springer Netherlands)

Chang, T.-L., \& Li, W.-J. 1990, Chin. Sci. Bull., 35, 290

Chang, T. N., \& Tang, X. 1990, J. Quant. Spectr. Rad. Transf., 43, 207

Chang, T. L., Qian, Q.-Y., Zhao, M.-T., \& Wang, J. 1994, Int. J. Mass Spectrom. Ion Process., 139, 95

Chang, T.-L., Qian, Q.-Y., Zhao, M.-T., Wang, J., \& Lang, Q.-Y. 1995, Int. J. Mass Spectrom. Ion Process., 142, 125

Chang, T.-L., Li, W.-J., Zhao, M.-T., Wang, J., \& Qian, Q.-Y. 2001, Int. J. Mass Spectrom., 207, 13

Chang, T.-L., Zhao, M.-T., Li, W.-J., Wang, J., \& Qian, Q.-Y. 2002, Int. J. Mass Spectrom., 218, 167

Childs, W. J. 1971, Phys. Rev. A, 4, 1767

Childs, W. J., Poulsen, O., Goodman, L. S., \& Crosswhite, H. 1979, Phys. Rev. A, 19,168

Cochrane, E. C. A., Benton, D. M., Forest, D. H., \& Griffith, J. A. R. 1998, J. Phys. B At. Mol. Phys., 31, 2203

Cocke, C. L., Stark, A., \& Evans, J. C. 1973, ApJ, 184, 653

Collins, T. L., Rourke, F. M., \& White, F. A. 1957, Phys. Rev., 105, 196

Condon, E. U., \& Shortley, G. H. 1935, The Theory of Atomic Spectra (University Press, the University of Michigan)

Cooper, J. C., Gibson, N. D., \& Lawler, J. E. 1997, J. Quant. Spectr. Rad. Transf., 58,85

Corliss, C. H., \& Bozman, W. R. 1962, NBS Monograph, Experimental transition probabilities for spectral lines of seventy elements; derived from the NBS Tables of spectral-line intensities, eds. C. H. Corliss, \& W. R. Bozman (US Government Printing Office), 53

Corney, A., \& Williams, O. M. 1972, J. Phys. B At. Mol. Phys., 5, 686

Cowley, C. R. 1973, J. Res. NBS, 77A, 419

Cowley, C. R., \& Corliss, C. H. 1983, MNRAS, 203, 651

Das, D., \& Natarajan, V. 2008, J. Phys. B At. Mol. Phys., 41, 035001

Davidson, M. D., Snoek, L. C., Volten, H., \& Doenszelmann, A. 1992, A\&A, 255,457

Davis, S. J., Wright, J. J., \& Balling, L. C. 1971, Phys. Rev. A, 3, 1220

de Laeter, J. R., \& Bukilic, N. 2005, Int. J. Mass Spectrom., 244, 91

de Laverny, P., Recio-Blanco, A., Worley, C. C., \& Plez, B. 2012, A\&A, 544, A126

Dembczyński, J., Ertmer, W., Johann, U., Penselin, S., \& Stinner, P. 1979, Z. Phys. A Hadrons Nuclei, 291, 207

den Hartog, E. A., Curry, J. J., Wickliffe, M. E., \& Lawler, J. E. 1998, Sol. Phys., 178,239

Den Hartog, E. A., Wickliffe, M. E., \& Lawler, J. E. 2002, ApJS, 141, 255

Den Hartog, E. A., Lawler, J. E., Sneden, C., \& Cowan, J. J. 2003, ApJS, 148 543

Den Hartog, E. A., Herd, M. T., Lawler, J. E., et al. 2005, ApJ, 619, 639, (DHL)
Den Hartog, E. A., Lawler, J. E., Sneden, C., \& Cowan, J. J. 2006, ApJS, 167, 292

Den Hartog, E. A., Lawler, J. E., Sobeck, J. S., Sneden, C., \& Cowan, J. J. 2011, ApJS, 194, 35

Den Hartog, E. A., Ruffoni, M. P., Lawler, J. E., et al. 2014, ApJS, 215, 23

De Silva, G. M., Freeman, K. C., Bland-Hawthorn, J., et al. 2015, MNRAS, 449, 2604

Ding, T., Valkiers, S., Kipphardt, H., et al. 2001, Geochim. Cosmochim. Acta, 65,2433

Doerr, A., \& Kock, M. 1985, J. Quant. Spectr. Rad. Transf., 33, 307

Drozdowski, R., Ignaciuk, M., Kwela, J., \& Heldt, J. 1997, Z. Phys. D Atoms Mol. Clusters, 41, 125

Dulick, M., Bauschlicher, Jr. C. W., Burrows, A., et al. 2003, ApJ, 594, 651

Duquette, D. W., \& Lawler, J. E. 1982, Phys. Rev. A, 26, 330

Duquette, D. W., \& Lawler, J. E. 1985, J. Opt. Soc. Am. B Opt. Phys., 2, 1948

Duquette, D. W., Salih, S., \& Lawler, J. E. 1982a, Phys. Rev. A, 26, 2623

Duquette, D. W., Salih, S., \& Lawler, J. E. 1982b, J. Phys. B At. Mol. Phys., 15, L897

Duquette, D. W., den Hartog, E. A., \& Lawler, J. E. 1986, J. Quant. Spectr. Rad. Transf., 35, 281

El-Kashef, H., \& Ludwig, N. 1992, Phys. B Condens. Matter, 179, 103

Ertmer, W., \& Hofer, B. 1976, Z. Phys. A Hadrons Nuclei, 276, 9

Eugster, O., Tera, F., \& Wasserburg, G. J. 1969, J. Geophys. Res., 74, 3897

Eugster, O., Tera, F., Burnett, D. S., \& Wasserburg, G. J. 1970, J. Geophys. Res., 75,2753

Fedchak, J. A., Den Hartog, E. A., Lawler, J. E., et al. 2000, ApJ, 542, 1109

Fischer, W., Hühnermann, H., \& Kollath, K.-J. 1967, Z. Phys., 200, 158

Fivet, V., Quinet, P., Palmeri, P., et al. 2009, MNRAS, 396, 2124

Flesch, G. D., Capellen, J., \& Svec, H. J. 1966, Adv. Mass Spectrom., 3, 571

Foley, H. M. 1946, Phys. Rev., 69, 616

Froese Fischer, C., \& Tachiev, G. 2012, Multiconfiguration Hartree-Fock and Multiconfiguration Dirac-Hartree-Fock Collection, Version 2, http:// physics.nist.gov/mchf/

Fuhr, J. R., \& Wiese, W. L. 2006, J. Phys. Chem. Ref. Data, 35, 1669

Fuhr, J. R., Martin, G. A., \& Wiese, W. L. 1988, J. Phys. Chem. Ref. Data, 17,4

Furmann, B., Ruczkowski, J., Stefańska, D., Elantkowska, M., \& Dembczyński, J. 2008, J. Phys. B At. Mol. Phys., 41, 215004

Galavis, M. E., Mendoza, C., \& Zeippen, C. J. 1997, A\&AS, 123, 159

Gallagher, A. 1967, Phys. Rev., 157, 24

García, G., \& Campos, J. 1988, J. Quant. Spectr. Rad. Transf., 39, 477

Garz, T. 1973, A\&A, 26, 471

Garz, T., \& Kock, M. 1969, A\&A, 2, 274

Gilmore, G., Randich, S., Asplund, M., et al. 2012, The Messenger, 147, 25

Ginibre, A. 1989, Phys. Scr, 39, 694

Gonfiantini, R., de Bievre, P., Valkiers, S., \& Taylor, P. D. P. 1997, IEEE Trans. Instrum. Measur., 46, 566

Gough, D. S., Hannaford, P., \& Lowe, R. M. 1982, J. Phys. B At. Mol. Phys., 15, L431

Gough, D. S., Hannaford, P., \& Lowe, R. M. 1983, J. Phys. B. At. Mol. Phys., 16, 785

Gramlich, J. W., Machlan, L. A., Barnes, I. L., \& Paulsen, P. J. 1989, J. Res. Natl. Bur. Std., 94,347

Gray, D. F. 2005, The Observation and Analysis of Stellar Photospheres (Cambridge, UK: Cambridge University Press)

Grevesse, N., Blackwell, D. E., \& Petford, A. D. 1989, A\&A, 208, 157

Grevesse, N., Asplund, M., \& Sauval, A. J. 2007, Space Sci. Rev., 130, 105

Grevesse, N., Scott, P., Asplund, M., \& Sauval, A. J. 2015, A\&A, 573, A27

Guiglion, G., de Laverny, P., Recio-Blanco, A., et al. 2016, A\&A, 595, A18

Gustafsson, B., Edvardsson, B., Eriksson, K., et al. 2008, A\&A, 486, 951

Handrich, E., Steudel, A., \& Walther, H. 1969, Phys. Lett. A, 29, 486

Hannaford, P., Lowe, R. M., Grevesse, N., \& Noels, A. 1992, A\&A, 259, 301

Hawkins, K., Jofré, P., Heiter, U., et al. 2016, A\&A, 592, A70

Heise, C., \& Kock, M. 1990, A\&A, 230, 244

Heiter, U., Jofré, P., Gustafsson, B., et al. 2015, A\&A, 582, A49

Hermann, G., Lasnitschka, G., Schwabe, C., \& Spengler, D. 1993, Spectrochim. Acta, 48,1259

Hibbert, A., Biemont, E., Godefroid, M., \& Vaeck, N. 1991, J. Phys. B At. Mol. Phys., 24, 3943

Hibbert, A., Biemont, E., Godefroid, M. \& Vaeck, N. 1993, A\&AS, 99, 179

Hinkle, K., Wallace, L., Valenti, J., \& Harmer, D. 2000, Visible and Near Infrared Atlas of the Arcturus Spectrum 3727-9300 A (San Francisco: ASP)

Hinkle, K. H., Wallace, L., Ram, R. S., et al. 2013, ApJS, 207, 26

Höhle, C., Hühnermann, H., \& Wagner, H. 1982, Z. Phys. A Hadrons Nuclei, 304,279

Howes, L. M., Asplund, M., Keller, S. C., et al. 2016, MNRAS, 460, 884

Huang, M., \& Masuda, A. 1997, Anal. Chem., 69, 1135 
Huang, Y., Barts, S. A., \& Halpern, J. B. 1992, J. Phys. Chem., 96, 425

Huber, K. P., \& Herzberg, G. 1979, Constants of Diatomic Molecules (New York: Van Nostrand Reinhold Company)

Hughes, R. H. 1957, Phys. Rev., 105, 1260

Hühnermann, H., Möller, W., Alkhazov, G., \& Panteleev, V. 1992, Inst. Phys Conf. Ser., 132, 209

Irwin, A. W. 1987, A\&A, 182, 348

Ivarsson, S., Litzén, U., \& Wahlgren, G. M. 2001, Phys. Scr., 64, 455

Ivarsson, S., Andersen, J., Nordström, B., et al. 2003, A\&A, 409, 1141

Jofré, P., Heiter, U., Soubiran, C., et al. 2014, A\&A, 564, A133

Jofré, P., Heiter, U., Soubiran, C., et al. 2015, A\&A, 582, A81

Jofré, P., Heiter, U., Worley, C. C., et al. 2017, A\&A, 601, A38

Jofré, P., Heiter, U., \& Soubiran, C. 2019, ARA\&A, 57, 571

Johann, U., Dembczyński, J., \& Ertmer, W. 1981, Z. Phys. A Hadrons Nuclei, 303,7

Johansson, S., Litzén, U., Lundberg, H., \& Zhang, Z. 2003, ApJ, 584, L107

Kerkeni, B., Barklem, P. S., Spielfiedel, A., \& Feautrier, N. 2004, J. Phys. B At. Mol. Opt. Phys., 37, 677

Kerkhoff, H., Schmidt, M., \& Zimmermann, P. 1980, Z. Phys. A Hadrons Nuclei, 298, 249

Kock, M., \& Richter, J. 1968, ZAp, 69, 180

Kock, M., Kroll, S., \& Schnehage, S. 1984, Phys. Scr., 8, 84

Koczorowski, W., Stachowska, E., Furmann, B., et al. 2005, Spectrochim. Acta, 60,447

Kopfermann, H. 1958, Nuclear Moments, Pure and Applied Physics, v.2 (Boston: Academic Press)

Kramida, A., Ralchenko, Y., Reader, J., \& NIST ASD Team 2018, NIST Atomic Spectra Database (ver. 5.6), Available: https://physics.nist.gov/asd [Oct 09 2018], National Institute of Standards and Technology, Gaithersburg, MD

Kroll, S., \& Kock, M. 1987, A\&AS, 67, 225

Kułaga-Egger, D., \& Migdałek, J. 2009, J. Phys. B At. Mol. Phys., 42, 185002

Kumar, A., Hsiao, C.-C., Hung, W.-C., \& Lee, Y.-P. 1998, J. Chem. Phys., 109, 3824

Kurucz, R. L. 1970, SAO Special Report, 309

Kurucz, R. L. 1981, SAO Special Report, 390

Kurucz, R. 1993, CDROM 18, SAO, Cambridge

Kurucz, R. L. 1999, Robert L. Kurucz on-line database of observed and predicted atomic transitions, http://kurucz . harvard.edu/atoms/2001/, 2401/

Kurucz, R. L. 2003, Robert L. Kurucz on-line database of observed and predicted atomic transitions, http://kurucz . harvard.edu/atoms/2801/

Kurucz, R. L. 2004, Robert L. Kurucz on-line database of observed and predicted atomic transitions, http://kurucz . harvard.edu/atoms/1600/

Kurucz, R. L. 2006, Robert L. Kurucz on-line database of observed and predicted atomic transitions, http://kurucz . harvard.edu/atoms/2701/, 3900/

Kurucz, R. L. 2007, Robert L. Kurucz on-line database of observed and predicted atomic transitions, http://kurucz.harvard.edu/atoms/1400/, 2000/, $2500 /, 2600 /$

Kurucz, R. L. 2008, Robert L. Kurucz on-line database of observed and predicted atomic transitions, http: //kurucz.harvard.edu/atoms/2700/, 2800/

Kurucz, R. L. 2009, Robert L. Kurucz on-line database of observed and predicted atomic transitions, http://kurucz .harvard.edu/atoms/2100/, 2101/, $2300 /, 2400 /$

Kurucz, R. L. 2010a, Robert L. Kurucz on-line database of molecular line lists, $\mathrm{SiH}$ A-X transitions, $(\mathrm{KSiH})$

Kurucz, R. L. 2010b, Robert L. Kurucz on-line database of observed and predicted atomic transitions, http://kurucz.harvard.edu/atoms/0600/, 2001/, 2200/, 2201/, 2301/, 2400/, 2401/, 2601/

Kurucz, R. L. 2011, Robert L. Kurucz on-line database of observed and predicted atomic transitions, http://kurucz . harvard. edu/atoms/3901/

Kurucz, R. L. 2012, Robert L. Kurucz on-line database of observed and predicted atomic transitions, http://kurucz.harvard.edu/atoms/1501/, 1900, 1901

Kurucz, R. L. 2013, Robert L. Kurucz on-line database of observed and predicted atomic transitions, http://kurucz . harvard.edu/atoms/2601/

Kurucz, R. L. 2014, Model Atmosphere Codes: ATLAS12 and ATLAS9, eds. E. Niemczura, B. Smalley, \& W. Pych, 39

Kurucz, R., \& Bell, B. 1995, Atomic Line Data, Kurucz CD-ROM No. 23 (Cambridge, MA: Smithsonian Astrophysical Observatory)

Kurucz, R. L., \& Peytremann, E. 1975, SAO Special Report, 362, 1

Kurucz, R. L., Furenlid, I., Brault, J., \& Testerman, L. 1984, Solar flux atlas from 296 to $1300 \mathrm{~nm}$

Kurz, N., Dietrich, M. R., Shu, G., et al. 2008, Phys. Rev. A, 77, 060501

Kwiatkowski, M., Zimmermann, P., Biemont, E., \& Grevesse, N. 1984, A\&A, 135,59

Langlois, E., \& Gagné, J.-M. 1994, J. Opt. Soc. Am. B Opt. Phys., 11, 552

Lanzafame, A. C., Frasca, A., Damiani, F., et al. 2015, A\&A, 576, A80

Lawler, J. E., \& Dakin, J. T. 1989, J. Opt. Soc. Am. B Opt. Phys., 6, 1457
Lawler, J. E., Whaling, W., \& Grevesse, N. 1990, Nature, 346, 635

Lawler, J. E., Bonvallet, G., \& Sneden, C. 2001a, ApJ, 556, 452

Lawler, J. E., Wickliffe, M. E., Cowley, C. R., \& Sneden, C. 2001b, ApJS, 137, 341

Lawler, J. E., Wickliffe, M. E., den Hartog, E. A., \& Sneden, C. 2001c, ApJ, 563, 1075

Lawler, J. E., Den Hartog, E. A., Sneden, C., \& Cowan, J. J. 2006, ApJS, 162 227

Lawler, J. E., den Hartog, E. A., Labby, Z. E., et al. 2007, ApJS, 169, 120

Lawler, J. E., Hartog, E. A., Sneden, C., \& Cowan, J. J. 2008a, Can. J. Phys., 86, 1033

Lawler, J. E., Sneden, C., Cowan, J. J., et al. 2008b, ApJS, 178, 71

Lawler, J. E., Sneden, C., Cowan, J. J., Ivans, I. I., \& Den Hartog, E. A. 2009, ApJS, 182, 51

Lawler, J. E., Guzman, A., Wood, M. P., Sneden, C., \& Cowan, J. J. 2013, ApJS, 205,11

Lawrence, G. M. 1967, ApJ, 148, 261

Lefèbvre, P.-H., Garnir, H.-P., \& Biémont, E. 2002, Phys. Scr, 66, 363

Lefèbvre, P.-H., Garnir, H.-P., \& Biémont, E. 2003, A\&A, 404, 1153

Leipziger, F. D. 1963, Appl. Spectr., 17, 158

Leland, W. T. 1950, Phys. Rev., 77, 634

Lennard, W. N., Whaling, W., Scalo, J. M., \& Testerman, L. 1975, ApJ, 197, 517

Le Roy R. J. 2017a, J. Quant. Spectr. Rad. Transf., 186, 158

Le Roy R. J. 2017b, J. Quant. Spectr. Rad. Transf., 186, 167

Li, W.-J., Jin, D., \& Chang, T.-L. 1988, Chin. Sci. Bull., 33, 1610

Li, G.-W., Zhang, X.-M., Lu, F.-Q., Peng, X.-J., \& Yang, F.-J. 2001, Jpn. J. Appl. Phys., 40, 2508

Li, R., Chatelain, R., Holt, R. A., et al. 2007a, Phy. Scr., 76, 577

Li, R., Rehse, S. J., Scholl, T. J., et al. 2007b, Can. J. Phys., 85, 1343

Lim, C., Nomaru, K., \& Izawa, Y. 1998, Jpn. J. Appl. Phys., 37, 5049

Lind, K., Koposov, S. E., Battistini, C., et al. 2015, A\&A, 575, L12

Lind, K., Amarsi, A. M., Asplund, M., et al. 2017, MNRAS, 468, 4311

Lindgård, A., \& Nielson, S. E. 1977, At. Data Nucl. Data Tables, 19, 533

Lindholm, E. 1946, Arkiv. Mat. Astron. Fys., 32, A:17

Ljung, G., Nilsson, H., Asplund, M., \& Johansson, S. 2006, A\&A, 456, 1181

Lotrian, J., \& Guern, Y. 1982, J. Phys. B At. Mol. Phys., 15, 69

Luc, P., \& Gerstenkorn, S. 1972, A\&A, 18, 209

Lundqvist, M., Nilsson, H., Wahlgren, G. M., et al. 2006, A\&A, 450, 407

Luo, Y. 2007, Comprehensive Handbook of Chemical Bond Energies (Boca Raton: CRC Press)

Luo, D., \& Pradhan, A. K. 1989, J. Phys. B At. Mol. Phys., 22, 3377

Ma, H. L., \& Yang, F. J. 2004, At. Data Nuclear Data Tables, 86, 3

Magrini, L., Randich, S., Donati, P., et al. 2015, A\&A, 580, A85

Margenau, H. 1939, Rev. Mod. Phys., 11, 1

Marsden, G. C., den Hartog, E. A., Lawler, J. E., Dakin, J. T., \& Roberts, V. D. 1988, J. Opt. Soc. Am. B Opt. Phys., 5, 606

Martin, G., Fuhr, J., \& Wiese, W. 1988, J. Phys. Chem. Ref. Data Suppl., 17

Martin, W. C., Musgrove, A., Kotochigova, S., \& Sansonetti, J. E. 2011 Ground Levels and Ionization Energies for the Neutral Atoms (version 1.4.1) Available: http://physics .nist . gov/IonEnergy [06-Jul-2012 13:25:00 EDT]. National Institute of Standards and Technology, Gaithersburg, MD

Mashonkina, L., Korn, A. J., \& Przybilla, N. 2007, A\&A, 461, 261

Masseron, T., Plez, B., Van Eck, S., et al. 2014, A\&A, 571, A47

Masterman, D., Rosner, S. D., Scholl, T. J., Sharikova, A., \& Holt, R. 2003, Can. J. Phys., 81, 1389

Matheron, P., Escarguel, A., Redon, R., Lesage, A., \& Richou, J. 2001, J. Quant. Spectr. Rad. Transf., 69, 535

May, M., Richter, J., \& Wichelmann, J. 1974, A\&AS, 18, 405

Mayer, A. J., \& Wieser, M. E. 2014, J. Anal. At. Spectrom., 29, 85

McAlexander, W. I., Abraham, E. R. I., \& Hulet, R. G. 1996, Phys. Rev. A, 54

Meggers, W. F., Corliss, C. H., \& Scribner, B. F. 1975, Tables of spectral-line intensities. Part I, II_- arranged by elements, eds. Meggers, W. F., Corliss, C. H., \& Scribner, B. F.

Meija, J., Coplen, T. B., Berglund, M., et al. 2016, Pure \& Appl. Chem., 88, 293

Meléndez, J., \& Barbuy, B. 2009, A\&A, 497, 611

Mendoza, C., Eissner, W., LeDourneuf, M., \& Zeippen, C. J. 1995, J. Phys. B At. Mol. Phys., 28, 3485

Mikolaitis, Š., Hill, V., Recio-Blanco, A., et al. 2014, A\&A, 572, A33

Miles, B. M., \& Wiese, W. L. 1969, At. Data, 1, 1

Miller, M. H., Roig, R. A., \& Bengtson, R. D. 1971, Phys. Rev. A, 4, 1709

Möller, W., Hühnermann, H., Alkhazov, G., \& Panteleev, V. 1993, Phys. Rev. Lett., 70, 541

Moore, L. J., \& Machlan, L. A. 1972, Anal. Chem., 44, 2291

Moore, L. J., Murphy, T. J., Barnes, I. L., \& Paulsen, P. J. 1982, J. Res. Natl. Bur. Std., 87, 1

Nahar, S. N. 1993, Phys. Scr., 48, 297

Nakai, H., Jin, W.-G., Kawamura, M., et al. 2007, Jpn. J. Appl. Phys., 46, 815 
Nakhate, S. G., Afzal, S. M., \& Ahmad, S. A. 1997, Z. Phys. D Atoms Mol. Clusters, 42, 71

Naulin, C., Hedgecock, I. M., \& Costes, M. 1997, Chem. Phys. Lett., 266, 335

Nilsson, H., \& Ivarsson, S. 2008, A\&A, 492, 609

Nilsson, H., Zhang, Z. G., Lundberg, H., Johansson, S., \& Nordström, B. 2002, A\&A, 382, 368

Nilsson, H., Hartman, H., Engström, L., et al. 2010, A\&A, 511, A16

Nitz, D. E., Bergeson, S. D., \& Lawler, J. E. 1995, J. Opt. Soc. Am. B Opt. Phys. 12,377

Nitz, D. E., Kunau, A. E., Wilson, K. L., \& Lentz, L. R. 1999, ApJS, 122, 557

Nitz, D. E., Wickliffe, M. E., \& Lawler, J. E. 1998, ApJS, 117, 313

Nomura, M., Kogure, K., \& Okamoto, M. 1983, Int. J. Mass Spectrom. Ion Process., 50, 219

Nordlander, T., \& Lind, K. 2017, A\&A, 607, A75

O’Brian, T. R., \& Lawler, J. E. 1991, Phys. Rev. A, 44, 7134

O’Brian, T. R., Wickliffe, M. E., Lawler, J. E., Whaling, W., \& Brault, J. W. 1991 J. Opt. Soc. Am. B Opt. Phys., 8, 1185

Öztürk, I. K., Acar, F. G., Başar, G., Başar, G., \& Krüger, S. 2007, Phys. Scr., 75,624

Palmeri, P., Biemont, E., Aboussaid, A., \& Godefroid, M. 1995, J. Phys. B At. Mol. Phys., 28, 3741

Palmeri, P., Quinet, P., Wyart, J., \& Biémont, E. 2000, Phys. Scr., 61, 323

Pancino, E., Lardo, C., Altavilla, G., et al. 2017, A\&A, 598, A5

Pehlivan Rhodin, A., Belmonte, M. T., Engström, L., et al. 2017a, MNRAS, 472, 3337

Pehlivan Rhodin, A., Hartman, H., Nilsson, H., \& Jönsson, P. 2017b, A\&A, 598 A 102

Penkin, N. P., \& Komarovskii, V. A. 1976, J. Quant. Spectr. Rad. Transf., 16, 217

Penkin, N. P., Gorshkov, V. N., \& Komarovskii, V. A. 1984, Opt. Spectr., 57, 488

Peterson, R. C., \& Kurucz, R. L. 2015, ApJS, 216, 1

Peterson, R. C., Kurucz, R. L., \& Ayres, T. R. 2017, ApJS, 229, 23

Pickering, J. C. 1996, ApJS, 107, 811

Pickering, J. C., Thorne, A. P., \& Perez, R. 2001, ApJS, 132, 403

Pinnington, E. H., Ji, Q., Guo, B., et al. 1993, Can. J. Phys., 71, 470

Piskunov, N., \& Valenti, J. A. 2017, A\&A, 597, A16

Piskunov, N. E., Kupka, F., Ryabchikova, T. A., Weiss, W. W., \& Jeffery, C. S. 1995, A\&AS, 112, 525

Ponzevera, E., Quétel, C. R., Berglund, M., et al. 2006, J. Am. Soc. Mass Spectrom., 17, 1413

Prochaska, J. X., Naumov, S. O., Carney, B. W., McWilliam, A., \& Wolfe, A. M 2000, AJ, 120, 2513

Prša, A., Harmanec, P., Torres, G., et al. 2016, AJ, 152, 41

Qi, H. P., Taylor, P. D. P., Berglund, M., \& de Bievre P. 1997, Int. J. Mass Spectrom. Ion Process., 171, 263

Quinet, P., Palmeri, P., \& Biemont, E. 1999a, J. Quant. Spectr. Rad. Transf., 62 625

Quinet, P., Palmeri, P., Biémont, E., et al. 1999b, MNRAS, 307, 934

Raassen, A. J. J., \& Uylings, P. H. M. 1998, A\&A, 340, 300

Raassen, A. J. J., Pickering, J. C., \& Uylings, P. H. M. 1998, A\&AS, 130, 541

Radziemski, L. J., Engleman, Jr. R., \& Brault, J. W. 1995, Phys. Rev. A, 52, 4462

Ralchenko, Y., Kramida, A., Reader, J., \& NIST ASD Team. 2010, NIST Atomi Spectra Database (ver. 4.0.0)

Ralchenko, Y., Kramida, A. E., Reader, J., \& NIST ASD Team. 2011, NIST Atomic Spectra Database (ver. 4.1.0), http://physics.nist.gov/asd National Institute of Standards and Technology, Gaithersburg, MD

Ram, R. S., Brooke, J. S. A., Bernath, P. F., Sneden, C., \& Lucatello, S. 2014 ApJS, 211, 5

Randich, S., Gilmore, G., \& Gaia-ESO Consortium. 2013, The Messenger, 154, 47

Recio-Blanco, A., de Laverny, P., Kordopatis, G., et al. 2014, A\&A, 567, A5

Rehse, S. J., Li, R., Scholl, T. J., et al. 2006, Can. J. Phys., 84, 723

Richter, J., \& Wulff, P. 1970, A\&A, 9, 37

Rivest, R. C., Izawa, M. R., Rosner, S. D., et al. 2002, Can. J. Phys., 80, 557

Rosner, S. D., Masterman, D., Scholl, T. U., \& Holt, R. A. 2005, Can. J. Phys., 83,841

Rudolph, J., \& Helbig, V. 1982, J. Phys. B At. Mol. Phys., 15, L599

Ruffoni, M. P., Den Hartog, E. A., Lawler, J. E., et al. 2014, MNRAS, 441, 3127

Ryabchikova, T. A., Hill, G. M., Landstreet, J. D., Piskunov, N., \& Sigut, T. A. A 1994, MNRAS, 267, 697

Ryabchikova, T., Ryabtsev, A., Kochukhov, O., \& Bagnulo, S. 2006, A\&A, 456, 329

Ryabchikova, T., Piskunov, N., Kurucz, R. L., et al. 2015, Phys. Scr., 90, 054005

Salih, S., \& Lawler, J. E. 1985, J. Opt. Soc. Am. B Opt. Phys., 2, 422

Salih, S., \& Lawler, J. E. 1990, A\&A, 239, 407

Salih, S., Duquette, D. W., \& Lawler, J. E. 1983, Phys. Rev. A, 27, 1193

Sansonetti, C. J., Richou, B., Engleman, Jr. R., \& Radziemski, L. J. 1995 Phys. Rev. A, 52, 2682
Saraph, H., \& Storey, P. 2012, TOPbase, available at http://cdsweb. u-strasbg.fr/topbase/topbase.html

Schnabel, R., Kock, M., \& Holweger, H. 1999, A\&A, 342, 610

Schnabel, R., Schultz-Johanning, M., \& Kock, M. 2004, A\&A, 414, 1169

Schulz-Gulde, E. 1969, J. Quant. Spectr. Rad. Transf., 9, 13

Scott, P., Grevesse, N., Asplund, M., et al. 2015a, A\&A, 573, A25

Scott, P., Asplund, M., Grevesse, N., Bergemann, M., \& Sauval, A. J. 2015b, A\&A, 573, A26

Seaton, M. J., Yan, Y., Mihalas, D., \& Pradhan, A. K. 1994, MNRAS, 266, 805

Sen, A., \& Childs, W. J. 1987, Phys. Rev. A, 36, 1983

Shayesteh, A., Henderson, R. D. E., Roy, R. J. L., \& Bernath, P. F. 2007, J. Phys Chem. A, 111, 12495

Shields, W. R., Goldich, S. S., Garner, E. L., \& Murphy, T. J. 1965, J. Geophys. Res., 70, 479

Shields, W. R., Murphy, T. J., Catanzaro, E. J., \& Garner, E. L. 1966, J. Res. Natl. Bur. Std., 70

Shima, M., \& Torigoye, N. 1993, Int. J. Mass Spectrom. Ion Process., 123, 29

Shore, B. W. 1967, J. Opt. Soc. Am. 1917-1983, 57, 881

Sigut, T. A. A., \& Landstreet, J. D. 1990, MNRAS, 247, 611

Silverans, R. E., Borghs, G., de Bisschop, P., \& van Hove M. 1986, Phys. Rev. A, 33,2117

Smiljanic, R., Korn, A. J., Bergemann, M., et al. 2014, A\&A, 570, A122

Smith, G. 1981, A\&A, 103, 351

Smith, G. 1988, J. Phys. B At. Mol. Phys., 21, 2827

Smith, G., \& Raggett, D. S. J. 1981, J. Phys. B At. Mol. Phys., 14, 4015

Smith, P. L., \& Kuehne, M. 1978, R. Soc. London Proc. Ser. A, 362, 263

Sneden, C., Lawler, J. E., Cowan, J. J., Ivans, I. I., \& Den Hartog E. A. 2009, ApJS, 182, 80

Sneden, C., Lucatello, S., Ram, R. S., Brooke, J. S. A., \& Bernath, P. 2014, ApJS, 214, 26

Sneden, C. A. 1973, PhD thesis, The University of Texas at Austin, USA

Sobeck, J. S., Lawler, J. E., \& Sneden, C. 2007, ApJ, 667, 1267

Stehlé, C., \& Hutcheon, R. 1999, A\&AS, 140, 93

Stetson, P. B., \& Pancino, E. 2008, PASP, 120, 1332

Stetson, P. B., \& Pancino, E. 2010, Astrophysics Source Code Library [record ascl : 1011.002$]$

Storey, P. J., \& Zeippen, C. J. 2000, MNRAS, 312, 813

Sur, C., Chaudhuri, R. K., Das, B. P., \& Mukherjee, D. 2005, J. Phys. B At. Mol. Phys., 38, 4185

Tachiev, G., \& Froese Fischer C. 2001, Can. J. Phys., 79, 955

Tarroni, R., Palmieri, P., Mitrushenkov, A., Tosi, P., \& Bassi, D. 1997, J. Chem. Phys., 106, 10265

Tautvaišienè, G., Drazdauskas, A., Mikolaitis, Š., et al. 2015, A\&A, 573, A55

Taylor, P. D. P., Maeck, R., \& de Bièvre P. 1992, Int. J. Mass Spectrom. Ion Process., 121, 111

Tennyson, J., Yurchenko, S. N., Al-Refaie, A. F., et al. 2020, J. Quant. Spectr. Rad. Transf., 255, 107228

Theodosiou, C. E. 1989, Phys. Rev. A, 39, 4880

Unkel, P., Buch, P., Dembczyński, J., Ertmer, W., \& Johann, U. 1989, Z. Phys. D Atoms Mol. Clusters, 11, 259

Unsöld, A. 1955, Physik der Sternatmosphären, mit besonderer Berücksichtigung der Sonne. (Berlin: Springer)

Vaeck, N., Godefroid, M., \& Hansen, J. E. 1988, Phys. Rev. A, 38, 2830

Valenti, J. A., \& Piskunov, N. 1996, A\&AS, 118, 595

Van Hove, M., Borghs, G., DeBisschop, P., \& Silverans, R. E. 1982, J. Phys. B At. Mol. Phys., 15, 1805

Vidal, C. R., Cooper, J., \& Smith, E. W. 1973, ApJS, 25, 37

Villemoes, P., Arnesen, A., Heijkenskjöld, F., Kastberg, A., \& Wännström, A 1992, Phys. Lett. A, 162, 178

Villemoes, P., Arnesen, A., Heijkenskjold, F., \& Wannstrom, A. 1993, J. Phys. B At. Mol. Phys., 26, 4289

Volz, U., \& Schmoranzer, H. 1996, Phys. Scr. T, 65, 48

Volz, U., Majerus, M., Liebel, H., Schmitt, A., \& Schmoranzer, H. 1996, Phys. Rev. Lett., 76, 2862

von der Goltz, D., Hansen, W., \& Richter, J. 1984, Phys. Scr., 30, 244

Wagner, S. 1955, Z. Phys., 141, 122

Wahlgren, G. M. 2010, EAS Publ. Ser., 43, 91

Ward, L., Vogel, O., Arnesen, A., Hallin, R., \& Wännström, A. 1985, Phys. Scr. 31, 161

Warner, B. 1967, MNRAS, 136, 381

Warner, B. 1968, MNRAS, 139, 115

Wendt, K., Ahmad, S. A., Buchinger, F., et al. 1984, Z. Phys. A Hadrons Nuclei, 318,125

Werij, H. G. C., Greene, C. H., Theodosiou, C. E., \& Gallagher, A. 1992, Phys. Rev. A, 46, 1248

Western, C. M. 2017, J. Quant. Spectr. Rad. Transf., 186, 221

Whaling, W., \& Brault, J. W. 1988, Phys. Scr., 38, 707 
Whaling, W., Hannaford, P., Lowe, R. M., Biemont, E., \& Grevesse, N. 1985, A\&A, 153, 109

White, F. A., Collins, T. L., \& Rourke, F. M. 1956, Phys. Rev., 101, 1786

Wickliffe, M. E., \& Lawler, J. E. 1997a, ApJS, 110, 163

Wickliffe, M. E., \& Lawler, J. E. 1997b, J. Opt. Soc. Am. B Opt. Phys., 14, 737

Wickliffe, M. E., Salih, S., \& Lawler, J. E. 1994, J. Quant. Spectr. Rad. Transf., 51,545

Wickliffe, M. E., Lawler, J. E., \& Nave, G. 2000, J. Quant. Spectr. Rad. Transf., 66,363

Wiese, W. L., \& Fuhr, J. R. 2007, J. Phys. Chem. Ref. Data, 36, 1287

Wiese, W. L. \& Fuhr, J. R. 2009, J. Phys. Chem. Ref. Data, 38, 565

Wiese, W. L., Smith, M. W., \& Miles, B. M. 1969, Atomic transition probabilities. Vol. 2: Sodium through Calcium. A critical data compilation, eds. Wiese, W. L., Smith, M. W., \& Miles, B. M. (US Government Printing Office)

Williams, A. A., Evans, N. W., Molloy, M., et al. 2016, ApJ, 824, L29

Wolnik, S. J., Berthel, R. O., Larson, G. S., Carnevale, E. H., \& Wares, G. W. 1968, Phys. Fluids, 11, 1002

Wolnik, S. J., Berthel, R. O., \& Wares, G. W. 1970, ApJ, 162, 1037

Wolnik, S. J., Berthel, R. O., \& Wares, G. W. 1971, ApJ, 166, L31

Wood, M. P., Lawler, J. E., Sneden, C., \& Cowan, J. J. 2013, ApJS, 208, 27

Wood, M. P., Lawler, J. E., Sneden, C., \& Cowan, J. J. 2014, ApJS, 211, 20

Worley, C. C., Jofré, P., Rendle, B., et al. 2020, A\&A, 643, A83

Xu, H. L., Svanberg, S., Cowan, R. D., et al. 2003a, MNRAS, 346, 433

Xu, H. L., Svanberg, S., Quinet, P., Garnir, H. P., \& Biémont, E. 2003b, J. Phys. B At. Mol. Phys., 36, 4773

Xu, H., Jiang, Z., Zhang, Z., et al. 2003c, J. Phys. B At. Mol. Phys., 36, 1771

Yan, Z.-C., \& Drake, G. W. F. 1995, Phys. Rev. A, 52, 4316

Yan, Z.-C., Tambasco, M., \& Drake, G. W. F. 1998, Phys. Rev. A, 57, 1652

Zatsarinny, O., \& Bartschat, K. 2006, J. Phys. B At. Mol. Phys., 39, 2861

Zeiske, W., Meisel, G., Gebauer, H., Hofer, B., \& Ertmer, W. 1976, Phys. Lett. A, 55,405

Zhao, M., Zhou, T., Wang, J., Lu, H., \& Xiang, F. 2005, Int. J. Mass Spectr., 245, 36

Zhiguo, Z., Zhongshan, L., \& Zhankui, J. 1999, Eur. Phys. J. D, 7, 499

Zhiguo, Z., Li, Z. S., Lundberg, H., et al. 2000, J. Phys. B At. Mol. Phys., 33, 521

1 Observational Astrophysics, Department of Physics and Astronomy, Uppsala University, Box 516, 75120 Uppsala, Sweden

e-mail: ulrike.heiter@physics.uu.se

2 Department of Astronomy, Stockholm University, AlbaNova, Roslagstullbacken 21, 10691 Stockholm, Sweden

${ }^{3}$ Max-Planck Institut für Astronomie (MPIA), Königstuhl 17, 69117 Heidelberg, Germany

${ }^{4}$ Research School of Astronomy \& Astrophysics, Australian National University, Cotter Road, Weston Creek, ACT 2611, Australia
${ }^{5}$ Institute of Theoretical Physics and Astronomy, Vilnius University, Saulètekio av. 3, 10257 Vilnius, Lithuania

${ }^{6}$ Instituto de Astrofísica de Canarias, 38205 La Laguna, Tenerife, Spain

7 Departamento de Astrofísica, Universidad de La Laguna, 38206 La Laguna, Tenerife, Spain

8 Université Côte d'Azur, Observatoire de la Côte d'Azur, CNRS, Laboratoire Lagrange, Blvd de l'Observatoire, 06304 Nice, France

9 INAF-Osservatorio Astrofisico di Arcetri, Largo E. Fermi 5, 50125 Florence, Italy

${ }^{10}$ Materials Science and Applied Mathematics, Malmö University, 205 06 Malmö, Sweden

${ }^{11}$ Lund Observatory, Department of Astronomy and Theoretical Physics, Lund University, Box 43, 22100 Lund, Sweden

12 Blackett Laboratory, Imperial College London, London SW7 2BW, UK

13 Instituto de Física y Astronomía, Facultad de Ciencias, Universidad de Valparaíso, Av. Gran Bretaña 1111, 5030 Casilla, Valparaíso, Chile

14 Núcleo Milenio de Formación Planetaria - NPF, Universidad de Valparaíso, Av. Gran Bretaña 1111, Valparaíso, Chile

15 Institute of Astronomy, University of Cambridge, Madingley Road, Cambridge CB3 0HA, UK

16 School of Physics \& Astronomy, Monash University, Wellington Rd, Clayton 3800, Victoria, Australia

${ }^{17}$ Center of Excellence for Astrophysics in Three Dimensions (ASTRO-3D), Australia

${ }_{18}$ Núcleo de Astronomía, Facultad de Ingeniería, Universidad Diego Portales, Av. Ejército 441, Santiago, Chile

${ }^{19}$ Space Science Data Center - Agenzia Spaziale Italiana, Via del Politecnico, s.n.c., 00133 Roma, Italy

20 INAF - Osservatorio Astronomico di Palermo, Piazza del Parlamento 1, 90134 Palermo, Italy

${ }^{21}$ Dipartimento di Fisica e Astronomia, Sezione Astrofisica, Universitá di Catania, Via S. Sofia 78, 95123 Catania, Italy

${ }^{22}$ Laboratoire d'astrophysique, Ecole Polytechnique Fédérale de Lausanne (EPFL), Observatoire de Sauverny, 1290 Versoix, Switzerland

23 Departamento de Ciencias Fisicas, Universidad Andres Bello, Fernandez Concha 700, Las Condes, Santiago, Chile

${ }^{24}$ Nicolaus Copernicus Astronomical Center, Polish Academy of Sciences, ul. Bartycka 18, 00-716 Warsaw, Poland

25 INAF - Padova Observatory, Vicolo dell'Osservatorio 5, 35122 Padova, Italy 


\section{Appendix A: Auxiliary tables}

This appendix provides five tables with auxiliary data referred to in Sects. 2.1, 3, and 4.2.

Table A.1. Transitions for which multiple fine-structure components were merged into one line in the preselected line list.

\begin{tabular}{|c|c|c|c|c|c|c|}
\hline \multirow[t]{2}{*}{ Species } & \multirow{2}{*}{$\begin{array}{l}\text { Wavelength } \\
{[\AA]}\end{array}$} & \multirow[t]{2}{*}{$N$} & \multirow{2}{*}{$J_{\text {low }}$} & \multirow{2}{*}{$\begin{array}{l}\text { Actual } \\
J_{\text {upp }}\end{array}$} & \multicolumn{2}{|c|}{ GES line list } \\
\hline & & & & & $J_{\text {low }}$ & $J_{\text {upp }}$ \\
\hline O I & 6158.1858 & 3 & 3.0 & $2.0,3.0,4.0$ & 3.0 & 10.0 \\
\hline $\mathrm{NaI}$ & 4982.814 & 2 & 1.5 & $1.5,2.5$ & 1.5 & 4.5 \\
\hline $\mathrm{Na} I$ & 5688.205 & 2 & 1.5 & $1.5,2.5$ & 1.5 & 4.5 \\
\hline $\mathrm{Mg}$ I & 8717.825 & 3 & 2.0 & $1.0,2.0,3.0$ & 2.0 & 7.0 \\
\hline \multirow[t]{3}{*}{$\mathrm{Mg}$ I } & 8736.019 & 6 & 1.0 & 2.0 & 7.0 & 10.0 \\
\hline & & & 2.0 & $2.0,3.0$ & & \\
\hline & & & 3.0 & $2.0,3.0,4.0$ & & \\
\hline $\mathrm{Al} \mathrm{I}$ & 8773.896 & 2 & 2.5 & $2.5,3.5$ & 2.5 & 6.5 \\
\hline \multirow[t]{2}{*}{ Ca II } & 5339.188 & 3 & 2.5 & 3.5 & 6.5 & 8.5 \\
\hline & & & 3.5 & $3.5,4.5$ & & \\
\hline \multirow[t]{2}{*}{ Ca II } & 6456.875 & 3 & 2.5 & 3.5 & 6.5 & 8.5 \\
\hline & & & 3.5 & $3.5,4.5$ & & \\
\hline Ca II & 8927.356 & 2 & 2.5 & $2.5,3.5$ & 2.5 & 6.5 \\
\hline
\end{tabular}

Notes. $N$ - Number of components merged. The following columns give the $J$ values of the lower and upper levels of the original transitions, and the $J$ values quoted in the line list. The latter were obtained by summing up the $J$ values occurring in the respective level for the merged transitions (ignoring repetitions), and adding 1 in the case of integer $J$ values and 0.5 in the case of half-integer $J$ values.

Table A.2. Polynomial coefficients for partition functions $(Q)$ for molecules.

\begin{tabular}{|c|c|c|c|c|c|c|}
\hline Mol. & $a_{0}$ & $a_{1}$ & $a_{2}$ & $a_{3}$ & $a_{4}$ & $a_{5}$ \\
\hline $\mathrm{H}$ & $-4.91887806 \times 10^{+2}$ & $3.09155097 \times 10^{+2}$ & $-7.70038741 \times 10^{+1}$ & 9.57241011 & $-5.93380866 \times 10^{-1}$ & $1.47420199 \times 10^{-2}$ \\
\hline IH & $-2.70001339 \times 10^{+2}$ & $1.77303226 \times 10^{+2}$ & $-4.62157841 \times 10^{+1}$ & 1228 & $-3.95886800 \times 10^{-1}$ & $34 \times 10^{-2}$ \\
\hline $\mathrm{OH}$ & $-4.56875469 \times 10^{+2}$ & $2.87960316 \times 10^{+2}$ & $-7.20525364 \times 10^{+1}$ & 9.01193168 & $-5.62538083 \times 10^{-1}$ & $1.40661068 \times 10^{-2}$ \\
\hline $\mathrm{C}_{2}$ & $-1.37611619 \times 10^{+2}$ & $7.52473987 \times 10^{+1}$ & $-1.60701760 \times 10^{+1}$ & 1.72410801 & $-9.08948951 \times 10^{-2}$ & $1.90678635 \times 10^{-3}$ \\
\hline $\mathrm{CN}$ & $6.81165938 \times 10^{+2}$ & $-4.46981105 \times 10^{+2}$ & $1.17584738 \times 10^{+2}$ & $-1.53931948 \times 10^{+1}$ & 1.00366669 & $-2.60084236 \times 10^{-2}$ \\
\hline $\mathrm{MgH}$ & $6.53454485 \times 10^{+2}$ & $-4.13828321 \times 10^{+2}$ & $1.04888640 \times 10^{+2}$ & $317730 \times 10^{+1}$ & $8.31982710 \times 10^{-1}$ & $10^{-2}$ \\
\hline $\mathrm{SiH}$ & 9. & $\times 10^{+1}$ & 706604 & 0104 & $1.83429417 \times 10^{-2}$ & $10^{-4}$ \\
\hline $\mathrm{CaH}$ & $1.57677958 \times 10^{+3}$ & $-9.82846806 \times 10^{+2}$ & $2.44267425 \times 10^{+2}$ & $-3.01777064 \times 10^{+1}$ & 1.85392515 & $-4.52292569 \times 10^{-2}$ \\
\hline $\mathrm{FeH}$ & 0.1552109 & 0.3983233 & 0.6073527 & -0.198406 & $2.47056 \times 10^{-2}$ & $-9.90570 \times 10^{-4}$ \\
\hline $\mathrm{TiO}$ & $276 \times 10^{+2}$ & $492 \times 10^{+2}$ & $514 \times 10^{+1}$ & $-1.10869716 \times 10^{+1}$ & $6.75722876 \times 10^{-1}$ & $-1.63144071 \times 10^{-2}$ \\
\hline VO & $6.62090157 \times 10^{+2}$ & $-4.03350494 \times 10^{+2}$ & $9.82836218 \times 10^{+1}$ & $-1.18526504 \times 10^{+1}$ & $7.08429905 \times 10^{-1}$ & \\
\hline $\mathrm{ZrO}$ & $4.27195765 \times 10^{+2}$ & $-2.51905561 \times 10^{+2}$ & $5.85682500 \times 10^{+1}$ & -6.63032743 & $3.67462428 \times 10^{-1}$ & $-7.92597014 \times 10^{-3}$ \\
\hline
\end{tabular}

Notes. Partition functions are obtained as a function of temperature $T$ from the polynomial equation $\ln (Q)=\sum_{i=0}^{5} a_{i} \ln (T)^{i}$. 
Table A.3. Lines of Fe II, Na I, Si I, and Ca II with synflag $=Y$ or $U$, and with $g f_{-}$flag $=U$ or $N$.

\begin{tabular}{|c|c|c|c|}
\hline & Transition & $\begin{array}{l}\text { Wavelength } \\
{[\AA]}\end{array}$ & $\begin{array}{l}\text { Flags } \\
g f / \text { syn }\end{array}$ \\
\hline Fe II & $b^{4} F_{4.5}-z^{6} P_{35}^{o}$ & 4993.350 & $U / Y$ \\
\hline Fe II & $b^{4} F_{4.5}-z^{6} F_{4.5}^{o .}$ & 5132.661 & $U / U$ \\
\hline $\mathrm{Fe}$ II & $a^{6} S_{2.5}-z^{6} F_{25}^{o .9}$ & 5256.932 & $U / U$ \\
\hline Fe II & $a^{6} S_{2.5}-z^{6} F_{35}^{o}$ & 5284.103 & $U / U$ \\
\hline $\mathrm{Fe}$ II & $b^{4} G_{5.5}-w^{4} F_{4.5}^{o}$ & 5427.816 & $U / Y$ \\
\hline Fe II & $b^{2} H_{5.5}-z^{4} F_{4.5}^{o .9}$ & 5534.838 & $U / U$ \\
\hline $\mathrm{Fe}$ II & $a^{4} G_{5.5}-z^{6} F_{45}^{o .5}$ & 5991.371 & $U / Y$ \\
\hline Fe II & $a^{4} G_{4.5}-z^{6} F_{3.5}^{o .}$ & 6084.102 & $U / Y$ \\
\hline Fe II & $a^{4} G_{3.5}-z^{6} F_{25}^{o .}$ & 6113.319 & $U / U$ \\
\hline Fe II & $b^{4} D_{0.5}-z^{4} P_{0.5}^{o}$ & 6149.246 & $U / Y$ \\
\hline Fe II & $b^{4} D_{2.5}-z^{4} P_{15}^{o .}$ & 6247.557 & $U / U$ \\
\hline Fe II & $z^{4} F_{35}^{o}-c^{4} D_{3.5}$ & 6442.958 & $U / U$ \\
\hline $\mathrm{Fe}$ II & $b^{4} D_{3.5}^{5}-z^{4} P_{2.5}^{o}$ & 6456.380 & $U / Y$ \\
\hline $\mathrm{Na} \mathrm{I}$ & $z^{2} P_{1.5}^{o}-e^{2} S_{0.5}^{2.5}$ & 4751.822 & $U / U$ \\
\hline $\mathrm{Na} \mathrm{I}$ & $z^{2} P_{1.5}^{o .5}-b^{2} D_{4.5}$ & 5688.205 & $U / Y$ \\
\hline $\mathrm{NaI}$ & $z^{2} P_{0.5}^{o}-c^{2} S_{0.5}$ & 6154.225 & $U / Y$ \\
\hline $\mathrm{Na} \mathrm{I}$ & $z^{2} P_{1.5}^{o .}-c^{2} S_{0.5}$ & 6160.747 & $U / Y$ \\
\hline $\mathrm{Si}$ I & $z^{1} P_{1}^{o}-3 s^{2} 3 p\left({ }^{2} P_{3 / 2}^{o}\right) 4 f^{2}[5 / 2]_{2}$ & 5517.533 & $N / U$ \\
\hline Si I & $z^{3} D_{1}^{o}-3 s^{2} 3 p\left({ }^{2} P_{1 / 2}^{o}\right) 6 f^{2}\left[{ }^{5} / 2\right]_{2}$ & 5747.667 & $N / U$ \\
\hline Si I & $z^{3} D_{2}^{o}-3 s^{2} 3 p\left({ }^{2} P_{1 / 2}^{o}\right) 6 f^{2}\left[{ }^{5} / 2\right]_{3}$ & 5753.623 & $N / U$ \\
\hline Si I & $z^{3} D_{1}^{o}-3 s^{2} 3 p\left({ }^{2} P_{3 / 2}^{o}\right) 5 f^{2}\left[{ }^{5} / 2\right]_{2}$ & 6125.021 & $N / U$ \\
\hline Si I & $z^{3} D_{2}^{o}-3 s^{2} 3 p\left({ }^{2} P_{3 / 2}^{o}\right) 5 f^{2}\left[{ }^{5} / 2\right]_{2}$ & 6131.573 & $N / Y$ \\
\hline Si I & $z^{3} D_{2}^{o}-3 s^{2} 3 p\left({ }^{2} P_{3 / 2}^{o}\right) 5 f^{2}\left[{ }^{5} / 2\right]_{3}$ & 6131.852 & $N / Y$ \\
\hline $\mathrm{Si}$ I & $z^{3} D_{3}^{o}-3 s^{2} 3 p\left({ }^{2} P_{3 / 2}^{o}\right) 5 f^{2}\left[{ }^{5} / 2\right]_{3}$ & 6142.483 & $N / Y$ \\
\hline $\mathrm{Si}$ I & $z^{3} D_{2}^{o}-3 s^{2} 3 p\left({ }^{2} P_{3 / 2}^{o}\right) 5 f^{2}\left[{ }^{7} / 2\right]_{3}$ & 6145.016 & $N / U$ \\
\hline Si I & $z^{3} D_{3}^{o}-3 s^{2} 3 p\left({ }^{2} P_{3 / 2}^{o}\right) 5 f^{2}\left[{ }^{7} / 2\right]_{4}$ & 6155.134 & $N / U$ \\
\hline Si I & $z^{3} D_{3}^{o}-3 s^{2} 3 p\left({ }^{2} P_{3 / 2}^{o}\right) 5 f^{2}\left[{ }^{7} / 2\right]_{3}$ & 6155.693 & $N / Y$ \\
\hline Si I & $z^{1} D_{2}^{o}-3 s^{2} 3 p\left({ }^{2} P_{1 / 2}^{o}\right) 7 f^{2}\left[{ }^{7} / 2\right]_{3}$ & 6195.433 & $N / U$ \\
\hline Si I & $a^{1} D_{3}-3 s^{2} 3 p\left({ }^{2} P_{3 / 2}^{o}\right) 10 s_{1 / 2}(3 / 2,1 / 2)_{2}^{o}$ & 6208.541 & $N / U$ \\
\hline $\mathrm{Si}$ I & $z^{3} D_{1}^{o}-3 s^{2} 3 p\left({ }^{2} P_{1 / 2}^{o}\right) 5 f^{2}[5 / 2]_{2}$ & 6237.319 & $N / Y$ \\
\hline Si I & $z^{3} D_{2}^{o}-3 s^{2} 3 p\left({ }^{2} P_{1 / 2}^{o}\right) 5 f^{2}\left[{ }^{7} / 2\right]_{3}$ & 6243.815 & $N / U$ \\
\hline Si I & $z^{3} D_{2}^{o}-3 s^{2} 3 p\left({ }^{2} P_{1 / 2}^{o}\right) 5 f^{2}[5 / 2]_{3}$ & 6244.466 & $N / Y$ \\
\hline $\mathrm{Si}$ I & $z^{1} D_{2}^{o}-3 s^{2} 3 p\left({ }^{2} P_{3 / 2}^{o}\right) 6 f^{2}\left[{ }^{7} / 2\right]_{3}$ & 6414.980 & $N / U$ \\
\hline $\mathrm{Si}$ I & $a^{1} P_{1}-w^{1} D_{2}^{o}$ & 6721.848 & $U / U$ \\
\hline $\mathrm{Si}$ I & $z^{1} D_{2}^{o}-3 s^{2} 3 p\left({ }^{2} P_{3 / 2}^{o}\right) 4 f^{2}\left[{ }^{7} / 2\right]_{3}$ & 8556.776 & $N / Y$ \\
\hline Si I & $z^{3} F_{3}^{o}-3 s^{2} 3 p\left({ }^{2} P_{3 / 2}^{o}\right) 5 f^{2}[9 / 2]_{4}$ & 8556.805 & $N / Y$ \\
\hline Si I & $z^{3} F_{4}^{o}-3 s^{2} 3 p\left({ }^{2} P_{3 / 2}^{o}\right) 5 f^{2}\left[{ }^{9} / 2\right]_{5}$ & 8648.465 & $N / U$ \\
\hline Si I & $z^{3} F_{2}^{o}-3 s^{2} 3 p\left({ }^{2} P_{1 / 2}^{o}\right) 5 f^{2}\left[{ }^{7} / 2\right]_{3}$ & 8728.010 & $N / Y$ \\
\hline Si I & $z^{1} D_{2}^{o}-3 s^{2} 3 p\left({ }^{2} P_{1 / 2}^{o}\right) 4 f^{2}\left[{ }^{7} / 2\right]_{3}$ & 8742.446 & $N / Y$ \\
\hline $\mathrm{Si}$ I & $a^{1} D_{3}-3 s^{2} 3 p\left({ }^{2} P_{3 / 2}^{o}\right) 6 s_{1 / 2}\left({ }^{3} / 2,1 / 2\right)_{2}^{o}$ & 8892.720 & $U / Y$ \\
\hline $\mathrm{Si}$ I & $b^{1} D_{2}-x^{1} F_{3}^{o}$ & 8899.231 & $U / U$ \\
\hline $\mathrm{Si}$ I & $a^{1} D_{2}-3 s^{2} 3 p\left({ }^{2} P_{1 / 2}^{o}\right) 6 s_{1 / 2}^{3}\left({ }^{1} / 2,{ }^{1} / 2\right)_{1}^{o}$ & 8949.091 & $U / Y$ \\
\hline Ca II & $y^{2} P_{0.5}^{o}-d^{2} D_{1.5}$ & 5001.479 & $U / U$ \\
\hline $\mathrm{Ca}$ II & $z^{2} F_{65}^{o .3}-b^{2} G_{8.5}$ & 6456.875 & $U / U$ \\
\hline Ca II & $b^{2} D_{1.5}^{0.3}-z^{2} F_{2.5}^{o}$ & 8912.068 & $U / U$ \\
\hline Ca II & $b^{2} D_{2.5}-z^{2} F_{65}^{o .}$ & 8927.356 & $U / U$ \\
\hline
\end{tabular}


Table A.4. Atomic data for $\mathrm{Mg}$ I lines with synflag $=Y$ or $U$.

\begin{tabular}{|c|c|c|c|c|c|c|}
\hline Transition & $\begin{array}{l}\text { Wavelength } \\
{[\AA]}\end{array}$ & $\begin{array}{l}\log (g f) \\
\text { GES v6 }\end{array}$ & Ref. & $\begin{array}{l}\text { Flags } \\
g f / s y n\end{array}$ & $\begin{array}{l}\log (g f)^{(a)} \\
\text { Exp. }\end{array}$ & $\begin{array}{l}\log (g f)^{(a)} \\
\text { Calc. }\end{array}$ \\
\hline$z^{3} P_{0}^{o}-a^{3} S_{1}$ & 5167.322 & -0.931 & (1) & $Y / U$ & $-0.854 \pm 0.05$ & -0.865 \\
\hline$z^{3} P_{1}^{o}-a^{3} S_{1}$ & 5172.684 & -0.450 & (1) & $Y / Y$ & $-0.363 \pm 0.04$ & -0.387 \\
\hline$z^{3} P_{2}^{o}-a^{3} S_{1}$ & 5183.604 & -0.239 & (1) & $Y / Y$ & $-0.168 \pm 0.04$ & -0.166 \\
\hline$z^{1} P_{1}^{o}-b^{1} D_{2}$ & 5528.405 & -0.498 & (2) & $U / Y$ & $-0.547 \pm 0.02$ & -0.513 \\
\hline$z^{1} P_{1}^{o}-c^{1} S_{0}$ & 5711.088 & -1.724 & (2) & $U / Y$ & $-1.842 \pm 0.05$ & -1.742 \\
\hline$a^{3} S_{1}-w^{3} P_{2}^{o}$ & 6318.717 & -2.103 & (3) & $U / U$ & & -2.020 \\
\hline$a^{3} S_{1}-w^{3} P_{1}^{o}$ & 6319.237 & -2.324 & (3) & $U / Y$ & & -2.242 \\
\hline$a^{3} S_{1}-w^{3} P_{0}^{o}$ & 6319.493 & -2.803 & (3) & $U / Y$ & & -2.719 \\
\hline$y^{3} P_{2}^{o}-e^{3} D_{1,2,3}$ & 8717.825 & -0.866 & (3) & $U / U$ & & -0.930 \\
\hline$a^{3} D_{1,2,3}-w^{1} F_{2,3,4}^{o}$ & 8736.019 & -0.356 & (3) & $U / Y$ & & \\
\hline$z^{1} P_{1}^{o}-a^{1} D_{2}$ & 8806.757 & -0.134 & (4) & $Y / Y$ & $-0.144 \pm 0.03$ & -0.128 \\
\hline$b^{1} S_{0}-x^{1} P_{1}^{o}$ & 8923.569 & -1.678 & (2) & $U / Y$ & & -1.679 \\
\hline
\end{tabular}

References. ${ }^{(a)}$ Pehlivan Rhodin et al. (2017b). (1) Aldenius et al. (2007), uncertainties: 0.04 dex; (2) Chang \& Tang (1990); (3) Butler et al. (1993); (4) Froese Fischer \& Tachiev (2012).

Table A.5. Ni I lines with synflag $=Y$ or $U$, and with $g f_{-} f a g=U$ or $N$.

\begin{tabular}{|c|c|c|}
\hline Transition & $\begin{array}{l}\text { Wavelength } \\
{[\AA]}\end{array}$ & $\begin{array}{l}\text { Flags } \\
g f / \text { syn }\end{array}$ \\
\hline$z^{3} D_{3}^{o}-f^{3} F_{4}$ & 4806.987 & $N / U$ \\
\hline$z^{3} P_{2}^{o}-3 d^{9}\left({ }^{2} D_{5 / 2}\right) 4 d^{2}\left[{ }^{5} / 2\right]_{3}$ & 4829.023 & $N / Y$ \\
\hline$z^{3} P_{2}^{o}-3 d^{9}\left({ }^{2} D_{5 / 2}\right) 4 d^{2}\left[{ }^{1} / 2\right]_{1}$ & 4904.412 & $N / U$ \\
\hline$z^{5} F_{1}^{o}-e^{5} F_{1}$ & 4912.018 & $N / U$ \\
\hline$z^{3} P_{0}^{o}-3 d^{9}\left({ }^{2} D_{3 / 2}\right) 4 d^{2}\left[{ }^{1} / 2\right]_{1}$ & 4913.973 & $N / Y$ \\
\hline$z^{3} G_{4}^{o}-f^{3} F_{3}$ & 4918.364 & $N / Y$ \\
\hline$z^{3} G_{3}^{o}-f^{3} F_{2}$ & 4935.831 & $N / Y$ \\
\hline$z^{3} F_{2}^{o}-3 d^{9}\left({ }^{2} D_{3 / 2}\right) 4 d^{2}\left[{ }^{5} / 2\right]_{3}$ & 4946.032 & $N / Y$ \\
\hline$y^{3} F_{2}^{o}-d^{1} F_{3}$ & 4976.697 & $U / U$ \\
\hline$z^{3} F_{3}^{o}-3 d^{9}\left({ }^{2} D_{5 / 2}\right) 4 d^{2}\left[{ }^{7} / 2\right]_{4}$ & 4995.650 & $N / U$ \\
\hline$z^{3} F_{3}^{o}-3 d^{9}\left({ }^{2} D_{5 / 2}\right) 4 d^{2}\left[{ }^{5} / 2\right]_{3}$ & 5010.938 & $N / Y$ \\
\hline$z^{1} D_{2}^{o}-f^{3} F_{3}$ & 5032.727 & $N / U$ \\
\hline$z^{1} F_{3}^{o}-3 d^{9}\left({ }^{2} D_{3 / 2}\right) 4 d^{2}\left[{ }^{7} / 2\right]_{4}$ & 5081.110 & $N / U$ \\
\hline$z^{3} D_{3}^{o}-3 d^{9}\left({ }^{2} D_{5 / 2}\right) 4 d^{2}\left[{ }^{7} / 2\right]_{4}$ & 5084.096 & $N / Y$ \\
\hline$z^{3} D_{1}^{o}-3 d^{9}\left({ }^{2} D_{3 / 2}\right) 4 d^{2}\left[{ }^{1} / 2\right]_{1}$ & 5094.411 & $N / U$ \\
\hline$z^{1} D_{2}^{o}-3 d^{9}\left({ }^{2} D_{3 / 2}\right) 4 d^{2}\left[{ }^{5} / 2\right]_{2}$ & 5155.126 & $N / U$ \\
\hline$z^{1} D_{2}^{o}-3 d^{9}\left({ }^{2} D_{3 / 2}\right) 4 d^{2}\left[{ }^{5} / 2\right]_{3}$ & 5155.764 & $N / U$ \\
\hline$z^{1} D_{2}^{o}-3 d^{9}\left({ }^{2} D_{3 / 2}\right) 4 d^{2}\left[{ }^{3} / 2\right]_{2}$ & 5176.560 & $N / U$ \\
\hline$z^{3} F_{2}^{o}-3 d^{9}\left({ }^{2} D_{5 / 2}\right) 4 d^{2}\left[{ }^{7} / 2\right]_{3}$ & 5347.708 & $N / U$ \\
\hline$y^{3} D_{3}^{o}-f^{3} F_{2}$ & 5392.331 & $N / U$ \\
\hline$z^{3} D_{2}^{o}-f^{3} F_{2}$ & 5424.536 & $N / Y$ \\
\hline$z^{1} F_{3}^{o}-3 d^{9}\left({ }^{2} D_{5 / 2}\right) 4 d^{2}\left[{ }^{7} / 2\right]_{4}$ & 5462.493 & $N / U$ \\
\hline$z^{1} F_{3}^{o}-3 d^{9}\left({ }^{2} D_{5 / 2}\right) 4 d^{2}\left[{ }^{7} / 2\right]_{3}$ & 5468.104 & $N / U$ \\
\hline$z^{3} D_{1}^{o}-3 d^{9}\left({ }^{2} D_{5 / 2}\right) 4 d^{2}\left[{ }^{3} / 2\right]_{1}$ & 5475.429 & $N / U$ \\
\hline$z^{1} D_{2}^{o}-3 d^{9}\left({ }^{2} D_{5 / 2}\right) 4 d^{2}[5 / 2]_{2}$ & 5589.358 & $N / Y$ \\
\hline$z^{1} D_{2}^{o}-3 d^{9}\left({ }^{2} D_{5 / 2}\right) 4 d^{2}\left[{ }^{7} / 2\right]_{3}$ & 5593.735 & $N / Y$ \\
\hline$z^{1} P_{1}^{o}-3 d^{9}\left({ }^{2} D_{3 / 2}\right) 4 d^{2}\left[{ }^{3} / 2\right]_{2}$ & 5625.317 & $N / Y$ \\
\hline
\end{tabular}

Table A.5. continued.

\begin{tabular}{|c|c|c|}
\hline Transition & $\begin{array}{l}\text { Wavelength } \\
{[\AA]}\end{array}$ & $\begin{array}{l}\text { Flags } \\
g f / \text { syn }\end{array}$ \\
\hline$z^{1} P_{1}^{o}-e^{5} F_{1}$ & 5628.342 & $N / U$ \\
\hline$z^{1} D_{2}^{o}-3 d^{9}\left({ }^{2} D_{5 / 2}\right) 4 d^{2}\left[{ }^{3} / 2\right]_{1}$ & 5638.747 & $N / U$ \\
\hline$y^{3} F_{3}^{o}-3 d^{9}\left({ }^{2} D_{3 / 2}\right) 4 d^{2}[5 / 2]_{3}$ & 5641.881 & $N / U$ \\
\hline$z^{1} G_{4}^{o}-f^{3} F_{3}$ & 5643.078 & $N / U$ \\
\hline$y^{3} F_{3}^{o}-3 d^{9}\left({ }^{4} D_{3 / 2}\right) 4 d^{2}\left[{ }^{7} / 2\right]_{4}$ & 5682.199 & $N / Y$ \\
\hline$z^{1} P_{1}^{o}-3 d^{9}\left({ }^{2} D_{3 / 2}\right) 4 d^{2}\left[{ }^{1} / 2\right]_{1}$ & 5694.983 & $N / U$ \\
\hline$y^{3} F_{3}^{o}-f^{3} F_{4}$ & 5760.830 & $N / U$ \\
\hline$z^{3} D_{2}^{o}-3 d^{9}\left({ }^{2} D_{3 / 2}\right) 4 d^{2}\left[{ }^{5} / 2\right]_{3}$ & 5805.217 & $N / Y$ \\
\hline$y^{3} F_{2}^{o}-3 d^{9}\left({ }^{2} D_{3 / 2}\right) 4 d^{2}\left[{ }^{5} / 2\right]_{2}$ & 5996.730 & $N / U$ \\
\hline$y^{3} F_{2}^{o}-3 d^{9}\left({ }^{2} D_{3 / 2}\right) 4 d^{2}\left[{ }^{3} / 2\right]_{2}$ & 6025.754 & $N / U$ \\
\hline$y^{3} D_{1}^{o}-3 d^{9}\left({ }^{2} D_{3 / 2}\right) 4 d^{2}\left[{ }^{5} / 2\right]_{2}$ & 6086.282 & $N / Y$ \\
\hline$y^{3} F_{4}^{o}-3 d^{9}\left({ }^{2} D_{5 / 2}\right) 4 d^{2}\left[{ }^{7} / 2\right]_{4}$ & 6111.070 & $N / Y$ \\
\hline$y^{3} D_{1}^{o}-3 d^{9}\left({ }^{2} D_{3 / 2}\right) 4 d^{2}\left[{ }^{3} / 2\right]_{1}$ & 6130.135 & $N / U$ \\
\hline$y^{3} F_{4}^{o}-3 d^{9}\left({ }^{2} D_{5 / 2}\right) 4 d^{2}\left[{ }^{5} / 2\right]_{3}$ & 6133.963 & $N / U$ \\
\hline$z^{1} P_{1}^{o}-3 d^{9}\left({ }^{2} D_{5 / 2}\right) 4 d^{2}\left[{ }^{3} / 2\right]_{1}$ & 6175.367 & $N / Y$ \\
\hline$y^{3} F_{3}^{o}-3 d^{9}\left({ }^{2} D_{5 / 2}\right) 4 d^{2}[5 / 2]_{3}$ & 6186.711 & $N / Y$ \\
\hline$y^{3} F_{3}^{o}-3 d^{9}\left({ }^{2} D_{5 / 2}\right) 4 d^{2}\left[{ }^{3} / 2\right]_{2}$ & 6230.089 & $U / U$ \\
\hline$y^{3} D_{3}^{o}-3 d^{9}\left({ }^{2} D_{5 / 2}\right) 4 d^{2}\left[{ }^{7} / 2\right]_{3}$ & 6322.166 & $N / U$ \\
\hline$z^{3} P_{2}^{o}-3 d^{9}\left({ }^{2} D_{3 / 2}\right) 5 s^{2}\left[{ }^{3} / 2\right]_{2}$ & 6370.346 & $N / U$ \\
\hline$z^{3} D_{2}^{o}-3 d^{9}\left({ }^{2} D_{5 / 2}\right) 4 d^{2}\left[{ }^{3} / 2\right]_{1}$ & 6424.851 & $N / U$ \\
\hline$a^{3} P_{2}-z^{1} F_{3}^{o}$ & 6482.798 & $U / Y$ \\
\hline$y^{3} F_{2}^{o}-3 d^{9}\left({ }^{2} D_{5 / 2}\right) 4 d^{2}\left[{ }^{7} / 2\right]_{3}$ & 6598.598 & $N / Y$ \\
\hline$y^{1} F_{3}^{o}-3 d^{9}\left({ }^{2} D_{3 / 2}\right) 4 d^{2}\left[{ }^{7} / 2\right]_{4}$ & 6635.122 & $N / U$ \\
\hline$z^{3} P_{1}^{o}-3 d^{9}\left({ }^{2} D_{3 / 2}\right) 5 s^{2}\left[{ }^{3} / 2\right]_{2}$ & 6772.315 & $N / Y$ \\
\hline$z^{3} P_{1}^{o}-3 d^{9}\left({ }^{2} D_{3 / 2}\right) 5 s^{2}[3 / 2]_{1}$ & 6842.037 & $N / U$ \\
\hline$a^{1} G_{4}-y^{3} D_{3}^{o}$ & 8770.678 & $N / U$ \\
\hline
\end{tabular}




\section{Appendix B: Detailed description of atomic data}

We discuss the data sources and quality aspects for the lines of each atomic species element by element. For each element included in the preselected line list the sources for transition probabilities (oscillator strengths) and the assignment of the quality flag for $g f$-values ( $g f f l a g$ ) are discussed. This is supplemented by a discussion of the blending properties and the assignment of the corresponding quality flag (synflag). Finally, if applicable, the sources for HFS and IS data are presented. Sources for the $g f$-values of elements appearing only in the background line list are summarised in Appendix D.

The following species are included in the preselected line list (see Table 1 for an overview): H I, Li I, C I, O I, Na I, Mg I, Al I, Si I, Si II, S I, Ca I, Ca II, Sc I, Sc II, Ti I, Ti II, V I, V II, Cr I, Cr II, Mn I, Fe I, Fe II, Co I, Ni I, Cu I, Zn I, Sr I, Y I, Y II, Zr I, Zr II, Nb I, Mo I, Ru I, Ba II, La II, Ce II, Pr II, Nd II, Sm II, Eu II, Gd II, Dy II.

\section{B.1. Hydrogen $(Z=1)$}

Hydrogen being the simplest of atoms, the transition probabilities can be calculated from first principles (e.g. Gray 2005, p. 236). The source for the hydrogen Balmer and Paschen lines used here is Wiese \& Fuhr $(2009, g f f l a g=Y)$. An extended list for Paschen lines, including highly excited transitions from principal quantum numbers $>20$ can be found in Kurucz $(1993)^{21}$.

Broadening of hydrogen lines due to the presence of electrons and ions via the linear Stark effect needs to be taken into account, using for example the refined calculations by Stehlé \& Hutcheon (1999), which however in practise are quite similar to the seminal work by Vidal et al. (1973). An accurate description of resonance broadening of $\mathrm{H}$ lines due to collisions with other hydrogen atoms (self-broadening) is given in Barklem et al. (2000a), which has further been improved by Allard et al. (2008) for the case of the $\mathrm{H} \alpha 656.2 \mathrm{~nm}$ line. A recent review on hydrogen Balmer lines can be found in Barklem (2016, Sect. 4.1.1), and a ready-to-use implementation is made available by P. Barklem and N. Piskunov ${ }^{22}$.

\section{B.2. Lithium $(Z=3)$}

The $g f$-values for the two Li I $670.8 \mathrm{~nm}$ resonance fine structure transitions were taken from the theoretical calculation of Yan et al. (1998). These are essentially identical to the theoretical values quoted in Yan \& Drake (1995) and Wiese \& Fuhr (2009). The predicted life-time of the $2 \mathrm{p}^{2} \mathrm{P}^{\circ}$ upper level of these transitions from these calculations agrees extremely well with the experimental measurements of Volz \& Schmoranzer (1996) and McAlexander et al. (1996). The transition probabilities are considered accurate $(g f$ flag $=Y)$.

We adopted the highly accurate isotopic splitting data of Sansonetti et al. (1995) from frequency-modulation spectroscopy, which are in excellent agreement with the Fouriertransform spectrometry measurements of Radziemski et al. (1995). The latter authors also report HFS constants from the literature. However, HFS components were not computed for the Gaia-ESO line list. The transition probabilities for the Li I $610.3 \mathrm{~nm}$ subordinate lines in the background line list come from Lindgård \& Nielson (1977).

\footnotetext{
${ }^{21}$ Available at http://kurucz.harvard.edu/linelists/gfall/ gf0100.all.

${ }^{22}$ http://ascl.net/1507.008
}

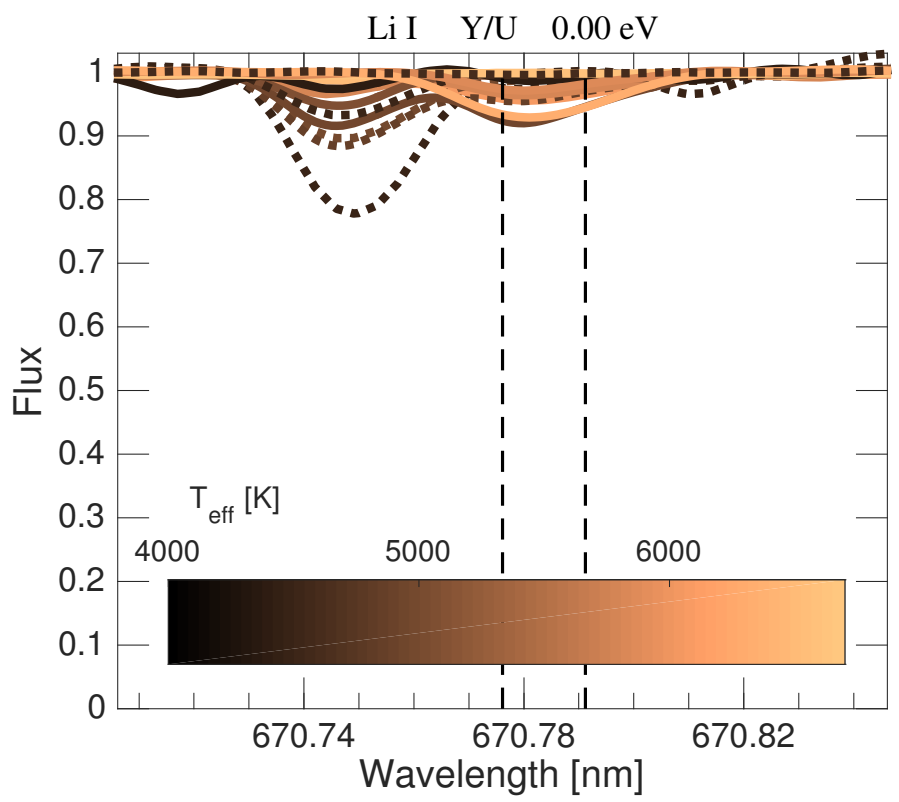

Fig. B.1. Observed spectra of selected Gaia FGK benchmark stars around the Li I $670.8 \mathrm{~nm}$ feature (see Sect. 2.3).

The $\mathrm{Li}$ I $670.8 \mathrm{~nm}$ feature is often weak and blended to varying degrees in different stars (synflag $=U$, see Fig. B.1). Lithium abundances are therefore best derived using spectral synthesis. Unfortunately, the available atomic data in the region around the Li I feature are quite poor. They cannot be used directly for abundance determination and require an astrophysical calibration. This concerns in particular two Fe I and two V I lines located within $0.4 \AA$ from the Li I feature. The two lines bluewards of Li I are by far too weak in a synthetic spectrum of the Sun and Arcturus, while the two lines redwards of Li I are by far too strong, when using the $g f$-values from Kurucz $(2007,2008)$ for Fe I and V I, respectively.

In order to provide a good basis for the Gaia-ESO analysis the following changes were made $(\log g f$-Kurucz $\rightarrow \log g f$ GES): Fe I $670.743 \mathrm{~nm},-3.917 \rightarrow-2.2$; V I $670.752 \mathrm{~nm}$, $-2.938 \rightarrow-0.8$; V I $670.809 \mathrm{~nm},-2.443 \rightarrow-2.75$; Fe I $670.828 \mathrm{~nm},-1.280 \rightarrow-2.85$. The Fe I $670.743 \mathrm{~nm}$ is one of the preselected lines (quality $N / U$ ), while the remaining lines are part of the background line list. These modifications result in a much improved synthesis for the Sun and Arcturus in this region (see Fig. B.2).

\section{B.3. Carbon $(Z=6)$}

For three permitted and one forbidden C I lines in the preselected line list, we adopted the theoretical transition probabilities of Hibbert et al. (1993). We used the Length values rather than the Velocity values as recommended by the authors, which are in good agreement with those calculated as part of the Opacity Project (Luo \& Pradhan 1989) when assuming LS coupling. Unfortunately, no recent accurate experimental measurements exist for these transitions but the theoretical values are considered reliable $(g f$ flag $=Y)$. For the [C I] $872.7 \mathrm{~nm}$ line the here adopted $\log g f$ value by Hibbert et al. (1993) is 0.03 dex larger than the recommended value by Wiese \& Fuhr (2007), which stems from the calculations of Tachiev \& Froese Fischer (2001). The transition probabilities for the other C I lines in the background line list come from Kurucz (2010b), and from NIST 

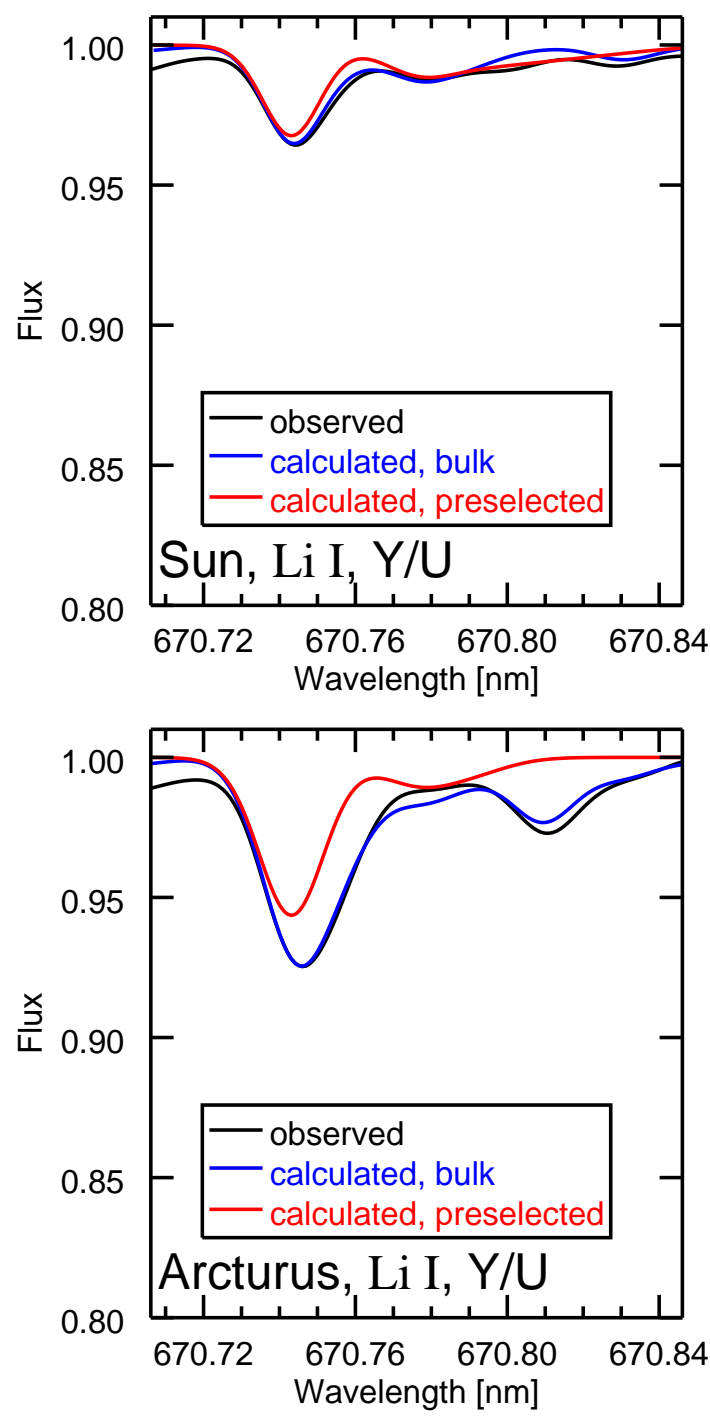

Fig. B.2. Line profiles around the Li I feature for the Sun and Arcturus. Black lines: observations, red lines: calculations including preselected spectral lines only, blue lines: calculations including blends from background line list. For Arcturus the $\mathrm{Li}$ abundance was set to $\log \left(\varepsilon_{\mathrm{Li}}\right)$ $+12=-0.8$ dex, where $\varepsilon_{\mathrm{Li}}=N_{\mathrm{Li}} / N_{\mathrm{H}}$ (e.g. Brown et al. 1989; Guiglion et al. 2016).

(Ralchenko et al. 2010), which are almost exclusively based on Hibbert et al. (1993) and Luo \& Pradhan (1989).

The four C I lines primarily used for abundance purposes are typically weak and partly blended (synflag $=U$ ) except for the $658.7 \mathrm{~nm}$ line which is considered largely clean $($ synflag $=Y$ ). Considering the low natural abundance of the ${ }^{13} \mathrm{C}$ isotope (see Table 3) neither isotopic nor HFS components were included.

\section{B.4. Oxygen $(Z=8)$}

For the [O I] $557.7 \mathrm{~nm}$ forbidden line the predictions from Baluja \& Zeippen (1988), Galavis et al. (1997) and Froese Fischer \& Tachiev (2012) agree well. They can furthermore be put on an accurate absolute scale using the life-time measurement of the $2 p^{1} \mathrm{~S}$ upper level by Corney \& Williams (1972) to yield $\log g f(557.7)=-8.241$. For the O I $615.8 \mathrm{~nm}$ line we adopted the transition probability from Hibbert et al. (1991) assuming $L S$ coupling. The adopted $g f$-values for the [O I]630.0 and $636.4 \mathrm{~nm}$ forbidden lines are the mean of the theoretical predictions of Storey \& Zeippen (2000) and Froese Fischer \& Tachiev (2012): $\log g f(630.3)=-9.715$ and $\log g f(636.3)=-10.190$. All four theoretical transition probabilities are considered reliable $(g f$ flag $=Y)$. For the other O I lines in the background line list the transition probabilities were adopted from the NIST database (Ralchenko et al. 2010), which are largely based on Hibbert et al. (1991) and the Opacity Project (Butler \& Zeippen 1991) assuming $L S$ coupling.

As discussed for example in Asplund et al. (2009), all of the four preselected O I lines are partly blended (synflag $=U$ with synflag $=N$ for the case of $557.7 \mathrm{~nm}$ ). In particular, the $\mathrm{Ni}$ I $630.0342 \mathrm{~nm}$ line in the background line list is very close to the [O I] line at $630.0304 \mathrm{~nm}$, and its $g f$-value of -2.11 was explicitly taken from Johansson et al. (2003) to replace the value of -2.674 from Kurucz (2008) contained in the VALD database.

\section{B.5. Sodium $(Z=11)$}

Accurate $\left(g f \_l a g=Y\right)$ experimental transition probabilities exist for the $\mathrm{Na}$ I $589 \mathrm{~nm}$ doublet from Volz et al. (1996). In the absence of reliable experimental data for the other preselected $\mathrm{Na}$ I lines we adopted the theoretical $g f$-values from Froese Fischer \& Tachiev (2012), which have been classified as $g f$ flag $=U$. The data for other $\mathrm{Na}$ I lines in the background line list come from the NIST database (Ralchenko et al. 2010). Besides the $\mathrm{Na} I \mathrm{D}$ lines the following three $\mathrm{Na}$ I lines are considered largely clean $($ synflag $=Y$ ): $568.8,615.4$ and $616.0 \mathrm{~nm}$.

Sodium is exclusively in the form of ${ }^{23} \mathrm{Na}$ with a nuclear spin of $3 / 2$. Sodium is thus prone to hyperfine splitting which however has not been accounted for in the Gaia-ESO line list. Suitable HFS data are available in Das \& Natarajan (2008), including the $\mathrm{Na}$ I D lines at $589 \mathrm{~nm}$.

\section{B.6. Magnesium $(Z=12)$}

The $g f$-values for optical Mg I lines are notoriously uncertain with few experimental data to rely on until recently (see discussion of new data by Pehlivan Rhodin et al. 2017b in Sect. 4.2). An exception is found for the $\mathrm{Mg}$ I $\mathrm{b}$ triplet lines, which have accurate transition probabilities provided by Aldenius et al. (2007) from measurements of life-times and branching fractions (BFs). For the other $\mathrm{Mg}$ I lines in the preselected line list we adopted theoretical values from Froese Fischer \& Tachiev (2012, line at $880.7 \mathrm{~nm}, g f \_l a g=Y$ ), from the Opacity Project (Butler et al. 1993 ) under the assumption of $L S$ coupling, or from Chang \& Tang (1990), the latter two with $g f$ flag $=U$. Several of these lines are considered largely clean (synflag $=Y$ ). For $\mathrm{Mg}$ I lines only appearing in the background line list we rely on values given by Ralchenko et al. (2010) if available and otherwise by Kurucz \& Peytremann (1975). Isotopic splitting or HFS components are not measurable in $\mathrm{Mg}$ I spectra of natural isotopic composition (Pehlivan Rhodin et al. 2017b).

\section{B.7. Aluminium $(Z=13)$}

No reliable experimental data exist for the $\mathrm{Al}$ I lines in the GaiaESO line list. We therefore resorted to using the theoretical calculations by the Opacity Project (Mendoza et al. 1995) under the assumption of $L S$ coupling with a $g f$ flag $=U$ rating for the five Al I lines in the preselected line list. The same $g f$-values were adopted by Scott et al. (2015a) in their recent analysis of the solar chemical composition. Of the available Al I lines, only $669.867 \mathrm{~nm}$ is considered largely unblended $($ synflag $=Y$ ). For other Al I lines in the background line list we make use of 
Kurucz (1975, unpublished) and Wiese et al. (1969). Aluminium consists entirely of ${ }^{27} \mathrm{Al}$, which has nuclear spin $5 / 2$. While not accounted for explicitly in the Gaia-ESO line list, good HFS data are available in for example Nakai et al. (2007) and Sur et al. (2005), see Nordlander \& Lind (2017, their Table A.2).

\section{B.8. Silicon $(Z=14)$}

When available, we adopted the experimental transition probabilities of Garz (1973) for Si I, which are however only reliable in a relative sense. Therefore we re-normalised them to an improved absolute scale in the same manner as Scott et al. (2015a) with the highly accurate, laser-induced fluorescence (LIF) life-times of the $4 \mathrm{~s}{ }^{3} \mathrm{P}_{0,1,2}$ levels measured by O'Brian \& Lawler (1991), resulting in a $g f$ flag $=Y$ rating. For a few Si I lines not available in Garz (1973), we rely on the Opacity Project calculations of Nahar (1993), which were obtained under the close-coupling approximation with the R-matrix method $(g f f l a g=U)$. These data were complemented by the extensive calculations of Kurucz $(2007, g f$ flag $=N)$ for a considerable number of lines.

For the two Si II lines at 634.711 and $637.137 \mathrm{~nm}$ in the preselected line list we adopted the same $g f$-values as in Scott et al. (2015a), which were obtained from taking the mean of Schulz-Gulde (1969), Blanco et al. (1995), and Matheron et al. (2001) and are given a $g f$ flag $=Y$ evaluation. For Si I lines only appearing in the background line list we made use of Kurucz (2007).

There are three Si I lines with a simultaneous $g f \_l a g=Y$ and synflag $=Y$ rating, at 569.043, 570.110 and $594.854 \mathrm{~nm}$, while the remainder of the Garz (1973) lines in the preselected line list are partly blended. A comparison of line abundances derived for four benchmark stars (see Sect. 2.3) for two different sets of lines (with $g f$ flag $=Y$ and $g f_{\_}$flag $=N$ ) can be seen in Fig. B.3, while mean abundances are given in Table B.1. The $g f$ flag $=N$ lines generally result in a larger scatter of line abundances than the $g f_{-} f l a g=Y$ lines, although the statistical significance is low owing to the small number of $g f_{-}$flag $=Y$ lines.

\section{B.9. Sulphur $(Z=16)$}

For the S I triplets at 674.3 and $674.8 \mathrm{~nm}$ and the lines at 675.7 and $869.4 \mathrm{~nm}$ only theoretical $g f$-values are available. We adopted the mean transition probabilities of Froese Fischer \& Tachiev (2012) and Zatsarinny \& Bartschat (2006), which are given a $g f$ flag $=U$ rating. All of these are also partly blended $($ synflag $=U)$. The $g f$-values for other S I lines in the background line list mainly come from the theoretical calculations by Biemont et al. (1993) and Kurucz (2004).

\section{B.10. Calcium $(Z=20)$}

Highly accurate experimental $g f$-values are available for most of the preselected Ca I lines $(g f f l a g=Y)$. The majority of these have been determined by Smith \& Raggett (1981). Others have been published by Smith (1988), Drozdowski et al. (1997), and Aldenius et al. (2009). We also included a few lines without experimental $g f$-values, for which we used the calculations by Froese Fischer \& Tachiev (2012) with $g f$ flag $=U$. About one third of these lines are largely blend-free in the Sun and Arcturus (synflag $=Y$, see Table 1). Two examples are illustrated in Fig. B.4. The line at $526.039 \mathrm{~nm}$ was used for most of the FGK dwarfs and giants in the abundance determination for benchmark stars by Jofré et al. (2015), and the line at $649.965 \mathrm{~nm}$ for most of the FG dwarfs and the metal-poor stars in the same study (see also Sect. 4.1).

For Ca II only few experimental $g f$-values exist. We instead rely on theoretical data from the Opacity Project (Saraph \& Storey 2012, $g f_{-}$flag $=U$ ) under the assumption of $L S$ coupling, as discussed in detail in Mashonkina et al. (2007), and on calculations by Theodosiou (1989) for the NIR triplet lines $(g f$ flag $=Y)$. For two of the NIR triplet lines the calculations by Theodosiou (1989) show excellent agreement with the experimental data by Gallagher (1967). We note that the values of Theodosiou (1989) are approximately 0.05 dex higher than those from Saraph \& Storey (2012), which were included in version 4 of the Gaia-ESO line list. Figure B.5 shows the solar observed and synthetic spectrum (see Sect. 2.3) for the NIR triplet lines, which are the most important Ca II lines in the GIRAFFE setting used by the GES. The pressure sensitivity of the lines provides an excellent gravity constraint for dwarf stars, in particular the lines at 854.2 and $866.2 \mathrm{~nm}($ synflag $=Y)$. All other preselected CaII lines are blended to some degree (synflag= $U$ or $N)$.

Data for Ca I lines in the background line list were also taken from Smith (1981) and Kurucz (2007), in addition to the references above. For Ca II lines we used additional data from Seaton et al. (1994) and Kurucz (1999). The three Ca I autoionising lines at $631.811,634.331$, and $636.175 \mathrm{~nm}$ lie within the Gaia-ESO wavelength range and are included in the background line list. To enable a realistic modelling of their Fano profiles the radiative damping parameters were assigned values derived from the Shore parameters provided by R.L. Kurucz ${ }^{23}$. The first Shore parameter is the radiative width (Shore 1967), which is given in frequency units by Kurucz as $\log _{10}\left(\Gamma_{f}\right)$, where $\Gamma_{f}$ is the full width at half maximum (FWHM) in Hz. The radiative damping parameter in the Gaia-ESO line list (and in the Kurucz lists for lines other than autoionising lines) is $\log _{10}(\Gamma)$, where $\Gamma$ is the FWHM in angular frequency units $\left(\mathrm{rad} \mathrm{s}^{-1}\right)$. Thus, to convert to the usual radiative damping parameter, one uses $\Gamma=2 \pi \Gamma_{f}$, and thus $\log _{10}(\Gamma)=\log _{10}\left(\Gamma_{f}\right)+0.80$, and this value is given in the line list.

The remaining two parameters given by Kurucz are the Shore parameters $a$ and $b$ describing the profile shape. The three lines have an asymmetry parameter $a$ of practically zero $\left(\log _{10}(a)=-30 \mathrm{~cm}^{2} \mathrm{~g}^{-1}\right)$, which implies a Lorentz absorption profile with no asymmetry (i.e. the equivalent Fano parameter $q=\infty$; see Shore 1967). Thus, an appropriate radiative damping parameter achieves the same result as the profile according to the Shore parameterisation of the Fano profile. Tests indicate that these data reproduce profiles in standard stars reasonably well. The presence of the autoionising lines affects the derivation of abundances from other lines that fall in this region, an example being Zn (see Appendix B.20).

\section{B.11. Scandium $(Z=21)$}

For both Sc I and Sc II lines the $g f$-values of Lawler \& Dakin (1989) are to be preferred ( $g f$ flag $=Y$, including all of the preselected Sc I lines). These authors determined BFs using emission FTS measurements, while the absolute scale was obtained using the time-resolved laser-induced fluorescence (TRLIF) life-times of Marsden et al. (1988). For a few preselected Sc II lines without experimental $g f$-values we used the calculations by Kurucz $(2009, g f$ flag $=N)$. The background line list also contains $g f$ values from Martin et al. (1988).

${ }^{23}$ http://kurucz.harvard.edu/atoms/2000/gf2000.all 

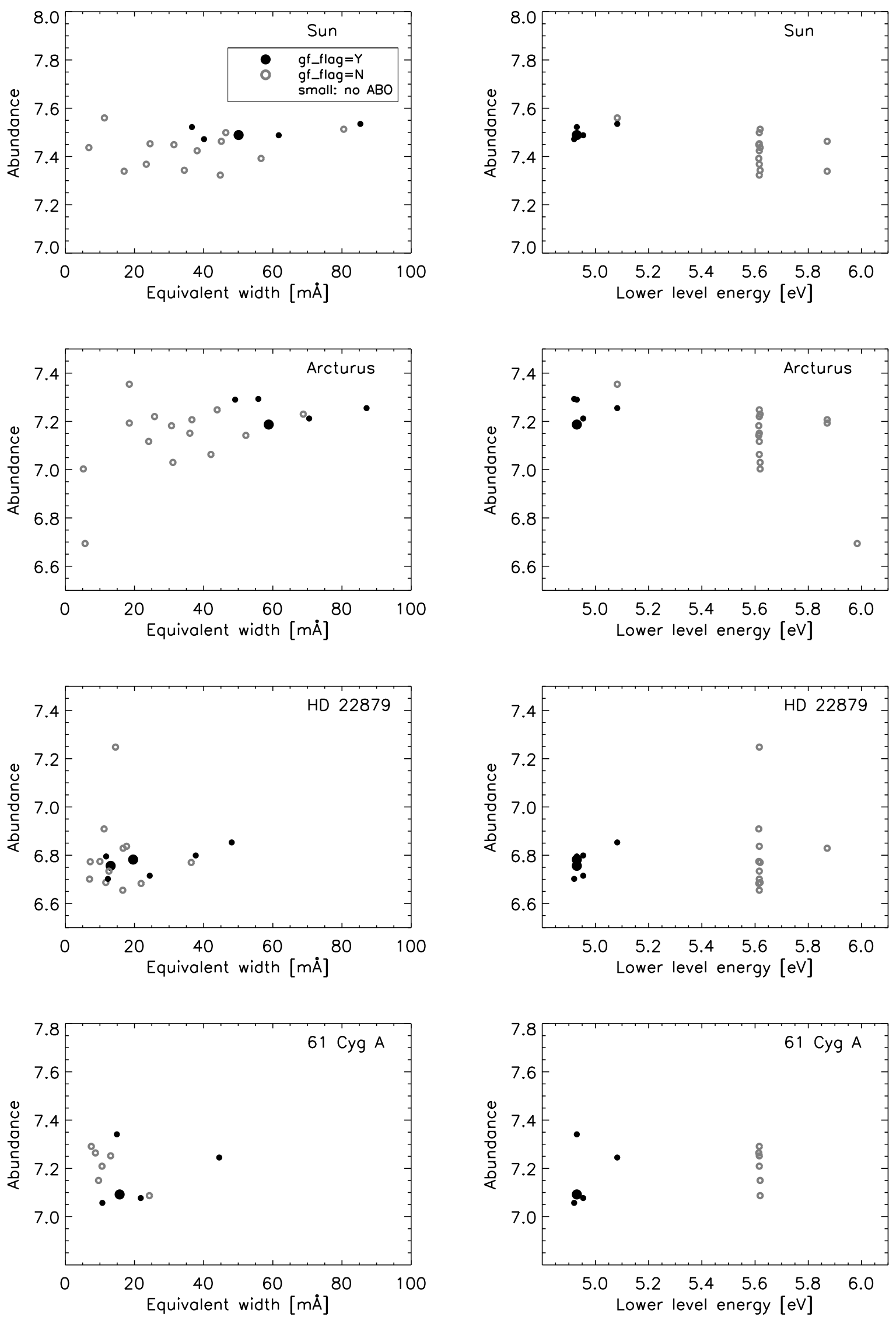

Fig. B.3. Line-by-line Si I abundances determined for four benchmark stars for two groups of lines with different quality assessment of their transition probabilities, as a function of equivalent width and lower level energy. Abundances are given as $\log \left(\varepsilon_{\mathrm{Si}}\right)+12$, where $\varepsilon_{\mathrm{Si}}=N_{\mathrm{Si}} / N_{\mathrm{H}}$. Only lines with synflag $=Y$ or $U$ and with equivalent widths $>5 \mathrm{~m} \AA$ are included. 

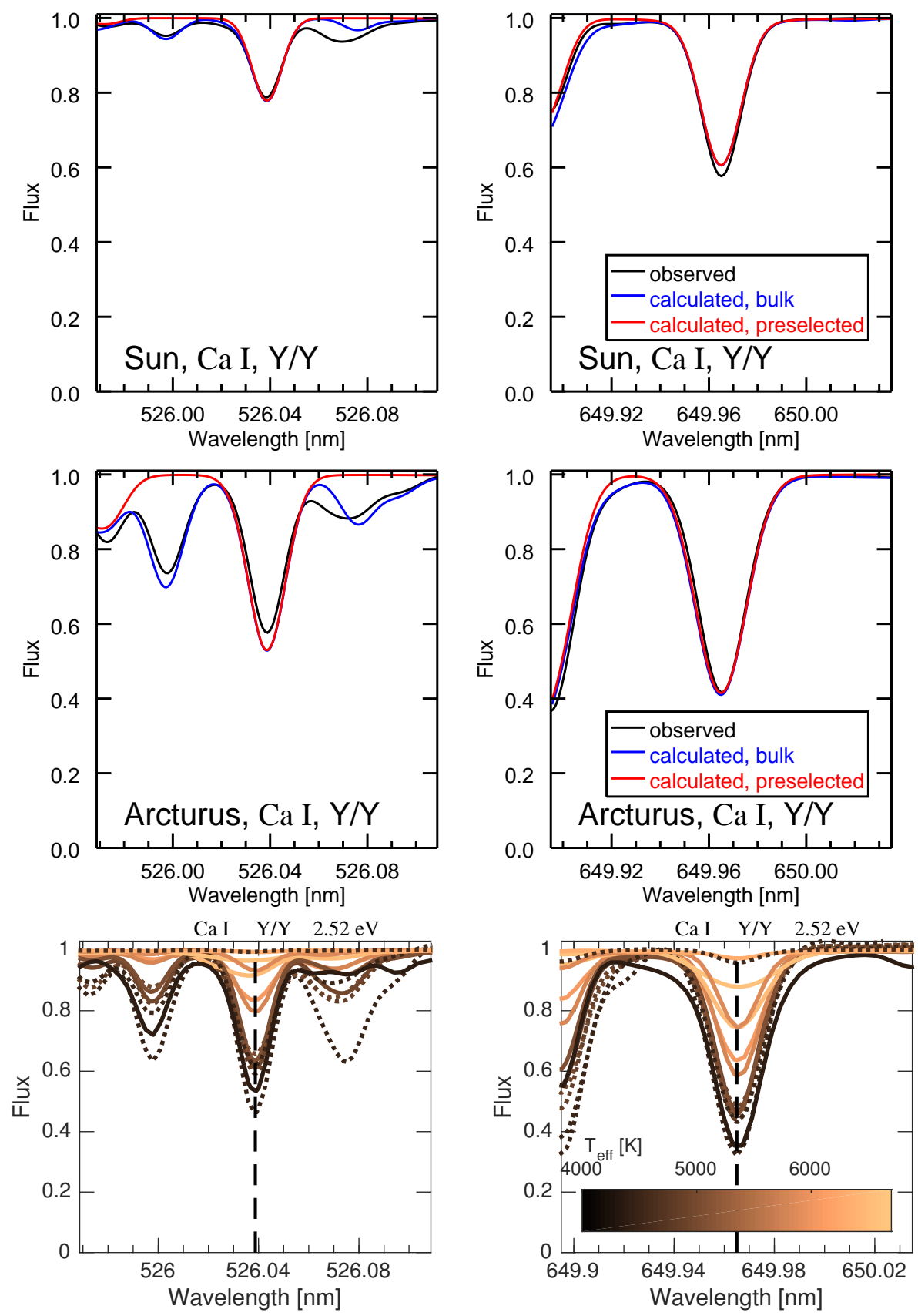

Fig. B.4. Observed and calculated line profiles around two preselected $\mathrm{Ca}$ I lines for the Sun (top row) and Arcturus (middle row). Black lines: observations, red lines: calculations including preselected spectral lines only, blue lines: calculations including blends from background line list. Bottom row: line profiles generated from observed spectra of selected Gaia FGK benchmark stars. See Sect. 2.3.

Only the two Sc I lines at 535.6 and $621.1 \mathrm{~nm}$ and the two Sc II lines with high-quality $g f$-values at 565.8 and $566.7 \mathrm{~nm}$ are blend-free (synflag $=Y$ ). Examples for Sc II line profiles are shown in Fig. B.6. The line at $565.790 \mathrm{~nm}$ was used for most of the FGK dwarfs and the metal-poor stars in the abundance determination for benchmark stars by Jofré et al. (2015), and the line at $660.460 \mathrm{~nm}$ for most of the FGK dwarfs and giants (see also Sect. 4.1).

HFS data. For Sc I a wealth of HFS data from both experimental and theoretical studies are available. Childs (1971) employed the atomic-beam magnetic-resonance technique (ABMR) resulting in highly accurate data for the $3 \mathrm{~d} 4 \mathrm{~s}^{2}{ }^{2} \mathrm{D}_{3 / 2,5 / 2}$ levels. ABMR data were also presented by Ertmer \& Hofer (1976) and Zeiske et al. (1976) for the $\left({ }^{3} \mathrm{~F}\right) 4 \mathrm{~s}{ }^{4} \mathrm{~F}_{3 / 2,5 / 2,7 / 2,9 / 2}$ levels, which we employ. For several others, including the $\left({ }^{3} \mathrm{~F}\right) 4 \mathrm{~s}{ }^{2} \mathrm{~F}_{5 / 2,7 / 2}$ levels, the only source of data are the calculations published by Başar et al. (2004). The high-excitation levels are represented by the theoretical predictions by Öztürk et al. (2007). The complete set of HFS data is given in Table C.1. For Sc II no HFS components were computed, but HFS data for most of the preselected Sc II lines can be found in Scott et al. (2015b, their Sect. 6.1.2 and Table 2).

\section{B.12. Titanium $(Z=22)$}

For Ti I we adopted transition probabilities primarily from Nitz et al. (1998) and Lawler et al. (2013), and for Ti II we preferred Wood et al. (2013). Nitz et al. (1998) measured BFs for Ti I from FTS spectra and combined those with accurate TRLIF life-times from Salih \& Lawler (1990), and Lawler et al. (2013) expanded on that work. In addition, we used accurate $g f$-values from Grevesse et al. (1989), produced by re-normalising the relative oscillator strengths from the Oxford group (Blackwell et al. 1982a, 1983, 1986) with the absolute scale fixed by using the TRLIF life-times of Rudolph \& Helbig (1982). We also used data 
Table B.1. Abundance statistics (number of lines, mean, and standard deviation of $\log \varepsilon_{\mathrm{X}}+12$, where $\varepsilon_{\mathrm{X}}=N_{\mathrm{X}} / N_{\mathrm{H}}$ ) for four species and four Gaia FGK benchmark stars.

\begin{tabular}{|c|c|c|c|c|c|c|c|c|c|c|c|c|}
\hline & \multicolumn{3}{|c|}{ Sun } & \multicolumn{3}{|c|}{ Arcturus } & \multicolumn{3}{|c|}{ HD 22879} & \multicolumn{3}{|c|}{61 Cyg A } \\
\hline & $N$ & Mean & Stdd & $N$ & Mean & Stdd & $N$ & Mean & Stdd & $N$ & Mean & Stdd \\
\hline \multicolumn{13}{|l|}{ Si I } \\
\hline$g f_{-}$flag $=Y$ & 5 & 7.50 & 0.03 & 5 & 7.25 & 0.05 & 7 & 6.77 & 0.05 & 5 & 7.16 & 0.12 \\
\hline$g f_{\text {fflag }}=N$ & 13 & 7.43 & 0.07 & 14 & 7.13 & 0.16 & 12 & 6.80 & 0.16 & 6 & 7.21 & 0.08 \\
\hline \multicolumn{13}{|l|}{ Cr I } \\
\hline$g f_{f}$ flag $=Y$ & 13 & 5.61 & 0.11 & 11 & 4.96 & 0.08 & 9 & 4.53 & 0.07 & 8 & 5.26 & 0.26 \\
\hline$g f_{-}$flag $=N$ & 9 & 5.75 & 0.20 & 10 & 5.01 & 0.19 & 3 & 4.58 & 0.06 & 11 & 5.32 & 0.21 \\
\hline \multicolumn{13}{|l|}{$\mathrm{Fe} I$} \\
\hline$g f_{f}$ flag $=Y$ & 81 & 7.52 & 0.14 & 39 & 6.88 & 0.15 & 149 & 6.42 & 0.13 & 59 & 7.07 & 0.27 \\
\hline$g f_{-}$flag $=U$ & 84 & 7.53 & 0.14 & 78 & 6.95 & 0.20 & 69 & 6.54 & 0.13 & 72 & 7.27 & 0.28 \\
\hline$g f_{\text {fflag }}=N$ & 46 & 7.69 & 0.66 & 55 & 7.04 & 0.61 & 16 & 6.52 & 0.31 & 34 & 7.43 & 0.74 \\
\hline \multicolumn{13}{|l|}{$\mathrm{Ni} \mathrm{I}$} \\
\hline$g f$ flag $=Y$ & 20 & 6.32 & 0.20 & 10 & 5.65 & 0.29 & 23 & 5.23 & 0.08 & 17 & 5.93 & 0.16 \\
\hline$g f_{\text {fflag }}=N$ & 36 & 6.16 & 0.15 & 39 & 5.57 & 0.21 & 28 & 5.17 & 0.15 & 29 & 6.01 & 0.45 \\
\hline
\end{tabular}

Notes. Only lines with synflag $=Y$ or $U$ and with equivalent widths $>5 \mathrm{~m} \AA$ and $\leq 100 \mathrm{~m} \AA$ were included. See Sect. 2.3, and Sects. B.8, B.14, B.16, and B.18.

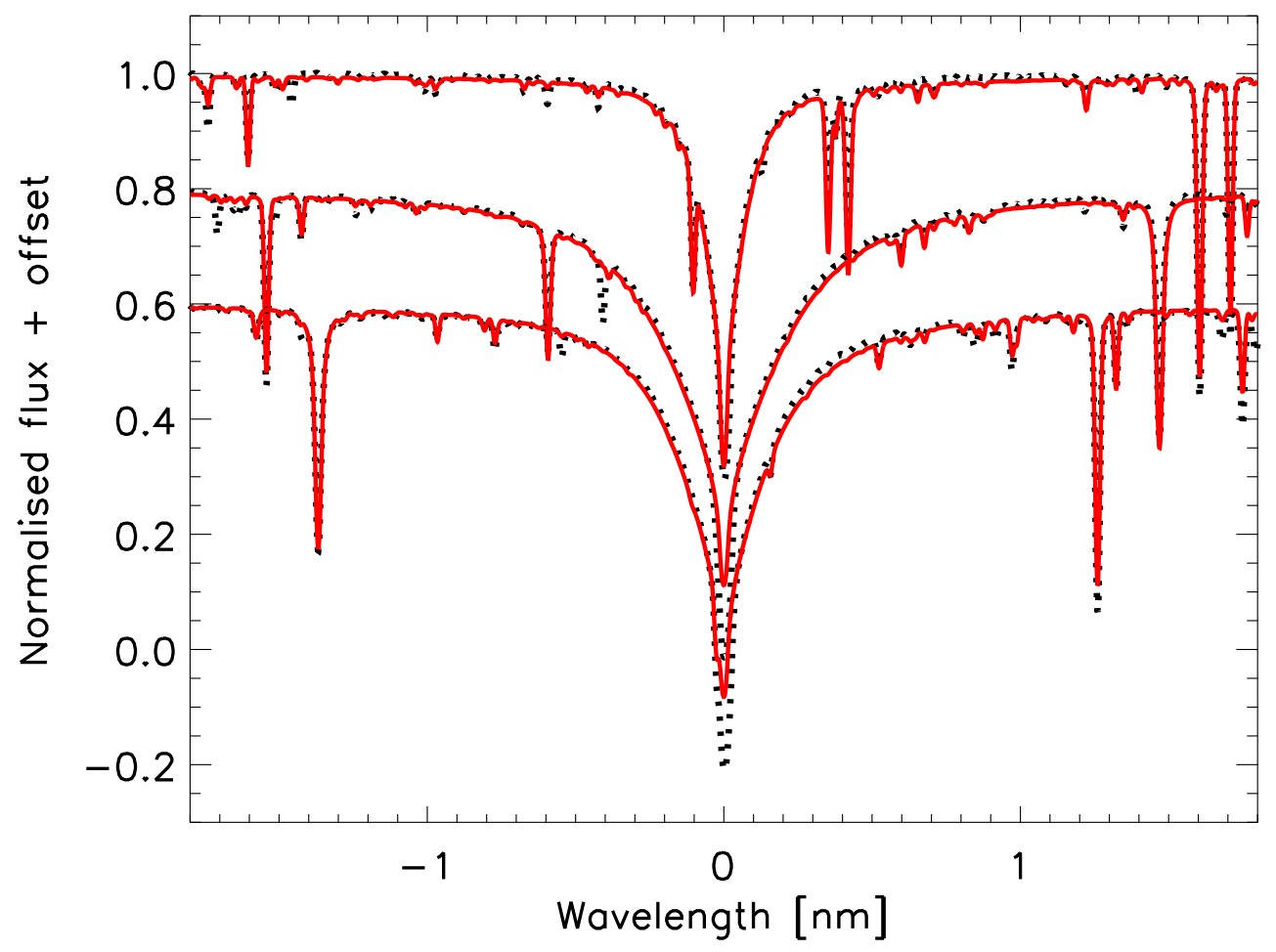

Fig. B.5. Observed (black dotted lines) and synthesised (red solid lines) solar spectra of the Ca II near-infrared (NIR) triplet lines at $849.8,854.2$, and $866.2 \mathrm{~nm}$, from top to bottom. The lines are shifted with respect to their central wavelength and offsets are added to the normalised fluxes for clarity. by Smith \& Kuehne (1978) for one line. All lines with data from these sources were assigned a $g f$ flag of $Y$. For the remaining few Ti I lines we used the semi-empirical calculations by Kurucz $(2010 b, g f$ flag $=N)$.

Most of the Ti II lines in the preselected line list are covered by the high-quality FTS and echelle work by Wood et al. (2013). Another FTS study by Pickering et al. (2001) produced $g f$-values for many lines but relied on calculated oscillator strengths of weak lines to complete the fractions for some branches. To put their BFs on an absolute scale Pickering et al. (2001) used lifetimes from Bizzarri et al. (1993) or life-times derived from the theoretical transition probabilities of Kurucz (2010b). These data were used for two of the lines. All lines from these two sources were assigned a $g f$ flag of $Y$, while the data for the remaining few Ti II lines were taken from Kurucz (2010b) with $g f_{-} f a g=N$.

The Ti I lines are the second most numerous (after Fe I) among the preselected lines (see Table 1), and $90 \%$ of them have high-quality $g f$-values. Among those, $24 \%$ are also unblended in the Sun and Arcturus (synflag $=Y$ ). In contrast, there is only one among the $18 \mathrm{Ti}$ II lines with $g f$ flag $=Y$ which also is blend-free $(541.877 \mathrm{~nm})$. Examples for line profiles are shown in Fig. B.7. The Ti I line at $597.854 \mathrm{~nm}$ was used for most of the FGK dwarfs and giants in the abundance determination for benchmark stars by Jofré et al. (2015), and the Ti II line at $487.401 \mathrm{~nm}$ for most of 

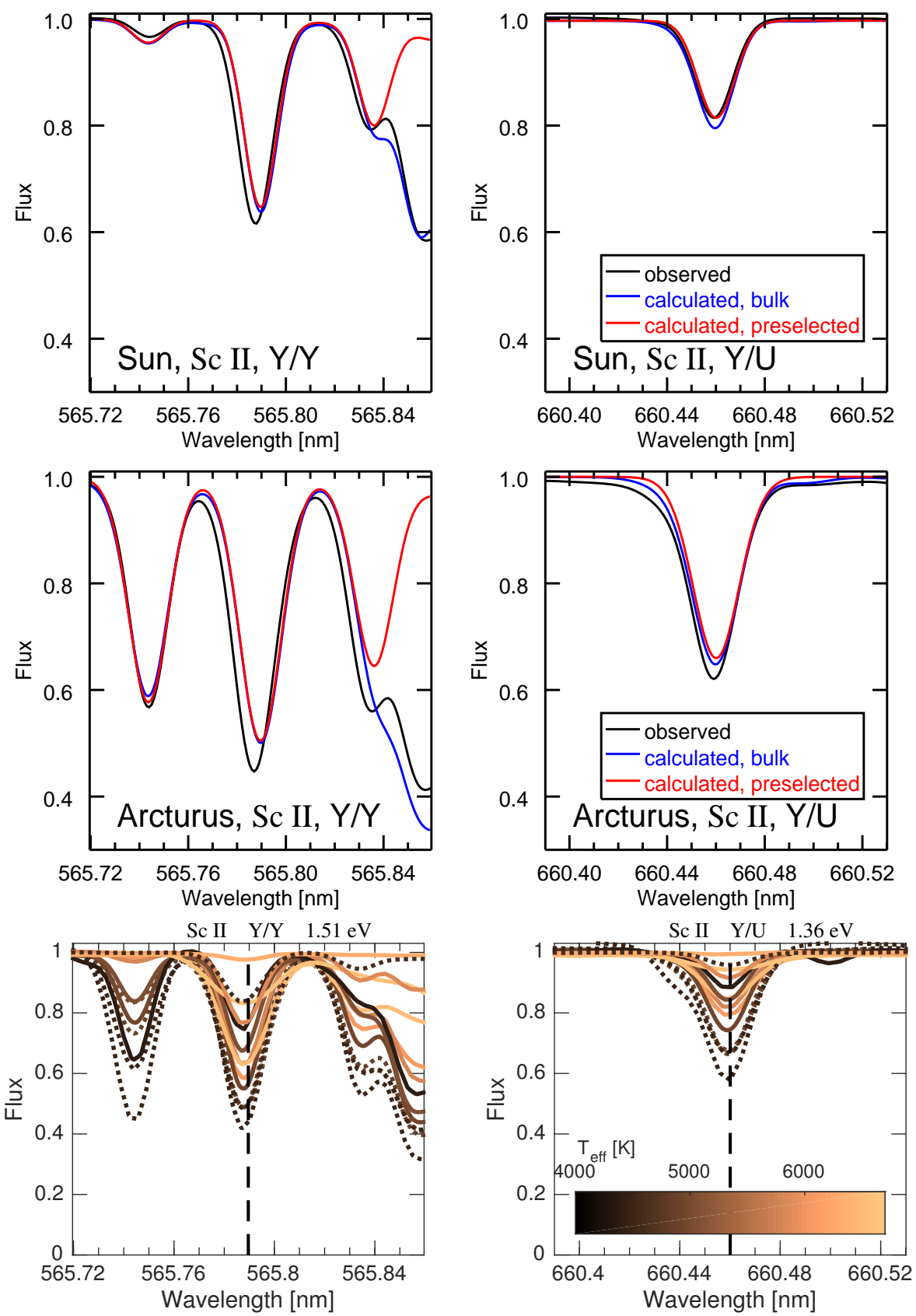

Fig. B.6. Observed and calculated line profiles around two preselected Sc II lines for the Sun (top row) and Arcturus (middle row). Black lines: observations, red lines: calculations including preselected spectral lines only, blue lines: calculations including blends from background line list. Bottom row: line profiles generated from observed spectra of selected Gaia FGK benchmark stars. See Sect. 2.3.

the FG dwarfs, the FGK giants, and the metal-poor stars in the same study (see also Sect. 4.1).

The background line list relies mainly on calculations by Kurucz (2010b), and for Ti I on data from Martin et al. (1988), Nitz et al. (1998), and Lawler et al. (2013). For a few Ti II lines in the background line list experimental data and astrophysical determinations were used (Ryabchikova et al. 1994; Pickering et al. 2001; Wood et al. 2013).

Titanium has five isotopes with non-negligible natural abundances (see Table 3), including two with non-zero spin. Isotopic splittings are presented in Scott et al. (2015b, their Sect. 6.2.2 and Table 2) for two and six of the preselected Ti I and Ti II lines, respectively, together with HFS constants. The largest wavelength shifts between two isotopes are $0.04 \AA$ for Ti I, and $<0.03 \AA$ for Ti II. None of the lines have HFS data for both levels. Therefore, neither isotopic nor HFS components were included in the Gaia-ESO line list for Ti.

\section{B.13. Vanadium $(Z=23)$}

The work of Whaling et al. (1985) provides the most accurate measurements of V I oscillator strengths to date, based on both FTS BFs and TRLIF life-times. Most of our preselected V I lines are covered by this source $\left(g f \_\right.$flag $\left.=Y\right)$, and about $40 \%$ of these lines are also unblended in the Sun and Arcturus $($ synflag $=Y$ ). Line profiles for the V I line at $611.952 \mathrm{~nm}$ are shown in Fig. B.8 (left column) as a representative example. This line was used for the largest number of FGK dwarfs and giants in the determination of the $\mathrm{V}$ abundance for benchmark stars by Jofré et al. (2015) (see also Sect. 4.1).

For 13 out of the 49 preselected V I lines and most of the lines in the background line list, the $g f$-values come from the calculations of Kurucz $(2009, g f$ flag $=N$ ), except when data are available in Martin et al. (1988). For V II all $g f$-values come from the calculations of Kurucz $(2010 \mathrm{~b}, g f$ flag $=N)$, including the three preselected lines. 

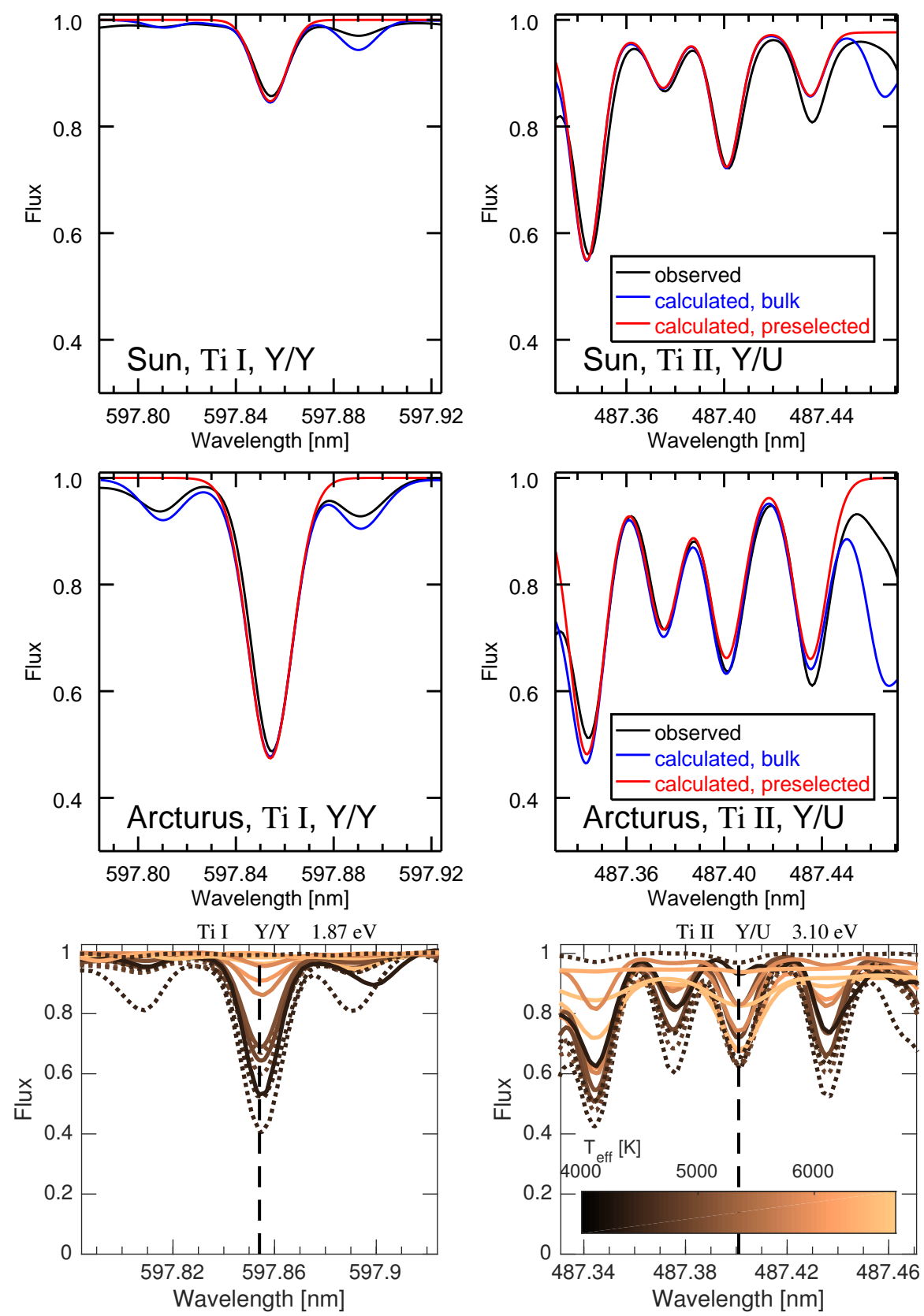

Fig. B.7. Observed and calculated line profiles around two preselected Ti I and Ti II lines for the Sun (top row) and Arcturus (middle row). Black lines: observations, red lines: calculations including preselected spectral lines only, blue lines: calculations including blends from background line list. Bottom row: line profiles generated from observed spectra of selected Gaia FGK benchmark stars. See Sect. 2.3.

HFS data. A large number of good experimental data on the HFS of V I is available, with only few levels being included in more than one study. We followed the quality assessment of Scott et al. (2015b), who sorted the data into three groups of published works with no overlap between works within each group. Data were then selected according to order of preference. The group with the most preferred data comprises the studies of Childs et al. (1979, ABMR and LFS - laser fluorescence spectroscopy - data), El-Kashef \& Ludwig (1992, ABMR), and Unkel et al. (1989, ABMR). The second-best group consists of measurements by Palmeri et al. (1995, FTS), Lefèbvre et al. (2002, FTS), and Cochrane et al. (1998, crossed beam). Data for levels not in the previous two groups were taken from Unkel et al. (1989, LFS). The complete set of HFS data is given in Table C.2.

\section{B.14. Chromium $(Z=24)$}

Sobeck et al. (2007) measured highly accurate Cr I oscillator strengths using FTS BFs normalised to TRLIF life-times of
Cooper et al. (1997). Together with the data from Blackwell et al. (1984) for one line these were assigned the $g f$ flag $=Y$. These data were complemented with experimental transition probabilities from Martin et al. (1988), Cocke et al. (1973), and Wolnik et al. (1968), and with semi-empirical $g f$-values from Kurucz (2010b), although with $g f$ flag $=N$.

Among the 35 lines with high-quality $g f$-values there are nine which also are blend-free in the Sun and Arcturus $($ synflag $=Y)$, and a further 15 lines with uncertain blend status $($ synflag $=U)$. Line abundances derived for four benchmark stars (see Sect. 2.3) for these lines are compared to line abundances for gf flag $=N$ lines in Fig. B.9, while mean abundances are given in Table B.1. For the Sun and Arcturus the scatter of the $g f_{-}$flag $=N$ lines is twice as large as the scatter of the $g f$ flag $=Y$ lines, while it is comparable for the cool dwarf star $61 \mathrm{Cyg} \mathrm{A}$, for which all of the lines might be blended to some degree.

For a few of the preselected CrII lines experimental oscillator strengths were taken from Pinnington et al. (1993, $g f$ flag $=Y$ ). For the remaining lines we used the semi-empirical 

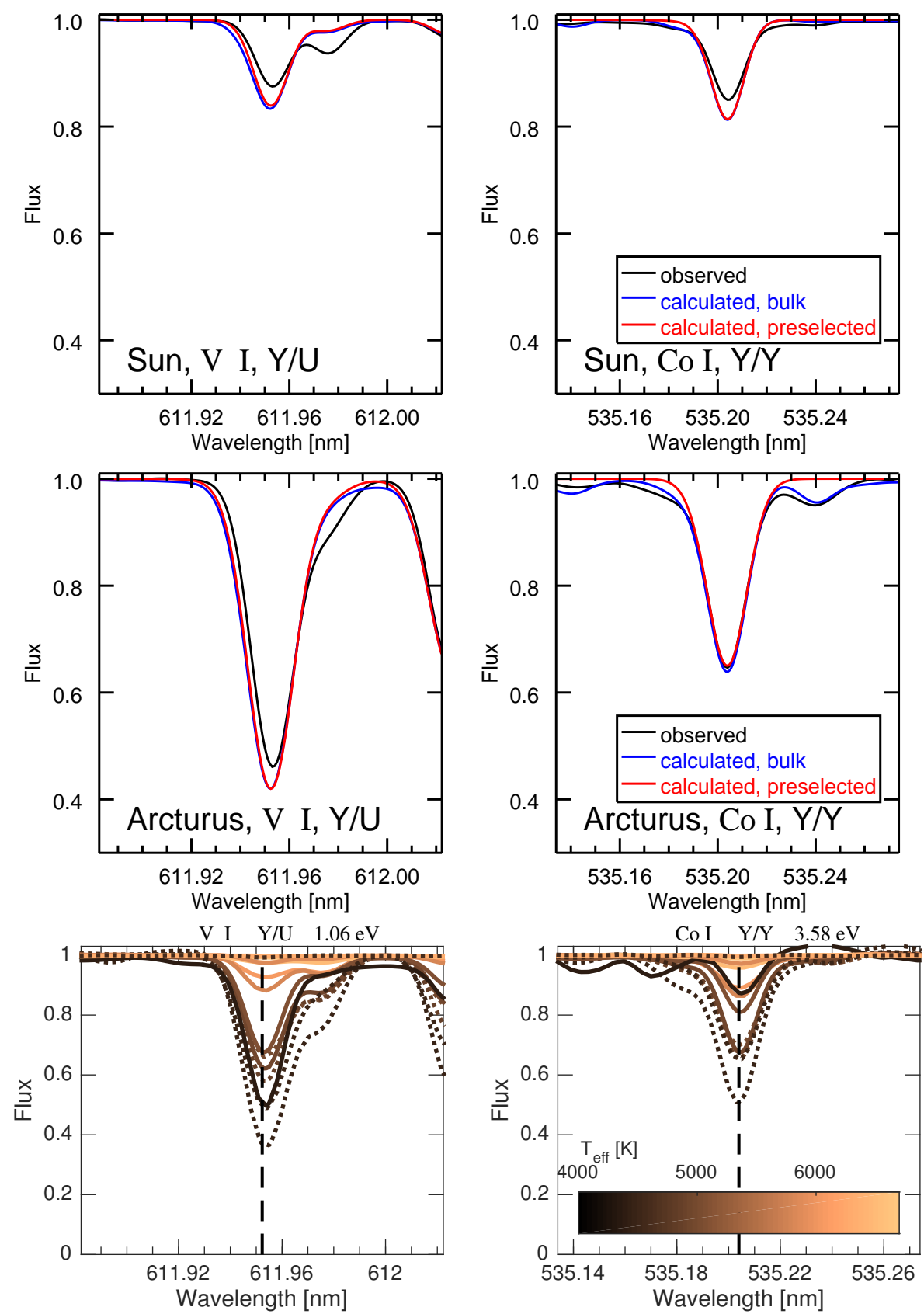

Fig. B.8. Observed and calculated line profiles around preselected lines of VI (left) and Co I (right) for the Sun (top) and Arcturus (middle). Black lines: observations, red lines: calculations including preselected spectral lines only, blue lines: calculations including blends from background line list. Bottom: line profiles generated from observed spectra of selected Gaia FGK benchmark stars. See Sect. 2.3 .

oscillator strengths from Kurucz $(2010 b, g f f l a g=N)$. More than half of the $\mathrm{Cr}$ II lines are heavily blended in the Sun and Arcturus (synflag $=N$ ), and among the remaining ones there is only one with synflag $=Y(530.585 \mathrm{~nm})$, which unfortunately does not have an experimental $g f$-value (see also Sect. 4.2). In the background line list the majority of the $\mathrm{Cr}$ II $g f$-values come from Raassen \& Uylings (1998), supplemented by data from Sigut \& Landstreet (1990), Martin et al. (1988), and the two sources above.

Chromium has four isotopes with non-negligible natural abundances (see Table 3), including one with non-zero spin. Isotopic splitting and HFS data for $\mathrm{Cr}$ are scarce and are given for one Cr I line in Scott et al. (2015b, their Sect. 6.4.2 and Table 2), which is not among our preselected lines. The wavelength difference between the lightest and heaviest $\mathrm{Cr}$ isotope is just $0.003 \AA$ and can be ignored in the context of the GES.

\section{B.15. Manganese $(Z=25)$}

For Mn I lines we used accurate experimental $g f$-values from Den Hartog et al. (2011) and Blackwell-Whitehead \& Bergemann (2007). For some lines data were taken from Booth et al. (1984). See Scott et al. (2015b) for a more detailed discussion of these sources. They cover about three quarters of the preselected lines, and all of them were assigned a $g f$ flag $=Y$. For the remaining preselected Mn I lines we resorted to the calculations of Kurucz $(2007, g f$ flag $=N)$. In addition, the background line list contains Mn I data from Martin et al. (1988) and Blackwell-Whitehead et al. (2005a), and Mn II lines with calculated $g f$-values from Kurucz (2009).

We note that for three MnI lines (601.349, 601.664, $602.179 \mathrm{~nm}$ ) the upper level energy values in Den Hartog et al. (2011) are incorrect in the fifth digit, leading to a shift in 

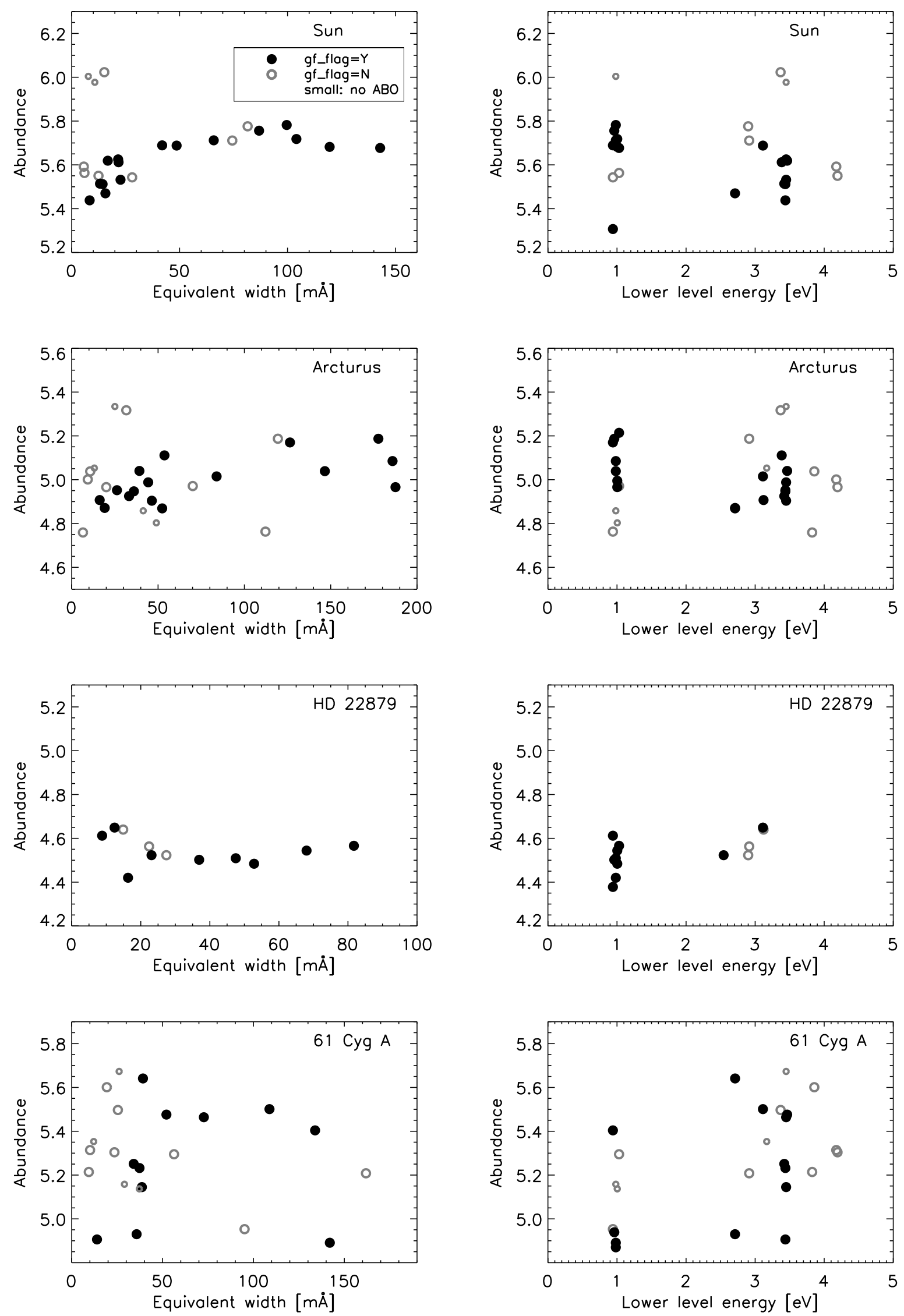

Fig. B.9. Line-by-line $\mathrm{Cr}$ I abundances determined for four benchmark stars for two groups of lines with different quality assessment of their transition probabilities, as a function of equivalent width and lower level energy. Abundances are given as $\log \left(\varepsilon_{\mathrm{Cr}}\right)+12$, where $\varepsilon_{\mathrm{Cr}}=N_{\mathrm{Cr}} / N_{\mathrm{H}}$. Only lines with synflag $=Y$ or $U$ and with equivalent widths $>5 \mathrm{~m} \AA$ are included. 

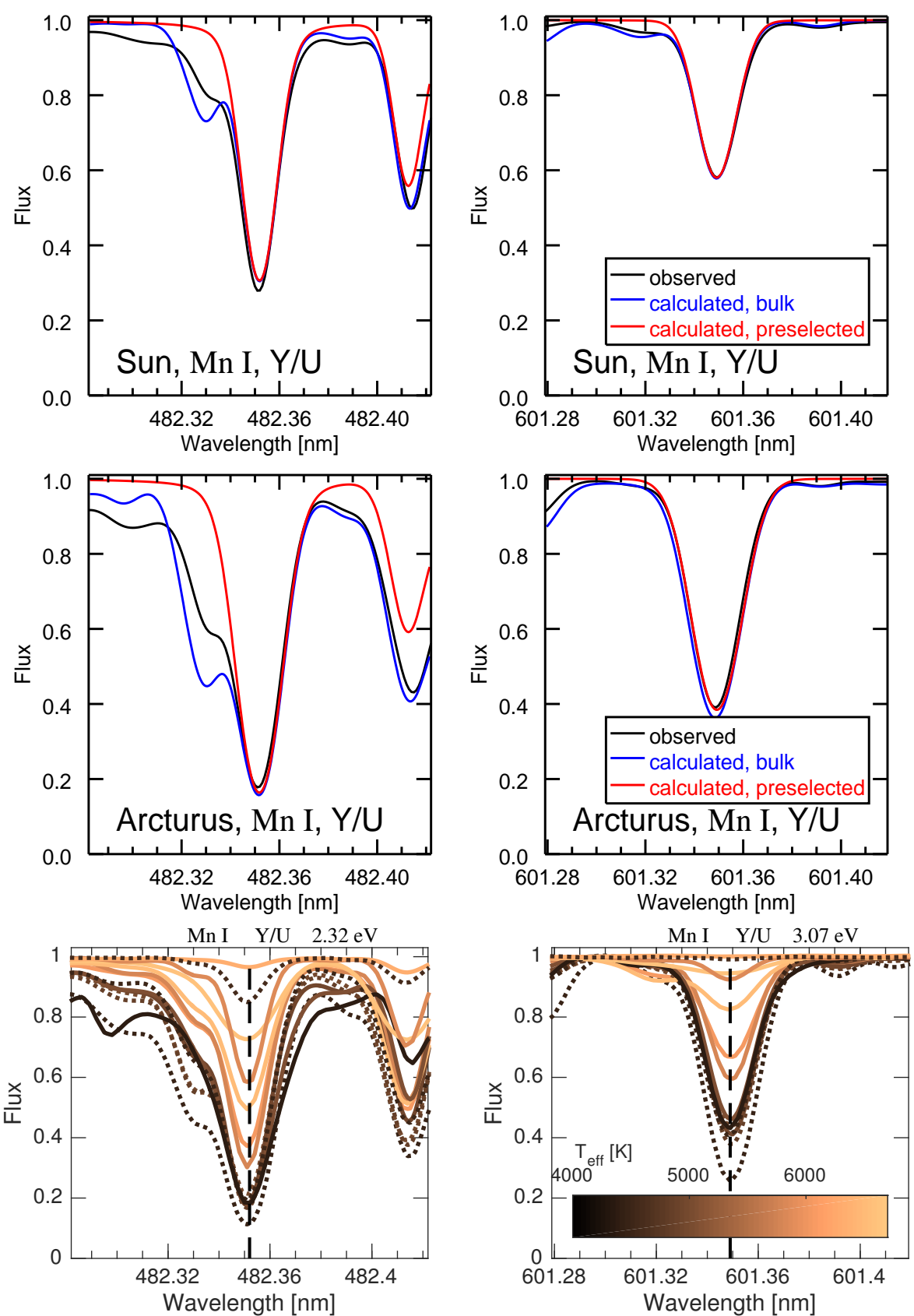

Fig. B.10. Observed and calculated line profiles around two preselected MnI lines for the Sun (top row) and Arcturus (middle row). Black lines: observations, red lines: calculations including preselected spectral lines only, blue lines: calculations including blends from background line list. Bottom row: line profiles generated from observed spectra of selected Gaia FGK benchmark stars. See Sect. 2.3.

wavelength of 0.02 to $0.03 \AA$ (see Jofré et al. 2017). The wavelengths adopted in the line list are therefore taken from Kurucz (2007).

Only three of the Mn I lines with high-quality $g f$-values have synflag $=Y(542.035,539.467,602.179 \mathrm{~nm})$, but a further 15 may be useful for abundance analysis if a careful evaluation of blending lines is done $($ synflag $=U)$. Two of these lines are shown in Fig. B.10. The line at $482.352 \mathrm{~nm}$ was used for most of the metalpoor stars in the abundance determination for benchmark stars by Jofré et al. (2015), and the line at $601.349 \mathrm{~nm}$ for most of the FGK dwarfs and giants in the same study (see also Sect. 4.1).

HFS data. For the HFS data we again followed the quality assessment of Scott et al. (2015b) and selected accurate experimental data from several groups of publications, in the following order of preference: (1) Davis et al. (1971, spinexchange), Johann et al. (1981, ABMR), Dembczyński et al.
(1979, ABMR), and Brodzinski et al. (1987, interference spectroscopy); (2) Başar et al. (2003, optogalvanic spectroscopy) for the $z^{4} D$ level, Blackwell-Whitehead et al. (2005b, FTS) for all other levels; (3) Lefèbvre et al. (2003, FTS), Luc \& Gerstenkorn (1972, FTS and Fabry-Perot spectrometry), and Handrich et al. (1969). The complete set of HFS data is given in Table C.3.

\section{B.16. Iron $(Z=26)$}

The first choice for the source of $g f$-values for Fe I lines are the publications by three laboratory spectroscopy groups from the 1980s and 1990s. The groups are those based in Oxford, UK (Blackwell et al. 1979a,b, 1982b,c, 1986), Madison, Wisconsin (O’Brian et al. 1991), and Hannover, Germany (Bard et al. 1991; Bard \& Kock 1994). The Oxford group used absorption spectroscopy to determine very precise relative $g f$-values, which were put on an absolute scale using one line with an accurate 
$( \pm 0.02$ dex $)$ transition probability. The high-quality Madison and Hannover $g f$-values are based on emission spectroscopy, which provided life-times and BFs.

For the Madison dataset the $g f$-values were calculated from the original published data (vacuum wavenumber $\bar{v}$ in $\mathrm{cm}^{-1}$, transition probability $A_{\mathrm{ul}}$ in $\mathrm{s}^{-1}$, and upper level rotational quantum number $J_{\text {upp }}$ ), using the following equation:

$g f=\frac{m_{\mathrm{e}} c}{2 \pi e^{2} \cdot 4 \pi 10^{-7} c^{2}} \cdot\left(10^{-2} / \bar{v}\right)^{2} \cdot\left(2 J_{\mathrm{upp}}+1\right) \cdot A_{\mathrm{ul}}$,

where $m_{\mathrm{e}}, c$, and $e$ are the electron mass, the speed of light, and the electron charge, respectively, all in SI units (see e.g. Gray 2005).

Recently, Ruffoni et al. (2014) and Den Hartog et al. (2014) used a similar technique to determine transition probabilities for 142 and $203 \mathrm{Fe}$ I lines, respectively, about 70 and 60 of which fall in the main Gaia-ESO wavelength regions (UVES-580 and GIRAFFE HR21 settings), and 35 and 37 of which are among the preselected lines. They focused on lines with high values of lower level energy, 3-6 eV, with few measurements reported by the above mentioned groups.

For lines in common between all of the above sources the $\log g f$-values were averaged, using weights based on the experimental uncertainties. In total, we obtained data from these sources for $45 \%$ of the $545 \mathrm{Fe}$ I lines in the preselected line list, and assigned the $g f$ flag $Y$ to them.

For lines not present in any of the above sources $g f$-values from May et al. (1974) were used if available (28\% of the preselected lines). These were assigned the $g f$ flag $U$, except for ten high-excitation lines with small uncertainties ( 0.05 dex or less), which are also unblended (synflag $Y$ or $U, g f$ flag $=Y$ ). We stress here that we elected to adopt the original values of May et al. (1974) rather than those subsequently re-normalised by Fuhr et al. (1988) based on more recent life-time measurements. This choice was made after realising that in the wavelength region covered by the GES data the original $g f$-values agree significantly better with our four preferred sources for lines in common, as illustrated in Fig. B.11.

For a few lines we used older laboratory data from Garz \& Kock (1969), Richter \& Wulff (1970), and Wolnik et al. $(1970,1971)$, choosing this time to trust the re-normalisation by Fuhr et al. (1988). We have not rigorously investigated the performance of original and re-normalised data in this case, but assigned $g f$ flag $U$ in all cases. The same ranking was made for four lines from Bridges \& Kornblith (1974) and Kock et al. (1984) with absolute values re-normalised by Fuhr \& Wiese (2006). For the remaining $25 \%$ of the preselected lines we used the semi-empirical calculations from Kurucz $(2007, g f f l a g=N)$.

Figure B.12 illustrates how well lines with different $g f$ flags perform with respect to each other in an abundance analysis. Line abundances derived for four benchmark stars (see Sect. 2.3) for 240-290 Fe I lines with synflag $Y$ or $U$ and equivalent widths $>5 \mathrm{~m} \AA$ are shown, while mean abundances for lines with equivalent widths $\leq 100 \mathrm{~m} \AA$ are given in Table B.1. A significant increase in scatter can be seen for lines with the poorest ranking, which supports our recommendations. Abundances for lines with $g f$ flag $=N$ deviate from the mean by up to 2 dex. However, also among $g f$ flag $=U$ and $Y$ lines with small quoted laboratory uncertainties there are individual exceptions with poor astrophysical performance. Examples are the $g f$ flag $=Y$ lines at $506.008 \mathrm{~nm}(0.0 \mathrm{eV}$, synflag $=U$, equivalent widths of 68,174 , $16,117 \mathrm{m \AA}$ in the Sun, Arcturus, HD 22879, and 61 Cyg A,

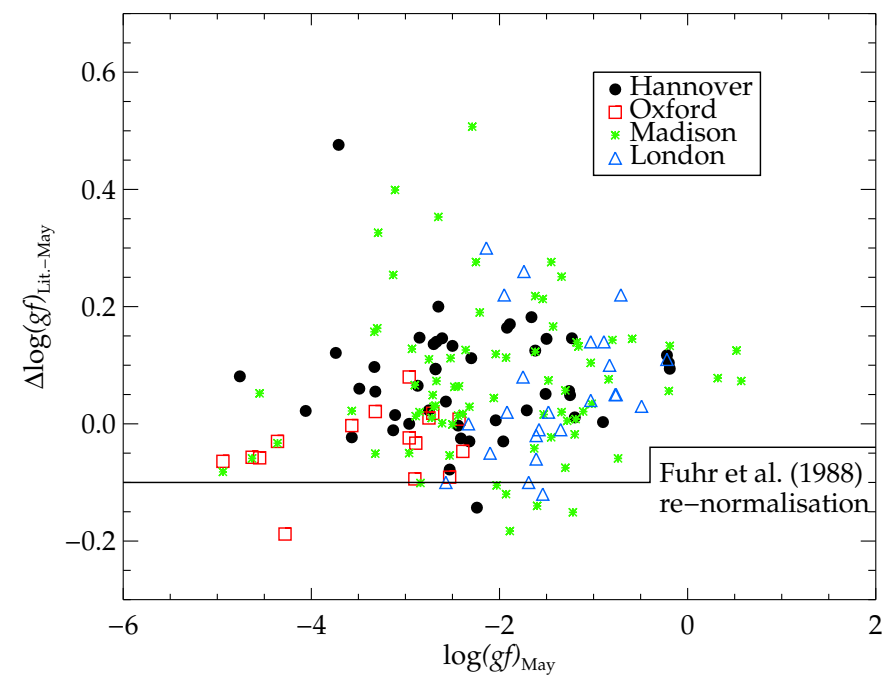

Fig. B.11. Comparison of Fe I transition probabilities published by May et al. (1974) to newer, more accurate data from the Oxford, Madison, Hannover, and London groups (see text for references). The solid line indicates the proposed normalisation of the May et al. (1974) values by Fuhr et al. (1988). Applying this normalisation would shift the zeropoint on the vertical axis to the solid line (while the data points would remain at the same positions relative to the solid line). All published spectral lines in the wavelength region 476 to $895 \mathrm{~nm}$ are shown.

respectively) and at $522.318 \mathrm{~nm}(3.635 \mathrm{eV}$, synflag $=Y$, equivalent widths of $28,56,6$, and $38 \mathrm{~m} \AA$ in the four stars), which result in $\sim 0.5$ dex higher ${ }^{24}$ and lower abundances than other $g f \_$flag $=Y$ lines with similar equivalent widths, respectively.

The most accurate Fe II $g f$-values come from the compilation of Meléndez \& Barbuy (2009). Their data are based on relative $g f$-values within each multiplet taken from theoretical works, and a calibration using experimental life-times (Hannaford et al. 1992; Schnabel et al. 1999, 2004) and BFs (e.g. Kroll \& Kock 1987; Heise \& Kock 1990). Solar calibrated values from Meléndez \& Barbuy (2009) were not considered here. This source is the preferred choice and was used for 19 preselected lines, for which the $g f$ flag was set to $Y$. For the remaining 23 preselected lines the $g f$-values were taken from the theoretical work of Raassen \& Uylings (1998), and the $g f$ flag was set to $U$.

In the background line list the majority of the $\mathrm{Fe}$ I $g f$-values are based on the semi-empirical calculations by Kurucz (2007). They are supplemented by data from May et al. (1974) and Fuhr et al. (1988), as well as the Oxford, Madison, Hannover, and London groups. We assigned a $g$ fflag $=Y$ to $148 \mathrm{Fe}$ I lines in the background line list, which have data from the latter, 'first choice' $g f$-value sources (while the synflag is not defined for any of these lines). We used theoretical data from Kurucz (2013) for about one third of the Fe II $g f$-values, in addition to the data from Raassen \& Uylings (1998).

The overall situation of transition probabilities for Fe lines was investigated by Lind et al. (2017, their Sect. 2.2.2 and Fig. 4). They compared semi-empirical calculations made available by R.L. Kurucz in 2014 with available experimental data for about $2000 \mathrm{Fe}$ I lines (out of more than 500000 possible radiative bound-bound transitions) and found differences of up to \pm 1 dex for transitions with $\log g f \gtrsim-2$, which increased to -3 dex for transitions with $\log g f \sim-4$ and high upper level energies. The compilation of the line list presented here was completed shortly

${ }^{24}$ Sun and Arcturus only. 
U. Heiter et al.: Atomic data for the Gaia-ESO Survey
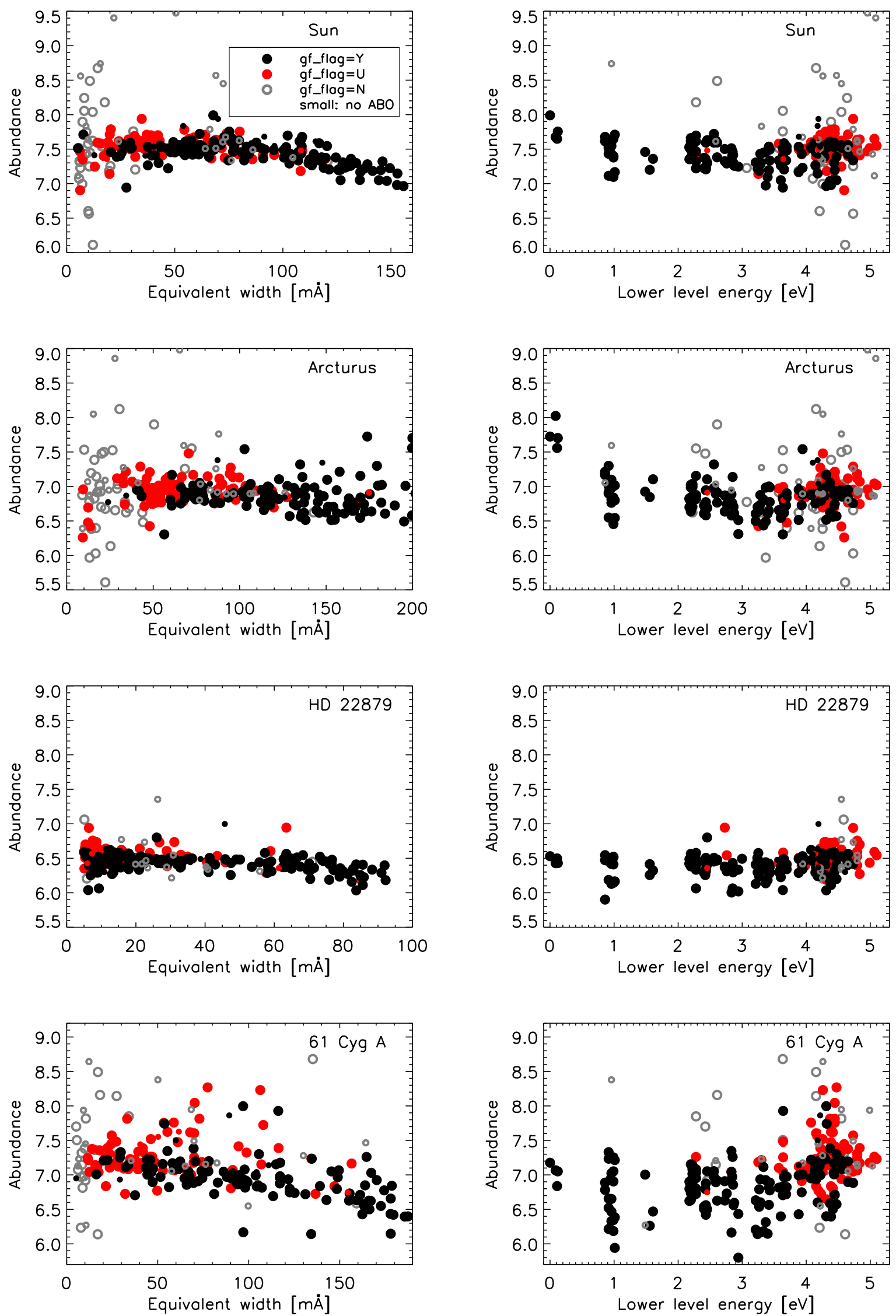

Fig. B.12. Line-by-line Fe I abundances determined for four benchmark stars for three groups of lines with different quality assessment of their transition probabilities, as a function of equivalent width and lower level energy. Abundances are given as $\log \left(\varepsilon_{\mathrm{Fe}}\right)+12$, where $\varepsilon_{\mathrm{Fe}}=N_{\mathrm{Fe}} / N_{\mathrm{H}}$. Only lines with synflag $=Y$ or $U$ and with equivalent widths $>5 \mathrm{~m} \AA$ are included. 
before the new calculations by Kurucz for Fe I became available, which is the reason why the previous calculations from 2007 were adopted. A comparison of the Kurucz data from 2007 with experimental data results in a figure which is very similar to Fig. 4 of Lind et al. (2017), with only a few more deviating points appearing at $\log g f \lesssim-5$. A direct comparison of the 2007 data with the 2014 data shows that the $g f$-values have changed by more than 0.5 dex for only about 50 out of 2000 lines.

We note that any line list is prone to being incomplete, as only about half of the lines visible in high-quality solar spectra have identified counterparts with good wavelengths according to Kurucz (2014). Many of these lines are expected to stem from iron, as indicated in the recent works by Peterson \& Kurucz (2015) and Peterson et al. (2017), who present Fe I lines newly identified from an analysis of carefully selected stellar spectra. About 1200 of these lines fall in the wavelength range considered here, and for about $20 \%$ of them the authors provide astrophysically-determined $g f$-values. While the nature of the GES speaks against the usage of astrophysical $g f$-values, as explained in Sect. 2.2, these data should be included in future line lists of other projects, potentially providing for more realistic synthetic spectra.

\section{B.17. Cobalt $(Z=27)$}

The most recent accurate Co I oscillator strengths were determined by Nitz et al. (1999) from FTS BFs put on an absolute scale using their own TRLIF life-time measurements (Nitz et al. 1995). Reliable data are also available from Cardon et al. (1982). These are the $g f$ flag $=Y$ sources used for $70 \%$ of the preselected Co I lines, supplemented by calculations of Kurucz (2008, $g f$ flag $=N$ ). The overall quality status for preselected Co I lines is similar to that of CrI with eight synflag $=Y$ lines among those with high-quality $g f$-values, and a further 14 lines with synflag $=U$ (see Table 1). Line profiles for the Co I line at 535.204 nm are shown in Fig. B.8 (right column) as a representative example. This line was used for the largest number of FGK dwarfs and giants in the determination of the Co abundance for benchmark stars by Jofré et al. (2015) (see also Sect. 4.1). In addition, the background line list contains Co I data from Martin et al. (1988) and Lawler et al. (1990), and Co II lines with calculated $g f$-values from Raassen et al. (1998) and Kurucz (2006).

HFS data. For Co I HFS we used the data measured by Pickering (1996), who used more than 1000 line profiles acquired with the high-resolution FTS at Imperial College. This yielded HFS $A$ and $B$ constants for 297 energy levels, comprising almost all known Co I energy levels (Pickering 1996). The complete set of HFS data is given in Table C.4.

\section{B.18. Nickel $(Z=28)$}

Our preferred Ni I oscillator strengths come from the recent work by Wood et al. (2014), who measured FTS and echelle spectrograph BFs and used the TRLIF life-times of Bergeson \& Lawler (1993) to provide the absolute scale. These were available for 31 preselected Ni I lines. For the Ni I line at $481.198 \mathrm{~nm}$ the highquality $g f$-value from Johansson et al. (2003) was used, which was derived from FTS BFs and a single TRLIF life-time. For the line at $542.465 \mathrm{~nm}$ the $g f$-value was taken from Doerr \& Kock (1985). Data from the above sources were assigned the gfflag $Y$. For four lines we used somewhat older experimental data from Lennard et al. $\left(1975, g f \_l a g=U\right)$. For the remaining
62 preselected lines we resorted to calculations by Kurucz (2008, gf_flag $=N$ ).

About one third of the latter are blend-free in the Sun and Arcturus (synflag $=Y$ ), and one half have uncertain blend status $($ synflag $=U)$. These lines should be given high priority for new laboratory measurements of $g f$-values (see Sect. 4.2). However, $\mathrm{Ni}$ abundances may be determined with confidence from the currently available data, as about half of the lines with highquality $g f$-values also have synflag $=Y$, and the other half has synflag $=U$ (see Table 1$)$.

Line abundances derived for four benchmark stars (see Sect. 2.3) for Ni I lines with synflag $Y$ or $U$ and equivalent widths $>5 \mathrm{~m} \AA$ are shown in Fig. B.13, while mean abundances for lines with equivalent widths $\leq 100 \mathrm{~m} \AA$ are given in Table B.1. For the Sun and Arcturus the scatter around the mean value is similar for the $g f \_$flag $=Y$ and $g f$ flag $=N$ lines, but the $N$ lines result in a systematically lower mean abundance by $\sim 0.1$ dex than the $Y$ lines. We note, however, that all the $N$ lines are high-excitation lines $\left(E_{\text {low }} \sim 4 \mathrm{eV}\right)$, while most of the $Y$ lines are low-excitation lines $\left(E_{\text {low }} \lesssim 2 \mathrm{eV}\right)$. Therefore, the abundance difference may be related to modelling deficiencies rather than atomic data issues. For HD 22879 and 61 Cyg A the mean abundances of the different sets of lines are more similar, but the $N$ lines show a twice as large scatter than the $Y$ lines.

Nickel consists of five stable isotopes, dominated by ${ }^{58} \mathrm{Ni}$ and ${ }^{60} \mathrm{Ni}$ in the Solar System (see Table 3 ). The single isotope with non-zero spin contributes only $1 \%$ to the natural $\mathrm{Ni}$ abundance, and thus HFS can be ignored. Isotopic components were not included in the Gaia-ESO line list but are listed for eight lines in Table 2 of Scott et al. (2015b, see also their Sect. 6.8.2). Five of these lines are among our preselected lines, including three lines with both $g f$ flag and synflag $=Y$ (617.681, 622.398, and $637.825 \mathrm{~nm})$. The largest wavelength shift including all isotopes is $0.05 \AA$, while the shifts for the dominating isotopes are $\lesssim 0.03 \AA$. The background line list contains Ni I data from Fuhr et al. (1988) and Wickliffe \& Lawler (1997a), in addition to the sources above, as well as Ni II data from Kurucz (2003).

\section{B.19. Copper $(Z=29)$}

For the six preselected $\mathrm{Cu}$ I lines we adopted the experimental $g f$-values from Kock \& Richter (1968). To be fully consistent with Grevesse et al. (2015) we re-normalised these to the improved life-times of Carlsson et al. (1989), although in terms of $g f$-values the change is less than 0.01 dex. The transition probabilities of these $\mathrm{Cu}$ I lines are rated as accurate $(g f$ flag $=Y)$. One of the six $\mathrm{Cu}$ I lines is considered largely clean $(578.213 \mathrm{~nm}$, synflag $=Y)$ for abundance purposes, while the others are blended to varying degrees $($ synflag $=U$ ). For $\mathrm{Cu}$ I lines only appearing in the background line list we adopted transition probabilities from Kurucz (2012), as well as from Biémont (1973) for a few lines. In the Solar System $\mathrm{Cu}$ consists to more than two thirds of ${ }^{63} \mathrm{Cu}$ and almost one third ${ }^{65} \mathrm{Cu}$, both of which have non-zero nuclear spin and therefore cause hyperfine splitting (see Table 3). Table C.5 provides the adopted data for HFS and isotope shifts.

\section{B.20. $\operatorname{Zinc}(Z=30)$}

For the two preselected $\mathrm{Zn}$ I lines at 481.053 and $636.234 \mathrm{~nm}$ the $g f$-values were taken from the theoretical work of Biemont \& Godefroid (1980). In the case of $481.053 \mathrm{~nm}$ this was further slightly re-normalised to the more accurate life-times measured 
U. Heiter et al.: Atomic data for the Gaia-ESO Survey
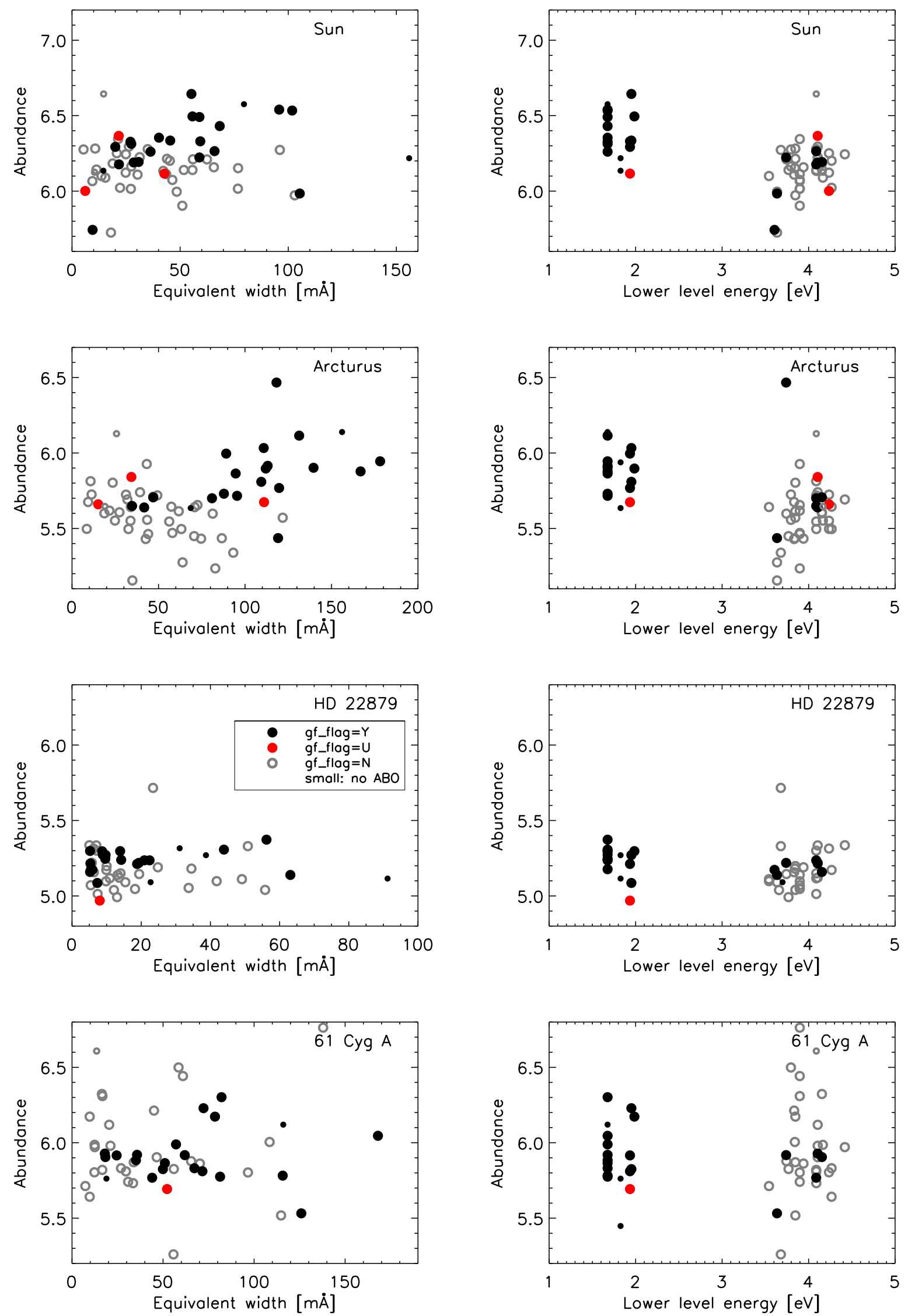

Fig. B.13. Line-by-line Ni I abundances determined for four benchmark stars for three groups of lines with different quality assessment of their transition probabilities, as a function of equivalent width and lower level energy. Abundances are given as $\log \left(\varepsilon_{\mathrm{Ni}}\right)+12$, where $\varepsilon_{\mathrm{Ni}}=N_{\mathrm{Ni}} / N_{\mathrm{H}}$. Only lines with synflag $=Y$ or $U$ and with equivalent widths $>5 \mathrm{~m} \AA$ are included. 
by Kerkhoff et al. (1980) as in Grevesse et al. (2015). These transition probabilities are rated reliable $(g f$ flag $=Y)$, but the lines are partly blended (synflag $=U$ ), including $636.234 \mathrm{~nm}$ which is located in a Ca I autoionising line. The isotopes ${ }^{64} \mathrm{Zn},{ }^{66} \mathrm{Zn}$, and ${ }^{68} \mathrm{Zn}$ all contribute significantly to the Solar System abundance of $\mathrm{Zn}$ (see Table 3), but data for isotopic shifts are not available. Hyperfine splitting is of little importance owing to the low natural abundance of the single isotope of $\mathrm{Zn}$ with non-zero spin.

\section{B.21. Strontium $(Z=38)$}

Oscillator strengths for the preselected $\mathrm{Sr}$ I lines were taken from García \& Campos (1988) with $g f_{\text {flag }}=Y$ (five lines), Werij et al. (1992) and Vaeck et al. (1988) with $g f$ flag $=U$, and Corliss \& Bozman (1962) with $g f \_l a g=N$. García \& Campos (1988) measured relative transition probabilities from emission-line spectrometry in a hollow cathode discharge lamp. These were placed on an absolute scale using previously published experimental life-times by other authors. The data from Werij et al. (1992) are derived from a combination of R-matrix multichannelquantum-defect-theory and modified Coulomb-approximation calculations, complemented by branching-ratio measurements.

None of the preselected lines are blend-free in the Sun or Arcturus, and there are only two lines with synflag $=U$ $(679.102 \mathrm{~nm}$ with $g f$ flag $=Y$ and $654.678 \mathrm{~nm}$ with $g f$ flag $=U)$. Both of these are very weak, and the remaining eight Sr I lines have synflag $=N$. The background line list also contains a few Sr II lines, with data from Warner (1968).

Strontium has three stable isotopes, one of them with nonzero spin (see Table 3). Owing to the low Solar System abundance of the latter, we expect the effect of HFS to be negligible for Sr. Hughes (1957) presented measurements of isotope shifts for $13 \mathrm{Sr}$ I lines within the Gaia-ESO wavelength range. All of them have negligible shifts $(\lesssim 2 \mathrm{m \AA})$.

\section{B.22. Yttrium $(Z=39)$}

For the few Y I lines in the preselected line list only the semiempirical calculations by Kurucz (2006) are available, which were adopted for this work. Because the accuracy of these values is not known they were assigned a $g f \_f a g=N$. All of these lines are furthermore blended to some degree in the Sun and Arcturus (see Table 1).

The situation is much better for the more numerous Y II lines, all of which are contained in the recent experimental work by Biémont et al. (2011) and were assigned a $g f$ flag $=Y$. Four of these lines are also unblended in the Sun and Arcturus (synflag $=Y ; 488.368,508.742$, 528.982, $572.889 \mathrm{~nm})$, and seven additional lines have uncertain blend status (synflag $=U$ ). The background line list also contains data from Kurucz (2011) for Y II.

Yttrium has only one stable isotope, which has non-zero spin (see Table 3). HFS data are listed in Table 2 of Grevesse et al. (2015, see also their Sect. 4.8) for seven of our preselected Y II lines. However, these data result in HFS components with negligible wavelength difference $(\lesssim 5 \mathrm{m \AA})$.

\section{B.23. Zirconium $(Z=40)$}

For the preselected ZrI lines we adopted the $g f$-values of Biemont et al. (1981), who measured life-times and branching ratios by laser-induced fluorescence and emission spectrometry. For two of the three preselected $\mathrm{Zr}$ II lines we used the experimental transition probabilities of Ljung et al. (2006, $g f$ flag $=Y$ ), and for the third line the estimated oscillator strength of Cowley $\&$ Corliss $(1983, g f f l a g=U$ ) was used. In addition to these sources the background line list contains $\mathrm{Zr}$ I data from Corliss \& Bozman (1962) and oscillator strengths estimated by R. L. Kurucz from multiplet table intensities for $\mathrm{Zr}$ I and $\mathrm{Zr}$ II lines.

The $\mathrm{Zr}$ lines are all very weak in solar-type stars but can be quite strong in cooler stars. Two examples are shown in Fig. B.14. Thus, a detailed blending analysis and line selection should be done whenever stars are analysed whose parameters differ from those of the Sun and Arcturus. Five of the $\mathrm{Zr}$ I lines seem to be good candidates for abundance analysis and were assigned synflag $=Y$, with the remainder having synflag $=U$. The three Zr II lines all seem to be affected by blends (see Table 1).

Zirconium has five stable isotopes which all contribute significantly to the Solar System abundance (see Table 3). Three of them contribute at the $20 \%$ level or more, and all of these have zero spin. Therefore we did not include any HFS components ${ }^{25}$. Isotopic shifts were measured by Langlois \& Gagné (1994) for $330 \mathrm{Zr}$ I lines between 536 and $706 \mathrm{~nm}$. The maximum shift between ${ }^{90} \mathrm{Zr}$ and ${ }^{94} \mathrm{Zr}$ in their list was $8 \mathrm{~m} \AA$. Similar values have been reported by Bourauel et al. (1987) and Lim et al. (1998). This level of isotopic splitting is negligible in the context of the GES.

\section{B.24. Niobium $(Z=41)$}

Accurate experimental oscillator strengths for the eight preselected $\mathrm{Nb}$ I lines were measured by Duquette et al. (1986). In addition, the background line list contains $\mathrm{Nb}$ I data from older measurements of Corliss \& Bozman (1962) and Duquette \& Lawler (1982). Niobium has one isotope with a nuclear spin of $9 / 2$ and can therefore be expected to show hyperfine structure. Because the $\mathrm{Nb}$ lines are all weak, HFS splitting has not been accounted for, but relevant data can be found in Nilsson \& Ivarsson (2008) and Nilsson et al. (2010). All of the Nb I lines are heavily blended $($ synflag $=N)$.

\section{B.25. Molybdenum $(Z=42)$}

Experimental transition probabilities for the six preselected Mo I lines are available from Whaling \& Brault (1988), who used measured radiative life-times and branching ratios. These are rated accurate $\left(g f \_l a g=Y\right)$. The Mo lines are all very weak, and therefore hyperfine and isotope splitting have not been accounted for. The primary Mo abundance indicators are 575.141 and $603.064 \mathrm{~nm}$ (synflag $=Y$ ), although they are too weak in dwarfs to be useful (see Fig. B.15).

\section{B.26. Ruthenium $(Z=44)$}

We included one line of $\mathrm{Ru} \mathrm{I}$ in the preselected line list $(486.9 \mathrm{~nm})$ with an experimental (life-time and branching ratio) $g f$-value from Wickliffe et al. (1994). The line is not included in the more recent work of Fivet et al. (2009). The adopted $g f$-value is considered accurate $\left(g f \_l a g=Y\right)$, but the line is significantly blended (synflag $=U$ ). In addition, the background line list contains Ru I data from Corliss \& Bozman (1962), Biemont et al. (1984), Salih \& Lawler (1985), and R. L. Kurucz (based on multiplet tables). The Ru lines are all very weak and therefore hyperfine and isotope splitting have not been accounted for.

25 HFS $A$ and $B$ constants can be found for 12 levels of $\mathrm{Zr} \mathrm{I}$ in Lim et al. 1998 , which correspond to the lower levels of some of our preselected lines. 

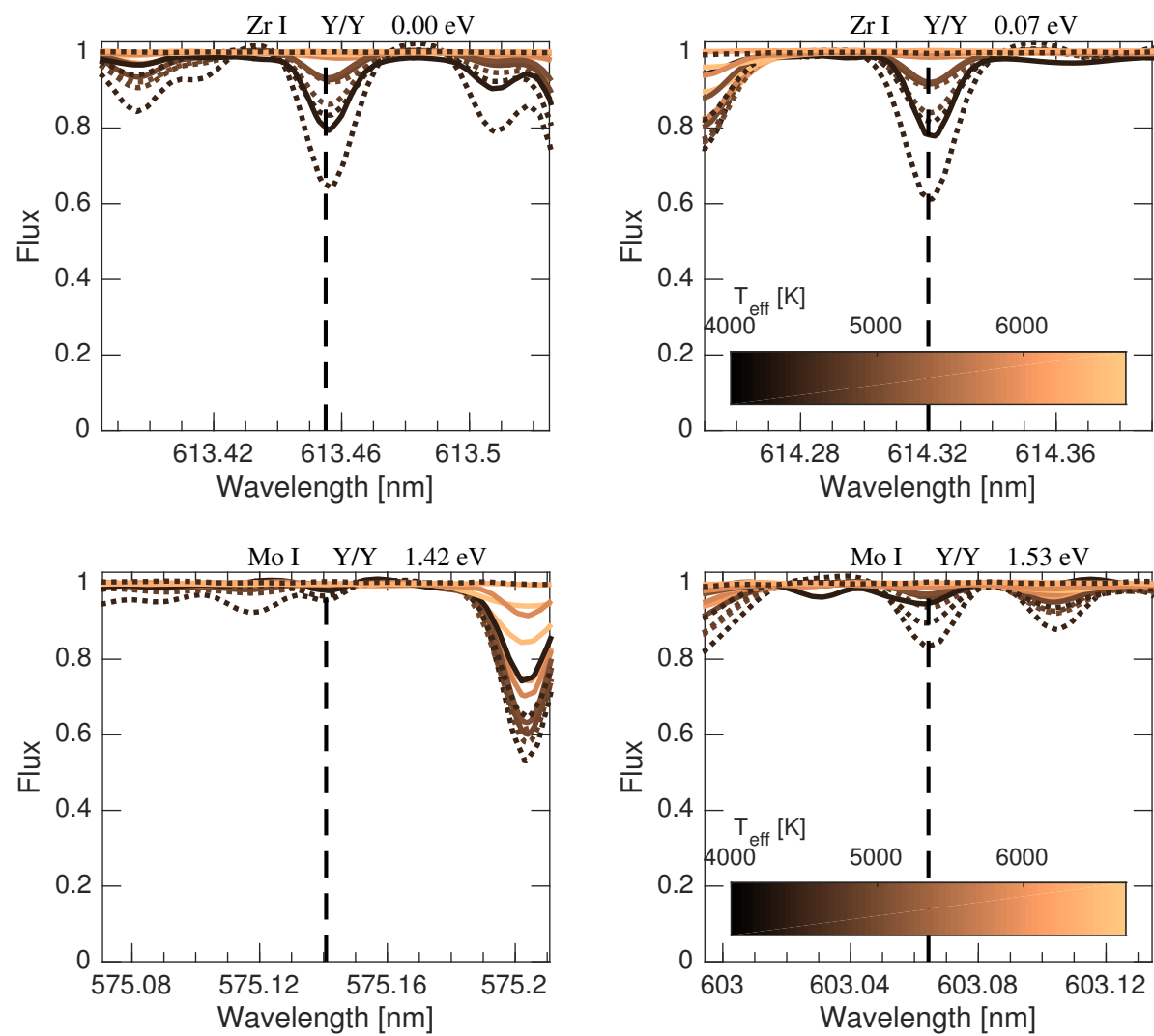

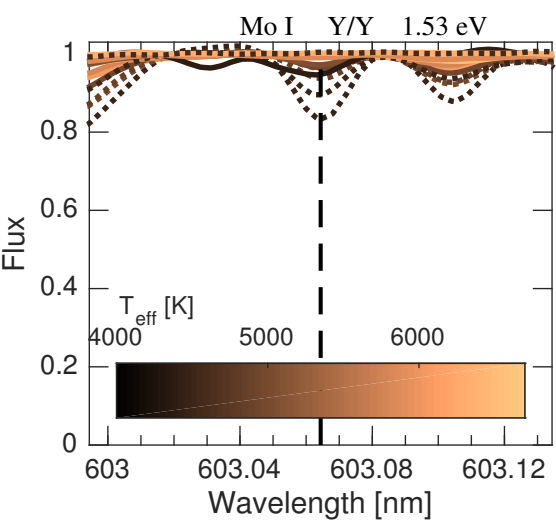

Fig. B.14. Line profiles for two high-quality preselected $\mathrm{Zr}$ I lines, which are weak in solartype stars but strong in cooler stars. Generated from observed spectra of selected Gaia FGK benchmark stars. See Sect. 2.3.

Fig. B.15. Line profiles for high-quality preselected Mo I lines generated from observed spectra of selected Gaia FGK benchmark stars. See Sect. 2.3.

\section{B.27. Barium $(Z=56)$}

We used the experimental data from Davidson et al. (1992) for all four preselected Ba II lines. Two of the lines are also included in the experimental work of Kurz et al. (2008). The $\log g f$ values from Kurz et al. (2008) are lower than those from Davidson et al. (1992) by $\sim 0.1$ dex. For consistency, we adopted the source containing all four lines. All four lines are strong in stellar spectra except for the most metal-poor stars. The line at $493.408 \mathrm{~nm}$ is strongly blended in all but the most metal-poor stars (see Fig. B.16). In addition, the background line list contains Ba I data from Corliss \& Bozman (1962) and data from Miles \& Wiese (1969) for Ba I and Ba II lines.

HFS and IS data. Solar System material contains a mixture of several stable Ba isotopes, five of which contribute with more than $1 \%$ to the $\mathrm{Ba}$ abundance (Table 3). Isotopic splittings have been measured for the $493.408 \mathrm{~nm}$ line by Wendt et al. (1984), for the 585.367 and $614.171 \mathrm{~nm}$ lines by Van Hove et al. (1982), and for the $649.690 \mathrm{~nm}$ line by Villemoes et al. (1993). The wavelength shifts are summarised in a table in the online database of R.L. Kurucz ${ }^{26}$ and the largest ones are $2 \mathrm{m \AA}$ (between isotopes 134 and 138). Although this can be considered to have a negligible effect in the context of the GES and similar surveys, all five isotopic components were included for the preselected Ba II lines.

Two of the isotopes have non-zero nuclear spin leading to HFS in BaII lines with non-negligible effects on abundance analysis. Experimental determinations of the HFS $A$ and $B$ constants were found for all levels involved in the preselected Ba II lines in Becker et al. (1981), Silverans et al. (1986), and

${ }^{26}$ http://kurucz .harvard.edu/atoms/5601/isoshifts5601. dat
Villemoes et al. (1993). The complete set of HFS data is given in Table C.6.

\section{B.28. Lanthanum $(Z=57)$}

We used experimental data from Lawler et al. (2001a) for five of the preselected La II lines $\left(g f \_l a g=Y\right)$. For the sixth line at $593.6 \mathrm{~nm}$ the only experimental data available are from Corliss \& Bozman (1962), which is known to have large, systematic uncertainties (see e.g. Arnesen et al. 1977; Wahlgren 2010). We did not attempt to apply any corrections to the Corliss \& Bozman (1962) data or adopt any previous such attempts. Instead, we resorted to the theoretical value from Kułaga-Egger \& Migdałek (2009). To assess the uncertainty inherent in that work we compared transition probabilities for lines in common with Lawler et al. (2001a), as shown in Fig. B.17. The comparison shows a similar scatter as the comparison between the Lawler et al. (2001a) data and data from Bord et al. (1996, see Figs. 1 to 3 in Lawler et al. 2001a), which are based on a semi-empirical calibration of data from Meggers et al. (1975). We assigned a $g f$ flag $=U$ to the $593.6 \mathrm{~nm}$ line. In addition, the background line list contains data for La I and La II by Corliss \& Bozman (1962) and for La II by Zhiguo et al. (1999).

Most of the preselected lines are blended to different degrees in the spectra of the Sun, Arcturus, and other benchmark stars (see Figs. B.18 and B.19). The line at $492.2 \mathrm{~nm}$ appears rather clean in observed spectra, but a synthesis including surrounding lines from the background line list yields a significantly stronger line than a synthesis for the La II line only. A look into the background line list reveals several lines of different species with similar strength at the same wavelength ( $\mathrm{Si}$ I, Ti I, Ce II, Y I). Hence, the line is not recommended for abundance analysis (synflag $=N)$. The only line with synflag $=Y$ at $593.6 \mathrm{~nm}$ is invisible 

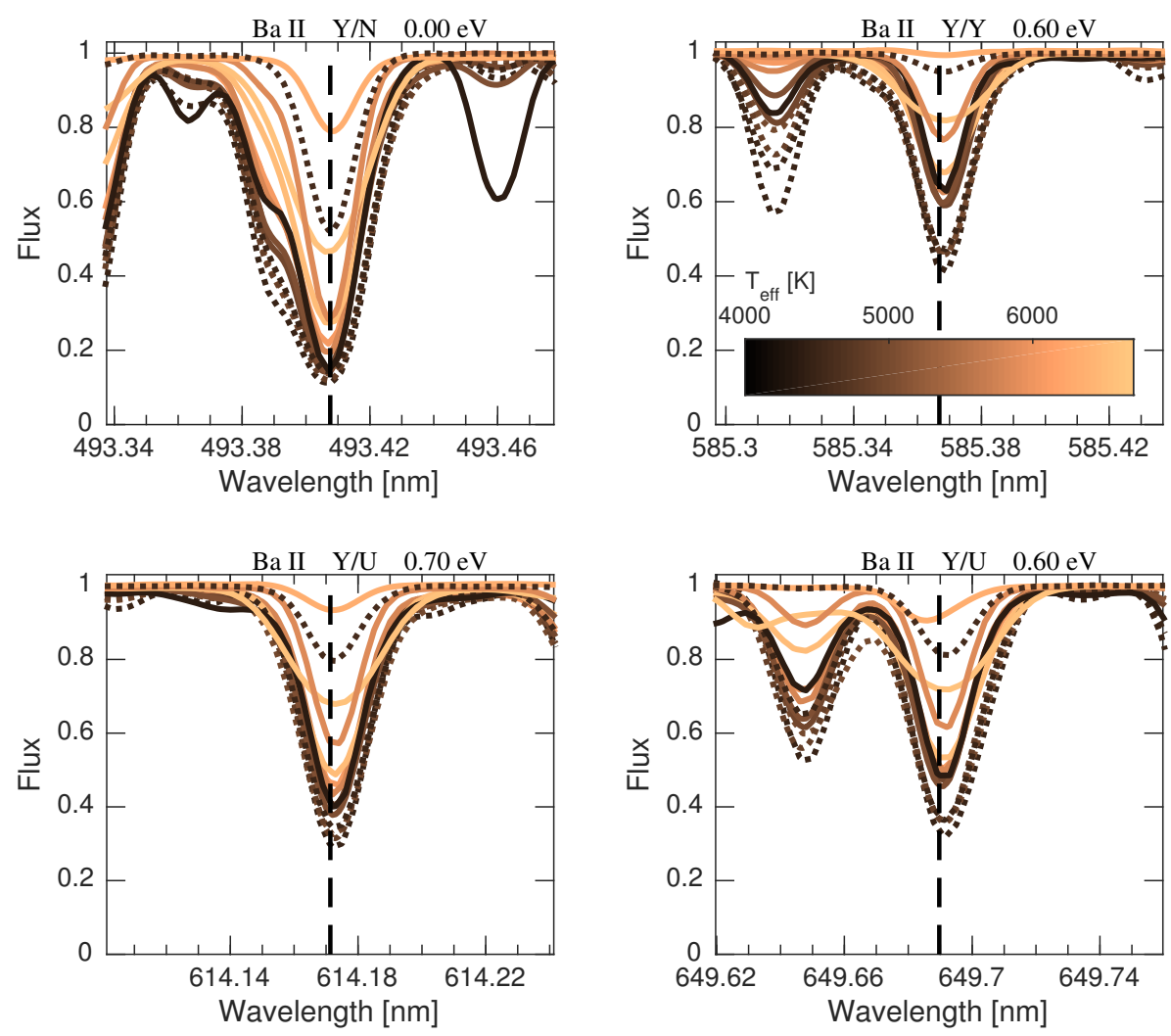

Fig. B.16. Line profiles for preselected Ba II lines generated from observed spectra of selected Gaia FGK benchmark stars. See Sect. 2.3.

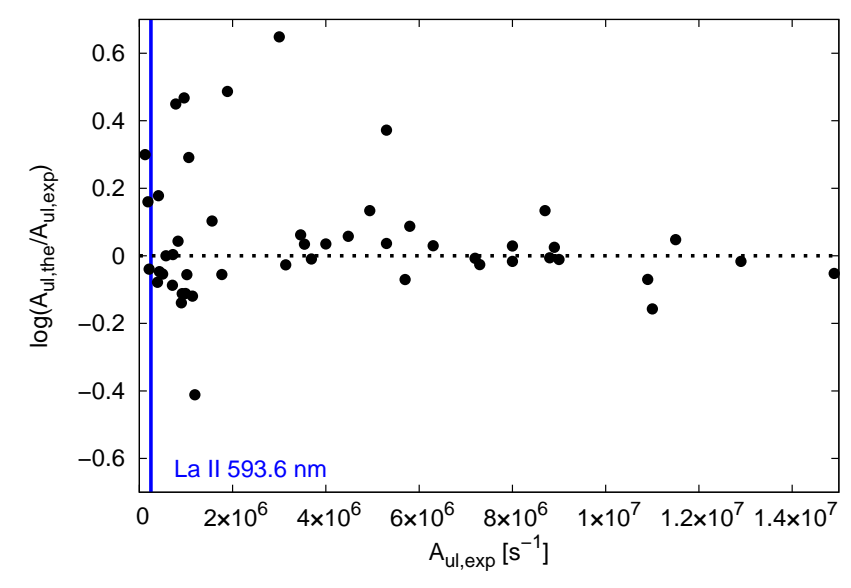

Fig. B.17. Comparison between theoretical (the) and experimental (exp) transition probabilities $A_{\text {ul }}$ for La II. Data are from Kułaga-Egger \& Migdałek (2009) and Lawler et al. (2001a), respectively. The blue vertical line indicates the location of the line at $593.6 \mathrm{~nm}$.

in spectra of dwarf stars and weak in spectra of giant stars but might be stronger in spectra of r-process-rich, metal-poor stars.

HFS data. The single dominant isotope ${ }^{139}$ La makes up 99.9\% of La in Solar System material (Table 3). Thus, La lines will not be affected by isotopic splitting.

On the other hand, HFS should be taken into account for the analysis of La lines. We extracted $A$ and $B$ constants from the literature for each of the nine energy levels involved in the preselected La II transitions, where possible. Most of the data were taken from the experimental work based on ion-beam-laser spectroscopy by Höhle et al. (1982). A similar type of measurement was found for one level in Li et al. (2001). For another two levels HFS constants measured from FTS spectra were found in Lawler et al. (2001a). Semi-empirical calculations of HFS data for the upper level of the line at $492.2 \mathrm{~nm}$ are provided in Furmann et al. (2008), but were not included in the line list. The complete set of HFS data is given in Table C.7.

\section{B.29. Cerium $(Z=58)$}

High-quality experimental data for Ce II lines from BFs and lifetimes are available from Lawler et al. $(2009, g f$ flag $=Y$ ) for two thirds of the 12 preselected lines. For the other lines we used theoretical data from Palmeri et al. $(2000, g f$ flag $=U)$. The latter used two different approaches to calculate radiative life-times, designated "Calculation A" and "Calculation B". According to the authors, Calculation $\mathrm{B}$ is expected to be more accurate than Calculation A. Palmeri et al. (2000) give $g f$-values using Calculation B for selected lines, including one of the lines considered here $(511.7 \mathrm{~nm})$. The background line list contains numerous additional Ce II lines with data from these two sources, as well as Ce I lines with data from Corliss \& Bozman (1962) and Cowley (1973).

The values for the three preselected NIR lines are taken from the online DREAM database ${ }^{27}$, which appears to provide the results of Calculation A of Palmeri et al. (2000). In addition to having uncertain $g f$-values, none of these three lines is recommended for abundance analysis due to their unfavourable blending properties ( synflag $=U$ or $N)$.

Among the optical lines two appear unblended in the spectra of the Sun, Arcturus, and other benchmark stars (Fig. B.20, lower panels), while two others are strongly blended and cannot be recommended for abundance analysis (Fig. B.20, upper panels). The remaining lines were assigned a synflag $=U$, and their usage needs to be decided on a case-by-case basis.

\footnotetext{
${ }^{27}$ http://hosting.umons.ac.be/html/agif/databases/ dream.html
} 

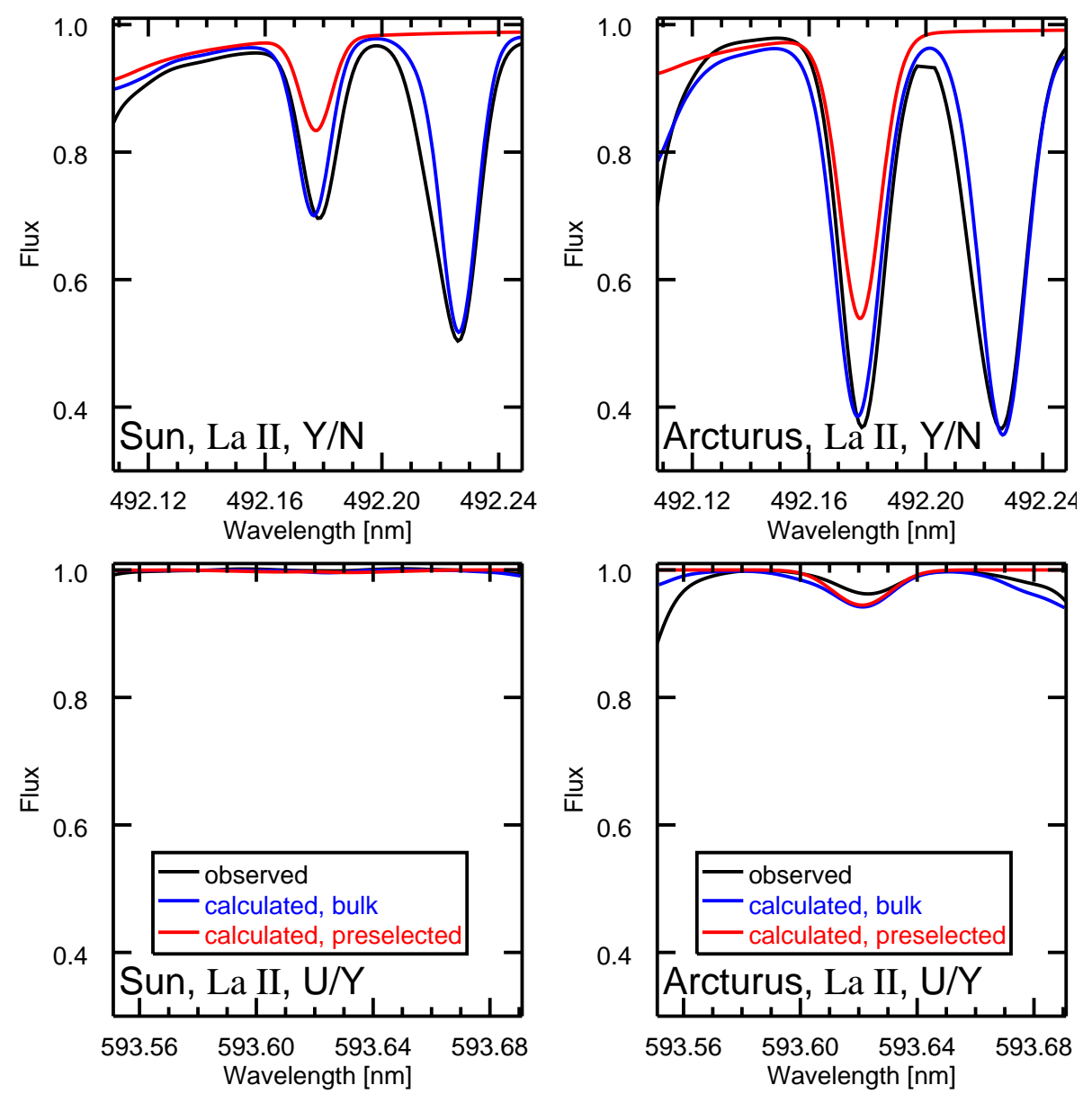

Fig. B.18. Comparison of observed and calculated line profiles around the two La II lines at $492.2 \mathrm{~nm}$ (upper panels, gf_flag $=Y$, synflag $=N$ ), and at $593.6 \mathrm{~nm}$ (lower panels, gf flag $=U$, synflag $=Y$ ) for the Sun (left) and Arcturus (right). Black lines: observations, red lines: calculations including preselected spectral lines only, blue lines: calculations including blends from background line list.

Cerium has four stable isotopes, all with zero nuclear spin. Two of them contribute to the Solar System abundance with more than $1 \%$ (Table 3). However, the isotope splitting is negligible for Ce II lines (Lawler et al. 2009).

\section{B.30. Praseodymium $(Z=59)$}

Experimental $g f$-values are available for all seven preselected Pr II lines from Ivarsson et al. (2001, six lines) and from Li et al. (2007a, four lines), where three lines are in common. In general, the data from the two publications agree well (see Fig. 2 of Sneden et al. 2009). An exception is the line at $532.3 \mathrm{~nm}(0.2 \mathrm{dex}$ difference in $\log g f$ ). Sneden et al. (2009) found that the higher value from $\mathrm{Li}$ et al. (2007a) results in a more consistent solar $\mathrm{Pr}$ abundance. Synthetic spectra calculated for Arcturus with scaled solar abundances and the Li et al. (2007a) $g f$-value also support the validity of the higher value (see Fig. B.21). In summary, we used the data from Ivarsson et al. (2001) for five lines, and data from Li et al. (2007a) for two lines $(521.7 \mathrm{~nm}$ and $532.3 \mathrm{~nm})$, all with $g f$ flag $=Y$.

We note that the $g f$-values given in Table 2 of Li et al. (2007a) do not agree with the values obtained by using their Eq. (5), with differences reaching more than 0.005 dex in some cases. We used the calculated values instead of the tabulated ones (the differences calculated minus tabulated are $+0.017 \mathrm{dex}$ and -0.018 dex for the two lines, respectively). In addition to the sources above, the background line list contains Pr II data from Biémont et al. (2003), data from Meggers et al. (1975) for Pr I and Pr II lines, and data from Ryabtsev (priv. comm.) for a few Pr III lines.
Most of the preselected PrII lines are invisible and/or severely blended in the spectra of the Sun, Arcturus, and other benchmark stars. However, two lines at 526.0 and $532.3 \mathrm{~nm}$ have non-negligible strengths and seem to be more or less unblended (synflag $=U$ and $Y$, respectively, see Fig. B.21). Five of the lines (the previous two and 517.4, 522.0, $529.3 \mathrm{~nm}$ ) were used by Sneden et al. (2009) to derive the Pr abundance in r-processrich, metal-poor $([\mathrm{Fe} / \mathrm{H}] \approx-3 \mathrm{dex})$ stars, in which these lines are stronger than in the Sun.

HFS data. Praseodymium has a single stable isotope, ${ }^{141} \mathrm{Pr}$, with non-zero nuclear spin, giving rise to HFS in Pr II lines. For each of the 12 energy levels involved in the preselected Pr II transitions HFS $A$ constants are available in the literature. Electric quadrupole interaction is considered negligible for the HFS of Pr (e.g. Ginibre 1989; Sneden et al. 2009), and thus the HFS $B$ constants were set to zero for all levels. Five of the levels are included in the experimental work based on fast-ion-beam laser spectroscopy by Rivest et al. (2002). Data for the remaining levels were taken from the work of Ginibre (1989), who determined HFS $A$ constants from an analysis of FTS spectra. The complete set of HFS data is given in Table C.8.

\section{B.31. Neodymium $(Z=60)$}

With over 50 lines Nd II lines are the most numerous among all rare earth species in the preselected line list. For the majority of these lines, including one of the four lines in the NIR, we used the experimental $g f$-values determined by Den Hartog et al. $(2003$, gf_flag $=Y)$ from a combination of LIF measurements 

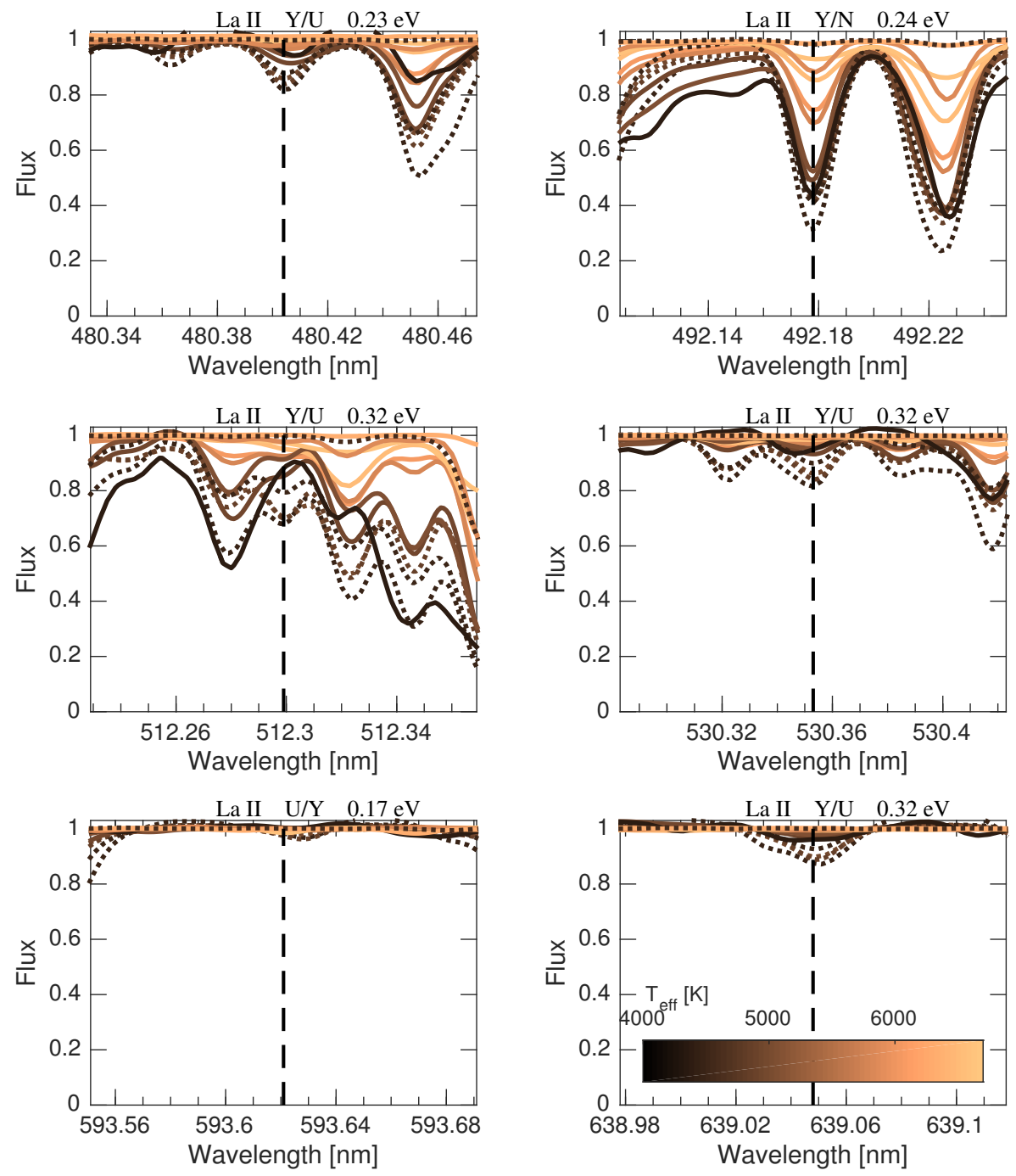

Fig. B.19. Line profiles for preselected La II lines generated from observed spectra of selected Gaia FGK benchmark stars. See Sect. 2.3.

of radiative life-times and FTS measurements of BFs. The $g f$-values for the remaining lines (reference code MC in the CDS table) are from Kurucz \& Bell (1995). They are derived from a calibration of measured line intensities from Meggers et al. (1975) following the procedure outlined by Cowley \& Corliss (1983, T.A. Ryabchikova and R.L. Kurucz, priv. comm.). A comparison of the MC-derived data to the Den Hartog et al. (2003) data for 133 lines in common within the UVES-580 wavelength range is shown in Fig. B.22. The comparison is characterised by a standard deviation of $0.22 \mathrm{dex}$ and additional systematic differences varying with wavelength. We assigned a $g f$ flag $=U$ to the $g f$-values from this source.

For five lines with MC-derived data there are also experimental $g f$-values by $\mathrm{Li}$ et al. (2007b) available (which are between 0.04 dex larger and 0.16 dex smaller), but these were not included in the line list. In addition, the background line list contains data from Meggers et al. (1975) for Nd I lines, from $\mathrm{Xu}$ et al. (2003a) for Nd II lines, and from Ryabchikova et al. (2006) for Nd III lines.

The four preselected Nd II lines in the NIR are invisible in solar-like and giant stars, with the possible exception of one line in metal-rich giants such as $\mu$ Leo. Only two of the lines in the UVES-580 range appear unblended in solar- or Arcturus-like stars $($ synflag $=Y)$. However, their $g f$-values are uncertain, and at least one of them could be affected by unidentified blends. About half of the remaining lines are clearly blended $($ synflag $=N)$, and the other half are border-line cases or lines for which the blending properties strongly depend on stellar parameters $($ synflag $=U)$. A few selected cases are illustrated in Figs. B.23 and B.24. We note that this assessment is not directly applicable to r-process-rich, metal-poor stars, for which the relative strengths of $\mathrm{Nd}$ II lines and blending lines might be significantly different.

HFS and IS data. Neodymium has seven stable isotopes with rather evenly distributed Solar System abundance ratios (Table 3). Data for IS are available for all of the preselected Nd II lines for the isotope pair 144-150 and for 41 of the lines for the isotope pair 142-144. For the remaining isotopes the wavelengths were set to those of isotope 150 . The shifts in level energy for the 142-144 pair were measured by Ahmad \& Saksena (1981) on hollow cathode lamp spectra recorded by a Fabry-Perot spectrometer. For the 144-150 pair most of the required levels were measured by Nakhate et al. (1997) using the same technique. Complementary data for the remaining levels were taken from the extensive FTS work by Blaise et al. (1984). The complete set of IS data used in the Gaia-ESO line list is given in Table C.9.

Koczorowski et al. (2005) measured isotopic shifts for 27 levels of $\mathrm{Nd}$ II for all pairs of $\mathrm{Nd}$ isotopes with even baryon number, using the LIF method (including 13 of the lowest levels and eight of the upper levels of the preselected transitions). Their results are consistent with the sources cited above. The derived 

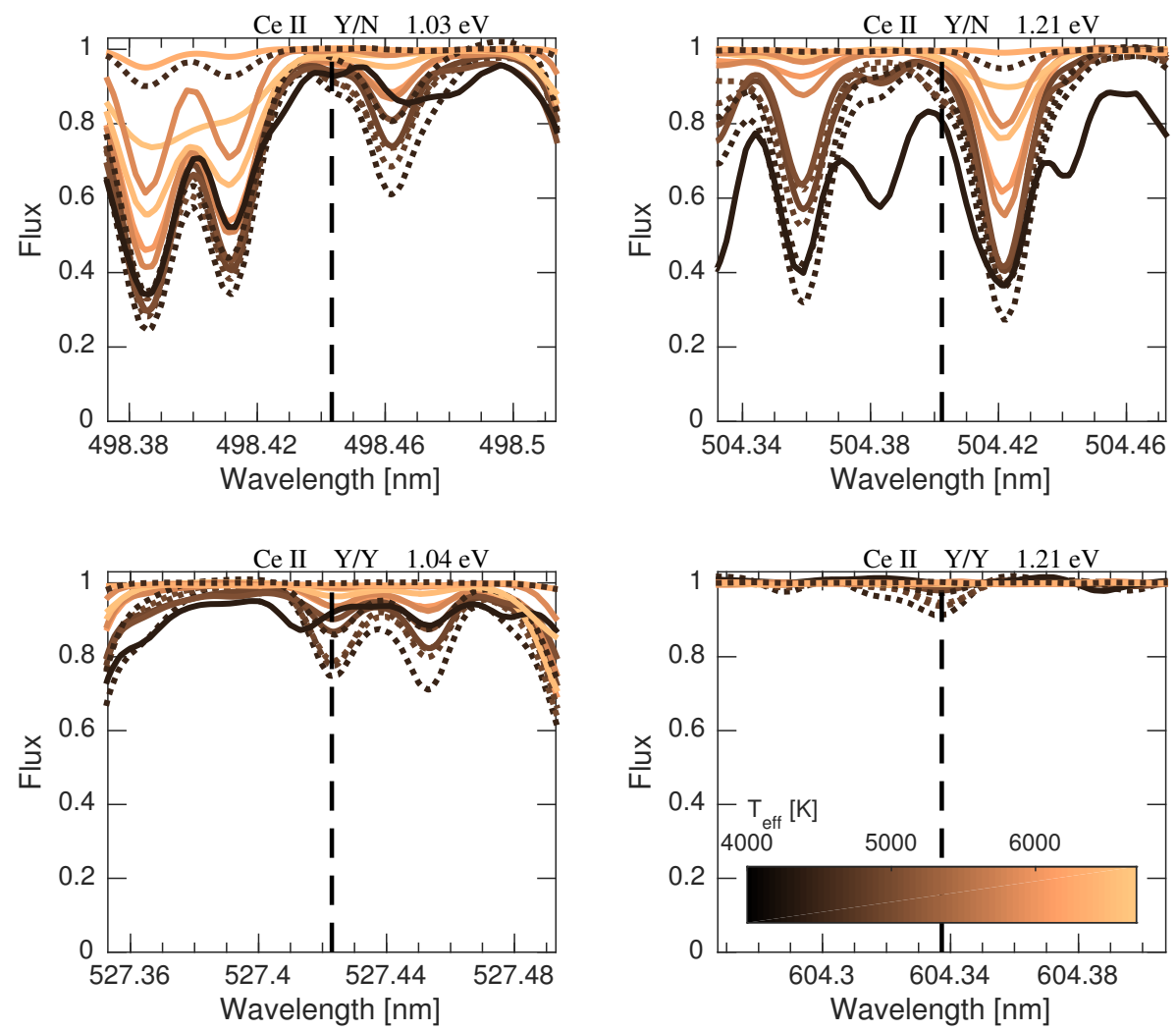

Fig. B.20. Line profiles for preselected Ce II lines generated from observed spectra of selected Gaia FGK benchmark stars. See Sect. 2.3.

shift values are similar for all pairs, although the values for the 150-148 pair tend to be larger than for the others.

Two of the Nd isotopes have odd baryon numbers with non-zero spin and thus hyperfine structure. For 11 even-parity low-energy levels and 64 odd-parity high-energy levels of ${ }^{143} \mathrm{Nd}$ HFS $A$ and $B$ constants were measured by Rosner et al. (2005), using the collinear fast-ion-beam laser spectroscopy technique. For ${ }^{145} \mathrm{Nd} A$ and $B$ constants were then calculated by dividing the constants obtained for ${ }^{143} \mathrm{Nd}$ by the ratios between the nuclear magnetic moments and between the nuclear electric quadrupole moments of ${ }^{143} \mathrm{Nd}$ and ${ }^{145} \mathrm{Nd}$, respectively. These ratios were determined by Rosner et al. (2005) as 1.60884 and 1.92, respectively. These measurements cover 32 out of the $69 \mathrm{Nd}$ II levels involved in the preselected transitions.

For two further lower levels we used data from Ma \& Yang (2004) measured by the same technique. Ma \& Yang (2004) determined $A$ and $B$ constants for both ${ }^{143} \mathrm{Nd}$ and ${ }^{145} \mathrm{Nd}$ for ten levels in total, and their ratios of $A_{143} / A_{145}$ vary between 1.60 and 1.63 , which is in reasonable agreement with the theoretical expectation. Their ratios of $B_{143} / B_{145}$ vary between 1.7 and 2.3 for levels with significant $B$ values, which is comparable to the expected value of 1.9 . However, for two of the levels the $B$ constant changes sign between isotope 143 and 145, including the level at $0.745 \mathrm{eV}$ used here. Together, these sources provide complete HFS data for 16 of the preselected Nd II lines, while about half of the lines have data for only one level, and for a few lines there are no data at all (see Table C.9). The complete set of available HFS data is given in Table C.10.

\section{B.32. Samarium $(Z=62)$}

For the five preselected Sm II lines we used the TRLIF-FTS experimental $g f$-values from Lawler et al. $(2006, g f f l a g=Y$ ). Four of these lines are also contained in the work of Rehse et al. (2006), who used a substantially different experimental method (fast-ion-beam LIF). Their $g f$-values are within 0.05 dex of those by Lawler et al. (2006) for three lines, while their $g f$-value for the $492.956 \mathrm{~nm}$ line is 0.13 dex larger. A detailed comparison between the two studies for over 300 lines in common is given in Lawler et al. (2008a). They found good agreement in general, in particular for the radiative life-time measurements, for which the differences are confined within half of the combined uncertainties quoted by the authors. However, the transition probabilities showed a mean difference of 0.4 times the combined uncertainties and a standard deviation larger than expected from the uncertainties. This led them to conclude that the uncertainties on the BFs from one or both experiments are larger than estimated by the authors. In addition to Lawler et al. (2006) the background line list contains data from Xu et al. (2003b) for Sm II and from Meggers et al. (1975) for Sm I and Sm II lines.

All of the preselected Sm II lines are very weak and affected by blends. The strongest line $(481.6 \mathrm{~nm})$ is severely blended (synflag $=N)$, and the line at $493.0 \mathrm{~nm}$ is invisible in all 'normal' stars. Two examples for lines with synflag $=U$ are shown in Fig. B.25. The one at $485.4 \mathrm{~nm}$ is located in the blue wing of the $\mathrm{H} \beta$ line. Lawler et al. (2006) list further 130 Sm II lines in the UVES-580 wavelength range (and 17 in the GIRAFFE HR21 range), for which a blending analysis could be done, preferably including the parameters of an r-process-rich, metal-poor star.

HFS and IS data. Samarium has seven stable isotopes, each of them contributing significantly to the Solar System abundance of Sm (Table 3). Measurements of IS are not available for any of the preselected Sm II lines. However, isotopic shifts for Sm II lines may usually be neglected compared to other line broadening mechanisms. For example, the largest shift between ${ }^{147} \mathrm{Sm}$ and ${ }^{154} \mathrm{Sm}$ given by Masterman et al. (2003) for 87 lines with wavelengths between 418 and $465 \mathrm{~nm}$ is $0.05 \AA$ (somewhat larger shifts were measured for the rare isotope ${ }^{144} \mathrm{Sm}$ ). Nevertheless, individual lines for each of the seven isotopes are 

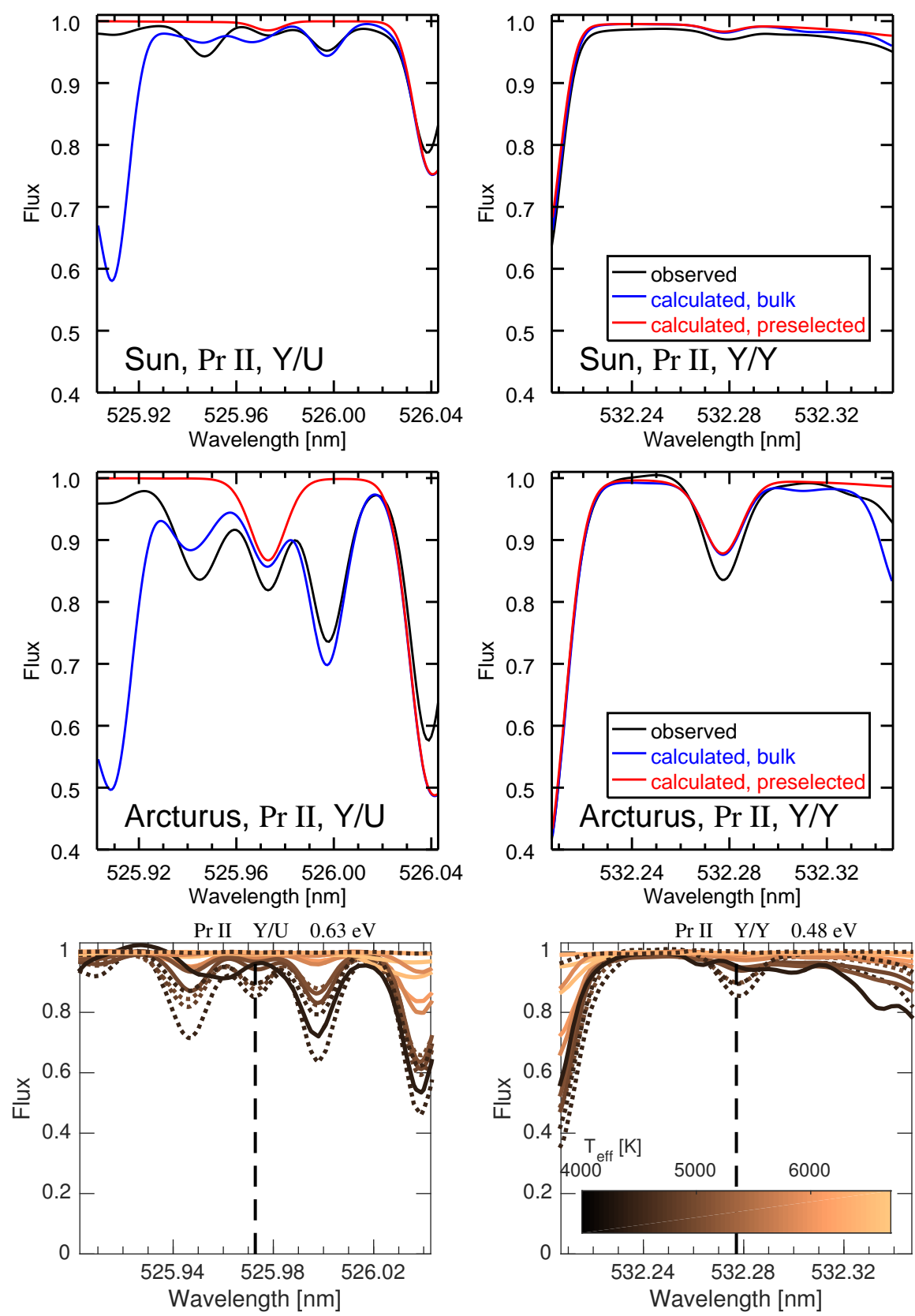

Fig. B.21. Observed and calculated line profiles around two preselected Pr II lines for the Sun (top row) and Arcturus (middle row). Black lines: observations, red lines: calculations including preselected spectral lines only, blue lines: calculations including blends from background line list. Bottom row: line profiles generated from observed spectra of selected Gaia FGK benchmark stars. See Sect. 2.3.

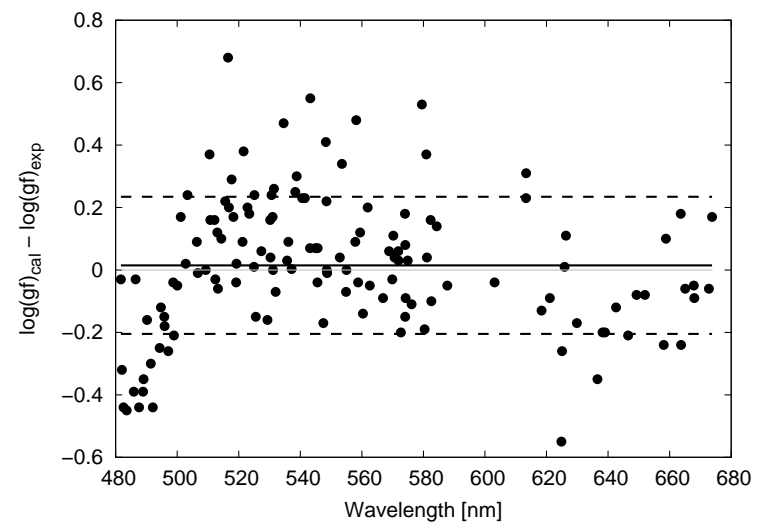

Fig. B.22. Comparison between calibrated (cal) and experimental (exp) transition probabilities for Nd II lines in the UVES-580 wavelength range. The data are from Kurucz \& Bell (1995) based on Meggers et al. (1975) and from Den Hartog et al. (2003), respectively. The solid black line indicates the mean difference in $\log g f$, and the dashed lines indicate the standard deviation.

A106, page 46 of 63 included in the line list (with equal wavelengths and $g f$-values for those without HFS), to allow the user to apply scaling according to isotopic abundances.

For the two Sm isotopes with non-zero spin HFS $A$ and $B$ constants are available from the experimental work by Masterman et al. (2003) based on collinear fast-ion-beam laser spectroscopy. Their data cover all of the ten levels involved in the preselected Sm II transitions except one (the upper level of the line at $483.7 \mathrm{~nm}$ ) and are summarised in Table C.11. We note that Masterman et al. (2003) write that they fixed the ratios of $A_{147} / A_{149}$ and $B_{147} / B_{149}$ for each level to the values corresponding to the ratios of the nuclear magnetic and electric quadrupole moments (1.213 and -3.5), respectively. This does not appear to be the case for the $B$ constants, however, for which the ratios for the levels of interest vary between -3.4 and -3.7 .

\section{B.33. Europium $(Z=63)$}

For the preselected Eu II lines we rely on the $g f$-values measured by Lawler et al. (2001c) based on experimental life-times and 

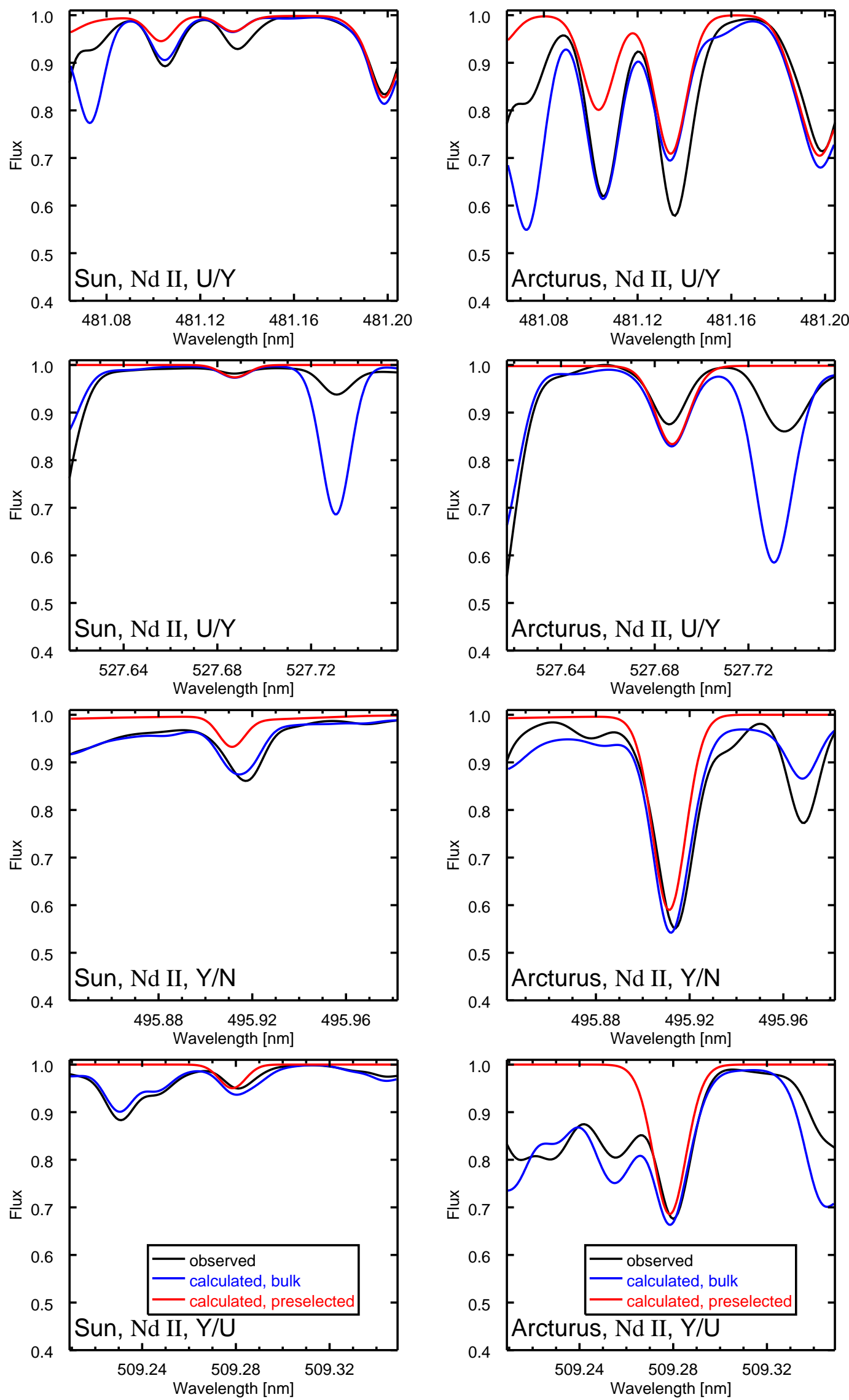

Fig. B.23. Comparison of observed and calculated line profiles around four of the preselected Nd II lines for the Sun (left) and Arcturus (right). Black lines: observations, red lines: calculations including preselected spectral lines only, blue lines: calculations including blends from background line list. 

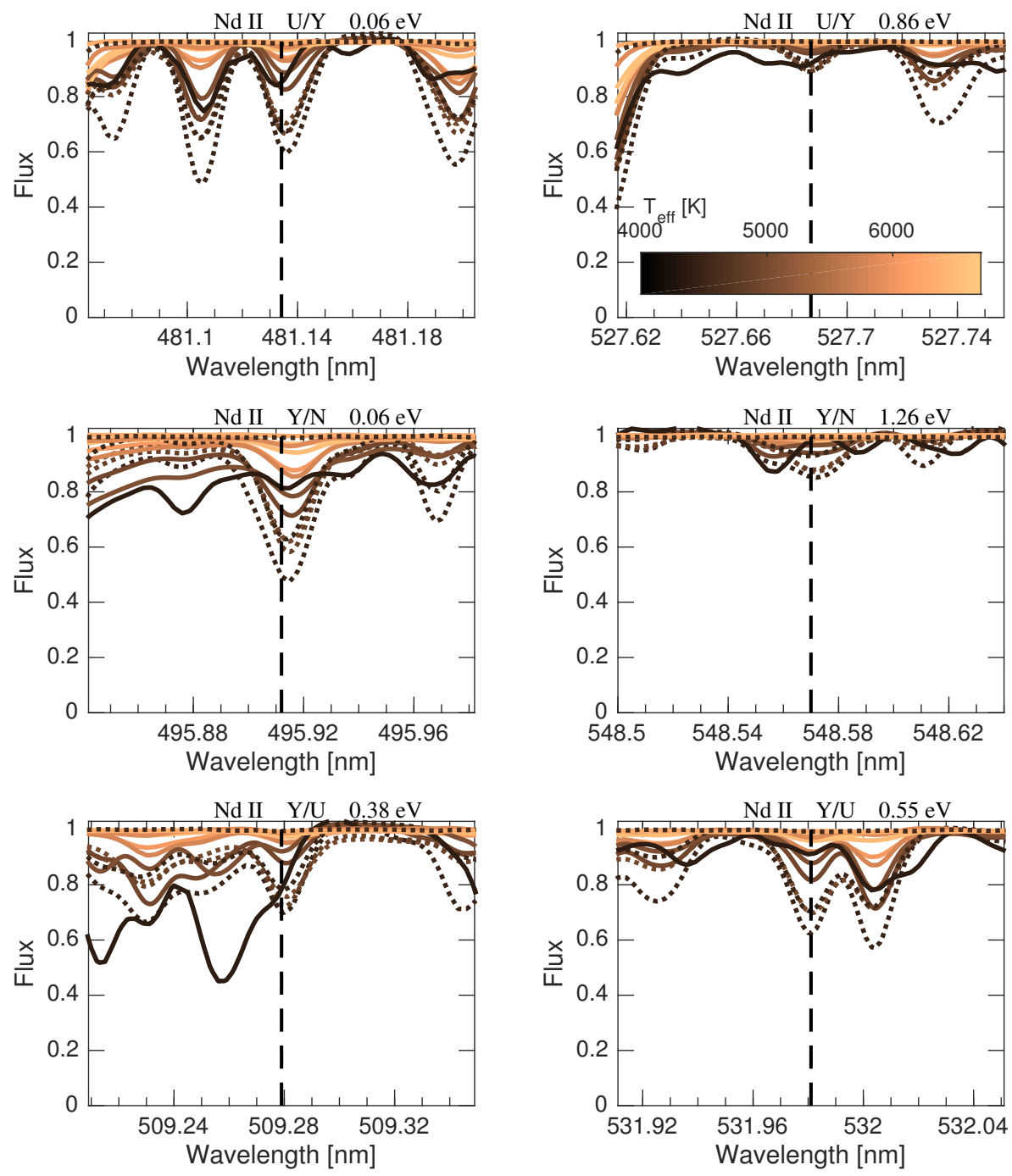

Fig. B.24. Line profiles for preselected Nd II lines generated from observed spectra of selected Gaia FGK benchmark stars. See Sect. 2.3.

BFs. In addition, the background line list contains data by Den Hartog et al. (2002) for Eu I, by Zhiguo et al. (2000) for Eu II, and by Meggers et al. (1975) for both ions.

The five preselected Eu II lines are rather weak and partly blended in the spectra of the Sun, Arcturus, and other benchmark stars. Figure B.26 shows the line profiles of the two strongest lines, of which the one at $643.8 \mathrm{~nm}$ is clearly blended $($ synflag $=N)$. The line at $664.5 \mathrm{~nm}$ might be useful for giant stars at high signal-to-noise ratios (to be decided on a case-by-case basis, synflag $=U$ ).

HFS and IS data. Europium occurs in the form of two isotopes $\left({ }^{151} \mathrm{Eu}\right.$ and ${ }^{153} \mathrm{Eu}$, with non-zero nuclear spin), each contributing about equally to the Solar System Eu abundance (Table 3). Isotopic shifts for 24 optical Eu II transitions were compiled by Lawler et al. (2001c, their Table 5). These are in general smaller than for Sm II lines and particularly small for the preselected Eu II lines $(\lesssim 0.02 \AA$ A, not resolved in FTS spectra). Therefore, IS data were not included for Eu II lines in the Gaia-ESO line list.

For four energy levels involved in the preselected Eu II transitions we used the experimental HFS $A$ and $B$ constants by Hühnermann et al. (1992) and Möller et al. (1993), respectively. These are based on the LIF method and are in excellent agreement with the measurements by Sen \& Childs (1987) obtained from laser radio frequency double resonance spectroscopy (see compilation by Lawler et al. 2001c, their Table 4). For the remaining three levels we used the LIF data from Villemoes et al. (1992), who give essentially the same values as Hühnermann et al. (1992) and Möller et al. (1993) for these levels. The complete set of HFS data is given in Table C.12.

\section{B.34. Gadolinium $(Z=64)$ and Dysprosium $(Z=66)$}

Gd II and Dy II are the heaviest species included in the preselected line list, with one line each (at 486.504 and $516.969 \mathrm{~nm}$, respectively). Laboratory transition probabilities based on the TRLIF-FTS technique have been published for the two lines by Den Hartog et al. (2006) and Wickliffe et al. (2000), respectively, where the latter provide data for Dy I lines as well. In addition to these sources, the background line list contains data for Gd I, Gd II, Dy I, and Dy II from Meggers et al. (1975).

Both of the preselected lines are very weak and blended in the spectra of the Sun, Arcturus, and other benchmark stars and thus are of questionable use for a meaningful abundance analysis (synflag $=U$ ). In dwarf stars the GdII line is located in the red wing of the $\mathrm{H} \beta$ line. The line profiles for both transitions are illustrated in Fig. B.27. However, Den Hartog et al. (2006) and Wickliffe et al. (2000) list about 150 and 30 Gd II and Dy II lines, respectively, in the UVES-580 wavelength range (and about ten lines each in the GIRAFFE HR21 range), for which a blending analysis could be done. This analysis 

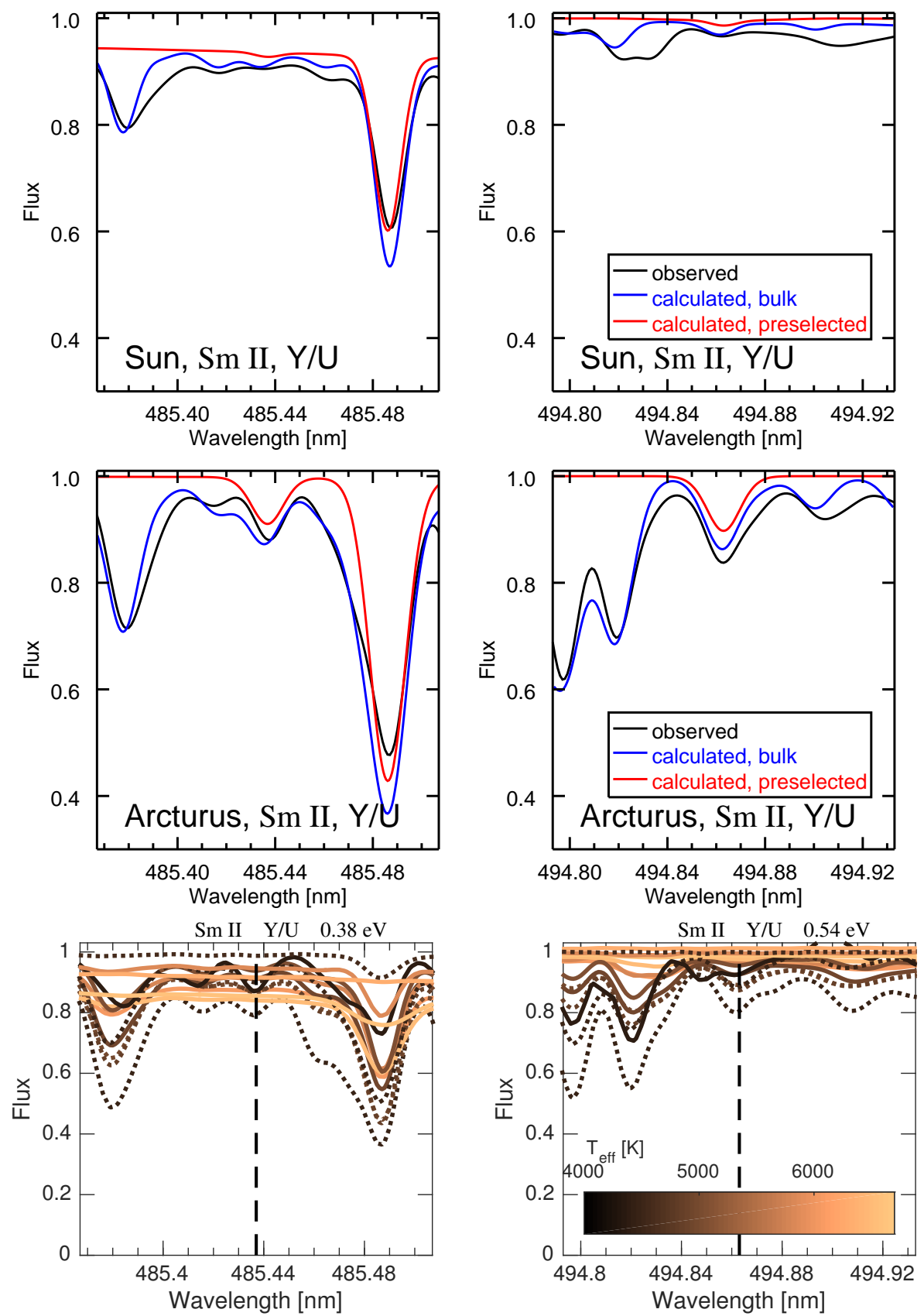

Fig. B.25. Observed and calculated line profiles around two preselected SmII lines for the Sun (top row) and Arcturus (middle row). Black lines: observations, red lines: calculations including preselected spectral lines only, blue lines: calculations including blends from background line list. Bottom row: line profiles generated from observed spectra of selected Gaia FGK benchmark stars. See Sect. 2.3.

should include the parameters of an r-process-rich, metal-poor star.

Gadolinium and Dysprosium have several stable isotopes, of which five and four contribute rather evenly to the Solar System Gd and Dy abundances, respectively (Table 3). Both elements have two isotopes with non-zero nuclear spin, giving rise to HFS. Den Hartog et al. (2006) observed partially resolved isotopic and hyperfine structure in the profiles for a few of the Gd II lines in their highest resolution FTS data, and Wickliffe et al. (2000) noticed partially resolved structure for many of the Dy II lines in their FTS spectra. However, most of the lines of both species were considered sufficiently narrow to be treated as single component lines in stellar abundance work (Lawler et al. 2009). 

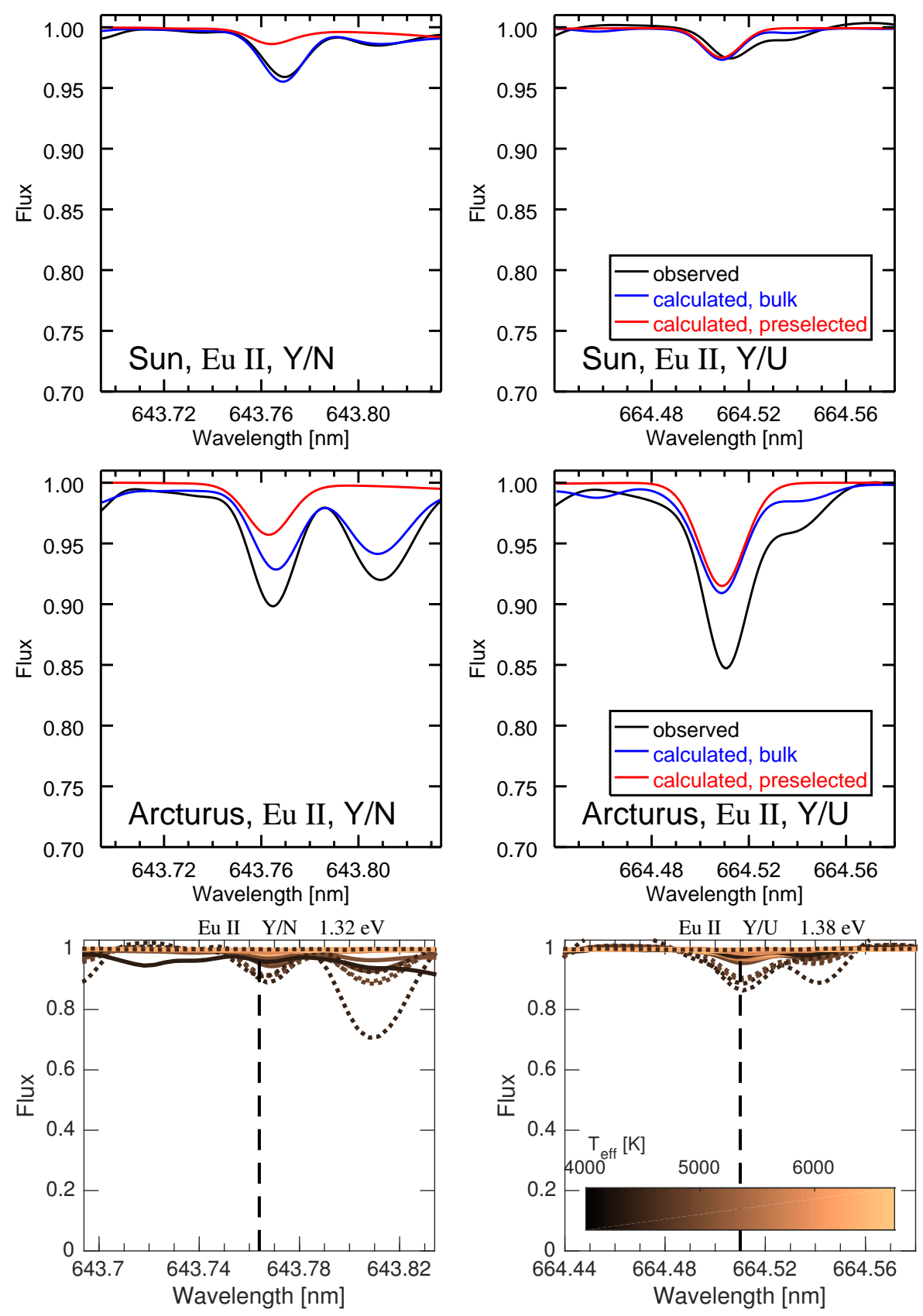

Fig. B.26. Observed and calculated line profiles around two preselected Eu II lines for the Sun (top row) and Arcturus (middle row). Black lines: observations, red lines: calculations including preselected spectral lines only, blue lines: calculations including blends from background line list. Bottom row: line profiles generated from observed spectra of selected Gaia FGK benchmark stars. See Sect. 2.3. 

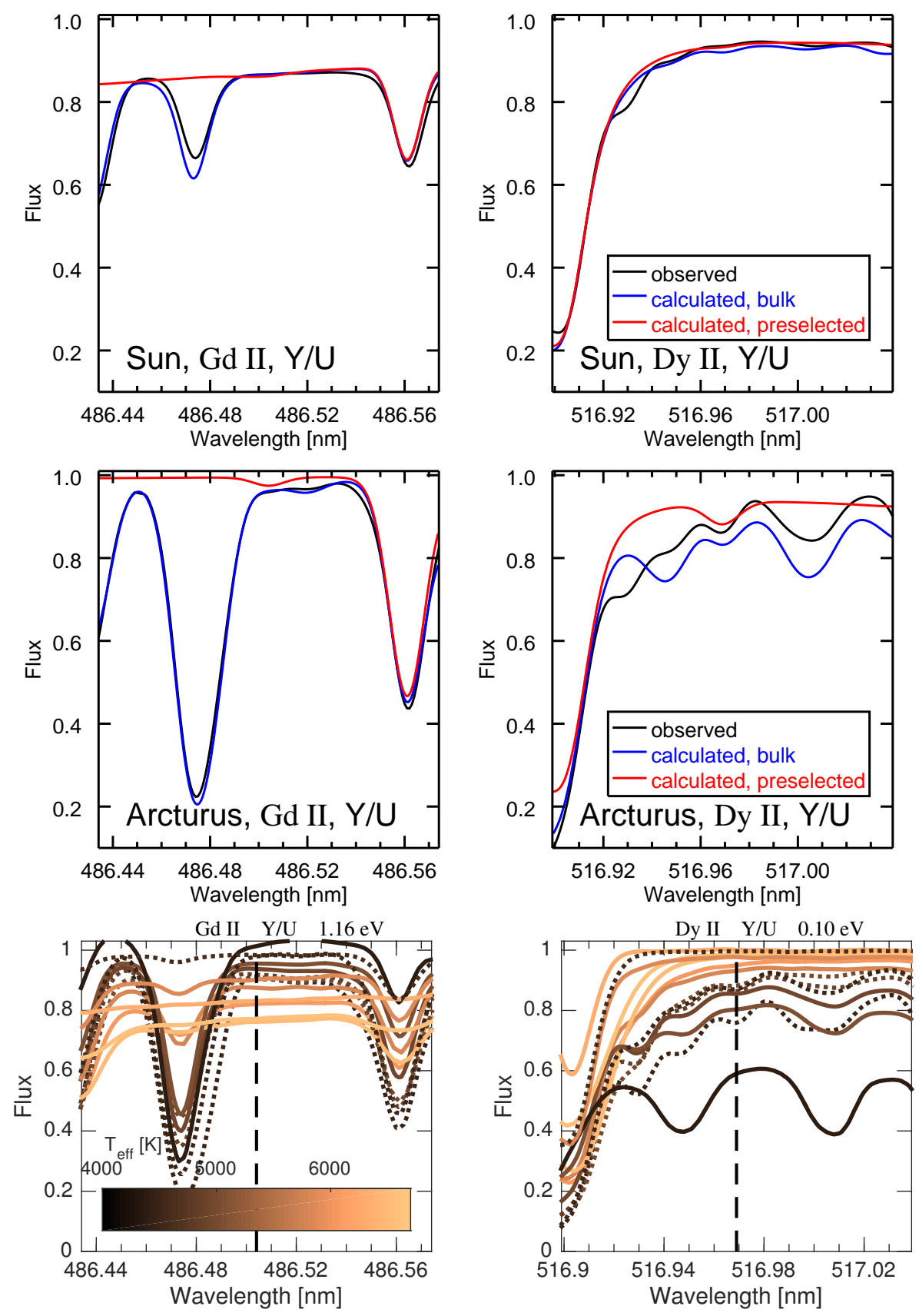

Fig. B.27. Observed and calculated line profiles around the preselected Gd II and Dy II lines for the Sun (top row) and Arcturus (middle row). Black lines: observations, red lines: calculations including preselected spectral lines only, blue lines: calculations including blends from background line list. Bottom row: line profiles generated from observed spectra of selected Gaia FGK benchmark stars. See Sect. 2.3. 


\section{Appendix C: Hyperfine structure and isotopic splitting data}

This appendix provides the HFS and IS data presented in Appendix B for several elements in the form of tables. If not stated otherwise, each table lists level energies $E$ in eV, HFS constants $A$ and $B$ in units of $\mathrm{cm}^{-1}$, representing magnetic dipole and electric quadrupole interactions for each level, as well as level designations and references. If applicable, isotopic wavelength shifts are given, in units of $\mathrm{cm}^{-1}$. Subscripts indicate baryon numbers for different isotopes.

Table C.1. HFS constants for 12 Sc I levels.

\begin{tabular}{ccccl}
\hline \hline$E(\mathrm{eV})$ & $A$ & $B$ & Level & Reference \\
\hline 0.000 & 0.0090 & -0.0009 & $4 \mathrm{~s}^{2}{ }^{2} \mathrm{D}$ & Childs (1971) \\
1.440 & 0.0083 & -0.0003 & $4 \mathrm{~s}^{4} \mathrm{~F}$ & Zeiske et al. (1976) \\
1.448 & 0.0095 & -0.0005 & $4 \mathrm{~s}{ }^{4} \mathrm{~F}$ & Ertmer \& Hofer (1976) \\
1.851 & 0.0100 & 0.0000 & $4 \mathrm{~s}^{2} \mathrm{~F}$ & Başar et al. (2004) \\
1.865 & -0.0008 & 0.0000 & $4 \mathrm{~s}^{2} \mathrm{~F}$ & Başar et al. (2004) \\
1.996 & -0.0115 & 0.0000 & $4 \mathrm{p}^{2} \mathrm{D}$ & Başar et al. (2004) \\
3.619 & 0.0029 & 0.0000 & $4 \mathrm{p}^{4} \mathrm{G}$ & Başar et al. (2004) \\
3.633 & 0.0015 & 0.0000 & $4 \mathrm{p}^{4} \mathrm{G}$ & Başar et al. (2004) \\
3.878 & 0.0019 & 0.0000 & $4 \mathrm{p}{ }^{4} \mathrm{~F}$ & Öztürk et al. (2007) \\
4.110 & 0.0034 & 0.0000 & $4 \mathrm{p}{ }^{2} \mathrm{G}$ & Öztürk et al. (2007) \\
4.111 & 0.0071 & 0.0000 & $4 \mathrm{p}{ }^{2} \mathrm{~F}$ & Öztürk et al. (2007) \\
4.179 & 0.0015 & 0.0000 & $4 \mathrm{p}{ }^{2} \mathrm{D}$ & Başar et al. (2004) \\
\hline
\end{tabular}

Table C.2. HFS constants for 53 V I levels.

\begin{tabular}{|c|c|c|c|c|}
\hline$(\mathrm{eV})$ & $A$ & $B$ & Level & Reference \\
\hline 40 & & & & Childs et al. (1979) \\
\hline & & & & Chil \\
\hline & & & $a^{6} \mathrm{D}$ & \\
\hline & 0.0135 & -0.0003 & $a^{6} \mathrm{D}$ & $\mathrm{Coc}$ \\
\hline & & & $a^{6} \mathrm{D}$ & \\
\hline & & & $a^{6} \mathrm{D}$ & \\
\hline 301 & 0.0 & 0.0 & $a^{6} \mathrm{D}$ & $\mathrm{Co}$ \\
\hline & & & $a^{4} D$ & \\
\hline & 2 & & $a^{4} D$ & \\
\hline & & & & \\
\hline 1.081 & -0.0053 & & $a^{4} D$ & $\mathrm{Ch}$ \\
\hline 1.183 & -0.0118 & 0.0 & $\mathrm{a}^{4} \mathrm{P}$ & \\
\hline & & & & \\
\hline 1218 & 0. & -0 . & $\mathrm{a}^{4} \mathrm{P}$ & \\
\hline & & & & \\
\hline 1.849 & 0.0011 & & $a^{4} \mathrm{H}$ & \\
\hline 1.853 & 0.0087 & -0.0004 & $\mathrm{a}^{4} \mathrm{H}$ & \\
\hline & & -0 . & $a^{4} \mathrm{H}$ & \\
\hline 2.242 & 0.0 & 0.0000 & $z^{6} \mathrm{D}$ & $\mathrm{Coc}$ \\
\hline .247 & 0.0 & -0.0001 & $z^{6} \mathrm{D}$ & $\mathrm{Co}$ \\
\hline .247 & -0.0117 & 0.0000 & $\mathrm{z}^{6} \mathrm{~F}$ & (1998) \\
\hline & & & & \\
\hline & & -0.0001 & $\mathrm{z}^{6} \mathrm{~F}$ & 1998) \\
\hline .264 & 0.0130 & 0.0000 & $\mathrm{z}^{6} \mathrm{~F}$ & 1998) \\
\hline 269 & & 0.0000 & $z^{6} \mathrm{D}$ & Cochrane et al. (1998) \\
\hline & & & $\mathrm{z}^{6} \mathrm{~F}$ & Cochrane et al. (1998) \\
\hline 2.286 & & 0.0001 & $z^{6} \mathrm{D}$ & Cochrane et al. (1998) \\
\hline 2.295 & 0.0156 & 0.0004 & $\mathrm{z}^{6} \mathrm{~F}$ & Cochrane et al. (1998) \\
\hline
\end{tabular}

Table C.2. continued.

\begin{tabular}{lcccl}
\hline \hline$E(\mathrm{eV})$ & $A$ & $B$ & Level & Reference \\
\hline 2.316 & 0.0165 & 0.0006 & $\mathrm{z}{ }^{6} \mathrm{~F}$ & Cochrane et al. (1998) \\
2.359 & 0.0133 & -0.0001 & $\mathrm{~b}^{2} \mathrm{H}$ & El-Kashef \& Ludwig (1992) \\
2.374 & 0.0054 & -0.0004 & $\mathrm{~b}{ }^{2} \mathrm{H}$ & El-Kashef \& Ludwig (1992) \\
2.582 & 0.0182 & 0.0000 & $\mathrm{z}{ }^{4} \mathrm{D}$ & Palmeri et al. (1995) \\
2.608 & 0.0204 & 0.0000 & $\mathrm{z}{ }^{4} \mathrm{D}$ & Palmeri et al. (1995) \\
3.071 & 0.0000 & 0.0000 & $\mathrm{z}{ }^{4} \mathrm{P}$ & none \\
3.089 & 0.0000 & 0.0000 & $\mathrm{z}{ }^{4} \mathrm{P}$ & none \\
3.116 & 0.0000 & 0.0000 & $\mathrm{z}{ }^{4} \mathrm{P}$ & none \\
3.131 & 0.0000 & 0.0000 & $\mathrm{y}{ }^{6} \mathrm{~F}$ & none \\
3.215 & 0.0212 & 0.0000 & $\mathrm{y}{ }^{4} \mathrm{~F}$ & Palmeri et al. (1995) \\
3.224 & 0.0072 & 0.0000 & $\mathrm{y}{ }^{4} \mathrm{~F}$ & Lefèbvre et al. (2002) \\
3.239 & 0.0058 & 0.0000 & $\mathrm{y}{ }^{4} \mathrm{~F}$ & Palmeri et al. (1995) \\
3.245 & 0.0028 & 0.0011 & $\mathrm{y}{ }^{4} \mathrm{~F}$ & Unkel et al. (1989) \\
3.246 & 0.0012 & 0.0000 & $\mathrm{y}{ }^{4} \mathrm{D}$ & Palmeri et al. (1995) \\
3.255 & 0.0002 & 0.0000 & $\mathrm{y}{ }^{4} \mathrm{D}$ & Palmeri et al. (1995) \\
3.266 & 0.0001 & 0.0000 & $\mathrm{z}{ }^{2} \mathrm{G}$ & Palmeri et al. (1995) \\
3.267 & 0.0000 & 0.0000 & $\mathrm{y}{ }^{4} \mathrm{D}$ & Palmeri et al. (1995) \\
3.283 & 0.0000 & 0.0000 & $\mathrm{y}{ }^{4} \mathrm{D}$ & Palmeri et al. (1995) \\
3.798 & 0.0002 & -0.0002 & $\mathrm{y}{ }^{4} \mathrm{G}$ & Unkel et al. (1989) \\
3.806 & 0.0106 & -0.0011 & $\mathrm{y}{ }^{4} \mathrm{G}$ & Unkel et al. (1989) \\
3.827 & 0.0186 & -0.0002 & $\mathrm{y}{ }^{4} \mathrm{G}$ & Unkel et al. (1989) \\
3.963 & 0.0000 & 0.0000 & $\mathrm{y}{ }^{2} \mathrm{~S}$ & Lefèbvre et al. (2002) \\
4.609 & 0.0000 & 0.0000 & $\mathrm{v}{ }^{2} \mathrm{G}$ & none \\
4.739 & 0.0000 & 0.0000 & $\mathrm{x}{ }^{2} \mathrm{H}$ & none \\
5.251 & 0.0000 & 0.0000 & $\mathrm{e}{ }^{6} \mathrm{G}$ & none \\
\hline
\end{tabular}


Table C.3. HFS constants for $35 \mathrm{Mn}$ I levels.

\begin{tabular}{|c|c|c|c|c|}
\hline$E(\mathrm{eV})$ & $A$ & $B$ & Level & Reference \\
\hline 0.000 & -0.0024 & 0.0 & $a^{6} \mathrm{~S}$ & Davis et al. (1971) \\
\hline 2.143 & 0.0153 & 0.0007 & $a^{6} \mathrm{D}$ & Dembczyński et al. (1979) \\
\hline 2.164 & 0.0146 & -0.0015 & $a^{6} \mathrm{D}$ & Dembczyński et al. (1979) \\
\hline 2.178 & 0.0157 & -0.0022 & $a^{6} \mathrm{D}$ & Dembczyński et al. (1979) \\
\hline 2.187 & 0.0294 & 0.0 & $a^{6} \mathrm{D}$ & Dembczyński et al. (1979) \\
\hline 2.282 & 0.0191 & 0.0009 & $\mathrm{z}^{8} \mathrm{P}$ & Brodzinski et al. (1987) \\
\hline 2.298 & 0.0182 & -0.0034 & $\mathrm{z}^{8} \mathrm{P}$ & Brodzinski et al. (1987) \\
\hline 2.319 & 0.0152 & 0.0016 & $\mathrm{z}^{8} \mathrm{P}$ & Brodzinski et al. (1987) \\
\hline 2.888 & -0.0054 & 0.0 & $a^{4} D$ & Blackwell-Whitehead et al. (2005b) \\
\hline 2.920 & -0.0046 & 0.0 & $a^{4} D$ & Blackwell-Whitehead et al. (2005b) \\
\hline 2.941 & 0.0017 & 0.0 & $a^{4} D$ & Blackwell-Whitehead et al. (2005b) \\
\hline 2.953 & 0.0506 & 0.0 & $a^{4} D$ & Blackwell-Whitehead et al. (2005b) \\
\hline 3.072 & 0.0191 & 0.0004 & $\mathrm{z}^{6} \mathrm{P}$ & Handrich et al. (1969) \\
\hline 3.073 & 0.0156 & -0.0025 & $\mathrm{z}^{6} \mathrm{P}$ & Handrich et al. (1969) \\
\hline 3.075 & 0.0143 & 0.0021 & $\mathrm{z}^{6} \mathrm{P}$ & Handrich et al. (1969) \\
\hline 3.133 & 0.0135 & 0.0 & $a^{4} G$ & Johann et al. (1981) \\
\hline 3.134 & 0.0199 & 0.0 & $a^{4} G$ & Johann et al. (1981) \\
\hline 3.772 & 0.0096 & 0.0 & $b^{4} \mathrm{D}$ & Blackwell-Whitehead et al. (2005b) \\
\hline 3.844 & -0.0203 & 0.0025 & $\mathrm{z}^{4} \mathrm{P}$ & Brodzinski et al. (1987) \\
\hline 3.853 & -0.0271 & -0.0013 & $\mathrm{z}^{4} \mathrm{P}$ & Brodzinski et al. (1987) \\
\hline 4.425 & -0.0324 & 0.0006 & $\mathrm{y}^{6} \mathrm{P}$ & Luc \& Gerstenkorn (1972) \\
\hline 4.429 & -0.018 & -0.0023 & $\mathrm{y}^{6} \mathrm{P}$ & Luc \& Gerstenkorn (1972) \\
\hline 4.435 & -0.013 & -0.0062 & $\mathrm{y}^{6} \mathrm{P}$ & Luc \& Gerstenkorn (1972) \\
\hline 4.889 & 0.0246 & 0.0016 & $e^{8} \mathrm{~S}$ & Brodzinski et al. (1987) \\
\hline 5.133 & 0.027 & 0.0 & $e^{6} S$ & Brodzinski et al. (1987) \\
\hline 5.396 & 0.0046 & 0.0 & $\mathrm{z}^{6} \mathrm{~F}$ & Lefèbvre et al. (2003) \\
\hline 5.491 & 0.0044 & 0.0 & $z^{4} F$ & Blackwell-Whitehead et al. (2005b) \\
\hline 5.520 & 0.0057 & 0.0 & $z^{4} F$ & Blackwell-Whitehead et al. (2005b) \\
\hline 5.542 & 0.0095 & 0.0 & $z^{4} F$ & Blackwell-Whitehead et al. (2005b) \\
\hline 5.556 & 0.0223 & 0.0 & $z^{4} F$ & Blackwell-Whitehead et al. (2005b) \\
\hline 5.696 & 0.0027 & 0.0 & $\mathrm{z}^{4} \mathrm{D}$ & Başar et al. (2003) \\
\hline 5.853 & 0.0155 & 0.0 & $e^{6} D$ & Luc \& Gerstenkorn (1972) \\
\hline 5.854 & 0.0158 & 0.0 & $e^{6} D$ & Luc \& Gerstenkorn (1972) \\
\hline 5.854 & 0.0176 & 0.0 & $e^{6} \mathrm{D}$ & Luc \& Gerstenkorn (1972) \\
\hline 6.149 & -0.0505 & 0.0 & $e^{4} S$ & Lefèbvre et al. (2003) \\
\hline
\end{tabular}


Table C.4. HFS constants for 47 Co I levels measured by Pickering (1996).

\begin{tabular}{|c|c|c|c|}
\hline$E(\mathrm{eV})$ & $A$ & $B$ & Level \\
\hline .710 & 0.0059 & -0.008 & $a^{4} P$ \\
\hline 1.740 & 0.0106 & 0.004 & $a^{4} P$ \\
\hline .785 & -0.0236 & 0.0 & $a^{4} P$ \\
\hline 1.883 & 0.0374 & 0.005 & $b^{4} \mathrm{P}$ \\
\hline 1.956 & 0.0154 & -0.002 & $b^{4} \mathrm{P}$ \\
\hline .042 & 0.0205 & 0.002 & $a^{2} G$ \\
\hline 2.080 & 0.0463 & 0.004 & $a^{2} D$ \\
\hline .137 & 0.028 & -0.0032 & $a^{2} G$ \\
\hline 2.280 & 0.0112 & 0.004 & $\mathrm{a}^{2} \mathrm{P}$ \\
\hline 2.328 & 0.0201 & 0.0 & $\mathrm{a}^{2} \mathrm{P}$ \\
\hline 3.117 & 0.0253 & 0.008 & $z^{6} G$ \\
\hline 3.216 & 0.0206 & 0.005 & $z^{6} G$ \\
\hline 3.252 & 0.0183 & 0.006 & $z^{6} G$ \\
\hline 3.298 & 0.0049 & 0.004 & $z^{6} G$ \\
\hline 3.514 & 0.0270 & -0.002 & $z^{4} F$ \\
\hline 3.576 & 0.0258 & 0.007 & $z^{4} G$ \\
\hline 3.629 & 0.0173 & 0.006 & $z^{4} G$ \\
\hline 3.632 & 0.0251 & 0.00 & $z^{4} D$ \\
\hline 3.713 & 0.0232 & 0.001 & $z^{4} \mathrm{D}$ \\
\hline 3.775 & 0.0235 & 0.001 & $z^{4} D$ \\
\hline 3.812 & 0.0275 & 0.0 & $z^{4} D$ \\
\hline 3.952 & 0.015 & 0.006 & $z^{2} F$ \\
\hline 3.971 & 0.016 & 0.005 & $\mathrm{y}^{4} \mathrm{D}$ \\
\hline
\end{tabular}

Table C.4. continued

\begin{tabular}{cccc}
\hline \hline$E(\mathrm{eV})$ & $A$ & $B$ & Level \\
\hline 4.021 & 0.0100 & 0.0 & $\mathrm{y}{ }^{4} \mathrm{G}$ \\
4.025 & 0.0111 & 0.0 & $\mathrm{y}{ }^{4} \mathrm{G}$ \\
4.049 & 0.0155 & 0.00 & $\mathrm{y}{ }^{4} \mathrm{D}$ \\
4.064 & 0.0349 & 0.0 & $\mathrm{z}{ }^{2} \mathrm{~F}$ \\
4.110 & 0.0197 & 0.00 & $\mathrm{y}^{4} \mathrm{D}$ \\
4.149 & 0.0154 & 0.00 & $\mathrm{z}^{2} \mathrm{D}$ \\
4.149 & 0.0129 & 0.0 & $\mathrm{y}^{4} \mathrm{G}$ \\
4.232 & 0.0248 & -0.004 & $\mathrm{y}^{2} \mathrm{G}$ \\
4.240 & 0.0397 & -0.002 & $\mathrm{y}^{4} \mathrm{~F}$ \\
4.259 & 0.0461 & 0.002 & $\mathrm{z}^{2} \mathrm{D}$ \\
4.475 & 0.0164 & 0.00 & $\mathrm{y}^{2} \mathrm{D}$ \\
4.572 & 0.042 & 0.00 & $\mathrm{y}^{2} \mathrm{D}$ \\
5.552 & 0.0134 & 0.00 & $\mathrm{e}^{4} \mathrm{~F}$ \\
5.663 & 0.0335 & 0.005 & $\mathrm{e}^{6} \mathrm{~F}$ \\
5.791 & 0.0287 & -0.001 & $\mathrm{e}^{6} \mathrm{~F}$ \\
5.839 & 0.0257 & -0.001 & $\mathrm{e}^{6} \mathrm{~F}$ \\
5.873 & 0.0194 & -0.001 & $\mathrm{e}^{6} \mathrm{~F}$ \\
5.892 & 0.0360 & 0.005 & $\mathrm{f}^{4} \mathrm{~F}$ \\
5.976 & 0.0283 & 0.003 & $\mathrm{f}^{4} \mathrm{~F}$ \\
6.341 & 0.0000 & 0.0 & $\mathrm{e}^{4} \mathrm{H}$ \\
6.345 & 0.0000 & 0.00 & $\mathrm{e}^{4} \mathrm{H}$ \\
6.348 & 0.0000 & 0.00 & $\mathrm{e}^{4} \mathrm{G}$ \\
6.462 & 0.0000 & 0.00 & $\mathrm{e}^{4} \mathrm{H}$ \\
6.543 & 0.0000 & 0.00 & $\mathrm{e}^{4} \mathrm{G}$ \\
\hline & & &
\end{tabular}

Table C.5. HFS constants and isotopic shifts relative to the ground state $\left(4 \mathrm{~s}^{2} \mathrm{~S}\right)$ for five $\mathrm{Cu}$ I levels.

\begin{tabular}{ccccccccll}
\hline \hline$E(\mathrm{eV})$ & $A_{63}$ & $B_{63}$ & $\mathrm{IS}_{63}$ & $A_{65}$ & $B_{65}$ & $\mathrm{IS}_{65}$ & Level & Ref. (HFS) & Ref. (IS) $^{(a)}$ \\
\hline 1.389 & 0.0250 & 0.0062 & 0.0168 & 0.0268 & 0.0058 & -0.0377 & $4 \mathrm{~s}^{2}{ }^{2} \mathrm{D}$ & Fischer et al. (1967) & Kurucz (2012), Wagner (1955) \\
1.642 & 0.0619 & 0.0046 & 0.0170 & 0.0661 & 0.0043 & -0.0381 & $4 \mathrm{~s}^{2}{ }^{2} \mathrm{D}$ & Bergström et al. (1989) & Kurucz (2012), Wagner (1955) \\
3.786 & 0.0169 & 0.0 & -0.0059 & 0.0181 & 0.0 & 0.0132 & $4 \mathrm{p}{ }^{2} \mathrm{P}$ & Hermann et al. (1993) & Kurucz (2012), Wagner (1955) \\
3.817 & 0.0065 & -0.0010 & -0.0062 & 0.0069 & -0.0009 & 0.0139 & $4 \mathrm{p}{ }^{2} \mathrm{P}$ & Hermann et al. (1993) & Kurucz (2012), Wagner (1955) \\
6.191 & 0.0 & 0.0 & -0.006 & 0.0 & 0.0 & 0.013 & $4 \mathrm{~d}^{2} \mathrm{D}$ & none & Kurucz (2012) \\
\hline
\end{tabular}

Notes. ${ }^{(a)}$ http://kurucz . harvard.edu/atoms/2900/ab290063. dat, http://kurucz . harvard. edu/atoms/2900/ab290065.dat

Table C.6. HFS constants for five Ba II levels, for isotopes with non-zero nuclear spin.

\begin{tabular}{lcccccl}
\hline \hline$E(\mathrm{eV})$ & $A_{135}$ & $B_{135}$ & $A_{137}$ & $B_{137}$ & Level & Reference \\
\hline 0.000 & 0.1198 & 0.0 & 0.1341 & 0.0 & $6 \mathrm{~s}^{2} \mathrm{~S}$ & Becker et al. (1981) \\
0.604 & 0.0057 & 0.0010 & 0.0063 & 0.0015 & $5 \mathrm{~d}^{2} \mathrm{D}$ & Silverans et al. (1986) \\
0.704 & -0.0004 & 0.0013 & -0.0004 & 0.0020 & $5 \mathrm{~d}^{2} \mathrm{D}$ & Silverans et al. (1986) \\
2.512 & 0.0222 & 0.0 & 0.0248 & 0.0 & $6 \mathrm{p}^{2} \mathrm{P}$ & Villemoes et al. (1993) \\
2.722 & 0.0038 & 0.002 & 0.0042 & 0.0031 & $6 \mathrm{p}{ }^{2} \mathrm{P}$ & Villemoes et al. (1993) \\
\hline
\end{tabular}


Table C.7. HFS constants for nine La II levels.

\begin{tabular}{ccccl}
\hline \hline$E(\mathrm{eV})$ & $A$ & $B$ & Level & Reference \\
\hline 0.173 & $3.165 \times 10^{-2}$ & $1.66 \times 10^{-3}$ & $6 \mathrm{~s} \mathrm{a}^{1} \mathrm{D}$ & Höhle et al. (1982) \\
0.235 & $-3.760 \times 10^{-2}$ & $1.66 \times 10^{-3}$ & $6 \mathrm{~s} \mathrm{a}^{3} \mathrm{D}$ & Höhle et al. (1982) \\
0.244 & $-6.19 \times 10^{-4}$ & $1.25 \times 10^{-3}$ & $5 \mathrm{~d}^{2} \mathrm{a}^{3} \mathrm{~F}$ & Höhle et al. (1982) \\
0.321 & $-3 \times 10^{-4}$ & $1.89 \times 10^{-3}$ & $6 \mathrm{~s} \mathrm{a}^{3} \mathrm{D}$ & Höhle et al. (1982) \\
2.261 & $4.96 \times 10^{-3}$ & $1.5 \times 10^{-4}$ & $5 \mathrm{~d} \mathrm{y}^{3} \mathrm{~F}^{\mathrm{o}}$ & Höhle et al. (1982) \\
2.658 & $1.44 \times 10^{-2}$ & - & $5 \mathrm{~d} \mathrm{z}^{3} \mathrm{D}^{\mathrm{o}}$ & Lawler et al. (2001a) \\
2.741 & $4.26 \times 10^{-3}$ & $9 \times 10^{-5}$ & $5 \mathrm{~d} \mathrm{z}^{3} \mathrm{D}^{\circ}$ & Li et al. (2001) \\
2.763 & $4.5 \times 10^{-3}$ & $2 \times 10^{-3}$ & $5 \mathrm{~d} \mathrm{z}^{3} \mathrm{G}^{\text {o }}$ & Furmann et al. (2008) \\
2.815 & $2.6 \times 10^{-3}$ & - & $5 \mathrm{~d} \mathrm{z}^{3} \mathrm{P}^{\mathrm{o}}$ & Lawler et al. (2001a) \\
\hline
\end{tabular}

Notes. ${ }^{(a)}$ Not included in the line list.

Table C.8. HFS constant $A$ for 12 Pr II levels.

\begin{tabular}{cccl}
\hline \hline$E(\mathrm{eV})$ & $A$ & Level & Reference \\
\hline 0.483 & $3.01 \times 10^{-2}$ & $5 \mathrm{~d}^{5} \mathrm{~L}^{\mathrm{o}}$ & Ginibre (1989) \\
0.508 & $3.25 \times 10^{-2}$ & $5 \mathrm{~d}^{5} \mathrm{~K}^{\mathrm{o}}$ & Ginibre (1989) \\
0.633 & $2.22 \times 10^{-2}$ & $5 \mathrm{~d}^{5} \mathrm{~L}^{\mathrm{o}}$ & Ginibre (1989) \\
0.648 & $2.40 \times 10^{-2}$ & $5 \mathrm{~d}^{5} \mathrm{~K}^{\mathrm{o}}$ & Ginibre (1989) \\
0.795 & $1.88 \times 10^{-2}$ & $5 \mathrm{~d}^{5} \mathrm{~K}^{\mathrm{o}}$ & Ginibre (1989) \\
0.796 & $1.82 \times 10^{-2}$ & $5 \mathrm{~d}^{5} \mathrm{~L}^{\mathrm{o}}$ & Ginibre (1989) \\
0.968 & $1.50 \times 10^{-2}$ & $5 \mathrm{~d}^{5} \mathrm{~L}^{\mathrm{o}}$ & Ginibre (1989) \\
2.811 & $2.554 \times 10^{-2}$ & $6 \mathrm{p} 5$ & Rivest et al. (2002) \\
2.884 & $1.949 \times 10^{-2}$ & $5 \mathrm{~d}^{2}$ & Rivest et al. (2002) \\
2.990 & $2.740 \times 10^{-2}$ & $6 \mathrm{p} 5 \mathrm{~K}$ & Rivest et al. (2002) \\
3.170 & $2.301 \times 10^{-2}$ & $6 \mathrm{p}{ }^{5} \mathrm{~K}$ & Rivest et al. (2002) \\
3.363 & $1.973 \times 10^{-2}$ & $6 \mathrm{p}{ }^{5} \mathrm{~K}$ & Rivest et al. (2002) \\
\hline
\end{tabular}

Table C.9. Wavelength shifts for isotopes with baryon numbers 150 and 142 relative to isotope 144 for the 53 preselected Nd II lines.

\begin{tabular}{|c|c|c|c|c|c|c|}
\hline $\begin{array}{l}\lambda^{(a)} \\
(\AA)\end{array}$ & $\begin{array}{l}E_{\text {low }} \\
(\mathrm{eV})\end{array}$ & $\begin{array}{l}E_{\text {upp }} \\
(\mathrm{eV})\end{array}$ & $\operatorname{HFS}^{(b)}$ & $\begin{array}{c}\mathrm{IS}(144-150) \\
(\AA)\end{array}$ & Ref.(IS) & $\begin{array}{c}\mathrm{IS}(142-144)^{(c)} \\
(\AA)\end{array}$ \\
\hline 4811.342 & 0.064 & 2.640 & $E_{\text {low }}$ & 0.0088 & 11 & 0.0025 \\
\hline 4849.062 & 0.471 & 3.028 & both & 0.0052 & 11 & 0.0012 \\
\hline 4859.03 & 0.321 & 2.871 & both & -0.0040 & 11 & -0.0012 \\
\hline 4902.04 & 0.064 & 2.592 & $E_{\text {low }}$ & 0.0202 & 11 & 0.0063 \\
\hline 4914.38 & 0.380 & 2.902 & both & 0.0138 & 11 & 0.0041 \\
\hline 4943.899 & 0.205 & 2.712 & $E_{\text {low }}$ & -0.0007 & 11 & -0.0005 \\
\hline 4947.02 & 0.559 & 3.065 & both & 0.0198 & 11 & 0.0054 \\
\hline 4959.12 & 0.064 & 2.563 & $E_{\text {low }}$ & 0.0224 & 11 & 0.0066 \\
\hline 4961.387 & 0.631 & 3.129 & $E_{\text {low }}$ & -0.0005 & 12 & \\
\hline 4970.91 & 0.321 & 2.814 & both & 0.0237 & 11 & 0.0067 \\
\hline 4987.16 & 0.742 & 3.227 & both & 0.0134 & 11 & 0.0040 \\
\hline 4989.95 & 0.631 & 3.115 & $E_{\text {low }}$ & -0.0105 & 11 & -0.0032 \\
\hline 4998.541 & 0.471 & 2.951 & $E_{\text {low }}$ & -0.0065 & 11 & 0.0025 \\
\hline 5089.832 & 0.205 & 2.640 & $E_{\text {low }}$ & 0.0086 & 11 & 0.0023 \\
\hline
\end{tabular}

Notes. ${ }^{(a)}$ Average wavelengths. ${ }^{(b)}$ Indicates if HFS data are available for both levels, for $E_{\text {low }}$ or $E_{\text {upp }}$ only (see Table C.10), or for none. ${ }^{\left({ }^{(}\right)}$Level energy shifts between isotopes 142 and 144 were taken from Ahmad \& Saksena (1981).

References. References for level energy shifts between isotopes 144 and 150 used to calculate the wavelength shifts are given in column "Ref.(IS)": (1) Nakhate et al. (1997), (2) Blaise et al. (1984). The two numbers in each row refer to $E_{\text {low }}$ and $E_{\text {upp }}$, respectively. 
Table C.9. continued.

\begin{tabular}{|c|c|c|c|c|c|c|}
\hline $\begin{array}{l}\lambda^{(a)} \\
(\AA)\end{array}$ & $\begin{array}{l}E_{\text {low }} \\
(\mathrm{eV})\end{array}$ & $\begin{array}{l}E_{\text {upp }} \\
(\mathrm{eV})\end{array}$ & $\operatorname{HFS}^{(b)}$ & $\begin{array}{c}\text { IS }(144-150) \\
(\AA)\end{array}$ & Ref.(IS) & $\begin{array}{c}\mathrm{IS}(142-144)^{(c)} \\
(\AA)\end{array}$ \\
\hline 5092.79 & 0.380 & 2.814 & both & 0.0249 & 11 & 0.0065 \\
\hline 5130.59 & 1.304 & 3.720 & none & -0.0061 & 11 & -0.0024 \\
\hline 5132.33 & 0.559 & 2.975 & both & 0.0137 & 11 & 0.0037 \\
\hline 5143.34 & 0.182 & 2.592 & $E_{\text {low }}$ & 0.0225 & 11 & 0.0069 \\
\hline 5165.13 & 0.680 & 3.080 & $E_{\text {upp }}$ & -0.0667 & 11 & -0.0198 \\
\hline 5200.121 & 0.559 & 2.943 & $E_{\text {low }}$ & 0.0162 & 12 & \\
\hline 5212.36 & 0.205 & 2.583 & $E_{\text {low }}$ & 0.0139 & 11 & 0.0041 \\
\hline 5234.19 & 0.550 & 2.918 & both & -0.0411 & 11 & -0.0118 \\
\hline 5249.58 & 0.976 & 3.337 & $E_{\text {upp }}$ & -0.0174 & 21 & \\
\hline 5250.81 & 0.745 & 3.105 & none & -0.0532 & 11 & -0.0154 \\
\hline 5255.51 & 0.205 & 2.563 & $E_{\text {low }}$ & 0.0238 & 11 & 0.0069 \\
\hline 5276.869 & 0.859 & 3.208 & both & -0.0518 & 11 & -0.0153 \\
\hline 5293.16 & 0.823 & 3.165 & $E_{\text {upp }}$ & -0.0193 & 11 & -0.0056 \\
\hline 5306.46 & 0.859 & 3.195 & $E_{\text {low }}$ & -0.0462 & 11 & -0.0135 \\
\hline 5310.04 & 1.136 & 3.471 & $E_{\text {upp }}$ & -0.0671 & 11 & -0.0195 \\
\hline 5311.45 & 0.986 & 3.319 & $E_{\text {upp }}$ & -0.0395 & 11 & -0.0116 \\
\hline 5319.81 & 0.550 & 2.880 & both & -0.0394 & 11 & -0.0110 \\
\hline 5356.97 & 1.264 & 3.578 & $E_{\text {upp }}$ & -0.0413 & 11 & -0.0123 \\
\hline 5361.17 & 0.559 & 2.871 & both & -0.0049 & 11 & -0.0020 \\
\hline 5361.467 & 0.680 & 2.992 & $E_{\text {upp }}$ & -0.0411 & 11 & -0.0115 \\
\hline 5416.374 & 0.859 & 3.148 & both & -0.0681 & 11 & -0.0203 \\
\hline 5431.52 & 1.121 & 3.403 & $E_{\text {upp }}$ & -0.0469 & 11 & -0.0121 \\
\hline 5442.264 & 0.680 & 2.958 & $E_{\text {upp }}$ & -0.0587 & 11 & -0.0175 \\
\hline 5485.70 & 1.264 & 3.524 & $E_{\text {upp }}$ & -0.0238 & 11 & -0.0072 \\
\hline 5533.82 & 0.559 & 2.799 & $E_{\text {low }}$ & 0.0211 & 11 & 0.0061 \\
\hline 5548.45 & 0.550 & 2.784 & both & -0.0585 & 11 & -0.0182 \\
\hline 5581.59 & 0.859 & 3.080 & both & -0.0779 & 11 & -0.0231 \\
\hline 5618.99 & 1.773 & 3.979 & none & -0.0531 & 21 & \\
\hline 5740.86 & 1.160 & 3.319 & $E_{\text {upp }}$ & -0.0455 & 11 & -0.0135 \\
\hline 5811.57 & 0.859 & 2.992 & both & -0.0483 & 11 & -0.0135 \\
\hline 5842.366 & 1.282 & 3.403 & $E_{\text {upp }}$ & -0.0540 & 21 & \\
\hline 5882.786 & 0.559 & 2.666 & $E_{\text {low }}$ & 0.0284 & 12 & \\
\hline 6365.54 & 0.933 & 2.880 & $E_{\text {upp }}$ & -0.0564 & 11 & -0.0158 \\
\hline 6385.154 & 1.160 & 3.101 & none & -0.0779 & 11 & \\
\hline 6637.19 & 1.452 & 3.319 & $E_{\text {upp }}$ & -0.0621 & 21 & \\
\hline 8530.545 & 0.064 & 1.517 & $E_{\text {low }}$ & 0.0539 & 12 & \\
\hline 8594.883 & 1.140 & 2.583 & none & -0.1293 & 21 & \\
\hline 8643.48 & 1.200 & 2.634 & none & -0.2542 & 22 & \\
\hline 8691.303 & 1.350 & 2.776 & none & -0.1738 & 21 & \\
\hline
\end{tabular}

Table C.10. HFS constants for 34 out of the $69 \mathrm{Nd}$ II levels involved in the preselected transitions.

\begin{tabular}{lrcrcr}
\hline \hline$E(\mathrm{eV})$ & \multicolumn{1}{c}{$A_{143}$} & $B_{143}$ & \multicolumn{1}{c}{$A_{145}$} & $B_{145}$ & Level \\
\hline 0.064 & $-1.123 \times 10^{-2}$ & $4.39 \times 10^{-3}$ & $-6.979 \times 10^{-3}$ & $2.29 \times 10^{-3}$ & $6 \mathrm{~s}{ }^{6} \mathrm{I}$ \\
0.182 & $1.028 \times 10^{-2}$ & $3.57 \times 10^{-3}$ & $6.390 \times 10^{-3}$ & $1.86 \times 10^{-3}$ & $6 \mathrm{~s}{ }^{6} \mathrm{I}$ \\
0.205 & $-1.981 \times 10^{-3}$ & $4.24 \times 10^{-3}$ & $-1.231 \times 10^{-3}$ & $2.21 \times 10^{-3}$ & $6 \mathrm{~s}{ }^{4} \mathrm{I}$ \\
0.321 & $-9.405 \times 10^{-3}$ & $1.00 \times 10^{-5}$ & $-5.846 \times 10^{-3}$ & $5.21 \times 10^{-6}$ & $6 \mathrm{~s}{ }^{6} \mathrm{I}$ \\
0.380 & $-2.578 \times 10^{-4}$ & $3.08 \times 10^{-3}$ & $-1.603 \times 10^{-4}$ & $1.61 \times 10^{-3}$ & $6 \mathrm{~s}{ }^{4} \mathrm{I}$ \\
0.471 & $-8.608 \times 10^{-3}$ & $7.27 \times 10^{-3}$ & $-5.350 \times 10^{-3}$ & $3.79 \times 10^{-3}$ & ${ }^{6 \mathrm{~s}}{ }^{6} \mathrm{I}$ \\
0.550 & $-4.736 \times 10^{-3}$ & $9.34 \times 10^{-4}$ & $-2.943 \times 10^{-3}$ & $4.87 \times 10^{-4}$ & $5 \mathrm{~d}{ }^{6} \mathrm{~L}$ \\
0.559 & $3.936 \times 10^{-4}$ & $1.35 \times 10^{-3}$ & $2.447 \times 10^{-4}$ & $7.04 \times 10^{-4}$ & $6 \mathrm{~s}{ }^{4} \mathrm{I}$ \\
0.631 & $-8.096 \times 10^{-3}$ & $6.50 \times 10^{-3}$ & $-5.032 \times 10^{-3}$ & $3.39 \times 10^{-3}$ & $6 \mathrm{~s}{ }^{6} \mathrm{I}$ \\
\hline
\end{tabular}

Notes. The data are from Rosner et al. (2005), except as noted for two levels. The subscripts indicate baryon numbers for isotopes ${ }^{143} \mathrm{Nd}$ and ${ }^{145} \mathrm{Nd}$ with nuclear spin $I=3.5$. ${ }^{(a)}$ Data from Ma \& Yang (2004). 
Table C.10. continued.

\begin{tabular}{|c|c|c|c|c|c|}
\hline$E(\mathrm{eV})$ & $A_{143}$ & $B_{143}$ & $A_{145}$ & $B_{145}$ & Level \\
\hline 0.742 & $5.551 \times 10^{-4}$ & $3.47 \times 10^{-3}$ & $3.450 \times 10^{-4}$ & $1.81 \times 10^{-3}$ & $6 s{ }^{4} I$ \\
\hline $0.745^{(a)}$ & $-6.671 \times 10^{-3}$ & $-3.88 \times 10^{-3}$ & $-4.140 \times 10^{-3}$ & $2.23 \times 10^{-3}$ & $5 \mathrm{~d}^{6} \mathrm{~K}$ \\
\hline $0.859^{(a)}$ & $-5.030 \times 10^{-3}$ & $1.82 \times 10^{-3}$ & $-3.119 \times 10^{-3}$ & $9.37 \times 10^{-4}$ & $5 \mathrm{~d}^{6} \mathrm{~K}$ \\
\hline 2.784 & $-6.301 \times 10^{-3}$ & $-2.45 \times 10^{-3}$ & $-3.917 \times 10^{-3}$ & $-1.28 \times 10^{-3}$ & o \\
\hline 2.814 & $-5.092 \times 10^{-3}$ & $5.57 \times 10^{-3}$ & $-3.165 \times 10^{-3}$ & $2.90 \times 10^{-3}$ & $5 d^{2}{ }^{4} I^{o}$ \\
\hline 2.871 & $-6.492 \times 10^{-3}$ & $-3.80 \times 10^{-3}$ & $-4.036 \times 10^{-3}$ & $-1.98 \times 10^{-3}$ & 0 \\
\hline 2.880 & $-6.639 \times 10^{-3}$ & $-3.38 \times 10^{-3}$ & $-4.127 \times 10^{-3}$ & $-1.76 \times 10^{-3}$ & $6 \mathrm{p}^{6} \mathrm{~K}^{\mathrm{o}}$ \\
\hline 2.902 & $-4.765 \times 10^{-3}$ & $4.80 \times 10^{-4}$ & $-2.962 \times 10^{-3}$ & $2.50 \times 10^{-4}$ & o \\
\hline 2.918 & $-6.016 \times 10^{-3}$ & $-1.60 \times 10^{-3}$ & $-3.740 \times 10^{-3}$ & $-8.34 \times 10^{-4}$ & o \\
\hline 2.958 & $-3.959 \times 10^{-3}$ & $-8.21 \times 10^{-4}$ & $-2.461 \times 10^{-3}$ & $-4.28 \times 10^{-4}$ & o \\
\hline 2.975 & $-4.892 \times 10^{-3}$ & $6.00 \times 10^{-3}$ & $-3.041 \times 10^{-3}$ & $3.13 \times 10^{-3}$ & o \\
\hline 2.992 & $-4.930 \times 10^{-3}$ & $3.94 \times 10^{-4}$ & $-3.064 \times 10^{-3}$ & $2.05 \times 10^{-4}$ & o \\
\hline 3.028 & $-4.817 \times 10^{-3}$ & $-1.83 \times 10^{-3}$ & $-2.994 \times 10^{-3}$ & $-9.56 \times 10^{-4}$ & 0 \\
\hline 3.065 & $-4.463 \times 10^{-3}$ & $-2.57 \times 10^{-3}$ & $-2.774 \times 10^{-3}$ & $-1.34 \times 10^{-3}$ & ${ }^{4} \mathrm{I}^{\mathrm{o}}$ \\
\hline 3.080 & $-3.629 \times 10^{-3}$ & $-1.00 \times 10^{-4}$ & $-2.255 \times 10^{-3}$ & $-5.21 \times 10^{-5}$ & $\mathrm{o}$ \\
\hline 3.148 & $-6.468 \times 10^{-3}$ & $3.70 \times 10^{-3}$ & $-4.020 \times 10^{-3}$ & $1.93 \times 10^{-3}$ & o \\
\hline 3.165 & $-4.835 \times 10^{-3}$ & $1.47 \times 10^{-3}$ & $-3.005 \times 10^{-3}$ & $7.65 \times 10^{-4}$ & $6 \mathrm{p}^{6} \mathrm{~K}^{\mathrm{o}}$ \\
\hline 3.208 & $-6.537 \times 10^{-3}$ & $3.40 \times 10^{-3}$ & $-4.063 \times 10^{-3}$ & $1.77 \times 10^{-3}$ & $6 \mathrm{p}^{6} \mathrm{I}^{\mathrm{o}}$ \\
\hline 3.227 & $-4.928 \times 10^{-3}$ & $-6.67 \times 10^{-5}$ & $-3.063 \times 10^{-3}$ & $-3.48 \times 10^{-5}$ & o \\
\hline 3.319 & $-5.091 \times 10^{-3}$ & $2.60 \times 10^{-3}$ & $-3.164 \times 10^{-3}$ & $1.36 \times 10^{-3}$ & $6 \mathrm{p}^{6} \mathrm{I}^{\mathrm{o}}$ \\
\hline 3.337 & $-4.404 \times 10^{-3}$ & $-1.33 \times 10^{-3}$ & $-2.737 \times 10^{-3}$ & $-6.95 \times 10^{-4}$ & $6 \mathrm{p}^{6} \mathrm{~K}^{\mathrm{o}}$ \\
\hline 3.403 & $-4.174 \times 10^{-3}$ & $4.67 \times 10^{-4}$ & $-2.594 \times 10^{-3}$ & $2.43 \times 10^{-4}$ & $6 p^{o}$ \\
\hline 3.471 & $-5.273 \times 10^{-3}$ & $-3.67 \times 10^{-3}$ & $-3.278 \times 10^{-3}$ & $-1.91 \times 10^{-3}$ & $\mathrm{~T}$ \\
\hline 3.524 & $-4.053 \times 10^{-3}$ & $-3.14 \times 10^{-3}$ & $-2.519 \times 10^{-3}$ & $-1.63 \times 10^{-3}$ & $6 \mathrm{p}^{6} \mathrm{~K}^{\mathrm{o}}$ \\
\hline 3.578 & $-4.180 \times 10^{-3}$ & $-1.30 \times 10^{-3}$ & $-2.598 \times 10^{-3}$ & $-6.78 \times 10^{-4}$ & $6 \mathrm{p}^{6} \mathrm{I}^{\mathrm{o}}$ \\
\hline
\end{tabular}

Table C.11. HFS constants for nine out of the ten Sm II levels involved in the preselected transitions, for isotopes with non-zero nuclear spin.

\begin{tabular}{lrrrrr}
\hline \hline$E(\mathrm{eV})$ & \multicolumn{1}{c}{$A_{147}$} & \multicolumn{1}{c}{$B_{147}$} & \multicolumn{1}{c}{$A_{149}$} & \multicolumn{1}{c}{$B_{149}$} & Level \\
\hline 0.104 & $-1.058 \times 10^{-2}$ & $-2.37 \times 10^{-3}$ & $-8.719 \times 10^{-3}$ & $7.00 \times 10^{-4}$ & ${ }^{6} \mathrm{~s}^{8} \mathrm{~F}$ \\
0.185 & $-9.026 \times 10^{-3}$ & $-1.97 \times 10^{-3}$ & $-7.438 \times 10^{-3}$ & $5.67 \times 10^{-4}$ & $6 \mathrm{~s}^{8} \mathrm{~F}$ \\
0.378 & $-7.759 \times 10^{-3}$ & $2.74 \times 10^{-3}$ & $-6.394 \times 10^{-3}$ & $-8.01 \times 10^{-4}$ & $6 \mathrm{~s}^{8} \mathrm{~F}$ \\
0.544 & $2.845 \times 10^{-3}$ & $3.54 \times 10^{-3}$ & $2.345 \times 10^{-3}$ & $-1.03 \times 10^{-3}$ & $6 \mathrm{~s}^{6} \mathrm{~F}$ \\
0.659 & $2.151 \times 10^{-3}$ & $7.34 \times 10^{-3}$ & $1.775 \times 10^{-3}$ & $-2.13 \times 10^{-3}$ & $6^{6}{ }^{6} \mathrm{~F}$ \\
2.758 & $-6.401 \times 10^{-3}$ & $1.20 \times 10^{-3}$ & $-5.277 \times 10^{-3}$ & $-3.3 \times 10^{-4}$ & $6 \mathrm{p}^{\mathrm{o}}$ \\
2.932 & $-5.811 \times 10^{-3}$ & $3.67 \times 10^{-4}$ & $-4.787 \times 10^{-3}$ & $-1.0 \times 10^{-4}$ & $6 \mathrm{p}{ }^{\mathrm{o}}$ \\
3.049 & $-5.814 \times 10^{-3}$ & $1.13 \times 10^{-3}$ & $-4.793 \times 10^{-3}$ & $-3.3 \times 10^{-4}$ & $6 \mathrm{p}{ }^{\mathrm{o}}$ \\
3.174 & $-2.342 \times 10^{-3}$ & $7.34 \times 10^{-4}$ & $-1.931 \times 10^{-3}$ & $-2.0 \times 10^{-4}$ & $6 \mathrm{p}{ }^{\mathrm{o}}$ \\
\hline
\end{tabular}

Notes. The data are from Masterman et al. (2003).

Table C.12. HFS constants for seven Eu II levels.

\begin{tabular}{lrrrrll}
\hline \hline$E(\mathrm{eV})$ & \multicolumn{1}{c}{$A_{151}$} & \multicolumn{1}{c}{$B_{151}$} & \multicolumn{1}{c}{$A_{153}$} & \multicolumn{1}{c}{$B_{153}$} & Level & Reference \\
\hline 1.230 & -0.0178 & 0.0036 & -0.0079 & 0.0092 & a ${ }^{9} \mathrm{D}$ & $A$ : Hühnermann et al. (1992), B: Möller et al. (1993) \\
1.279 & -0.0038 & -0.0072 & -0.0017 & -0.0183 & $\mathrm{a}{ }^{9} \mathrm{D}$ & $A$ : Hühnermann et al. (1992), B: Möller et al. (1993) \\
1.320 & -0.0024 & 0.0007 & -0.0011 & 0.0020 & $\mathrm{a}{ }^{9} \mathrm{D}$ & Villemoes et al. (1992) \\
1.380 & -0.0024 & 0.0120 & -0.0011 & 0.0302 & $\mathrm{a}{ }^{9} \mathrm{D}$ & Villemoes et al. (1992) \\
3.245 & 0.0048 & 0.0155 & 0.0022 & 0.0399 & $\mathrm{z}{ }^{9} \mathrm{P}$ & Villemoes et al. (1992) \\
3.328 & 0.0019 & 0.0052 & 0.0008 & 0.0131 & $\mathrm{z}{ }^{7} \mathrm{P}$ & $A$ : Hühnermann et al. (1992), B: Möller et al. (1993) \\
3.361 & -0.0043 & -0.0166 & -0.0019 & -0.0425 & $\mathrm{z}{ }^{7} \mathrm{P}$ & $A$ : Hühnermann et al. (1992), B: Möller et al. (1993) \\
\hline
\end{tabular}




\section{Appendix D: Species occurring in the background line list only}

The background line list includes a number of lines for species which do not occur in the preselected line list. The references for the $g f$-values of those lines in the UVES-580 and GIRAFFE HR21 wavelength ranges with an estimated line depth in Arcturus greater than 1\% are given in Table D.1 (omitting species with less than five lines).

Table D.1. References for $g f$-values for species which occur only in the background line list.

\begin{tabular}{lclrl}
\hline \hline Name & $Z$ & Species & $N$ & References \\
\hline Phosphor & 15 & P I & 35 & KP, GUES, LAW, MRB \\
Potassium & 19 & K I & 40 & K12, WSM \\
Arsenic & 33 & As I & 10 & GUES \\
Rubidium & 37 & Rb I & 5 & GHR \\
Rhodium & 45 & Rh I & 70 & CB, DLb, KZBa, SDL \\
Palladium & 46 & Pd I & 20 & CB \\
Terbium & 65 & Tb II & 20 & MC, LWCS \\
Holmium & 67 & Ho I & 30 & MC \\
Erbium & 68 & Er I & 110 & MC \\
& & Er II & 80 & MC, LSCW, XJZD \\
Thulium & 69 & Tm I & 10 & MC, PK, WL \\
& & Tm II & 30 & QPB, WL \\
Ytterbium & 70 & Yb I & 15 & PGK, PK \\
& & Yb II & 40 & BDMQ \\
Lutetium & 71 & Lu I & 10 & FDLP, WV \\
& & Lu II & 15 & DCWL, QPBM \\
Hafnium & 72 & Hf I & 75 & CB, CBcor, DSLb \\
& & Hf II & 50 & CB, LDLS, LNWLX \\
Tantalum & 73 & Ta I & 140 & CB, CBcor, SDL \\
Rhenium & 75 & Re I & 40 & CB, DSLc \\
Osmium & 76 & Os I & 90 & CBcor, IAN, KZB \\
Iridium & 77 & Ir I & 25 & CBcor, GHcor \\
Platinum & 78 & Pt I & 20 & CB, DHL, GHLa, LGb \\
Thorium & 90 & Th I & 110 & MC \\
& & Th II & 205 & MC, NZL \\
\hline & & & &
\end{tabular}

Notes. $N$ is the approximate number of lines with an estimated line depth in Arcturus greater than 0.01 .

References. BDMQ: Biémont et al. (1998), CB: Corliss \& Bozman (1962), CBcor: Corliss \& Bozman (1962), DCWL: den Hartog et al. (1998), DLb: Duquette \& Lawler (1985), DHL: Den Hartog et al. (2005), DSLb: Duquette et al. (1982a), DSLc: Duquette et al. (1982b), FDLP: Fedchak et al. (2000), GHcor: Corliss \& Bozman (1962) data scaled to Gough et al. (1983), GHLa: Gough et al. (1982), GHR: von der Goltz et al. (1984), GUES: R. L. Kurucz, based on multiplet tables, IAN: Ivarsson et al. (2003), K12: Kurucz (2012), KP: Kurucz \& Peytremann (1975), KZB: Kwiatkowski et al. (1984), LAW: Lawrence (1967), LDLS: Lawler et al. (2007), LGb: Lotrian \& Guern (1982), LNWLX: Lundqvist et al. (2006), LSCW: Lawler et al. (2008b), LWCS: Lawler et al. (2001b), MC: Meggers et al. (1975), MRB: Miller et al. (1971), NZL: Nilsson et al. (2002), PGK: Penkin et al. (1984), PK: Penkin \& Komarovskii (1976), QPB: Quinet et al. (1999a), QPBM: Quinet et al. (1999b), SDL: Salih et al. (1983), WL: Wickliffe \& Lawler (1997b), WSM: Wiese et al. (1969), WV: Ward et al. (1985), XJZD: Xu et al. (2003c).

\section{Appendix E: Theoretical background for collisional broadening data}

We discuss three commonly used and presently available options for the calculation of spectral line broadening by collisions with ground state neutral hydrogen atoms. These are:

1. The Unsöld recipe. Lindholm-Foley theory employing the van der Waals long-range interaction potential $C_{6} / R^{6}$ as a function of internuclear distance $R$, where $C_{6}$ is calculated approximately as formulated by Unsöld (1955).

2. Kurucz calculations. Lindholm-Foley theory as in 1, where $C_{6}$ is calculated in more detail, as described by Kurucz (1981, p. 76).

3. The ABO theory. Detailed scattering calculations based on potentials from perturbation theory as described by Anstee \& O'Mara (1991), and successive expansions by P.S. Barklem and collaborators.

All three methods employ the impact theory (see for example Sect. 4.1 of Barklem 2016, and references therein), and thus the collisionally broadened line profile is described by a Lorentz profile. The long-range dispersion interaction between atoms is given by the van der Waals potential, which may be written in terms of the frequency shift $\Delta v=C_{6} / R^{6}$. In Lindholm-Foley theory (Lindholm 1946; Foley 1946), assuming such a form for the state-dependent dispersive part of the interaction that leads to line broadening, the line width (full width at half-maximum) is given in angular frequency units by

$\gamma=17.0 \cdot\left(C_{6, \text { upp }}-C_{6, \text { low }}\right)^{2 / 5} \cdot \bar{v}^{3 / 5} \cdot N_{\mathrm{H}}$,

where $\bar{v}$ is the mean relative speed, $N_{\mathrm{H}}$ the number density of hydrogen atom perturbers, and $C_{6 \text {,low }}$ and $C_{6 \text {,upp }}$ are the relevant constants for the interactions between the ground state hydrogen atoms and the atom of interest in the lower and upper states of the transition, respectively. The dispersion coefficient $C_{6}$ for the interaction between atoms A and B is given by (e.g. Margenau 1939, Sect. C.I.a)

$C_{6}=\frac{3}{2} \frac{e^{2} \hbar^{2}}{m_{e}} \sum_{k^{\prime} \neq k} \sum_{l^{\prime} \neq l} \frac{f_{k k^{\prime}}^{A} f_{l l^{\prime}}^{B}}{\left(E_{k}^{A}+E_{l}^{B}-E_{k^{\prime}}^{A}-E_{l^{\prime}}^{B}\right)\left(E_{k}^{A}-E_{k^{\prime}}^{A}\right)\left(E_{l}^{B}-E_{l^{\prime}}^{B}\right)}$,

where $E_{i}^{X}$ is the energy of level $i$ in atom $\mathrm{X}$, and $f_{i j}^{X}$ is the oscillator strength for the transition between levels $i$ and $j$, and the remaining symbols have their usual meaning.

If one of the atoms is a ground-state hydrogen atom, then the energy level separations are at least $10.2 \mathrm{eV}$, typically much larger than those in the other atom (especially for neutrals). Thus, the sums can be separated and the hydrogen atom part written in terms of the static dipole polarisability of hydrogen in its ground state $\alpha_{\mathrm{H}}$ (see Kurucz 1981, p. 76):

$C_{6, k}=\frac{e^{2} \alpha_{\mathrm{H}}}{h} \cdot \frac{3 \hbar^{2}}{2 m_{\mathrm{e}}} \sum_{k^{\prime}} \frac{f_{k k^{\prime}}}{E_{k}-E_{k^{\prime}}}$.

This is the expression used by Kurucz, and the sums are computed over all possible transitions (of the electrons in outer shells), using calculated $g f$-values calibrated by fits to observed energy levels. These sums are in general not complete, but they "should be complete or nearly so for most strong lines" 28 . Furthermore, for the calculation of the line width Eq. (E.1) is slightly

\footnotetext{
${ }^{28}$ See http://kurucz.harvard.edu/atoms/PROGRAMS/ expand3007. for
} 
modified - the maximum value of $C_{6, \text { upp }}$ and $C_{6 \text {,low }}$ is used instead of the difference.

The Unsöld recipe further approximates the summation over all states of the atom of interest by use of the completeness relation (noting that the oscillator strength is related by definition to the dipole operator), and the equation reduces to (Unsöld 1955)

$C_{6, k}=-\frac{e^{2} \alpha_{\mathrm{H}}}{h}\left\langle p^{2}\right\rangle$,

where $\left\langle p^{2}\right\rangle$ is the mean square radial coordinate of the optical electron on the atom of interest. The value of $\left\langle p^{2}\right\rangle$ can be estimated from the result in a hydrogenic atom, which gives $\left\langle p^{2}\right\rangle$ as a simple analytic expression as a function of the effective principle quantum number $n^{*}$ and the orbital angular momentum quantum number $l$ (see Bates \& Damgaard 1949, p. 111). Adopting this expression and neglecting the dependence on $l$ for simplicity, one obtains

$C_{6, k}=-\frac{e^{2} \alpha_{\mathrm{H}}}{h} \cdot 2.5 a_{0}^{2} \frac{n_{k}^{* 4}}{\left(Z-N_{\mathrm{e}}+1\right)^{2}}$,

where $a_{0}$ is the Bohr radius, $Z$ is the atomic number, and $N_{\mathrm{e}}$ is the total number of electrons for a given species (i.e. $Z-N_{\mathrm{e}}+1=$ $1,2, \ldots$ for neutral, singly ionised, ... species, respectively).

The effective principal quantum number is defined by the hydrogenic formula for the binding energy of the electron $\mathrm{nl}$, $E_{\mathrm{nl}}=-\left(Z-N_{\mathrm{e}}+1\right)^{2} /\left(n^{*}\right)^{2}$ Rydbergs. For a series of (core)- $n l$ levels, the binding energy of the electron is given by $E_{\mathrm{nl}}=E_{\text {limit }}-$ $E_{i}$, where $E_{\text {limit }}$ is the series limit energy for the appropriate core. For the case of a single excited electron outside a core in the ground state of the next higher ionisation stage, $E_{\text {limit }}$ is equal to the ionisation potential $E_{\text {ion }}$ of the absorbing species. However, the parent configuration of the state of interest may correspond to an excited state of the core. In that case, the energy of the excited core state $E P$ (relative to the core ground state) must be added to the ionisation potential when calculating $n^{*}$, that is, $E_{\text {limit }}=E_{\text {ion }}+E P$, in order to obtain the correct binding energy for the optical electron. Thus, for level $i$ with energy $E_{i}$, the effective principal quantum number should be calculated according to $n_{i}^{*}=\left(Z-N_{\mathrm{e}}+1\right)\left(\frac{E_{H}}{E_{\text {limit }}-E_{i}}\right)^{1 / 2}$, where $E_{\mathrm{H}}$ is the ionisation potential of hydrogen. Correct calculation of the binding energy can be very important in complex atoms such as $\mathrm{Ca}$ and Fe (see, e.g. Barklem et al. 2000a), but requires detailed information about the electron configurations. As far as we know, this has never been taken into account in the context of the Unsöld recipe, probably in part due to the fact that then simplicity of the calculation is largely lost, as one needs electron configuration information. In this work we adopt the same principle, and always adopt $E_{\text {limit }}=E_{\text {ion }}$ for the Unsöld recipe calculations; however, the correct values are adopted when employing the $\mathrm{ABO}$ theory, which is now described.

The ABO theory is a modification of the line-broadening theory by Brueckner (1971), and is described in Anstee \& O'Mara (1991). The main improvement with respect to theories based on the van der Waals potential is that interactions at intermediate internuclear distances are more accurately described. The calculation of the interaction energy does not employ the multipole expansion and thus is more appropriate for distances where the electronic wavefunctions overlap moderately. Intermediate-range interactions make an important contribution to the line-broadening cross sections. The second-most important improvement is that each $m$ state $(|m|=0,1, \ldots l)$ is treated, thus removing the averaging over phases found in the
Table E.1. Publications of line-broadening data from ABO theory and applicable ranges of effective principal quantum numbers for neutral species.

\begin{tabular}{lll}
\hline \hline Transition type & $\begin{array}{l}n^{*} \text { limits } \\
\text { lower ... upper }\end{array}$ & Reference \\
\hline s-p and p-s & s: $1.0 \ldots 3.0$ & Anstee \& O'Mara (1995) \\
& p: $1.3 \ldots 3.0$ & \\
p-d and d-p & d: $2.3 \ldots 4.0$ & Barklem \& O'Mara (1997) \\
d-f and f-d & f: $3.3 \ldots 5.0$ & Barklem et al. (1998) \\
\hline
\end{tabular}

Lindholm-Foley theory. ABO theory applies a model of the collision dynamics that includes the $m$-substates explicitly, and also includes the relative rotation of the atoms during the collision. Anstee \& O'Mara (1991) showed that neglect of these effects leads to an overestimate of the broadening by about $30 \%$. See Anstee \& O'Mara (1991) for more details, and Barklem (2016) for more references and a more detailed review of the differences between Lindholm-Foley and ABO theory.

For neutral species ABO theory can be applied to calculate cross-sections in general for transitions between different $l$ states. Cross-sections for transitions between $l=0,1,2,3$ (s, p, $\mathrm{d}, \mathrm{f}$, respectively) states are presented in the publications listed in Table E.1. These tabulate the line-broadening cross sections $\sigma$ for a specific value of the relative speed, $v_{0}=10^{4} \mathrm{~m} \mathrm{~s}^{-1}$, as a function of the effective principal quantum numbers $n^{*}$ of the relevant levels. The dependence of $\sigma$ on the collision speed $v$ is parametrised by a power-law exponent $\alpha$, which is also tabulated for the different $n^{*}$ combinations. We note that the ABO theory only applies to transitions that obey the one-electron selection rule $\Delta l= \pm 1$. However, in complex atoms one often finds situations where the optical electron (the outermost electron that determines the interaction potential) in the lower and upper states does not obey this rule, and one has transitions where the optical electron is in the same $l$ state throughout the transition, such as p-p. In these cases, since the upper state dominates the broadening, data can be estimated by taking a corresponding transition with the same upper state, for example $s-p$ instead of $p-p$.

Barklem et al. (2000b) determined effective principal quantum numbers, accounting correctly for core excitations, for 4872 lines of 23 neutral species from $\mathrm{Li}$ to $\mathrm{Ni}$ and obtained a list of broadening data by interpolation in the tables published earlier (see Table E.1). The list was constructed for all neutral species in the wavelength region between 230 and $1300 \mathrm{~nm}$, for which all of the required information was available in the NIST Atomic Spectra Database at that time, and for which the transition type and the $n^{*}$ values were within the table limits. An additional line selection criterion was $\log g f \geq-5$, as lines with lower $g f$-values were assumed to be unaffected by this type of broadening.

For ionised species it is not possible to tabulate the broadening data in a general way, while retaining the same accuracy as for neutrals. Broadening data were calculated for a few strong lines of Mg II and Ca II by Barklem \& O'Mara (1998) and of Be II, SrII, and Ba II by Barklem \& O'Mara (2000), and these data were included in Barklem et al. (2000b). Since then, over 24000 Fe II lines were calculated by Barklem \& Aspelund-Johansson (2005), and over $13000 \mathrm{Cr}$ II lines by P.S. Barklem $^{29}$.

${ }^{29}$ http://www.astro.uu.se/ barklem/data.html\#ad 
All of the published broadening data (cross sections $\sigma$ and velocity parameters $\alpha$ ) for specific lines of neutral and ionised species from the ABO data are made available through the VALD database. Using the speed dependence of $\sigma$ parametrised by $\alpha$ together with a Maxwell distribution, the integration over velocity leads to the following expression for line width:

$\gamma=2 \cdot(4 / \pi)^{\alpha / 2} \Gamma(2-\alpha / 2) v_{0} \sigma\left(v_{0}\right)\left(\bar{v} / v_{0}\right)^{1-\alpha} \cdot N_{\mathrm{H}}$,

where $\Gamma(x)$ is the Gamma function and $v_{0}=10^{4} \mathrm{~ms}^{-1}$.

Finally, we note that in all cases, collisions with ground state neutral helium can additionally be taken into account in an approximate way by replacing $N_{\mathrm{H}}$ with $N_{\mathrm{H}}+c N_{\mathrm{He}}$, where $N_{\mathrm{He}}$ is the number density of helium atoms. The coefficient $c \approx 0.41$ accounts for differences in the polarisability and for the mass (and thus mean velocity) of $\mathrm{He}$.

\section{Appendix F: Broadening data comparisons}

The ABO theory has been extensively tested through comparisons with the solar spectrum (e.g. all papers in Table E.1, as well as Barklem \& O'Mara 2001) and with more detailed calculations where available (e.g. Kerkeni et al. 2004). Based on these comparisons the ABO theory is estimated to be accurate to better than 20 to $30 \%$, though in some cases it may be significantly better (see Anstee \& O'Mara 1995). On the other hand, the Unsöld recipe, based on the Lindholm-Foley theory and the van der Waals interaction, is well-known to be typically only reliable to about a factor of 2. See Barklem (2016) for a more detailed discussion and comparison of the various theories and the evidence for their accuracies. However, the conclusion is that the evidence indicates that the ABO theory is the most reliable of the three methods considered here.

In Fig. F.1 we compare line widths computed with the three different options described in Appendix E for lines of a representative neutral species-Fe I-at a representative temperature of $6000 \mathrm{~K}$. Lines for which both Kurucz calculations and ABO data are available were extracted from the VALD database in the wavelength range 480 to $680 \mathrm{~nm}$ (on 2 Jul 2012). The figure shows the ratio of the ABO width to the width from the Kurucz line list and to the Unsöld width (the latter calculated from Eqs. (E.5) and (E.1)). On the basis that the ABO value represents the most realistic line width, the ratio can be seen as an 'enhancement factor' to be applied to the Kurucz or Unsöld $\gamma$-values. The ratio is shown as a function of lower level energy, and different symbols represent different transition types (combinations of $l$ quantum numbers).

A large spread in the ratios is evident for high values of the excitation energy $\left(E_{\text {low }} \gtrsim 2.8 \mathrm{eV}\right.$ ), for both Kurucz and Unsöld values. For $\mathrm{p}-\mathrm{s}$ and $\mathrm{p}-\mathrm{d}$ transitions the Kurucz ratios cluster around 2, while the Unsöld ratios cluster at two different values (close to 2.5 for $\mathrm{p}-\mathrm{s}$ and 1.5 for $\mathrm{p}-\mathrm{d}$ ), and a few $\mathrm{p}-\mathrm{s}$ and $\mathrm{p}-\mathrm{d}$ lines are seen at 1.5 and 0.5 , respectively. For the $s-p$ transitions with $E_{\text {low }} \gtrsim 2.8 \mathrm{eV}$ all Unsöld ratios are less than 1, implying that a 'reduction factor' would be required for Unsöld values with respect to $\mathrm{ABO}$ theory. Low $\mathrm{ABO}$ values are obtained when the transition takes place involving one or two levels with excited cores. In such a case, the value of $E_{\text {limit }}$ in the calculation of the effective principal quantum number $n^{*}$ is increased with respect to if $E_{\text {ion }}$ is used (as typically done in the Unsöld recipe), which implies a smaller $n^{*}$ value and hence a decreased $\sigma$. On the other hand, the Kurucz ratios cluster around 1 and 1.5. Finally, for lines with $E_{\text {low }}<2.8 \mathrm{eV}$ (s-p transitions) the Kurucz ratios gradually

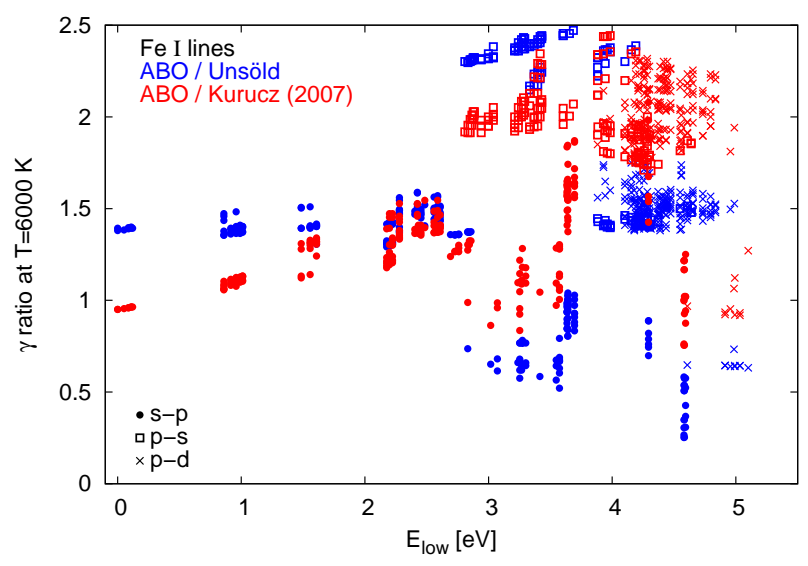

Fig. F.1. Ratio of collisional line width $\gamma$ calculated from ABO theory to line width from van der Waals theory using Kurucz or Unsöld $C_{6}$ values, for Fe I lines of transition types $\mathrm{s}-\mathrm{p}, \mathrm{p}-\mathrm{s}$, and $\mathrm{p}-\mathrm{d}$, at a temperature of $6000 \mathrm{~K}$, as a function of lower level energy $E_{\text {low }}$.
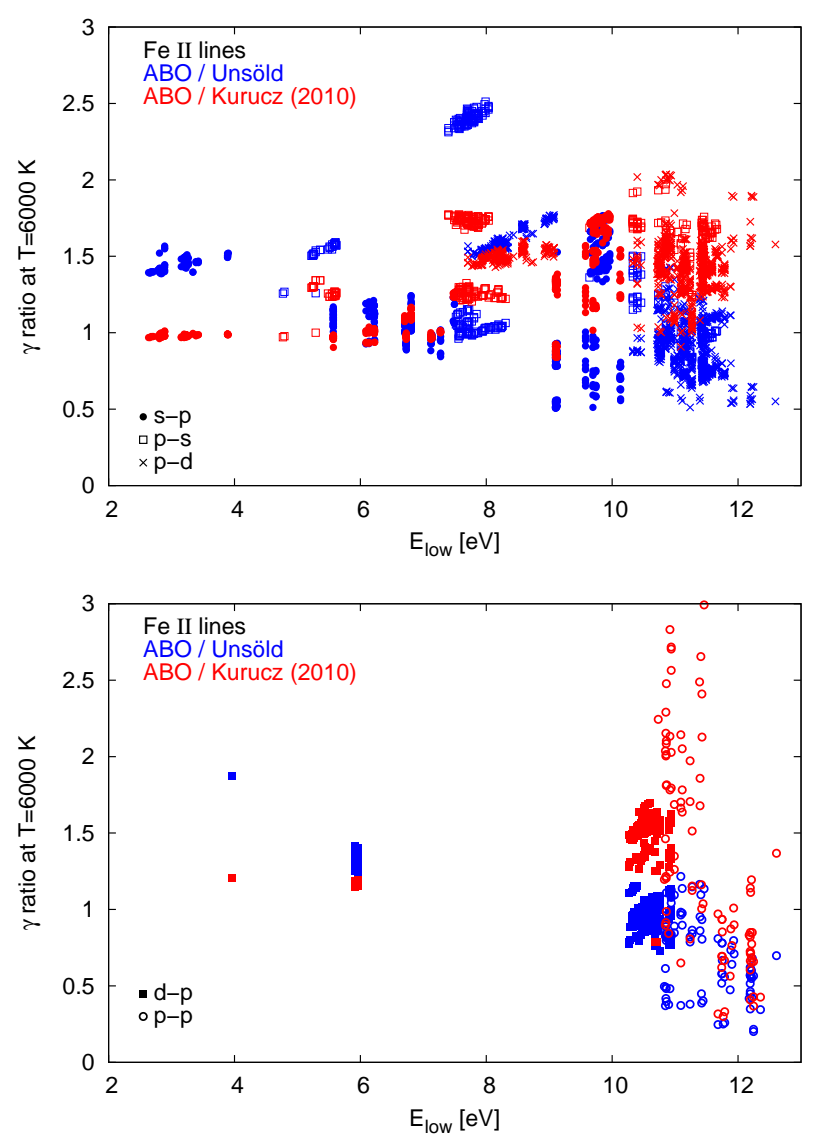

Fig. F.2. Ratio of collisional line width $\gamma$ calculated from ABO theory to line width from van der Waals theory using Kurucz or Unsöld $C_{6}$ values, for $\mathrm{Fe}$ II lines of transition types $\mathrm{s}-\mathrm{p}, \mathrm{p}-\mathrm{s}, \mathrm{p}-\mathrm{d}$ (upper panel), $\mathrm{d}-\mathrm{p}$ and $\mathrm{p}-\mathrm{p}$ (lower panel), at a temperature of $6000 \mathrm{~K}$, as a function of lower level energy $E_{\text {low }}$.

increase from 1 to 1.5 , while the Unsöld ratios are constant at around 1.5 .

Figure F.2 shows a similar comparison for lines of a representative ionised species-Fe II. We see an equally complex pattern of enhancements of line widths, in particular for Unsöld values at high excitation energies $\left(E_{\text {low }} \gtrsim 7.3 \mathrm{eV}\right)$. Kurucz ratios are close to 1 for $E_{\text {low }}<7.3 \mathrm{eV}$ (all of these are s-p, p-s, or d-p transitions). 


\section{Appendix G: Broadening data for preselected lines}

\section{G.1. Neutral species}

In view of the comparisons presented in Appendix $F$ it seems impossible to define a universal enhancement factor for broadening data calculated with the Unsöld recipe. Thus, it is desirable to use $\mathrm{ABO}$ theory data whenever possible. $\mathrm{ABO}$ data were available from Barklem et al. (2000b) for about $70 \%$ of the preselected lines in the Gaia-ESO line list (see Sect. 2.1 and Appendix B for a description of the preselected line list). We aimed at increasing the percentage at least for the lines with otherwise high-quality data. New ABO data were determined for most of the $Y / Y$ lines and a few $Y / U, U / Y$, and $U / U$ lines lacking such data. These were mostly lines of Fe I, and a few lines of $\mathrm{C} I$, Si I, Ca I, Sc I, Cr I, Zn I, Zr I, and Mo I.

For these lines we extracted the atomic data from the NIST Atomic Spectra Database required to calculate the effective principal quantum number $n^{*}$. In particular, term designations and electron configurations of the levels involved in the transitions were obtained. We also determined the electron configuration of the parent terms and checked whether they deviated from the ground states of the corresponding singly ionised species listed in Table G.1. In that case the excitation energy of the parent term was determined and added to the ionisation energy in the calculation of the effective principal quantum number. All of these input data are given in Table G.2.

We then interpolated in the cross-section tables of the ABO publications listed in Table E.1, where possible. The interpolation was done using the code widthcomp (version 2.0) from the abo-cross package (Barklem et al. 2015). For a few lines, the $n^{*}$ values (given in Table G.3) fall outside the range of the relevant cross-section interpolation table. In these cases the cross sections were calculated individually for each line. For example, this occurs for eight of the Fe I lines which are $\mathrm{d}-\mathrm{p}$ transitions. In all cases both states have a parent configuration corresponding to an excited state of Fe II (see Table G.2), resulting in $n_{d}^{*} \approx 1.8$, and $n_{p}^{*} \lesssim 1.9$ (see Table G.3). The cross sections calculated directly for these lines are about 300-400 atomic units. The results for all lines are given in Table G.3. In total, we determined new ABO values for 41 lines: $28 \mathrm{Fe}$ lines $(\mathrm{s}-\mathrm{p}, \mathrm{p}-\mathrm{s}, \mathrm{p}-\mathrm{d}, \mathrm{d}-\mathrm{p})$, two $\mathrm{C}$ lines ( $\mathrm{p}-\mathrm{p}, \mathrm{p}-\mathrm{d})$, three, two, and one $\mathrm{s}-\mathrm{p}$ lines of $\mathrm{Si}, \mathrm{Sc}$, and $\mathrm{Cr}$, respectively, and five lines for elements with $Z>28$ (s-p and $\mathrm{p}-\mathrm{s}$ lines
Table G.1. Ionisation energies $E_{\text {ion }}$ of neutral species listed in Tables G.2 and G.3 and ground states of corresponding singly ionised species (Martin et al. 2011; Ralchenko et al. 2011).

\begin{tabular}{rlrrr}
\hline \hline$Z$ & Element & $E_{\text {ion }}(\mathrm{eV})$ & Configuration & Term \\
\hline 6 & $\mathrm{C}$ & 11.2603 & $2 \mathrm{~s}^{2} 2 \mathrm{p}$ & ${ }^{2} \mathrm{P}^{o}$ \\
14 & $\mathrm{Si}$ & 8.1517 & $3 \mathrm{~s}^{2} 3 \mathrm{p}$ & ${ }^{2} \mathrm{P}^{o}$ \\
20 & $\mathrm{Ca}$ & 6.1132 & $3 \mathrm{p}^{6} 4 \mathrm{~s}$ & ${ }^{2} \mathrm{~S}$ \\
21 & $\mathrm{Sc}$ & 6.5615 & $3 \mathrm{p}^{6} 3 \mathrm{~d} 4 \mathrm{~s}$ & ${ }^{3} \mathrm{D}$ \\
24 & $\mathrm{Cr}$ & 6.7665 & $3 \mathrm{~d}^{5}$ & $\mathrm{a}^{6} \mathrm{~S}$ \\
26 & $\mathrm{Fe}$ & 7.9024 & $3 \mathrm{~d}^{6}\left({ }^{5} \mathrm{D}\right) 4 \mathrm{~s}$ & $a^{6} \mathrm{D}$ \\
30 & $\mathrm{Zn}$ & 9.3942 & $3 \mathrm{~d}^{10} 4 \mathrm{~s}$ & ${ }^{2} \mathrm{~S}$ \\
40 & $\mathrm{Zr}$ & 6.6339 & $4 \mathrm{~d}^{2}\left({ }^{3} \mathrm{~F}\right) 5 \mathrm{~s}$ & $a^{4} \mathrm{~F}$ \\
42 & $\mathrm{Mo}$ & 7.0924 & $4 \mathrm{~d}^{5}$ & $\mathrm{a}^{6} \mathrm{~S}$ \\
\hline
\end{tabular}

of $\mathrm{Zn}, \mathrm{Zr}$, and $\mathrm{Mo}$ ). For the two $\mathrm{Ca}$ lines it was not possible to determine reliable $\mathrm{ABO}$ values.

\section{G.2. Ionised species}

All lines of $\mathrm{Cr}$ II and $\mathrm{Fe}$ II in the preselected line list have $\mathrm{ABO}$ data (Barklem \& Aspelund-Johansson 2005). All four Ba II lines (Appendix B.27) have ABO data (Barklem \& O’Mara 1998, 2000) as well. For CaII (Appendix B.10) only the three NIR triplet lines have ABO data (Barklem \& O'Mara 1998). For the other preselected Ca II lines calculations by Kurucz (2010b) are available. These are $\mathrm{p}-\mathrm{d}, \mathrm{d}-\mathrm{f}$, and $\mathrm{f}-\mathrm{g}$ transitions with rather high excitation potentials $\left(E_{\text {low }}>7 \mathrm{eV}\right)$.

No ABO theory calculations are available for the remaining ionised species. For the two preselected Si II lines (s-p transitions with $E_{\text {low }}=8.1 \mathrm{eV}$ ), calculations by Kurucz (2012) are available. For the lines of Sc II (s-p, d-s), Ti II (s-p, d-p), and Y II (d-p), calculations by Kurucz $(2009,2010 b, 2011)$ are available, respectively. The remaining species and transitions have no data. These are Zr II (s-p, d-p) and the rare-earth elements La II (s-d, d-d), Ce II (s-p, d-p, d-d), Pr II (d-p, d-d), Nd II (s-s, s-d, s-?, d-p, d-d, d-?), Sm II (s-s, s-p), Eu II (d-p), Gd II (s-s), and Dy II $(s-p)$. Here "?" means that there are some transitions for which the upper level $l$ state is unknown. All lines of the ionised species without $\mathrm{ABO}$ data (except for $\mathrm{Si}$ II and $\mathrm{Ca}$ II) have low excitation potentials $\left(E_{\text {low }} \lesssim 3 \mathrm{eV}\right)$. 
Table G.2. Atomic data for high-quality lines of neutral species in the preselected Gaia-ESO line list which are not included in Barklem et al. (2000b).

\begin{tabular}{|c|c|c|c|c|c|c|c|c|c|}
\hline$Z$ & El & Wavelength $(\AA)$ & $\mathrm{T}_{\text {low }}$ & $\mathrm{T}_{\text {upp }}$ & Type & $\mathrm{PT}_{\text {low }}$ & $\mathrm{EP}_{\text {low }}(\mathrm{eV})$ & $\mathrm{PT}_{\mathrm{upp}}$ & $\mathrm{EP}_{\text {upp }}(\mathrm{eV})$ \\
\hline 6 & $\mathrm{C}$ & 6587.6100 & ${ }^{1} P$ & ${ }^{1} P^{o}$ & $\mathrm{p}-\mathrm{d}$ & ${ }^{2} P^{o}$ & 0 & ${ }^{2} P^{o}$ & 0 \\
\hline 6 & $\mathrm{C}$ & 8727.1260 & ${ }^{1} D$ & ${ }^{1} S$ & $\mathrm{p}-\mathrm{p}$ & ${ }^{2} P^{o}$ & 0 & ${ }^{2} P^{o}$ & 0 \\
\hline 14 & $\mathrm{Si}$ & 5690.4250 & ${ }^{3} P^{o}$ & ${ }^{3} P$ & $\mathrm{~s}-\mathrm{p}$ & ${ }^{2} P^{o}$ & 0 & ${ }^{2} P^{o}$ & 0 \\
\hline 14 & $\mathrm{Si}$ & 5701.1040 & ${ }^{3} P^{o}$ & ${ }^{3} P$ & $\mathrm{~s}-\mathrm{p}$ & ${ }^{2} P^{o}$ & 0 & ${ }^{2} P^{o}$ & 0 \\
\hline 14 & $\mathrm{Si}$ & 5793.0730 & ${ }^{3} P^{o}$ & ${ }^{3} D$ & $\mathrm{~s}-\mathrm{p}$ & ${ }^{2} P^{o}$ & 0 & ${ }^{2} P^{o}$ & 0 \\
\hline 20 & $\mathrm{Ca}$ & 5512.9800 & ${ }^{1} P$ & ${ }^{1} S$ & $\mathrm{p}-\mathrm{p}$ & ${ }^{2} S$ & 0 & ${ }^{2} S$ & 0 \\
\hline 20 & $\mathrm{Ca}$ & 5867.5620 & ${ }^{1} P$ & ${ }^{1} S$ & $\mathrm{p}-\mathrm{s}$ & ${ }^{2} S$ & 0 & ${ }^{2} S$ & 0 \\
\hline 21 & $\mathrm{Sc}$ & 5356.0910 & ${ }^{2} F$ & ${ }^{2} D^{o}$ & $\mathrm{~s}-\mathrm{p}$ & ${ }^{3} F$ & 0.595480 & ${ }^{3} F$ & 0.595480 \\
\hline 21 & $\mathrm{Sc}$ & 5484.6260 & ${ }^{2} F$ & ${ }^{2} F^{o}$ & $\mathrm{~s}-\mathrm{p}$ & ${ }^{3} F$ & 0.595480 & ${ }^{3} F$ & 0.595480 \\
\hline 24 & $\mathrm{Cr}$ & 5788.3820 & $b^{5} D$ & $y^{5} D^{o}$ & $\mathrm{~s}-\mathrm{p}$ & $b^{4} D$ & 3.103779 & $a^{6} D$ or $a^{4} D$ & $1.95213^{(a)}$ \\
\hline 26 & $\mathrm{Fe}$ & 4802.8797 & $b^{3} D$ & $w^{3} P^{o}$ & $\mathrm{~s}-\mathrm{p}$ & $a^{6} D$ & 0 & $a^{2} P$ & 2.2764 \\
\hline 26 & $\mathrm{Fe}$ & 4962.5719 & $y^{5} F^{o}$ & $e^{3} H$ & $\mathrm{p}-\mathrm{d}$ & $a^{4} F$ & 0.2322 & $a^{4} F$ & 0.2322 \\
\hline 26 & $\mathrm{Fe}$ & 4985.2529 & $z^{3} D^{o}$ & $e^{3} D$ & $\mathrm{p}-\mathrm{s}$ & $a^{6} D$ & 0 & $a^{4} D$ & 0.9863 \\
\hline 26 & $\mathrm{Fe}$ & 5060.0780 & $a^{5} D$ & $z^{7} D^{o}$ & $s-p$ & $a^{6} D$ & 0 & $a^{6} D$ & 0 \\
\hline 26 & $\mathrm{Fe}$ & 5293.9588 & $c^{3} F$ & $u^{3} D^{o}$ & $\mathrm{~d}-\mathrm{p}$ & $a^{4} F$ & 0.2322 & $a^{2} P$ & 2.2764 \\
\hline 26 & $\mathrm{Fe}$ & 5365.3990 & $a^{1} H$ & $z^{1} G^{o}$ & $\mathrm{~s}-\mathrm{p}$ & $a^{2} H$ & 2.5219 & $a^{2} G$ & 1.9645 \\
\hline 26 & $\mathrm{Fe}$ & 5464.2796 & $c^{3} F$ & $y^{1} D^{o}$ & $d-p$ & $a^{4} F$ & 0.2322 & $a^{2} P$ & 2.2764 \\
\hline 26 & $\mathrm{Fe}$ & 5494.4626 & $c^{3} F$ & $x^{3} H^{o}$ & $d-p$ & $a^{4} F$ & 0.2322 & $a^{4} G$ or $b^{2} G$ & $3.4602^{(a)}$ \\
\hline 26 & $\mathrm{Fe}$ & 5655.1760 & $x^{5} F^{o}$ & $g^{5} G$ & $\mathrm{p}-\mathrm{d}$ & $a^{6} D$ & 0 & $a^{6} D$ & 0 \\
\hline 26 & $\mathrm{Fe}$ & 5662.5160 & $y^{5} F^{o}$ & $g^{5} D$ & $\mathrm{p}-\mathrm{s}$ & $a^{4} F$ & 0.2322 & $a^{4} D$ & 0.9863 \\
\hline 26 & $\mathrm{Fe}$ & 5775.0806 & $y^{5} F^{o}$ & $g^{5} D$ & $\mathrm{p}-\mathrm{s}$ & $a^{4} F$ & 0.2322 & $a^{4} D$ & 0.9863 \\
\hline 26 & $\mathrm{Fe}$ & 5853.1483 & $a^{3} F$ & $z^{5} P^{o}$ & $s-p$ & $a^{4} F$ & 0.2322 & $a^{6} D$ & 0 \\
\hline 26 & $\mathrm{Fe}$ & 6027.0509 & $c^{3} F$ & $v^{3} G^{o}$ & $d-p$ & $a^{4} F$ & 0.2322 & $a^{2} G$ & 1.9645 \\
\hline 26 & $\mathrm{Fe}$ & 6120.2460 & $a^{5} F$ & $z^{7} P^{o}$ & $\mathrm{~s}-\mathrm{p}$ & $a^{4} F$ & 0.2322 & $a^{6} D$ & 0 \\
\hline 26 & $\mathrm{Fe}$ & 6127.9060 & $c^{3} F$ & $y^{3} H^{o}$ & $d-p$ & $a^{4} F$ & 0.2322 & $a^{2} G$ & 1.9645 \\
\hline 26 & $\mathrm{Fe}$ & 6157.7279 & $c^{3} F$ & $w^{3} F^{o}$ & $d-p$ & $a^{4} F$ & 0.2322 & $a^{2} G$ & 1.9645 \\
\hline 26 & $\mathrm{Fe}$ & 6165.3600 & $c^{3} F$ & $v^{3} G^{o}$ & $\mathrm{~d}-\mathrm{p}$ & $a^{4} F$ & 0.2322 & $a^{2} G$ & 1.9645 \\
\hline 26 & $\mathrm{Fe}$ & 6301.5000 & $z^{5} P^{o}$ & $e^{5} D$ & $\mathrm{p}-\mathrm{s}$ & $a^{6} D$ & 0 & $a^{6} D$ & 0 \\
\hline 26 & $\mathrm{Fe}$ & 6315.8110 & $c^{3} F$ & $y^{1} G^{o}$ & $d-p$ & $a^{4} F$ & 0.2322 & $b^{2} H$ & 3.2447 \\
\hline 26 & $\mathrm{Fe}$ & 6380.7430 & $c^{3} F$ & $w^{3} F^{o}$ & $d-p$ & $a^{4} F$ & 0.2322 & $a^{2} G$ & 1.9645 \\
\hline 26 & $\mathrm{Fe}$ & 6400.3170 & $a^{5} F$ & $z^{7} F^{o}$ & $s-p$ & $a^{4} F$ & 0.2322 & $a^{6} D$ & 0 \\
\hline 26 & $\mathrm{Fe}$ & 6574.2270 & $a^{5} F$ & $z^{7} F^{o}$ & $\mathrm{~s}-\mathrm{p}$ & $a^{4} F$ & 0.2322 & $a^{6} D$ & 0 \\
\hline 26 & $\mathrm{Fe}$ & 6648.0800 & $a^{5} F$ & $z^{7} F^{o}$ & $s-p$ & $a^{4} F$ & 0.2322 & $a^{6} D$ & 0 \\
\hline 26 & $\mathrm{Fe}$ & 8515.1084 & $b^{3} G$ & $z^{3} G^{o}$ & $s-p$ & $a^{6} D$ & 0 & $a^{4} F$ & 0.2322 \\
\hline 26 & $\mathrm{Fe}$ & 8582.2574 & $b^{3} G$ & $z^{3} G^{o}$ & $s-p$ & $a^{6} D$ & 0 & $a^{4} F$ & 0.2322 \\
\hline 26 & $\mathrm{Fe}$ & 8621.6007 & $b^{3} G$ & $z^{3} G^{o}$ & $s-p$ & $a^{6} D$ & 0 & $a^{4} F$ & 0.2322 \\
\hline 26 & $\mathrm{Fe}$ & 8699.4540 & $x^{5} D^{o}$ & $f^{5} F$ & $\mathrm{p}-\mathrm{d}$ & $a^{6} D$ & 0 & $a^{6} D$ & 0 \\
\hline 26 & $\mathrm{Fe}$ & 8922.6500 & $x^{5} F^{o}$ & $f^{5} F$ & $\mathrm{p}-\mathrm{d}$ & $a^{6} D$ & 0 & $a^{6} D$ & 0 \\
\hline 30 & $\mathrm{Zn}$ & 4810.5280 & ${ }^{3} P^{o}$ & ${ }^{3} S$ & $\mathrm{p}-\mathrm{s}$ & ${ }^{2} S$ & 0 & ${ }^{2} S$ & 0 \\
\hline 40 & $\mathrm{Zr}$ & 6127.4750 & $a^{3} F$ & $z^{3} F^{o}$ & $\mathrm{~s}-\mathrm{p}$ & $a^{4} F$ & 0 & $a^{4} F$ & 0 \\
\hline 40 & $\mathrm{Zr}$ & 6134.5850 & $a^{3} F$ & $z^{3} F^{o}$ & $s-p$ & $a^{4} F$ & 0 & $a^{4} F$ & 0 \\
\hline 40 & $\mathrm{Zr}$ & 6140.5350 & $a^{3} P$ & $z^{3} P^{o}$ & $\mathrm{~s}-\mathrm{p}$ & $a^{4} F$ & 0 & $a^{4} F$ & 0 \\
\hline 42 & Mo & 6030.6440 & $a^{5} D$ & $z^{5} P^{o}$ & $\mathrm{~s}-\mathrm{p}$ & $a^{6} D$ & 1.460950 & $a^{6} S$ & 0 \\
\hline
\end{tabular}

Notes. $\mathrm{T}_{\text {low }}$ and $\mathrm{T}_{\text {upp }}$ are the terms of the lower and upper levels. Type is the transition type, indicated by the orbital angular momentum quantum numbers of the optical electrons. $\mathrm{PT}_{\text {low }}$ and $\mathrm{PT}_{\text {upp }}$ are the parent terms of the lower and upper levels (to be compared with Table G.1), with excitation energies $\mathrm{EP}_{\text {low }}$ and $\mathrm{EP}_{\text {upp }}$. Term designations are from Ralchenko et al. (2011). Level energies and rotational quantum numbers can be found in the general line-list table. ${ }^{(a)}$ Average energy of the two possible terms. 
Table G.3. Effective principal quantum numbers $n^{*}$, and ABO theory broadening data, for high-quality lines of neutral species, which are not included in Barklem et al. (2000b).

\begin{tabular}{|c|c|c|c|c|c|c|c|}
\hline$Z$ & $\mathrm{El}$ & $\begin{array}{r}\text { Wavelength } \\
(\AA)\end{array}$ & $n_{\text {low }}^{*}$ & $n_{\text {upp }}^{*}$ & $\begin{array}{r}\sigma \\
\left(a_{0}^{2}\right) \\
\end{array}$ & $\alpha$ & \\
\hline 6 & $\mathrm{C}$ & 6587.6100 & 2.23 & 4.02 & 1953 & 0.320 & \\
\hline 6 & $\mathrm{C}$ & 8727.1260 & 1.17 & 1.26 & 154 & 0.266 & $a$ \\
\hline 14 & $\mathrm{Si}$ & 5690.4250 & 2.05 & 3.61 & 1770 & 0.220 & $b$ \\
\hline 14 & $\mathrm{Si}$ & 5701.1040 & 2.05 & 3.60 & 1770 & 0.220 & $b$ \\
\hline 14 & $\mathrm{Si}$ & 5793.0730 & 2.05 & 3.54 & 1700 & 0.230 & $b$ \\
\hline 20 & $\mathrm{Ca}$ & 5512.9800 & 2.07 & 3.83 & & & $c$ \\
\hline 20 & $\mathrm{Ca}$ & 5867.5620 & 2.07 & 3.57 & & & $d$ \\
\hline 21 & $\mathrm{Sc}$ & 5356.0910 & 1.603 & 2.137 & 412 & 0.271 & \\
\hline 21 & $\mathrm{Sc}$ & 5484.6260 & 1.601 & 2.113 & 401 & 0.281 & \\
\hline 24 & $\mathrm{Cr}$ & 5788.3820 & 1.408 & 1.953 & 316 & 0.264 & \\
\hline 26 & $\mathrm{Fe}$ & 4962.5719 & 1.854 & 3.052 & 905 & 0.278 & \\
\hline 26 & $\mathrm{Fe}$ & 4802.8797 & 1.794 & 1.862 & 356 & 0.244 & \\
\hline 26 & $\mathrm{Fe}$ & 4985.2529 & 1.857 & 2.360 & 742 & 0.240 & \\
\hline 26 & $\mathrm{Fe}$ & 5060.0780 & 1.312 & 1.579 & 205 & 0.252 & \\
\hline 26 & $\mathrm{Fe}$ & 5293.9588 & 1.846 & 1.918 & 290 & 0.250 & $b$ \\
\hline 26 & $\mathrm{Fe}$ & 5365.3990 & 1.412 & 1.855 & 283 & 0.261 & \\
\hline 26 & $\mathrm{Fe}$ & 5464.2796 & 1.846 & 1.900 & 380 & 0.250 & $b$ \\
\hline 26 & $\mathrm{Fe}$ & 4626 & 1.8 & 1.6 & 330 & 90 & 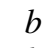 \\
\hline 26 & $\mathrm{Fe}$ & 760 & 2.189 & 4.583 & 2010 & 0.250 & 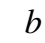 \\
\hline 26 & $\mathrm{Fe}$ & 5662.5160 & 1.862 & 2.337 & 724 & 0.235 & \\
\hline 26 & $\mathrm{Fe}$ & 5775.0806 & 1.872 & 2.337 & 720 & 0.231 & \\
\hline 26 & $\mathrm{Fe}$ & 5853.1483 & 1.430 & 1.778 & 264 & 0.242 & \\
\hline 26 & $\mathrm{Fe}$ & 6027.0509 & 1.830 & 1.908 & 380 & 0.250 & $U$ \\
\hline 26 & $\mathrm{Fe}$ & 6120.2460 & 1.376 & 1.661 & 229 & 0.252 & 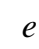 \\
\hline 26 & $\mathrm{Fe}$ & 6127.9060 & 1.846 & 1.917 & 290 & 0.250 & $b$ \\
\hline 26 & $\mathrm{Fe}$ & 6157.7279 & 1.830 & 1.897 & 375 & 0.255 & $b$ \\
\hline 26 & $\mathrm{Fe}$ & 6165.3600 & 1.846 & 1.914 & 380 & 0.250 & 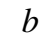 \\
\hline 26 & $\mathrm{Fe}$ & 6301.5000 & 1.789 & 2.441 & 832 & 0.243 & \\
\hline 26 & $\mathrm{Fe}$ & 6315.8110 & 1.830 & 1.682 & 410 & 0.250 & $b$ \\
\hline 26 & $\mathrm{Fe}$ & 6380.7430 & 1.856 & 1.907 & 380 & 0.250 & b \\
\hline 26 & $\mathrm{Fe}$ & 6400.3170 & 1.376 & 1.646 & 226 & 0.253 & \\
\hline 26 & $\mathrm{Fe}$ & 6574.2270 & 1.380 & 1.645 & 227 & 0.254 & \\
\hline 26 & $\mathrm{Fe}$ & 6648.0800 & 1.385 & 1.650 & 229 & 0.254 & $e$ \\
\hline 26 & $\mathrm{Fe}$ & 8515.1084 & 1.674 & 1.936 & 356 & 0.242 & \\
\hline 26 & $\mathrm{Fe}$ & 8582.2574 & 1.669 & 1.925 & 352 & 0.244 & \\
\hline 26 & $\mathrm{Fe}$ & 8621.6007 & 1.662 & 1.913 & 347 & 0.246 & \\
\hline 26 & $\mathrm{Fe}$ & 8699.4540 & 2.148 & 2.989 & 817 & 0.272 & \\
\hline 26 & $\mathrm{Fe}$ & 8922.6500 & 2.173 & 3.022 & 839 & 0.278 & \\
\hline 30 & $\mathrm{Zn}$ & 4810.5280 & 1.599 & 2.228 & 676 & 0.238 & \\
\hline 40 & $\mathrm{Zr}$ & 6127.4750 & 1.449 & 1.747 & 260 & 0.244 & \\
\hline 40 & $\mathrm{Zr}$ & 6134.5850 & 1.432 & 1.717 & 251 & 0.248 & \\
\hline 40 & $\mathrm{Zr}$ & 6140.5350 & 1.491 & 1.822 & 287 & 0.242 & \\
\hline 42 & Mo & 6030.6440 & 1.392 & 1.969 & 322 & 0.262 & \\
\hline
\end{tabular}

Notes. ABO theory broadening data are given as cross section $\sigma$ in atomic units and velocity exponent $\alpha$. Broadening data were either interpolated in the tables of Anstee \& O'Mara (1995) and Barklem \& O'Mara (1997) or calculated by PSB. (a) p-p transition; s-p table was used; $\log g f=-8$. (b) New calculation or estimate by PSB. (c) p-p transition; outside range of s-p table. $(d) \sigma=2170$ a.u. at $v=10^{4} \mathrm{~m} \mathrm{~s}^{-1}$; $\alpha$ could not be determined because of poor dependence of $\sigma$ on $v$. (e) $\log g f<-5$.

\section{Appendix H: Recommendations and conclusions for collisional broadening data}

For neutral species in the preselected line list, we recommend to use the ABO theory cross-sections provided with the GaiaESO line list. These include the newly derived values given in Table G.3 and should be used with Eq. (E.6) to compute line widths. The number of lines with $\mathrm{ABO}$ data per species and for various quality classifications is given in Table 1 . For future applications of the line list, the ABO theory interpolation work should be extended to the remaining preselected neutral lines. These are $103 \mathrm{Fe}$ I lines and about 200 lines of other species (e.g. $42 \mathrm{Si}$ I and $21 \mathrm{Ti}$ I lines, and about ten lines each of neutral $\mathrm{Na}$, $\mathrm{Mg}, \mathrm{S}, \mathrm{Cr}, \mathrm{Mn}, \mathrm{Co}, \mathrm{Ni}, \mathrm{Sr}, \mathrm{Zr}, \mathrm{Nb})$.

For ionised species Fig. F.2 indicates that broadening calculations by Kurucz are close to the ABO theory data for low excitation energies. Although we do not know the limit for $E_{\text {low }}$ for species other than Fe II, we recommend to use Kurucz data for the preselected Sc II, Ti II, and Y II lines, based on their rather low $E_{\text {low }}$ values ${ }^{30}$. For the rare-earth species we recommend to use the Unsöld recipe (Eqs. (E.5) and (E.1)) with an enhancement factor for the line width of 1.5, again based on Fig. F.2.

For all lines without broadening data the parameter value was set to zero in the line list. Finally, we note that collisional broadening does not significantly affect the equivalent widths $W$ of spectral lines with low ratios of $\log W / \lambda$. As a rough guideline, we can derive a limit of $\log W / \lambda \lesssim-4.8$ from Fig. 2 in Warner (1967), which corresponds to $W \lesssim 75-110 \mathrm{~m} \AA$ for the UVES580 wavelength range and $W \lesssim 130-145 \mathrm{~m} \AA$ for the wavelength range of the GIRAFFE HR21 setting. For such lines the value of the line broadening constant or the theory used should be less relevant.

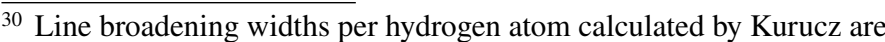
included in the line list in logarithmic form $\left(\log \gamma_{K}\right)$ for a temperature $T_{0}=10000 \mathrm{~K}$. These need to be scaled to the local temperature $T$ in the following way: $\gamma / N_{\mathrm{H}}=10^{\log \gamma_{K}}\left(T / T_{0}\right)^{0.3}$.
} 